$$
\begin{aligned}
& \text { UAM-I } \\
& \text { CBI }
\end{aligned}
$$

MODELADO MATEMATICO DE IA

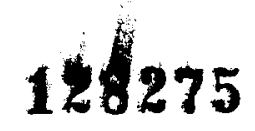

LIXIVIACION DE ARGENTITA CON

SULFATO FERRICO Y TIOUREA

TESIS QUE PRESENTA

GRETCHEN TERRI LAPIDUS LAVINE

PARA OBTENER EL GRADO DE

DOCTOR EN CIENCIAS

1993 
Esta tésis está dedicado a mi hijo Rodrigo quien tuvo que sufrir todo por "el bién de la ciencia".

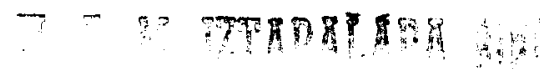


RESUMEN

Se plantea un modelo de difusión en una particula mineral para la lixiviación de argentita $\left(\mathrm{Ag}_{2} \mathrm{~S}\right)$ con tiourea $\mathrm{Y}$ sulfato férrico. De una manera novedosa, se considera la difusión de diecisiete especies, entre reactivos $y$ productos, que están en equilibrio simultáneo en solución. El modelo se basa en una conceptualización del núcleo decreciente en régimen transitorio con equilibrio en la frontera, para la reacción de oxidación o para un proceso de saturación en la superficie.

Para determinar la validez de la simulación numérica, se llevaron a cabo una serie de lixiviaciones sobre un concentrado mineral de sulfuros variando el porcentaje de sólidos y las concentraciones de tiourea, ácido sulfúrico y sulfato férrico en el intervalo de condiciones industriales. Los resultados experimentales en general concuerdan bien con las predicciones del modelo, permitiendo asi explicar algunos comportamientos no convencionales observados en este sistema complejo y analizando el paso controlante del proceso. Existe evidencia de la precipitación de un complejo sólido de plata en la frontera de reacción, que impide la extracción total de plata del mineral. Se encontró una expresión matemática que permite, por un lado, la predicción de la formación de éste $y$, por el otro, la saturación de la frontera de reacción y consecuentemente, el limite de la extracción total de plata, en determinadas condiciones de un mineral especifico. 


\section{INDICE}

CAPITULO I - INTRODUCCION

CAPITULO II - REVISION BIBLIOGRAFICA

II.1 - LIXIVIACION CON TIOUREA (3); II.2 - MODELO

TRADICIONAL DE NUCLEO DECRECIENTE Y SUS LIMITACIONES (13)

CAPITULO III - DESARROLLO TEORICO

CAPITULO IV - PROCEDIMIENTO EXPERIMENTAL IV.1 - REACTIVOS (24); IV.2 - EQUIPO (24); IV.3 PROCEDIMIENTO (26)

CAPITULO V - RESULTADOS Y DISCUSION

V.1 - RESULTADOS EXPERIMENTALES Y LOS AJUSTES DEL MODELO (28); V.2 - PREDICCIONES DEL MODELO PARA OTRAS CONDICIONES DE SOLUCION (42); V.3 - APLICABILIDAD A OTROS MINERALES (44)

CAPITULO VI - CONCLUSIONES

BIBLIOGRAFIA

NOMENCLATURA

APENDICE A - PROCEDIMIENTOS EXPERIMENTALES ESPECIFICOS A.1. - CONDICIONES EMPLEADAS PARA EL ANALISIS POR ABSORCION ATOMICA (60); A.2 - CUANTIFICACION DE TIOUREA TOTAL (60)

APENDICE B - ADIMENSIONALIZACION DE LAS ECUACIONES DEL MODELO

B.1. - BALANCE DE IAS ESPECIES EN EL SENO DE LA SOLUCION (BAL) (62); B.2. - JUSTIFICACION DE LA AGRUPACION DE ESPECIES (62); B.3. - ADIMENSIONALIZACION DE LA ECUACION DE DIFUSION (64)

APENDICE $C$ - PROGRAMA $Y$ CONSTANTES USADAS PARA RESOLVERLO C.1. - PROGRAMA (NO INCLUYE LOS SUBRUTINAS DE PAQUETE) (66); C. 2. - METODOS NUMERICOS UTILIZADOS EN EL PROGRAMA (87); C.3. - CONSTANTES DE EQUILIBRIO Y SU CORRECCION PARA LA FUERZA IONICA (89); C.4. - ESTIMACION DE LAS DIFUSIVIDADES LIBRES DE LAS ESPECIES (91); C.5. ESTIMACION DE LA DIFUSIVIDAD EFECTIVA Y LA POROSIDAD (93) i C.6. - EXTRACCION DE COBRE (95) 
EXPERIMENTO 0

EXPERIMENTO 00 (103)

EXPERIMENTO 2 (108)

EXPERIMENTO 4 (113)

EXPERIMENTO 5 (118)

EXPERIMENTO 6 (123)

EXPERIMENTO 7 (128)

EXPERIMENTO 8 (133)

EXPERIMENTO 9 (138)

EXPERIMENTO 10 (143)

EXPERIMENTO 11 (148)

EXPERIMENTO 12 (154)

EXPERIMENTO 13 (160)

EXPERIMENTO 14 (165)

EXPERIMENTO 15 (170)

EXPERIMENTO 17 (175)

EXPERIMENTO 18 (180)

EXPERIMENTO 19 (186) 


\section{INDICE DE FIGURAS}

Figura 1 - Modelo de Nucleo Decreciente en una Partícula . 18

Figura 2 - Sistema Experimental . . . . . . . . . . . . 25

Figura 3 - Experimento 9 (Modelo Original) . . . . . . . . 29

Figura 4 - Experimento 12 (Modelo Original) . . . . . . 30

Figura 5 - Experimento 18 (Modelo original) . . . . . . . 30

Figura 6 - Variación de Acido sulfúrico . . . . . . . . . . 36

Figura 7 - Variación de Sulfato Férrico . . . . . . . . . 36

Figura 8 - Variación de Tiourea (10 gramos de sólido) • • 37

Figura 9 - Variación de Tiourea (80 gramos de sólido) • . . 38

Figura 10 - Variación de Temperatura. . . . . . . . . . 38

Figura 11 - Variación de Porciento de Sólidos . . . . . . 39

Figura 12 - Perfiles de Concentración $\left(\mathrm{Fe}^{+3}\right.$ y $\left.\mathrm{AgT}_{2}^{+}\right)$. - . 39y40

Figura 13 - Lixiviación de Mineral (Simulación) . . . . . . 42

Figura 14 - Lixiviación con $\mathrm{H}_{2} \mathrm{O}_{2}$ (Efecto de Tiourea). . . 43

Figura 15 - Lixiviación con $\mathrm{H}_{2} \mathrm{O}_{2}$ (Efecto de \& sólidos). - . 43

Comparaciones entre simulaciones de los Modelos original $y$ Modificado para Cada Experimento

Experimento 0 . . . . . . . . . . . . . . . . . . 102

Experimento 00. . . . . . . . . . . . . . . . . . . 107

Experimento 2 . . . . . . . . . . . . . . . . . . . . 112

Experimento 4 . . . . . . . . . . . . . . . . . . . . . 117

Experimento 5 . . . . . . . . . . . . . . . . . . . 122

Experimento 6 . . . . . . . . . . . . . . . . . . . . . 127

Experimento 7 . . . . . . . . . . . . . . . . . . . . . 132

Experimento 8 . . . . . . . . . . . . . . . . . . . . . 137

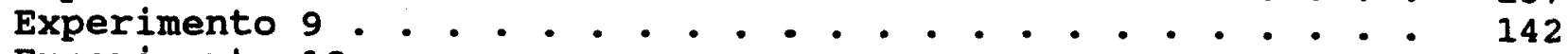

Experimento 10. . . . . . . . . . . . . . . . . . . . . 147

Experimento 11. . . . . . . . . . . . . . . . . . . . . . . 153

Experimento 12. . . . . . . . . . . . . . . . . . . . . . . 159

Experimento 13. . . . . . . . . . . . . . . . . . . . . . 164

Experimento 14. . . . . . . . . . . . . . . . . . . . . . . 169

Experimento 15. . . . . . . . . . . . . . . . . . . . . . . 174

Experimento 17. . . . . . . . . . . . . . . . . . . . . . . 179

Experimento 18. . . . . . . . . . . . . . . . . . . . . . . 185

Experimento 19. . . . . . . . . . . . . . . . . . . . . . . 191 
Introducción

CAPITULO I - INTRODUCCION

La lixiviación de metales preciosos con tiourea en medio ácido representa una alternativa menos tóxica y más selectiva que el proceso tradicional de cianuración. sin embargo, debido al costo elevado de la tiourea, la tioureación no es económicamente atractiva si no se reutilizan los reactivos, la tiourea y el ácido sulfúrico. Esto se lograría recirculando la solución lixiviante, después de haber recuperado los valores.

Para la optimización del proceso de lixiviación se requiere un conocimiento de la cinética de extracción bajo diferentes condiciones de solución, tales como niveles de concentración de sulfato férrico, de tiourea y de ácido, para diferentes contenidos de metales preciosos en el mineral. Sin embargo, se ha avanzado poco en esta dirección debido a la compleja interdependencia de los parámetros del sistema.

Un impedimento adicional para optimizar este sistema, $y$ en general en el campo de la lixiviación, es la ausencia de un modelo capaz de predecir la extracción de metales apartir de particulas minerales cuando existen consumo de reactivos $y / 0$ expresiones cinéticas complejas.

Por 10 anterior, se propone como objetivos de esta tesis: 1) implementar y comprobar la validez de un modelo matemático de tipo nucleo decreciente que describe la lixiviación de plata con sulfato férrico y tiourea en una particula mineral, considerando los principales equilibrios que se llevan a cabo en solución; y 2) determinar la influencia de diferentes parámetros de la solución sobre la lixiviación de dos de las más comunes estructuras mineralógicas: la plata metálica $\left(\mathrm{Ag}^{0}\right)$ y la argentita $\left(\mathrm{Ag}_{2} \mathrm{~S}\right)$. 


\section{Introducción}

A continuación se presenta el resultado del trabajo en el siguiente orden. En el Capítulo II (Revisión Bibliográfica) se plasma un resumen de la literatura concerniente, por un lado, con la lixiviación de metales preciosos con tiourea $y$, por el otro, con los modelos existentes de lixiviación. El desarrollo del modelo y el modelado de este sistema en particular, se presenta en el Capitulo III (Desarrollo Teórico). En el Capitulo IV (Desarrollo Experimental) se describe lo relacionado a la experimentación realizada para validar el modelo. La discusión de los resultados, tanto de los experimentos como de la predicciones númericas, se presentan en el Capitulo $\mathrm{V}$ (Resultados y Discusión). Finalmente, se ofrecen las implicaciones del presente trabajo en el capitulo VI (Conclusiones). 
Revisión Biblioaráfica

\section{CAPITULO II - REVISION BIBLIOGRAFICA}

\section{1 - LIXIVIACION CON TIOUREA}

El oro y la plata normalmente se encuentran en muy bajas concentraciones, finamente dispersos $y$ en su estado metálico nativo - de sulfuro dentro de minerales piriticos. El proceso de su lixiviación consiste en obtener iones metálicos disueltos en la disolución lixiviante; para lograr este fin se utiliza un agente oxidante el cual oxida los metales al estado iónico de valencia uno - bien oxida al sulfuro para transformarlo a azufre o sulfato, liberando de esta manera los iones de Ag(I). Posteriormente, se extraen los iones métalicos a la solución lixiviante mediante un agente complejante que solubilice preferentemente a éstos.

Desde hace cien años, se ha llevado a cabo en gran escala la lixiviación selectiva de oro y plata con un proceso conocido como la cianuración. Este proceso consiste de una oxidación-disolución de los metales usando aire $y$ cianuro de sodio en medio básico según las siguientes reacciones:

$$
\begin{aligned}
& 2 \mathrm{Au}^{\circ}(\mathrm{s})+4 \mathrm{CN}^{-}+\mathrm{O}_{2}+2 \mathrm{H}_{2} \mathrm{O} \longrightarrow 2 \mathrm{Au}(\mathrm{CN})_{2}^{-}(\mathrm{ac})+2 \mathrm{OH}^{-}+\mathrm{H}_{2} \mathrm{O}_{2} \\
& 2 \mathrm{Ag}^{\circ}(\mathrm{s})+4 \mathrm{CN}^{-}+\mathrm{O}_{2}+2 \mathrm{H}_{2} \mathrm{O} \longrightarrow 2 \mathrm{Ag}(\mathrm{CN})_{2}^{-}(\mathrm{ac})+2 \mathrm{OH}^{-}+\mathrm{H}_{2} \mathrm{O}_{2} \\
& 2 \mathrm{Ag}_{2} \mathrm{~S}(\mathrm{~s})+4 \mathrm{CN}^{-}+4 \mathrm{O}_{2}+4 \mathrm{H}_{2} \mathrm{O} \longrightarrow \mathrm{oben}
\end{aligned}
$$

El ión cianuro es muy eficiente para solubilizar el oro y la plata debido a su alto grado de complejación con estos metales (Smith y Martell, 1976). La solución lixiviante se mantiene a un pH alrededor de 10 con adiciones de cal o sosa para evitar la formación del ácido cianhídrico ( $\mathrm{HCN}$ ), sustancia altamente tóxica. La lixiviación se efectúa durante 24 a 72 horas a temperatura 
Revisión Bibliográfica

ambiente para mantener alta la solubilidad de oxígeno en la solución lixiviante.

A pesar de su extenso uso, el proceso de cianuración presenta varios problemas importantes. El primero concierne a la cinética tan lenta de la oxidación-disolución. Dadas las condiciones de operación requeridas para reducir la toxicidad del cianuro, no es posible utilizar agentes oxidantes fuertes, como iones metálicos, que incrementarian la cinética de oxidación. Aún más, por 10 anterior, la lixiviación no es completa para minerales refractarios y/O arcillosos, porque el oxígeno no es lo suficientemente agresivo como agente oxidante para atacar la estructura matricial que contiene al oro y a la plata encapsulados. Para liberar los metales se ha estudiado la posibilidad de moler mas finamente u oxidar el mineral con soluciones alcalinas antes de la cianuración. Sin embargo, en la mayoría de los casos, el incremento en el costo del proceso es prohibitivo (Royston et al, 1984).

El segundo problema es la baja selectividad del cianuro como agente complejante. Además de oro y plata, el cianuro se compleja fuertemente con otros iones producidos por la oxidación, como los del cobre, del arsénico, del hierro y del plomo, ocasionando un consumo excesivo de cianuro para lograr recuperaciones altas de los metales preciosos. Por esto, la lixiviación de oro y plata provenientes de minerales que han pasado por el proceso de flotación es menos rentable porque se concentran los metales cianicidos (Charley, 1983) y en aquellos minerales con alto contenido de arsénico $Y$ antimonio (Monhemius, 1987). Adicionalmente, el plomo contenido en el mineral forma un complejo sólido con el cianuro que pasiva la superficie del oro y de la plata durante la lixiviación (Habashi, 1970). 


\section{Revisión Bibliográfica}

El tercer problema es la elevada toxicidad de cianuro que ocasiona dificultades para su manejo $y$ desecho.

Por todo lo anterior, las investigaciones recientes sobre la lixiviación de oro y plata se han enfocado a la utilización de otros reactivos que eliminen los problemas ocasionados por el cianuro y que permitan el uso de agentes oxidantes más agresivos. Se ha encontrado que la sustancia complejante más prometedora es, en este sentido, la tiourea debido a su alto grado de complejación $y$ selectividad por el oro y la plata. Las reacciones de complejación de oro $y$ plata son las siguientes a una fuerza iónica constante e igual a uno (Pashkov et al, 1984 y Shivrin y Shivrina, 1976; Smith y Martell, 1982):

$$
\begin{aligned}
& \mathrm{Au}^{+}+2 \mathrm{CS}\left(\mathrm{NH}_{2}\right)_{2} \Longleftrightarrow \mathrm{Au}\left[\mathrm{CS}\left(\mathrm{NH}_{2}\right)_{2}\right]_{2}{ }^{+} \quad \log \mathrm{K}_{2}{ }^{\prime}=21.96 \\
& \mathrm{Ag}^{+}+\mathrm{CS}\left(\mathrm{NH}_{2}\right)_{2} \Longleftrightarrow \operatorname{AgCS}\left(\mathrm{NH}_{2}\right)_{2}^{+} \quad \log \mathrm{K}_{1}=7.11 \\
& \mathrm{Ag}^{+}+2 \mathrm{CS}\left(\mathrm{NH}_{2}\right)_{2} \Longleftrightarrow \mathrm{Ag}\left[\mathrm{CS}\left(\mathrm{NH}_{2}\right)_{2}\right]_{2}{ }^{+} \quad \log \mathrm{K}_{2}=10.61 \\
& \mathrm{Ag}^{+}+3 \mathrm{CS}\left(\mathrm{NH}_{2}\right)_{2} \Longleftrightarrow \mathrm{Ag}\left[\mathrm{CS}\left(\mathrm{NH}_{2}\right)_{2}\right]_{3}{ }^{+} \quad \log \mathrm{K}_{3}=12.73 \\
& \mathrm{Ag}^{+}+4 \mathrm{CS}\left(\mathrm{NH}_{2}\right)_{2} \Longleftrightarrow \mathrm{Ag}\left[\mathrm{CS}\left(\mathrm{NH}_{2}\right)_{2}\right]_{4}^{+} \quad \log \mathrm{K}_{4}=13.57
\end{aligned}
$$

El empleo de tiourea tiene varias ventajas sobre el uso de cianuro. En primer lugar, la tiourea es poco tóxica y no presenta problemas de manejo y/o desecho. Segundo, las lixiviaciones con tiourea se llevan a cabo en medio ácido, lo cual permite el uso de agentes oxidantes fuertes como los peróxidos $y$ el ión férrico. Además, el primer producto de oxidación de tiourea, disulfuro formamidina (DSFA), es un agente oxidante para la lixiviación (Preisler y Berger, 1947).

$2 \mathrm{CS}\left(\mathrm{NH}_{2}\right)_{2} \Longleftrightarrow\left(\mathrm{NH}_{2}\right) \mathrm{NHC}-\mathrm{S}-\mathrm{S}-\mathrm{CNH}(\mathrm{NH} 2)+2 \mathrm{H}^{+}+2 \mathrm{e}^{-} \mathrm{E}^{\circ}=0.42 \mathrm{~V}$ 
Revisión Bibliográfica

Finalmente, el consumo de tiourea por la complejación con los metales cianicidos es reducido, excepto en el caso de cobre (Smith y Martel1, 1982).

E1 uso de tiourea como agente complejante tiene una gran desventaja que ha impedido su comercialización: el alto grado de consumo debido a su degradación. El disulfuro formamidina (DSFA) y la tiourea se degradan según las siguientes reacciones (Bilston et al, 1984):

$$
\begin{gathered}
\left(\mathrm{NH}_{2}\right) \mathrm{NHC}-\mathrm{S}-\mathrm{S}-\mathrm{CNH}\left(\mathrm{NH}_{2}\right) \Longleftrightarrow \mathrm{CS}\left(\mathrm{NH}_{2}\right)_{2}+\mathrm{CNNH}_{2}+\underset{\text { Oxidación }}{\mathrm{S}^{\circ}} \\
\mathrm{CS}\left(\mathrm{NH}_{2}\right)_{2}+\mathrm{H}_{2} \mathrm{O}====\underset{\mathrm{O}}{\mathrm{H}_{2} \mathrm{~N}-\mathrm{C}-\mathrm{NH}_{2}+\mathrm{H}_{2} \mathrm{~S}} \text { Hidrólisis }
\end{gathered}
$$

Se ha encontrado que el consumo de tiourea por degradación aumenta notablemente con incrementos de acidez inferiores a $\mathrm{pH}=1.0$, temperaturas superiores a $40^{\circ} \mathrm{C}$, porcentaje de sólidos, concentración de tiourea y potencial de oxidoreducción de la solución lixiviante (Pyper y Hendrix, 1981a y b; Bilston et al, 1984 ; Nomvalo, 1986; Sandberg y Huiatt, 1986a y b). Se ha observado también que los productos de la oxidación irreversible de tiourea pueden pasivar la superficie de la reacción de oro $y$ formar complejos poco solubles con la plata (Groenewald, 1975, $1976 \mathrm{y}$ 1977; Ospanov et al, 1971; Chen et al, 1980; Goto et al, 1985; Pesic, 1988).

Uno de los avances más importantes para evitar la degradación de tiourea y, consecuentemente, mejorar la economía del proceso de acidotioureación fue hecho recientemente por Schulze (1984a), quien demostró que la adición de $\mathrm{SO}_{2}$ a la solución lixiviante puede reducir en un $95 \%$ el consumo de tiourea por oxidación irreversible debido a que este compuesto reduce selectivamente el disulfuro formamidina (DSFA). Además, como la presencia de $\mathrm{SO}_{2}$ mantiene reducida a la tiourea, se requiere menos de ésta última para 
Revisión Bibliográfica

complejar el oro y la plata (Schulze, 1984a; Deschenes y Ghali, 1988; Zegarra et al, 1989). Posteriormente, el mismo Schulze (1986) menciona que el $\mathrm{H}_{2} \mathrm{~S}$, el $\mathrm{ZnS}$, el $\mathrm{Na}_{2} \mathrm{~S}$ y el ion tiosulfato actúan de una manera similar a la del $\mathrm{SO}_{2}$. Sin embargo, Nomvalo (1986), utilizando un compuesto equivalente, el ión metabisulfito, llegó a la conclusión de que no hubo reducción en el consumo de tiourea. Posiblemente este comportamiento se debe a que el ión metabisulfito genera $\mathrm{SO}_{2}$ in situ, pero tarda demasiado para evitar la mayoria de la destrucción de la tiourea que se presenta en los primeros minutos de la lixiviación.

Los estudios cinéticos de la lixiviación de plata y oro en tiourea se han centrado en el uso de peróxido de hidrógeno, ión férrico y aire como agentes oxidantes. Para los agentes antes mencionados a condiciones óptimas de la solución, algunos autores reportan que la cinética para acidotioureación es un orden de magnitud mayor que la cinética de cianuración (Charley, 1983), aunque otros no han encontrado una mejora sustancial (Turner, 1986). Las reacciones de oxidación de oro por peróxido de hidrógeno, ión férrico y oxigeno, respectivamente, son (Hiskey, 1981):

$$
\begin{aligned}
2 \mathrm{Au}^{\circ}(\mathrm{s})+\mathrm{H}_{2} \mathrm{O} 2+2 \mathrm{H}^{+} & \Longleftrightarrow 2 \mathrm{Au}(\mathrm{I})+2 \mathrm{H}_{2} \mathrm{O} \\
\mathrm{Au}^{\circ}(\mathrm{s})+\mathrm{Fe}(\mathrm{III}) & \Longleftrightarrow \mathrm{Au}(\mathrm{I})+\mathrm{Fe}^{2+} \\
{ }^{2} \mathrm{Au}^{\circ}(\mathrm{s})+1 / 2 \mathrm{O}_{2}+2 \mathrm{H}^{+} & \Longleftrightarrow 2 \mathrm{Au}(\mathrm{I})+\mathrm{H}_{2} \mathrm{O}
\end{aligned}
$$

La mayoria de los investigadores han estudiado el efecto de los primeros dos agentes oxidantes, debido a la dificultad de aislar la cinética de oxidación por oxígeno en presencia de especies oxidadas de hierro, contenido en el mineral (Pyper $y$ Hendrix, 1981a y b y Gabra, 1984). 


\section{Revisión Bibliográfica}

Existen diferentes opiniones acerca de cuál es el agente activo en la oxidación de plata y oro. Groenewald (1976) menciona que, en los casos del peróxido de hidrógeno y oxígeno, estos dos agentes oxidan la tiourea a disulfuro formamidina (DSFA), según la reacción ( 8$)$, en el seno de la solución y el DSFA es el agente que oxida directamente a los metales. Sin embargo, para el ión férrico, debido al fenómeno de complejación múltiple, no se sabe cuál ó cuales de las especies son las responsables para la oxidación de los metales.

Las reacciones de los diferentes agentes oxidantes con la plata metálica son similares a las antes mencionadas con el oro. Sin embargo, cuando la plata se encuentra en forma sulfurada como argentita, el agente oxidante actúa sobre el sulfuro (Téllez et al., 1986 y contreras-Bustos, 1988). En este caso, estos autores proponen las siguientes reacciones de oxidación:

$$
\begin{aligned}
& \mathrm{Ag}_{2} \mathrm{~S}(\mathrm{~S})+\mathrm{H}_{2} \mathrm{O}_{2}+2 \mathrm{H}^{+} \Longleftrightarrow 2 \mathrm{Ag}(\mathrm{I})+2 \mathrm{H}_{2} \mathrm{O}+\mathrm{S}^{\circ} \\
& \mathrm{Ag}_{2} \mathrm{~S}(\mathrm{~S})+2 \mathrm{Fe}(\mathrm{III}) \quad \Longrightarrow 2 \mathrm{Ag}(\mathrm{I})+2 \mathrm{Fe}(\mathrm{II})+\mathrm{S}^{\circ} \\
& \mathrm{Ag}_{2} \mathrm{~S}(\mathrm{~S})+1 / 2 \mathrm{O}_{2}+2 \mathrm{H}^{+} \Longleftrightarrow 2 \mathrm{Ag}(\mathrm{I})+\mathrm{H}_{2} \mathrm{O}+\mathrm{S}^{\circ}
\end{aligned}
$$

Adicionalmente, existe una extracción no oxidativa por el ácido contenido en la solución (Contreras-Bustos, 1988)

$\mathrm{Ag}_{2} \mathrm{~S}(\mathrm{~S})+2 \mathrm{H}^{+} \Longleftrightarrow 2 \mathrm{Ag}(\mathrm{I})+\mathrm{H}_{2} \mathrm{~S}(\mathrm{~g})$

Aunque esta reacción sólo adquiere importancia en la ausencia de un agente oxidante.

Varios autores han estudiado los efectos de la concentración de agente oxidante, de tiourea y del pH, sobre la cinética de lixiviación de oro y plata. Todos han coincidido en que la cinética está controlada por un mecanismo difusivo de reactivos o productos, 
Revisión Bibliográfica

dependiendo de las condiciones de la solución lixiviante (Pyper y Hendrix, 1981a y b; Groenewald, 1975, 1976 y 1977; Hiskey, 1981; Lodeishchikov et al, 1975; Panchenkov et al, 1975 y 1976; Won Y Cho, 1985).

En cuanto al papel del agente oxidante, se ha encontrado que la cinética de extracción de ambos metales se incrementa linealmente con la concentración del agente oxidante o el potencial de oxidoreducción de la solución hasta un nivel, después del cual, la extracción se mantiene constante o disminuye (Bilston et al, 1984; Won Y Cho, 1985; Lodeishchikov et al, 1975; Turner, 1986; Groenewald, 1976; Songina et al, 1971). La disminución de la velocidad de extracción se produce por una falta de tiourea libre causada por su degradación oxidativa y/o por la complejación en el caso del ión férrico que forma la especie $\mathrm{FeSO}_{4}\left[\mathrm{CS}\left(\mathrm{NH}_{2}\right)_{2}\right]^{+}$ (Groenewald, 1975, 1976 y 1977; Lodeishchikov et al, 1975; Hiskey, 1983). Este último complejo formado en un medio de sulfato tiende a reducir el consumo de tiourea por oxidación, pero degenera en la especie reducida, $\mathrm{Fe}\left(\mathrm{SO}_{4}\right)\left[\mathrm{CS}\left(\mathrm{NH}_{2}\right)_{2}\right]$ (Hiskey, 1983 y Becker et al, 1983).

El nivel óptimo de agente oxidante para el oro ha sido determinado por varios autores a aquél que resulta en un potencial de oxidoreducción de entre 150 y $160 \mathrm{mV}$ referido al SCE (Groenewald, 1976; Pyper y Hendrix, 1981a y b; Bilston et al, 1984) aunque Nomvalo (1986) encontró el óptimo entre 200 y $250 \mathrm{mv}$. Groenewald (1975) encontró pasivación arriba de $255 \mathrm{mv}$ que se atribuyó a la formación de una película de sulfuro de oro por la descomposición de tiourea. Para la plata metálica, Lodeishchikov et al (1975) encontraron una velocidad máxima de disolución con una proporción de tiourea a DSFA de cuatro a uno, misma que corresponde a la relación estequiométrica de la siguiente reacción: 
Revisión Bibliográfica

$2 \mathrm{Ag}^{\circ}+\left(\mathrm{NH}_{2}\right) \mathrm{NHC}-\mathrm{S}-\mathrm{S}-\mathrm{CNH}(\mathrm{NH} 2)+2 \mathrm{H}^{+}+4 \mathrm{CS}\left(\mathrm{NH}_{2}\right)_{2} \Longleftrightarrow 2 \mathrm{Ag}\left[\mathrm{CS}\left(\mathrm{NH}_{2}\right)_{2}\right]_{3}^{+}$

Con respecto a la dependencia de la extracción con la concentración de tiourea en experimentos a concentración de ión férrico constante $y$ en exceso estequiométrico, Gabra et al (1984) y Groenewald (1976) encontraron que la cinética de la extracción de oro varía linealmente con la concentración de tiourea. Por otro lado, Pesic y Seal (1988 y 1990) observaron con un disco rotatorio de plata que la velocidad de extracción se incrementa con la concentración de tiourea hasta llegar a un máximo.

En todos los casos anteriores, cuando la concentración de tiourea es mayor que la del ión férrico, se observa una cinética mixta controlada por la difusión del agente oxidante como por el equilibrio con los productos en las soluciones de tiourea. Varios autores reportan energias de activación $\left(E_{a}\right)$ que son caracteristicas de procesos controlados por difusión; para oro Gabra observó una energía de $8.4 \mathrm{~kJ} / \mathrm{mol}$, Groenewald (1976) una de $28.0 \mathrm{~kJ} / \mathrm{mol}$ y Téllez et al (1986) una de $25.5 \mathrm{~kJ} / \mathrm{mol}$.

Para la extracción de plata, la energia de activación indica una cinética más compleja. Un cambio en el mecanismo controlante es evidente de los estudios de Lodeishchikov sobre plata metálica en disco rotatorio, ya que demuestra un aumento sustancial en $E_{a}$ conforme se reduce la concentración de tiourea (Lodeishchikov et al, 1975). Valores observados de la energia de activación del orden de $40.2 \mathrm{~kJ} / \mathrm{mol}$ a la más baja concentración de tiourea, pueden resultar de un control difusivo limitado por la solubilidad del producto o el arribo a equilibrio en el sitio de la reacción de oxidación (Lapidus y Mosqueira, 1988). A otras condiciones de solución, Téllez et al (1986) encontraron una $E_{a}=21.3 \mathrm{~kJ} / \mathrm{mol}$, un valor que indica un control difusivo de los reactivos. 
En varios estudios de lixiviación simultánea de oro y plata, se ha observado que la extracción de plata alcanza, a corto tiempo, un nivel máximo para luego disminuir alcanzando un valor estable con respecto al tiempo (Pyper y Hendrix, 1981a y b; Schulze, 1984a; Hiskey, 1983). Los autores han ofrecido dos explicaciones complementarias para éste fenómeno: que la plata precipita a consecuencia de la degradación de tiourea y que el incremento de la cantidad de oro extraido sustrae tiourea de la complejación con plata causando su precipitación. Independientemente de su causa, los autores coinciden en que se puede evitar la precipitación incrementando la concentración de tiourea. Por otro lado, Pachenkov et al (1975 y 1976) encontraron que la presencia de plata disminuye la velocidad de extracción del oro.

En cuanto al efecto del pH de la solución, se ha observado que, en sistemas que utilizan el peróxido de hidrógeno, la cinética de extracción de oro y plata incrementa rápidamente conforme se aumenta la acidez de la solución hasta un $\mathrm{pH}$ de uno, valor abajo del cual la degradación de tiourea es significativa (Bilston et al, 1984; Ospanov et al, 1971; Songina et al, 1971). Este resultado es lógico dado que la reducción del peróxido depende de la concentración del ión hidronio. Varios autores usando el ión férrico encontraron también un incremento en la extracción de oro conforme aumentaba la acidez aunque éste aumento fue menor al observado en los estudios usando peróxido (Pyper y Hendrix, 1981a y b; Gabra et al, 1984; Goto et al, 1985; Sandberg y Huiatt, 1986a y b). Los primeros atribuyeron este fenómeno a la concentración más alta de disulfuro formamidina (DSFA), que sirve de agente oxidante, a bajos valores del pH, mientras Gabra et al (1984) lo atribuyeron a una mayor solubilidad del ión auroso libre en solución. La observación de un incremento en el pH de la solución conforme procede la reacción parecería evidenciar la validez de la primera explicación dado que el ión hidronio se consume en la reducción del 


\section{Revisión Bibliográfica}

DSFA a la tiourea (Turner, 1986). En caso de la plata, won y Cho (1985) y Pesic y Seal (1988 y 1990), con plata metálica, y Sandberg y Huiatt (1986a y b), con cloruro de plata, no encontraron una influencia del nivel de $\mathrm{pH}$ sobre la extracción, mientras Téllez et al (1986), con argentita, notó un efecto importante similar a aquel observado para oro.

Los estudios del efecto de los metales cianicidos sobre la cinética de lixiviación de oro y plata han mostrado que el consumo de tiourea por complejación con los cianicidos es muy baja comparada con el consumo de cianuro en la cianuración (Charley, 1983; Bilston et al, 1984 ; Chen et al, 1980). Además, la tiourea se vuelve aún más selectiva para oro a altas concentraciones de ésta (Bilston et al, 1984). En cuanto al cobre, la adición de iones cúpricos incrementa la velocidad de extracción de oro y reduce la de plata, probablemente debido a la función dual que juega en la solución como agente oxidante $y$ acomplejante de tiourea $y$ a la diferencia que existe entre las constantes de estabilidad para ambos metales preciosos. No obstante lo anterior, se ha observado que solamente en el caso del plomo existe una fuerte dependencia entre el consumo de tiourea por complejación y la concentración del metal en el mineral que podria afectar de una manera importante la cinética de lixiviación de oro $y$ plata (Bilston et al, 1984; Hiskey, 1983; Tataru, 1968). Para evitar el consumo de tiourea por los metales cianicidos se ha sugerido su remoción mediante una lixiviación ácida previa a la tioureación ácida (Hiskey, 1981; Groenewald, 1976; Deschenes Y Ghali, 1988; Dupuis Y Ghali,1989).

Se ha comprobado que dos factores adicionales favorecen la cinética de lixiviación del oro en ciertos minerales. Lodeishchikov et al (1975) afirman que adiciones de policaprilamida a la solución lixiviante acelera la extracción de oro. En minerales con alto contenido de pirita se ha visto que una tostación previa a la 
Revisión Bibliográfica

tioureación ácida elimina la necesidad de adicionar ión férrico, de otras fuentes, a la solución lixiviante (Bodson, 1981 y Moussoulos et al, 1984).

De la revisión anterior se concluye que los fenómenos involucrados en tioureación ácida, como la complejación de los metales preciosos y cianicidos con la tiourea, la difusión y complejación del agente oxidante y la degradación de la tiourea, intervienen de manera simultánea en la determinación de la cinética de extracción de oro y plata. Por eso, se hace patente la necesidad de estudiar el proceso de tioureación ácida con miras hacia el desarrollo de un modelo mixto de control por difusión de los reactivos $y$ productos tomando en cuenta los fenómenos de complejación y el consumo de tiourea. Este modelo posteriormente será utilizado para evaluar y optimizar económicamente el proceso de lixiviación con tiourea. Cabe mencionar que dado que en la literatura no existe un estudio cinético sobre particulas minerales adecuado para validar un modelo, es necesario generar datos experimentales adecuados para este propósito.

II.2 - MODELO TRADICIONAL DE NUCLEO DECRECIENTE Y SUS LIMITACIONES

Se ha comprobado por microscopia electrónica que el movimiento del frente de reacción en una lixiviación oxidativa de un metal en particulas minerales no porosas se asemeja al encogimiento de un núcleo que contiene el metal sin reaccionar, dejando en su lugar el resto de material que es inerte a la acción del oxidante y los productos sólidos formados (Sohn, 1979). Por eso, muchos autores, entre los más recientes, Warren et al. (1985), Rath et al. (1988) y Fuerstenau et al. (1986), han aplicado, con éxito para ciertas condiciones, el modelo tradicional de núcleo decreciente, como fue presentado por Yagi y Kunii (1955), para describir la cinética de 


\section{Revisión Bibliográfica}

lixiviación de metales en particulas. Aunque la conceptualización de etapas en serie es básicamente correcta, las suposiciones tomadas para simplificar las matématicas restan generalidad a la solución. Como se verá a continuación, estas suposiciones imponen límites a la validez del modelo que impiden el uso para sistemas complejos como el de la lixiviación de plata con tiourea.

El modelo de núcleo decreciente parte de la Segunda Ley de Fick en coordenadas esféricas para describir la difusión del agente oxidante (A) a través de una capa de inertes (Levenspiel, 1972):

$$
\epsilon \frac{\partial C_{A}}{\partial t}=\frac{D_{A}}{R^{2}} \frac{\partial}{\partial R}\left[R^{2} \frac{\partial C_{A}}{\partial R}\right] \quad R_{C}<R<R_{p}
$$

donde, $\epsilon=$ Porosidad de la capa de inertes

$R=$ Posición radial

$R_{c}=$ Radio del núcleo decreciente

$R_{p}=$ Radio de la particula

$D_{A}=$ Difusividad del agente oxidante a través de los inertes

Se supone que el sistema se encuentra en el estado pseudoestacionario, esto es, que la concentración de agente oxidante no cambia con el tiempo:

$$
0=\frac{D_{A}}{R^{2}} \frac{\partial}{\partial R}\left[R^{2} \frac{\partial C_{A}}{\partial R}\right]
$$

Lo anterior implica que: 1) no se reduce la concentración de agente oxidante con el tiempo, es decir que se encuentra en gran exceso y 2) el movimiento de la frontera de reacción es más lenta que el establecimiento de perfil de concentración. La primera condición no se cumple en la mayoría de las operaciones industriales en donde se trabaja a una concentración cercana a la 
Revisión Bibliográfica

estequiométricamente necesaria para la reacción. La segunda condición solamente se cumple para minerales con una ley muy alta en donde la cantidad requerida de moles de agente oxidante para lixiviar el metal en un volumen de mineral supera por lo menos diez veces a la concentración de agente oxidante en solución (Taylor et al, 1983). Sólo se cumplen las dos condiciones cuando se lleva a cabo la lixiviación con un bajo porcentaje de sólidos y de no cumplirlas, tarda más tiempo de 10 predicho para la lixiviación (Taylor et al, 1983).

Las condiciones a la frontera para resolver analiticamente la ecuación de difusión son las siguientes:

en la frontera de reacción $\left(R_{c}\right), N_{A}=k_{s} c_{A}$

en la superficie de la particula $\left(R_{p}\right), N_{A}=k_{m n}\left(C_{A}{ }^{\circ}-C_{A}{ }^{R p}\right)$

donde $\mathrm{N}_{\mathrm{A}}$ = Densidad de flujo (flux) del agente oxidante

$\mathrm{k}_{\mathrm{S}} \mathrm{C}_{\mathrm{A}}=$ Tasa de reacción de primer orden

$k_{m A}=$ Coeficiente de transferencia de masa desde el seno de la solución hasta la superficie de la particula

Una limitación adicional al planteamiento anterior es que sólo considera la reacción de oxidación de primer orden irreversible con respecto a un solo agente oxidante. En el sistema de lixiviación con tiourea se requiere considerar por 10 menos dos agentes oxidantes intimamente ligados. Consecuentemente, el efecto de los otros reactivos, los productos o el porcentaje de sólidos no está reflejado en la expresión.

Para describir el movimiento del frente de reacción $y$ determinar la conversión, se lleva a cabo un balance de materia 
Revisión Bibliográfica

sobre la particula considerando la estequiometria de la reacción entre el agente oxidante y el metal a lixiviar (Levenspiel, 1972).

Resolviendo se obtiene el tiempo de lixiviación, $t$, para llegar a una conversión determinada, $x$.

$$
t=\frac{R_{p}^{2} \rho_{m}}{6 C_{A} D_{A}}\left[\frac{2 D_{A}}{R_{p} k_{m A}} X+1+2(1-X)-3(1-X)^{2 / 3}+\frac{6 D_{A}}{R_{p} k_{s}}\left(1-(1-X)^{1 / 3}\right]\right.
$$

donde $\rho_{m}=$ Densidad molar de la plata en el mineral

Para el caso en donde la difusión del agente oxidante a través de la capa de inertes constituye la etapa más lenta (controlante), la ecuación anterior se reduce a lo siguiente:

$$
t=\frac{R_{p}^{2} \rho_{m}}{6 C_{A} D_{A}}\left[1+2(1-X)-3(1-X)^{2 / 3}\right]
$$

De 10 anterior se concluye que las ecuaciones (21) y (22) sólo se aplican a un conjunto restringido de condiciones. Por esta razón, se justifica el desarrollo de un modelo capaz de simular la lixiviación de metales a partir de particulas minerales para sistemas y condiciones frecuentamente encontrados en la industria de la metalurgia extractiva, aunque la complejidad de las ecuaciones no permita una resolución analítica. Cabe aclarar que el modelo que se presenta a continuación todavia tiene muchas limitaciones en cuanto a la suposición de un solo tamaño de particula y que no considera la presencia de otras especies reaccionantes (metales) en el mineral. Sin embargo, constituye un avance con respecto al modelo tradicional de núcleo decreciente. 
Desarrollo Teórico

\section{CAPITULO III - DESARROLLO TEORICO}

Debido a la naturaleza poco porosa de las menas en donde se encuentran los minerales de plata, y a la rapidez de la reacción en comparación con el proceso difusional, se escogió un modelo de tipo núcleo decreciente, caracterizado por una reacción que ocurre únicamente en una frontera móvil. Las suposiciones adicionales que se hacen, y las cuales se justifican en un mineral de baja ley debido a la gran cantidad de inertes, son las siguientes: 1) el proceso se lleva a cabo isotérmicamente; y 2) la partícula es esférica $y$ mantiene su forma y tamaño a lo largo de la lixiviación. En el presente modelo se puede considerar que una de las siguientes reacciones de oxidación se encuentra en equilibrio (rápida $y$ reversible) en el frente de reacción, $R_{c}$ :

$$
\begin{aligned}
& \mathrm{Ag}^{\circ}(\mathrm{s})+\mathrm{Fe}^{3+}+2 \mathrm{CS}\left(\mathrm{NH}_{2}\right)_{2} \Longleftrightarrow \mathrm{Ag}\left[\mathrm{CS}\left(\mathrm{NH}_{2}\right)_{2}\right]_{2}^{+}+\mathrm{Fe}^{2+} \\
& \frac{1}{3} \mathrm{Ag} \mathrm{S}_{2} \mathrm{~S}(\mathrm{~s})+\mathrm{Fe}^{3+}+2 \mathrm{CS}\left(\mathrm{NH}_{2}\right)_{2} \Longleftrightarrow \mathrm{Ag}\left[\mathrm{CS}\left(\mathrm{NH}_{2}\right)_{2}\right]_{2}^{+}+\mathrm{Fe}^{2+}+\frac{1}{2} \mathrm{~S}^{\circ}\left(15^{\prime}\right)
\end{aligned}
$$

(La unica diferencia entre las dos constantes de equilibrio es el valor númerico). Adicionalmente, en todas partes de la solución existe un balance de carga. Las reacciones de complejación de plata con tiourea, ecuaciones (4) a (7), están en equilibrio, asi como las siguientes especies:

$$
\begin{aligned}
& \mathrm{Fe}^{3+}+\mathrm{SO}_{4}^{2-} \Longleftrightarrow \mathrm{FeSO}_{4}^{+} \\
& \mathrm{H}^{+}+\mathrm{SO}_{4}^{2-} \Longleftrightarrow \mathrm{HSO}_{4}^{-} \\
& \mathrm{Fe}^{2+}+\mathrm{SO}_{4}^{2-} \longrightarrow \mathrm{FeSO}_{4} \\
& \mathrm{Fe}^{3+}+\mathrm{SO}_{4}^{2-}+\mathrm{CS}\left(\mathrm{NH}_{2}\right)_{2} \Longleftrightarrow \mathrm{FeSO}_{4} \mathrm{CS}\left(\mathrm{NH}_{2}\right)_{2}^{+} \\
& 2 \mathrm{Fe}^{3+}+2 \mathrm{CS}\left(\mathrm{NH}_{2}\right)_{2} \Longleftrightarrow 2 \mathrm{Fe}^{2+}+2 \mathrm{H}^{+}+ \\
& \left(\mathrm{NH}_{2}\right) \mathrm{NHC}-\mathrm{S}-\mathrm{S}-\mathrm{CNH}\left(\mathrm{NH}_{2}\right)
\end{aligned}
$$

Para describir la difusión de cada una de las especies fluidas, tanto de reactivos como de productos a través de la capa 
de inertes, se emplea la segunda ley de Fick en coordenadas esféricas:

$$
\epsilon \frac{\partial C_{j}}{\partial t}=\frac{D_{j}}{R^{2}} \frac{\partial}{\partial R}\left[R^{2} \frac{\partial C_{j}}{\partial R}\right]+R_{f_{j}}-R_{d_{j}}
$$

$$
\text { donde, } \begin{aligned}
\mathbf{R}_{\mathrm{fj}} & =\text { velocidad de reacción de formación de } \mathbf{j} \\
\mathbf{R}_{\mathrm{dj}} & =\text { velocidad de reacción de desaparición de } \mathbf{j} \\
\epsilon & =\text { Porosidad de la capa de inertes }
\end{aligned}
$$

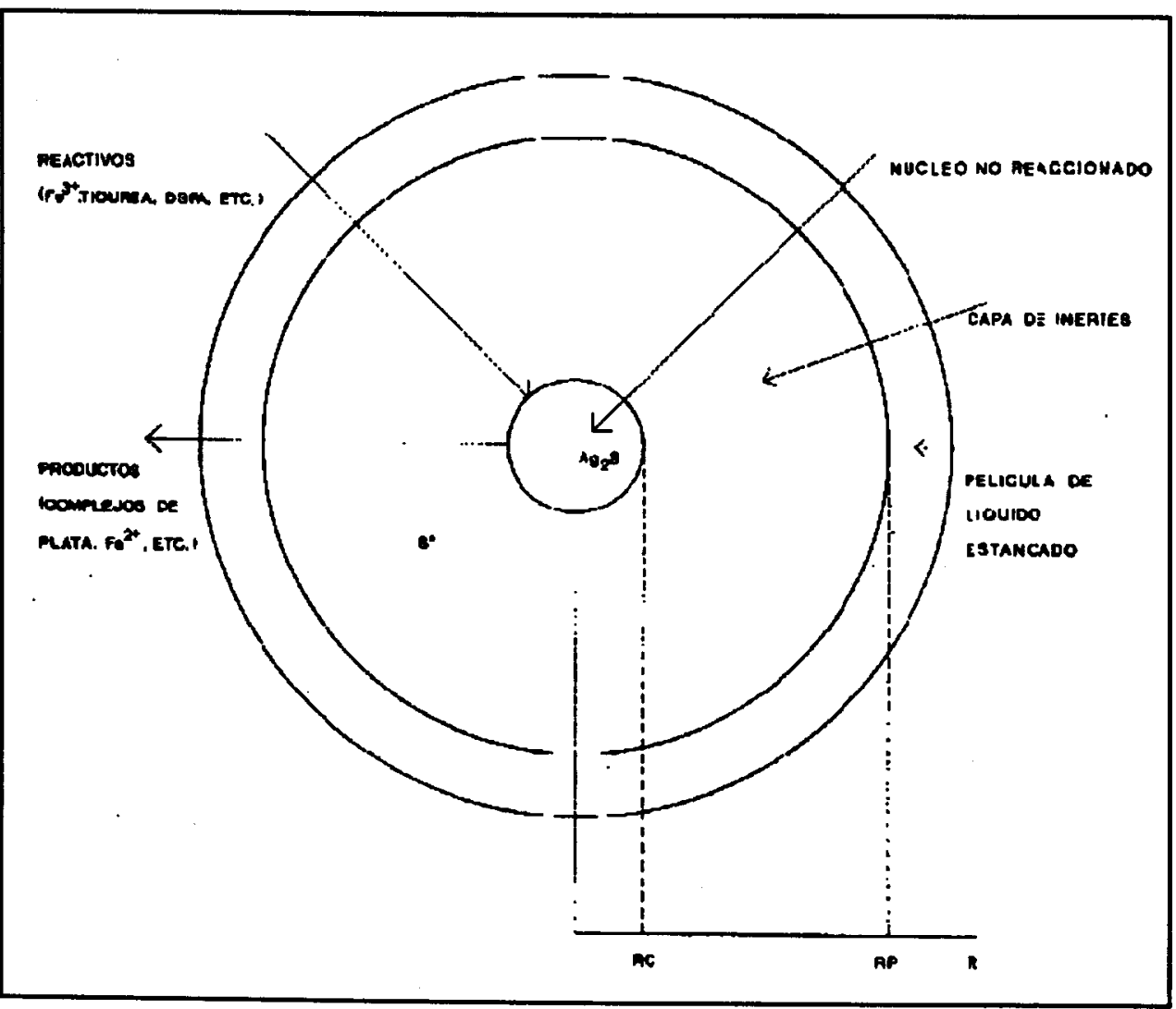

\section{FIGURA}

1 - MODELO DE NUCLEO DECRECIENTE EN UNA PARTICULA MINERAL

Cabe aclarar que $c_{j}$ es una concentración promedia tomada únicamente en el fluido. 
Desarrollo Teórico

Para facilitar el manejo de las ecuaciones se asigna a las especies la siguiente numeración:

$$
\begin{aligned}
& 1=\mathrm{Fe}^{3+} \\
& 2=\mathrm{SO}_{4}^{2-} \\
& 8=\mathrm{FeSO}_{4} \mathrm{CS}\left(\mathrm{NH}_{2}\right)_{2}^{+} \\
& 3=\mathrm{H}^{+} \\
& 9=\mathrm{HSO}_{4}^{-} \\
& 4=\mathrm{CS}\left(\mathrm{NH}_{2}\right)_{2} \\
& 10=\left(\mathrm{NH}_{2}\right) \mathrm{NHC}-\mathrm{S}-\mathrm{S}-\mathrm{CNH}\left(\mathrm{NH}_{2}\right) \\
& 5=\mathrm{Fe}^{2+} \\
& 11=\mathrm{FeSO}_{4} \\
& 6=\operatorname{Ag}\left[\mathrm{Cs}\left(\mathrm{NH}_{2}\right)_{2}\right]_{2}^{+} \\
& 12=\mathrm{Ag}^{+} \\
& 7=\mathrm{FeSO}_{4}^{+} \\
& 13=\mathrm{Ag}\left[\mathrm{CS}\left(\mathrm{NH}_{2}\right)_{2}\right]^{+} \\
& 14=\operatorname{Ag}\left[\mathrm{CS}\left(\mathrm{NH}_{2}\right)_{2}\right]_{3}{ }^{+} \\
& 15=\operatorname{Ag}\left[\mathrm{CS}\left(\mathrm{NH}_{2}\right)_{2}\right]_{4}^{+}
\end{aligned}
$$

Debido a que los equilibrios entre las diversas especies dependen del ambiente químico local en cada punto de la capa de inertes, para eliminar los términos de reacción de cada especie individual, se agrupan las especies por elemento o grupo funcional de la siguiente manera (ver el procedimiento detallado en el Apéndice B.3.):

$$
\begin{aligned}
& \text { Hierro Total }=\{\text { FT }\}=c_{1}+c_{5}+c_{7}+c_{8}+c_{11} \\
& \text { Sulfato Total }=\{\text { ST }\}=c_{2}+c_{7}+c_{8}+c_{9}+c_{11} \\
& \text { Tiourea Total }=\{\text { TT }\}=c_{4}+2 c_{6}+c_{8}+2 c_{10}+c_{13}+ \\
& 3 c_{14}+4 c_{15} \\
& \text { Agente oxidante Total }=\{\text { AT }\}=c_{1}+c_{7}+c_{8}+2 c_{10} \\
& \text { Plata Total }=\{\text { PT }\}=c_{6}+c_{12}+c_{13}+c_{14}+c_{15}
\end{aligned}
$$

Cabe mencionar que el balance de hidrógeno fue sustituido por balances de carga en toda la capa debido al error en que se podría incurrir al tener que considerar el agua y su equilibrio con el resto del sistema.

La ecuación de difusión (28) tomará la siguiente forma para los cinco especies totales: 


$$
\begin{gathered}
e \frac{\partial F T}{\partial t}=\frac{1}{R^{2}} \frac{\partial}{\partial R}\left[R^{2}\left(D_{1} \frac{\partial C_{1}}{\partial R}+D_{5} \frac{\partial C_{5}}{\partial R}+D_{7} \frac{\partial C_{7}}{\partial R}+D_{8} \frac{\partial C_{8}}{\partial R}+D_{11} \frac{\partial C_{11}}{\partial R}\right)\right] \\
e \frac{\partial S T}{\partial t}=\frac{1}{R^{2}} \frac{\partial}{\partial R}\left[R^{2}\left(D_{2} \frac{\partial C_{2}}{\partial R}+D_{7} \frac{\partial C_{7}}{\partial R}+D_{8} \frac{\partial C_{8}}{\partial R}+D_{10} \frac{\partial C_{10}}{\partial R}+D_{11} \frac{\partial C_{11}}{\partial R}\right)\right] \\
e \frac{\partial T T}{\partial t}=\frac{1}{R^{2}} \frac{\partial}{\partial R}\left[R^{2}\left(D_{4} \frac{\partial C_{4}}{\partial R}+2 D_{6} \frac{\partial C_{6}}{\partial R}+D_{8} \frac{\partial C_{8}}{\partial R}+2 D_{10} \frac{\partial C_{10}}{\partial R}+D_{13} \frac{\partial C_{13}}{\partial R}+3 D_{14} \frac{\partial C_{14}}{\partial R}+4 D_{15} \frac{\partial C_{15}}{\partial R}\right)\right] \\
e \frac{\partial A T}{\partial t}=\frac{1}{R^{2}} \frac{\partial}{\partial R}\left[R^{2}\left(D_{1} \frac{\partial C_{1}}{\partial R}+D_{7} \frac{\partial C_{7}}{\partial R}+D_{8} \frac{\partial C_{8}}{\partial R}+2 D_{10} \frac{\partial C_{10}}{\partial R}\right)\right] \\
\epsilon \frac{\partial P T}{\partial t}=\frac{1}{R^{2}} \frac{\partial}{\partial R}\left[R^{2}\left(D_{6} \frac{\partial C_{6}}{\partial R}+D_{12} \frac{\partial C_{12}}{\partial R}+D_{13} \frac{\partial C_{13}}{\partial R}+D_{14} \frac{\partial C_{14}}{\partial R}+D_{15} \frac{\partial C_{15}}{\partial R}\right)\right]
\end{gathered}
$$

Las condiciones iniciales para las cinco ecuaciones de difusión son las mismas concentraciones totales añadidas al sistema y la igualdad de los radios:

$$
\begin{aligned}
& a t=0,\{F T\}=\{F T\} \text { inicial }=\{F T\}^{0} \\
& \{\mathrm{ST}\}=\{\mathrm{ST}\}^{0} \\
& \{\mathrm{TT}\}=\{\mathrm{TT}\}^{0} \\
& \{\mathrm{AT}\}=\{\mathrm{AT}\}^{0} \\
& \{\mathrm{PT}\}=0 \\
& R_{c}=R_{p}
\end{aligned}
$$

A $t>0$, en $R=R_{p}$ (seno de la solución), las condiciones de frontera equivalen a balances de materia para cada especie total en solución. En el caso de hierro y sulfato totales, hay conservación 
de la suma de las especies a lo largo de la lixiviación. En cambio para la tiourea total, está definido con base en la cantidad aprovechable como acomplejante, es decir una vez degradada, no se considera como parte del sistema. Midiendo experimentalmente la velocidad de degradación de la tiourea, se ha observado que ésta obedece aproximadamente a una cinética de primer orden de acuerdo con las condiciones de cada experimento. Se utiliza el factor $\alpha$, evaluado para cada corrida experimental, para compensar la desaparición de la tiourea. Con respecto a la concentración total de agente oxidante en el seno de la solución (complejos del ión férrico y DSFA), se considera que se consume en la reacción de oxidación en la medida que se convierte la plata. Las condiciones de frontera descritas arriba son las siguientes:

$$
\begin{aligned}
& \{\mathrm{FT}\}=\{\mathrm{FT}\}^{0} \\
& \{\mathrm{ST}\}=\{\mathrm{ST}\}^{0} \\
& \{\mathrm{TT}\}=\{\mathrm{TT}\}^{0} \exp (-\alpha t) \\
& \{\mathrm{AT}\}=\{\mathrm{AT}\}^{0}-\{\mathrm{PT}\} \\
& \{\mathrm{PT}\}=\mathrm{Ba} * \mathrm{X} \quad \text { (Apéndice B.1.) }
\end{aligned}
$$

donde, $\mathrm{x}$ = Conversión fraccional

Bal = Acumulación de plata en la solución

$\alpha=$ Función de primer orden para la destrucción de tiourea a las condiciones de la solución (experimental)

$A t>0$, en $R=R_{c}$ (frontera de la reacción de oxidación), son la ecuación de equilibrio de la reacción de oxidación y las relaciones entre las densidades de flujo dictadas por la estequiometria de la misma reacción. 
Desarrollo Teórico

$$
K_{e q}=\frac{\left(C_{5}\right)\left(C_{6}\right)}{\left(C_{1}\right)\left(C_{4}\right)^{2}}=\frac{\left(F e^{2+}\right)\left(A g\left[C S\left(N H_{2}\right)_{2}\right]_{2}^{+}\right)}{\left(F e^{3+}\right)\left(C S\left(N H_{2}\right)_{2}\right)^{2}}
$$

$$
\begin{aligned}
& \text { Densidad de flujo de }\{\mathrm{FT}\}=0 \\
& \text { Densidad de flujo de }\{\mathrm{ST}\}=0 \\
& \text { Densidad de flujo de }\{\mathrm{TT}\}=0 \\
& \text { Densidad de flujo de }\{\mathrm{AT}\}= \\
& \text { - Densidad de flujo de }\{\mathrm{PT}\}
\end{aligned}
$$

donde, Densidad de flujo de $\{j T\}=\sum_{j} D_{j} \frac{\partial C_{j}}{\partial R}$

$j$ = especie que contiene el elemento o grupo funcional

La ecuación para el decrecimiento del núcleo $\left(R_{c}\right)$ con el tiempo es:

$$
\rho_{M} \frac{d R_{c}}{d t}=\text { Densidad de flujo de }\left.P T\right|_{R C}
$$

Después de adimensionalizar todas las ecuaciones (ver Apéndice B.2.), se someten las cinco ecuaciones diferenciales, sus respectivas condiciones de frontera y la de encogimiento del núcleo a una discretización mediante los métodos de colocación ortogonal $y$ de Euler implicito en las dimensiones radial $y$ temporal, respectivamente. El sistema de ecuaciones (compuesto por las diferenciales con sus condiciones iniciales y de frontera, la de encogimiento, las de equilibrio entre especies y las de balance de cargas) se resuelve por el método de Newton-Raphson para diferentes tiempos, empezando de un tiempo pequeño e integrando hasta el tiempo deseado. Una explicación más detallada de los métodos empleados se encuentra en el Apéndice C.2. La conversión fraccional 
a cada tiempo se calcula a partir de la posición del núcleo dado que:

$$
X=\frac{R_{c}^{3}}{R_{p}^{3}}
$$

Los valores numéricos de las constantes de complejación se encuentran reportados en la literatura y en el Apéndice C.3. Las constantes de equilibrio correspondientes a las reacciones de oxidación (12' y $15^{\prime}$ ), fueron calculadas a una fuerza iónica de uno a partir de los potenciales redox, las constantes de complejación $y$, en el caso de la argentita, el producto de solubilidad. Los valores obtenidos son los siguientes:

$$
\begin{aligned}
& \mathrm{K}_{\mathrm{eq}}=3.05 \times 10^{7} \quad \text { para la oxidación de } \mathrm{Ag}_{2} \mathrm{~S} \\
& \mathrm{~K}_{\mathrm{eq}}=1.9 .8 \times 10^{11} \quad \text { para la oxidación de } \mathrm{Ag}^{\mathrm{O}}
\end{aligned}
$$

Los valores de las difusividades libres fueron, en su mayoría, estimados por los métodos presentados en el Apéndice C.4. (Rubcumintara y Han, 1990; Pesic y Seal, 1990). 
Procedimiento Experimental

CAPITULO IV - PROCEDIMIENTO EXPERIMENTAL

IV. I - REACTIVOS

El mineral utilizado es un concentrado piritico proveniente de la mina de Real del Monte Y Pachuca, Hidalgo; tamizado en seco $Y$ en húmedo por las mallas 100, 150, 200,300, 325, 400 en tamices de acero inoxidable marca Mont-Inox $y$ usando un vibrador de tamices.

Se emplearon para este estudio dos tamaños de particula, $-300+325$ y $-325+400$ mallas, que corresponden a diámetros promedios de $47 \mu \mathrm{m}$ y $40 \mu \mathrm{m}$, respectivamente. La primera contiene en promedio $0.76 \%$ de plata, $3.5 \%$ de plomo, $1.9 \%$ de hierro y $0.3 \%$ de cobre; la segunda, $0.84 \%$ de plata, $5.1 \%$ de plomo, $2.3 \%$ de hierro y $0.3 \%$ de cobre. Las especies metálicas se encontraba en forma de sulfuros.

Todos los reactivos usados fueron de grado reactivo. Para los experimentos de lixiviación, se empleó agua destilada y para el análisis, agua tridestilada $y$ desionizada.

IV.2 - EQUIPO

Se llevaron a cabo las Iixiviaciones en un reactor de resinas de 1 litro de marca pyrex inmerso en un baño de temperatura controlada (Figura 2). La tapa del reactor tiene cuatro bocas: a través de la central, se instaló el sistema de agitación, el cual consistió de una varilla de vidrio con aspas de teflón $y$ un agitador marca Caframo modelo RZR-1; en uno de los orificios del contorno se introdujo la alimentación de nitrógeno (entrada $Y$ salida); en otro, se colocaron los electrodos de trabajo (platino) $y$ de referencia (sulfato de mercurio saturado) para medir el potencial de la solución antes $y$ durante la lixiviación con un 


\section{Procedimiento Experimental}

multimetro digital portátil marca BK Precision 2800; el orificio restante se utilizó para la toma de muestras a través de un tubo con fondo de vidrio poroso y una bomba de vacio. La medición del pH de la solución antes y después de la lixiviación se llevó a cabo mediante un potenciómetro marca conductronics modelo 20. Para controlar la temperatura del baño, se empleó, en la mayoría de las casos, un baño controlador-recirculador marca cole Parmer modelo $1268-00$.

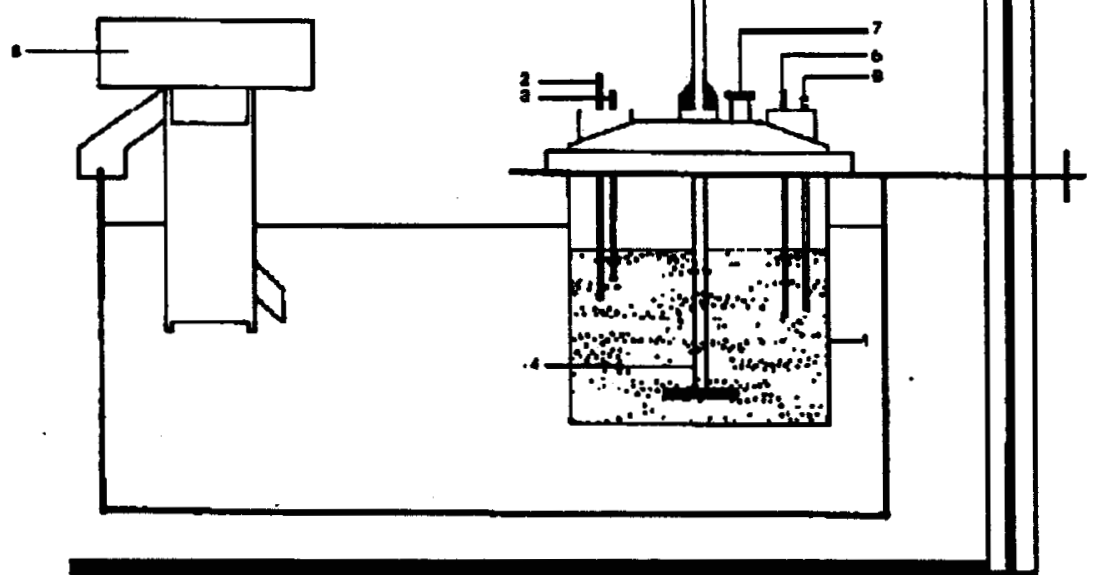

Figura 2 - Sistema experimental

1) Reactor de lixiviación

2) Electrodo de platino

3) Electrodo de referencia (sulfato de mercurio saturado)

4) Agitador de vidrio

5) Introducción de nitrógeno

6) Salida de nitrógeno

7) Puerta para la introducción de mineral, la toma de muestras liquidas y la medición del $\mathrm{pH}$.

8) Recirculador-Controlador de temperatura constante 
Procedimiento Experimental

El análisis de plata y cobre en solución se llevó a cabo utilizando un espectrofotómetro de absorción atómica marca varian modelo SpectrAA-20 con las condiciones especificadas en el Apéndice A.

IV. 3 - PROCEDIMIENTO

Se llevaron a cabo lixiviaciones del concentrado para las siguientes condiciones (los valores marcados entre corchetes se consideraron como base cuando cualquiera de los parámetros restantes fué variado):

\section{PARAMETRO}

Sulfato férrico, M Tiourea, $M$

Acido sulfúrico, $M$ Porciento de sólidos Temperatura, 'C

Velocidad de agitación, RPM

\section{VALORES EMPLEADOS}

$[0.003], 0.001,0.002$

$0.03,[0.10], 0.30$

$0.024,[0.041], 0.048,0.288$

$[1.3], 3.8,9.6$

$3,[21], 35$

[500]

Después de haber colocado $750 \mathrm{ml}$ de la solución en el reactor y añadido el ácido, se inició el burbujeo de nitrógeno y la agitación. Después de transcurridos aproximadamente 15 minutos, se tomó la lectura de potencial e inmediatamente después se añadió al sistema el mineral. Se tomaron muestras para análisis de $6 \mathrm{ml}$ y lecturas de potencial cada 15 minutos durante la primera hora, y posteriormente cada 30 minutos hasta completar los cinco horas del experimento. Adicionalmente, se tomaron muestras de $6 \mathrm{ml}$ para la cuantificación de tiourea total en los minutos 1,145 y 295. Estas últimas fueron puestas inmediatamente en contacto con polvo de zinc para evitar la descomposión posterior de la tiourea.

Cumplidos los 300 minutos, se paró la agitación y el flujo de nitrógeno, y se filtró la solución midiendo posteriormente su $\mathrm{pH}$. Después de haber secado el residuo, se tomaron muestras de éste 


\section{Procedimiento Experimental}

para su digestión en acido nitrico $y$ agua regia, para la cuantificación de plata y cobre, la primera, y de oro, la segunda. No se consideraron válidos los resultados de los experimentos en donde no cerraban los balances metalúrgicos de plata y de cobre.

Cabe mencionar algo acerca del extremo cuidado en cuanto al manejo de muestras se refiere. Como existe en este sistema la posibilidad de precipitación de los productos por limitaciones de solubilidad, es importante mantener las muestras, asi como el material de vidrio que entra en contacto con éstas, a una temperatura igual o ligeramente superior que la del experimento, hasta poder diluirlas. Además, para evitar la precipitación de plata debido la degradación con el tiempo de la tiourea, es aconsejable analizar las muestras 10 más pronto posible después de haberse terminado el experimento. 
Resultados y Discusión

CAPITULO V - RESULTADOS Y DISCUSION

\section{V.1 - RESULTADOS EXPERIMENTALES Y LOS AJUSTES DEL MODELO}

Como los resultados experimentales y los ajustes e interpretaciones derivados del modelo están intimamente relacionados, se organiza esta sección de la siguiente manera: en primer lugar, se presentan los resultados y predicciones del modelo original, como se desarrolla en el capitulo III, el cual no considera precipitación de plata. Finalmente, dado que existe evidencia de precipitación a ciertas condiciones de la solución, se discuten los fenómenos adicionales que se tiene que considerar en el modelo original para poder predecir los datos experimentales.

Cabe justificar, antes de empezar a discutir los resultados experimentales, los intervalos seleccionados de las variables de solución para probar el modelo de lixiviación. Como se ha visto anteriormente en el capitulo II (Revisión Bibliográfica), la lixiviación tanto de plata metálica como de argentita demuestra comportamientos anómalos (máximos) con respecto a la variación en las concentraciones de tiourea, sulfato férrico e ión hidronio. Los diferentes autores atribuyen estos comportamientos a distintas causas, en la mayoría de los casos a la destrucción de la tiourea y la compleja química del sistema. Sin embargo, no ha habido un estudio anterior en donde se combine el uso de un modelo que incorpora los múltiples equilibrios y procesos difusionales junto con datos experimentales provenientes de un sistema de particulas bien caracterizadas. Precisamente, se fijaron las concentraciones de estudio por ser aquéllas alrededor de las cuales los otros autores han observado las anomalias: 0.03-0.30M de tiourea, 0.003$0.01 \mathrm{M}$ de sulfato férrico y $0.024-0.288 \mathrm{M}$ de ácido sulfúrico. Además, son las condiciones en donde sería económicamente viable operar una 
lixiviación de concentrados con caracteristicas similares a las empleadas en el presente trabajo.

Con respecto al intervalo para estudiar el efecto del porcentaje de sólidos, el valor menor de $1.3 \%$ corresponde a una cantidad de plata en el sistema que cumple con las condiciones de pseudo-estadoestacionariadad del modelo tradicional, 0 sea $C_{A} / \Gamma_{M}<$ 0.1 y $C_{A}$ en exceso (Taylor et al., 1983). El concentrado mineral fué cuidadosamente seleccionado por su alto contenido de plata para que, a las concentraciones industriales mencionadas arriba, cumpliera con los condiciones antériores. El valor alto de porciento de sólidos, $9.6 \%$, es aproximadamente $20 \%$ menos de la cantidad de plata necesaria para consumir todo el ión férrico en este sistema. Considerando la pequeña cantidad de otras especies reaccionantes, como cobre, se estimó que no se llegaría al agotamiento del agente oxidante, aún a este alto porcentaje de sólidos.

EXTRACCION DE PLATA

Modelo Original

Los resultados de dos experimentos representativos $y$ sus respectivas predicciones con el modelo original se muestran en las Figuras 3 y 4 , como la conversión de plata $(X)$ contra el tiempo. Se observa que mientras la simulación para el experimento 9 a $0.1 \mathrm{M}$ tiourea reproduce adecuadamente la tendencia $y$ los valores

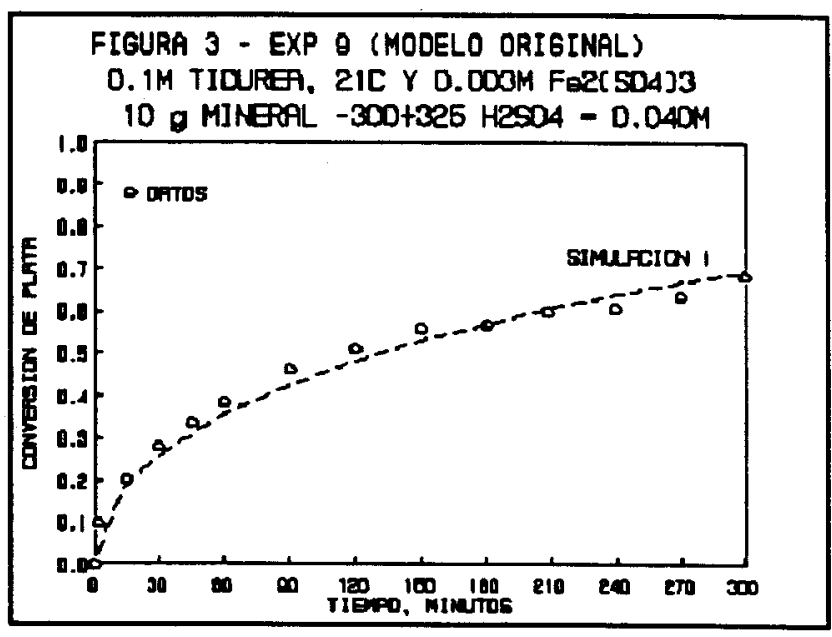
numéricos, la correspondiente para el experimento 12 a $0.3 \mathrm{M}$ tiourea 
Resultados y Discusión

no es tan afortunada (Figura 4). En el experimento 12, la conversión experimental tiende a un valor asintótico, tal vez por la saturación por la plata de la solución. Esta característica se repite en todos los experimentos a $0.3 \mathrm{M}$ de tiourea. A una concentración mayor de sulfato férrico, se puede notar que a altas conversiones de plata la desviación entre la conversión predicha y la experimental es apreciable (Figura 5 ).
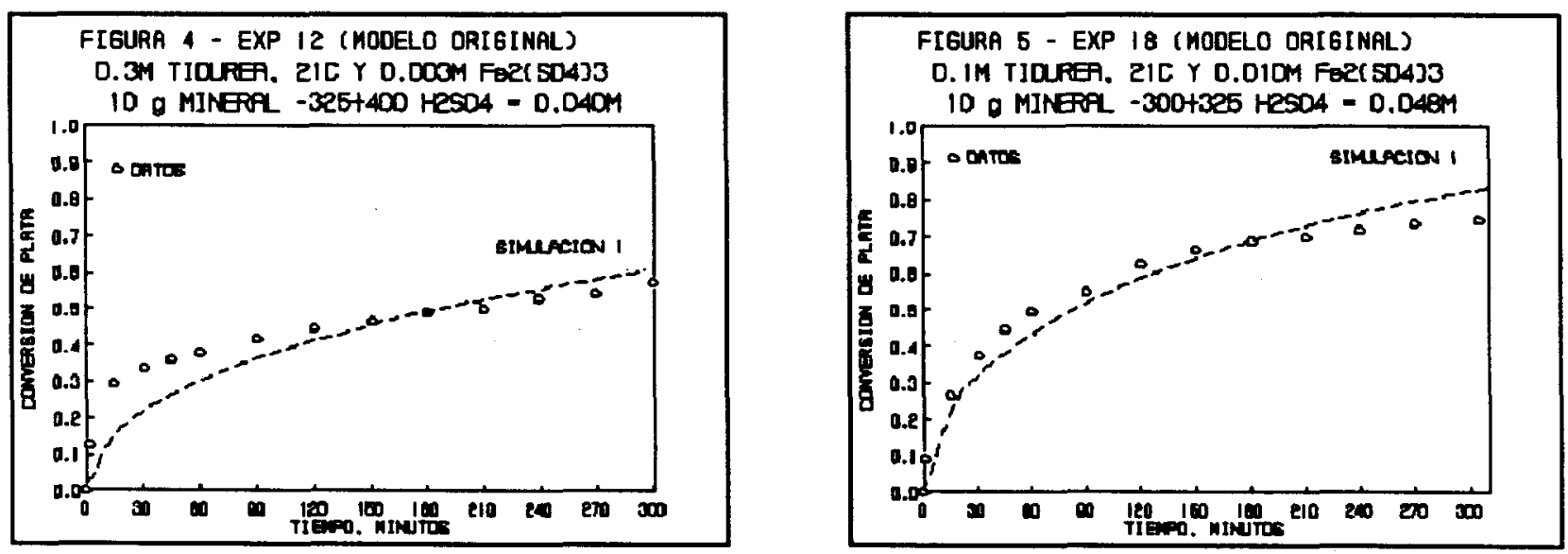

Para ajustar el valor de la difusividad efectiva $\left(D_{\mathrm{Fe}}\right)$, se emplea un solo juego de datos de conversión y tiempo para cada experimento (ver Apéndice C.2). A la misma temperatura las $D_{\mathrm{Fe}}$ deberian de coincidir aproximadamente, considerando que la fuerza iónica de las diferentes soluciones son parecidas. En la Tabla I, donde aparecen las difusividades efectivas para cada experimento a $21^{\circ} \mathrm{C}$, se observan dos comportamientos anómalos:

1) Los valores de $D_{\mathrm{Fe}}$, para los experimentos usando mayores cantidades de sulfato férrico, son bajos con respecto a los de las condiciones estándares, $0.006 \mathrm{M} \mathrm{Fe}^{3+}\left[00.003 \mathrm{M} \mathrm{Fe} \mathrm{F}_{2}\left(\mathrm{SO}_{4}\right)_{3}\right]$ y $0.1 \mathrm{M} \operatorname{cs}\left(\mathrm{NH}_{2}\right)_{2}$.

2) En la medida que aumenta la cantidad de mineral, a las mismas condiciones donde el valor de $\mathrm{D}_{\mathrm{Fe}}$ debería de ser igual, se ajusta una $\mathrm{D}_{\mathrm{Fe}}$ menor para compensar por la velocidad de 
Resultados y Discusión

lixiviación también menor (ver los grupos de experimentos 6-78 y 9-10-11 en la Tabla I).

TABLA I - DIFUSIVIDADES EFECTIVAS AJUSTADAS PARA LOS EXPERIMENTOS A $21^{\circ} \mathrm{C}$ (CONDICIONES ESTANDARES: $0.006 \mathrm{M} \mathrm{Fe} t^{3+}$ Y $0.1 \mathrm{M}$ $\mathrm{CS}\left(\mathrm{NH}_{2}\right)_{2}$

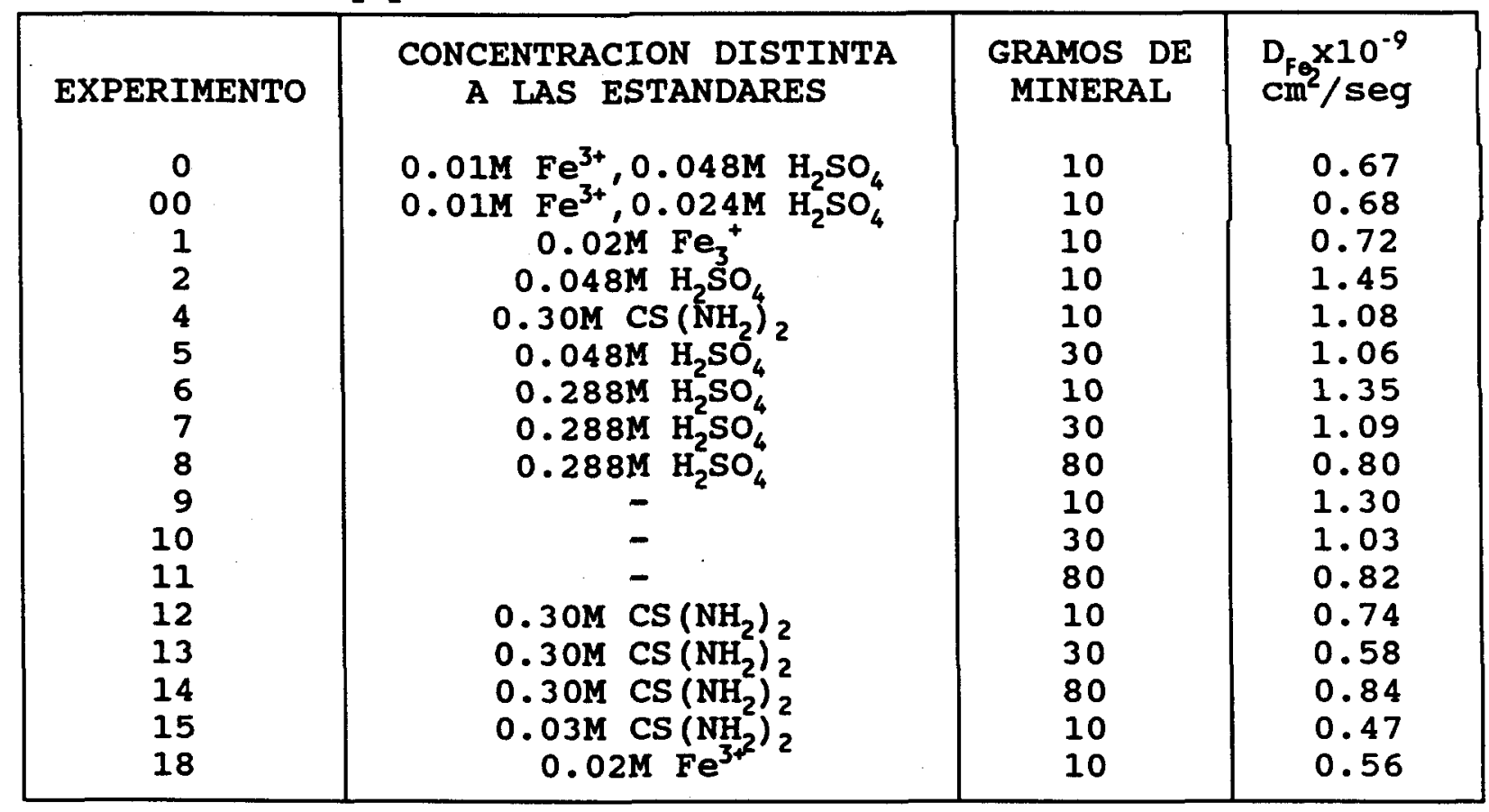

La evidencia anterior apunta hacia un sistema en donde existe un límite de solubilidad de alguno de los productos de la plata. Si éste es el caso, dado que las concentraciones de plata son más altas cerca de la frontera de reacción $\left(R_{c}\right)$, se esperaria que el precipitado se presentara primero en este sitio. Pesic y seal (1990) notaron la presencia de un precipitado cristalino sobre el disco rotatorio de plata en todos los experimentos de este sistema [0.001-0.050M $\mathrm{Fe}^{3+}$ y $\left.0.01-0.50 \mathrm{M} \mathrm{CS}\left(\mathrm{NH}_{2}\right)_{2}\right]$. Ellos, mediante varios métodos analiticos, identificaron el precipitado como $\mathrm{Ag}_{2}\left[\mathrm{CS}\left(\mathrm{NH}_{2}\right)_{2}\right]_{3} \mathrm{SO}_{4} \cdot \mathrm{H}_{2} \mathrm{O}$. Es interesante notar en su trabajo (Pesic $\mathrm{Y}$ Seal, 1990) que la velocidad de lixiviación de plata no es 
Resultados y Discusión

constante con el tiempo para concentraciones altas de tiourea, 10 cual sugiere pasivación de la superficie o saturación de la solución.

Una evidencia adicional para apoyar la teoria de que la precipitación de algún compuesto de plata interviene en el proceso, es el valor de la energía de activación. Con base en las $D_{\mathrm{Fe}}$ ajustadas en el modelo original y que aparecen en la Tabla II, la energia de activación es $43.1 \mathrm{~kJ} / \mathrm{mol}$, valor demasiado alto para un proceso de lixiviación controlado por difusión (Sohn, 1979; Lapidus y Mosqueira, 1988). Lodeishchikov et al (1975) también encontraron valores altos para la energia de activación, $40.2 \mathrm{~kJ} / \mathrm{mol}$ a $0.065 \mathrm{M}$ tiourea, en un disco rotatorio de plata metálica.

TABLA II - DIFUSIVIDADES EFECTIVAS AJUSTADAS PARA TRES TEMPERATURAS

(CONDICIONES: $0.1 \mathrm{M} \mathrm{CS}\left(\mathrm{NH}_{2}\right)_{2}, 0.006 \mathrm{M} \mathrm{Fe}^{3+}$ y $0.04 \mathrm{OM} \mathrm{H}_{2} \mathrm{SO}_{4}$ )

\begin{tabular}{|c|c|c|c|}
\hline EXPERIMENTO & TEMPERATURA, ${ }^{\circ} \mathrm{C}$ & $\begin{array}{c}\text { GRAMOS DE } \\
\text { MINERAL }\end{array}$ & $\begin{array}{c}\mathrm{D}_{\mathrm{F}_{2}} \times 10^{-9} \\
\mathrm{Cm}^{2} / \mathrm{seg}\end{array}$ \\
17 & 35 & 10 & 2.54 \\
9 & 21 & 10 & 1.30 \\
19 & 3 & 10 & 0.36 \\
\hline
\end{tabular}

Entonces, se puede inferir de 10 anterior que la extracción de plata, por lo menos en ciertos condiciones, puede estar controlada por difusión ó por solubilidad de los productos $y$ no por la difusión de los reactivos, como se plantea en el modelo original. La verdadera importancia de esta diferencia no estriba en lograr buenas predicciones de los datos experimentales, sino bajo qué condiciones es posible evitar el acercamiento a la saturación de la solución. Cuando este último fenómeno ocurre, disminuye la velocidad de lixiviación. Un modelo que no es capaz de predecir los límites de la lixiviación es poco útil en la práctica. 
Resultados y Discusión

Para lograr un modelado correcto es necesario modificar el modelo original para que, en primer lugar, verifique si existe precipitación en la superficie $y$, en caso de que exista, sustituir una condición de frontera en $R_{c}$ para considerar la disolución del precipitado, o sea sustituir la ecuación (45) por un producto de solubilidad del precipitado de plata.

En la literatura se reporta la existencia de dos productos sólidos: $\mathrm{Ag}_{2}\left[\mathrm{CS}\left(\mathrm{NH}_{2}\right)_{2}\right]_{3} \mathrm{SO}_{4}$ (p1) y $\mathrm{Ag}_{2}\left[\mathrm{CS}\left(\mathrm{NH}_{2}\right)_{2}\right]_{6} \mathrm{SO}_{4}$ (p2) (Pesic y Seal, 1990; Khmel'nitskaya et al, 1985). El primer problema con que se enfrenta, es que no existe explicitamente en la literatura ni productos de solubilidad $\mathrm{ni}$ constantes de formación para esos dos compuestos. Los segundos autores solamente reportan las solubilidades en agua acidificada de ambos sólidos: $5.6 \times 10^{-6} \mathrm{M}$ para el p1 y $8.8 \times 10^{-5} \mathrm{M}$ para el p2 a $25^{\circ} \mathrm{C}$. También mencionan que "cuando se eleva la temperatura a $50^{\circ} \mathrm{C}$, la solubilidad de $\mathrm{Ag}_{2}\left[\mathrm{CS}\left(\mathrm{NH}_{2}\right)_{2}\right]_{3} \mathrm{SO}_{4}$ se incrementa tres veces y la de $\mathrm{Ag}_{2}\left[\mathrm{CS}\left(\mathrm{NH}_{2}\right)_{2}\right]_{6} \mathrm{SO}_{4}$, seis veces". Estos valores corresponden a energías de activación de $35.1 \mathrm{~kJ} / \mathrm{mol}$ para el p1 y $57.3 \mathrm{~kJ} / \mathrm{mol}$ para el $\mathrm{p} 2$.

Para considerar la formación de cualquiera de los dos sólidos, se tiene que incluir en el modelo sus respectivos precursores solubles dentro del término de plata total, \{PT\}. La manera detallada de estimar las constantes de formación de los complejos solubles se encuentra en el Apéndice c. 3 .

Aunque Pesic y Seal (1990) determinaron que el sólido que se formaba en la superficie del disco rotatorio de plata es el compuesto pl, no es totalmente consistente con los resultados obtenidos para particulas minerales en el presente estudio. Por ejemplo, Khmel'nitskaya et al (1985) encontraron que la solubilidad de $\mathrm{pl}$ se reduce con un incremento en la concentración de ácido, mientras la de p2 no. Básicamente en el presente estudio tampoco se 
Resultados y Discusión

observó un efecto de la concentración del ácido sulfúrico. Además, la energia de activación calculada aqui es más cercana al valor aproximado para el p2 a partir de los datos de solubilidad. Lo anterior, aunado a lo expuesto en el Apéndice C.3, hace pensar que el compuesto que se forma es el p2.

Sin embargo, lo anterior no explica la dependencia nula con respecto al ión sulfato. Cualquier producto de solubilidad debería de incluir una forma del anión. Aquí se retoma el mecanismo planteado por Pesic y Seal (1990), donde el ión FesO ${ }_{4}\left[\mathrm{CS}\left(\mathrm{NH}_{2}\right)_{2}\right]^{+}$ funge de intermediario para la formación del producto sólido, ya que este se mantiene relativamente constante. Un hecho que apoya este mecanismo, $y$ que se verá mas adelante en la sección $V .2$, es que en ausencia de sulfato férrico (usando el $\mathrm{H}_{2} \mathrm{O}_{2}$ como oxidante), no se forma el producto sobre la superficie del disco de plata (Pesic y Seal, 1990), y se lixivia la plata más rápidamente.

Aunque de ninguna manera el mecanismo planteado por Pesic $y$ Seal (1990) sea correcto porque la reacción no está balanceada, este mecanismo explica de alguna manera la presencia de un estado de saturación en la interfase, que depende del siguiente producto de concentraciones:

$$
\mathrm{K}=\left[\mathrm{Ag}_{2}\left[\mathrm{CS}\left(\mathrm{NH}_{2}\right)_{2}\right]_{6}{ }^{2+}\right]\left[\mathrm{FeSO}_{4}\left[\mathrm{CS}\left(\mathrm{NH}_{2}\right)_{2}\right]^{+}\right]
$$

El valor del $\mathrm{K}$ empleado es de $1.5 \times 10^{-10}$ a $25^{\circ} \mathrm{C}$ porque produce $1 \mathrm{a}$ mejor predicción para todos los experimentos. Se usa una entalpia (energia de activación) de $44.0 \mathrm{~kJ} / \mathrm{mol}$ por la misma razón.

El ajuste de las constantes no resulta trivial porque como el fenómeno controlante del proceso depende directamente de los valores numéricos asignados a las constantes, es muy importante que estos sean precisos. Además, es necesario que las cantidades 
calculadas, como los perfiles de concentración y las $\mathrm{D}_{\mathrm{Fe}}$, sean consistentes con lo observado experimentalmente. Por ejemplo, se consiguen predicciones mucho mejores usando un $\mathrm{K}$ que sólo depende del catión $\left[\mathrm{Ag}_{2}\left[\mathrm{CS}\left(\mathrm{NH}_{2}\right)_{2}\right]_{6}{ }^{2+}\right]$, pero este planteamiento presenta dos desventajas: 1 ) se requiere un $K$ distinto para cada cantidad de sólidos y 2) predice precipitación cuando se usa peróxido de hidrógeno. Considerando la naturaleza aproximada de todas las constantes utilizadas en el presente estudio, las predicciones resultaron bastante cercanas al comportamiento experimental $y$, en el caso de las condiciones en donde los resultados de la simulación con el modelo original no son buenos, no se obtienen predicciones peores (Ver Apéndice $D$ ).

Modelo Modificado

Los resultados experimentales y sus respectivas simulaciones se presentan en esta sección como conversión fraccional de plata (X) contra tiempo, agrupados según la variable que cambia en cada caso: concentraciones de ácido sulfúrico, de sulfato férrico y de tiourea. Posteriormente, se muestra el mismo comportamiento con respecto a la temperatura y al porcentaje de sólidos. Finalmente, después de comentar los perfiles de cada especie, se discutirán algunas implicaciones en cuanto a la optimización de este sistema en particular.

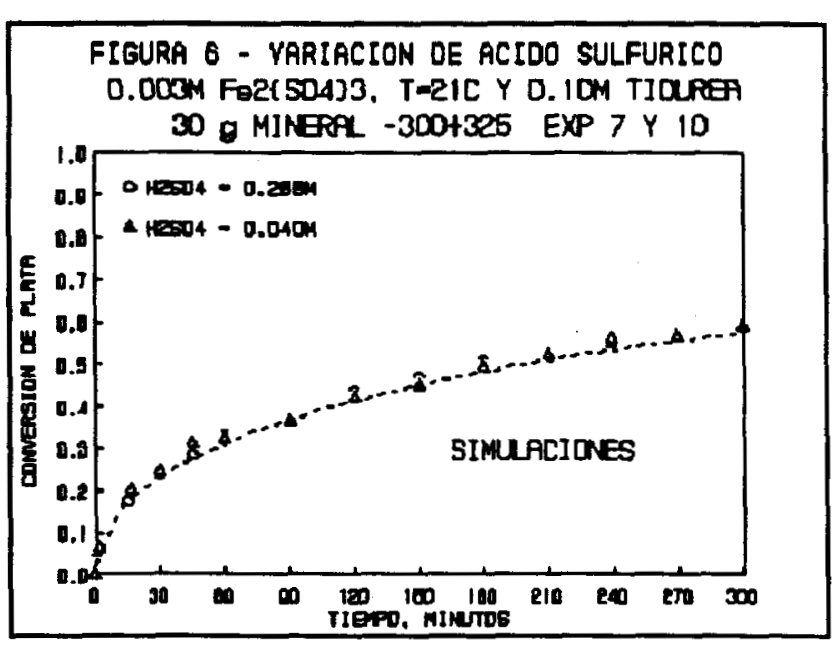

En la Figura 6 se presentan los resultados para dos experimentos con diferentes concentraciones de ácido sulfúrico. Se 
Resultados y Discusión

observa que no existe un efecto aparente de la concentración de ácido sulfúrico a $0.1 \mathrm{M}$ de tiourea, en concordancia con 10 que vió Pesic y seal (1990). Este comportamiento se debe a que a esta concentración puede haber precipitación del sólido $p_{2}$.

El efecto del sulfato férrico es más complicado porque actúa directamente sobre el producto $\mathrm{K}$. Se observa en la Figura 7 que, contrario a lo esperado para sistemas controlados por difusión de reactivos únicamente, la velocidad inicial de

lixiviación para la

FIGURA 7 - VARIACION DE SULFATO FERRICO

$0.1 M$ TIOUREA, T-ZIC Y EXP 0,18 Y 9

10 ด MINERAL $-300+325$ H2SO4 $=0.048 M$

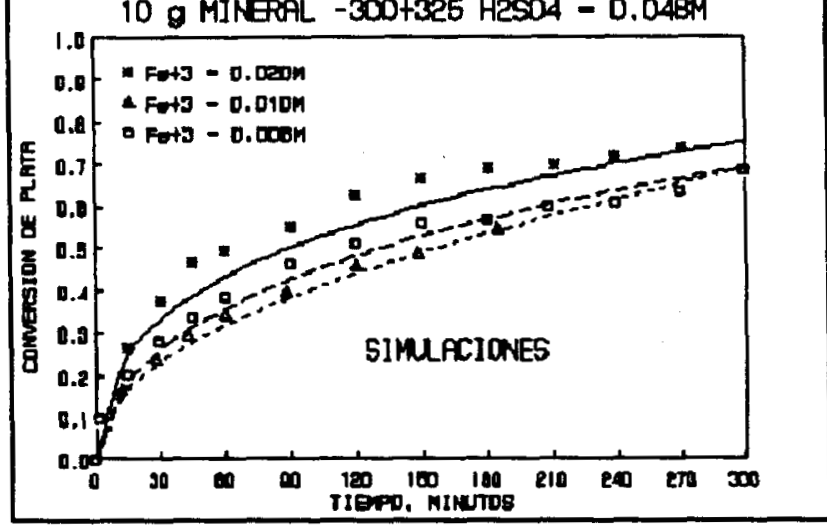

concentración menor de sulfato férrico $\left(0.006 \mathrm{M} \quad \mathrm{Fe}^{3+}\right)$ es ligeramente mayor que la de $0.010 \mathrm{M}$, aunque después de cinco horas la cantidad extraida de plata es la misma en ambos casos. Este comportamiento podria explicarse en base a las concentraciones de la especie $\mathrm{FeSO}_{4}\left[\mathrm{CS}\left(\mathrm{NH}_{2}\right)_{2}\right]^{+}$en la superficie de reacción $\left(R_{c}\right):$ la concentración de ésta es 10 veces menor inicialmente permitiendo una mayor concentración de especies de plata soluble, pero esta aumenta conforme transcurre el tiempo, al contrario de lo observado en la simulación a $0.010 \mathrm{M} \mathrm{Fe}^{3+}$. otros autores han reportado comportamientos no convencionales con plata (ver capitulo II). Definitivamente seria conveniente estudiar este fenómeno más detenidamente.

Con respecto a la variación de tiourea, se presentan los datos experimentales $y$ las simulaciones en la Figura 8 . Aqui se puede observar el efecto que causa un aumento en la concentración de ésta. A la concentración más baja de tiourea $\left(0.03 \mathrm{M} \operatorname{cS}\left(\mathrm{NH}_{2}\right)_{2}\right)$, la 
simulación predice que no hay precipitación del sólido $p_{2}$ a causa de la baja cantidad de su precursor. A $0.1 \mathrm{M}$, existe precipitación superficial y a $0.3 \mathrm{M}$, se llega a saturar la solución a un nivel de conversión menor que aquél obtenido en el experimento anterior. Este último resultado tiene un significado práctico muy importante: mientras en la lixiviación a $0.1 \mathrm{M}$ de tiourea la conversión sigue su tendencia hacia arriba $y$ puede llegar a conversión total (la predicción marca una conversión de $99 \%$ en 20 horas), la lixiviación usando 0.3M no llega a una conversión mayor de 72\%. Esto concuerda con el máximo en la velocidad de lixiviación a 0.1M de tiourea observado por Pesic y Seal (1990).

A un porciento de sólidos mayor (ver la Figura 9), el efecto de saturación es mayor, pero no en la medida que se esperaría, debido al agotamiento de la especie $\mathrm{FeSO}_{4}\left[\mathrm{CS}\left(\mathrm{NH}_{2}\right)_{2}\right]$ y las diferencias entre las distintas concentraciones de tiourea desaparecen. Aún así, las conversiones en ambos casos no llegan a la conversión total.

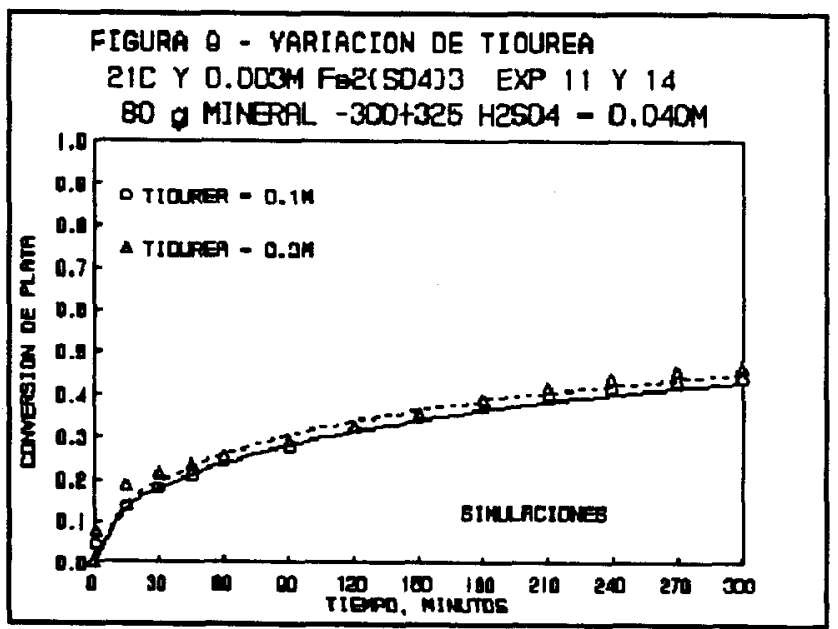
En el caso del experimento 11 a $0.1 M$ tiourea, la conversión máxima (saturación) predicha por el modelo a tiempos largos (ver Apéndice D) es de $68 \%$ y para el experimento 14 a $0.3 \mathrm{M}$ tiourea, es de 58\%. A pesar de que a $21^{\circ} \mathrm{C}$ no se obtiene una conversión alta debido a la 
Resultados y Discusión

baja solubilidad del $p_{2}$, a una temperatura mayor, como demostraron Khmel'nitskaya et al (1985), la solubilidad incrementa y se lograrian mayores conversiones.

El efecto de la temperatura, como se mencionó anteriormente, es mayor de lo esperado para un sistema controlado únicamente por difusión de los reactivos. Aunque el modelo modificado no predice formación de sólido a $35^{\circ} \mathrm{C}$, la forma de la curva experimental en la Figura 10 parece indicar que existe un acercamiento a saturación de la solución a esta temperatura. A los 21 y $3^{\circ} \mathrm{C}$, existe precipitación superficial y probablemente por esta razón el aumento mayor en la velocidad de lixiviación entre estas dos temperaturas.

En cuanto al contenido de sólidos, ésta es una variable que no deberia afectar a un sistema cuya reacción oxidativa es irreversible, hasta que empieza a agotarse el reactivo

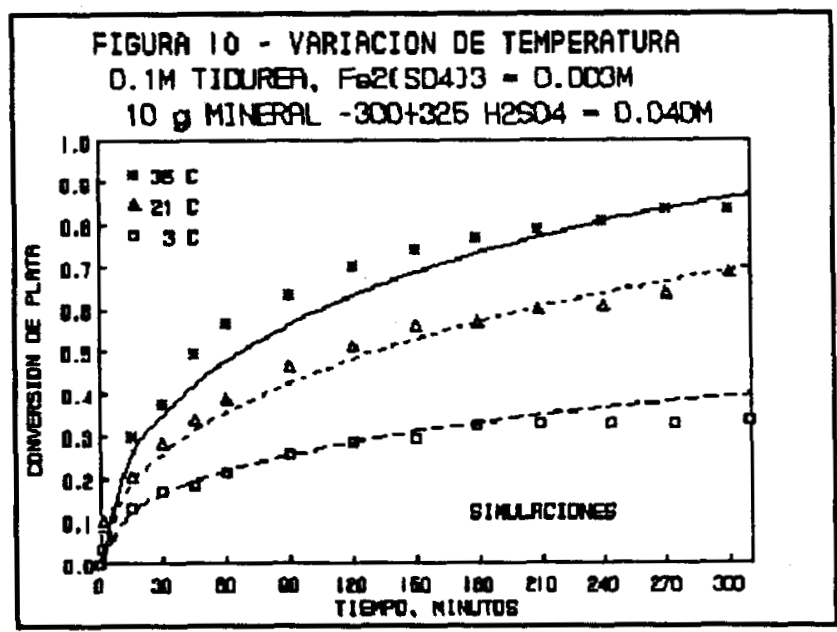
limitante. En cambio, cuando la reacción es reversible, tanto la conversión como la proporción líquido a sólido son importantes para la cinética de lixiviación. En la Figura 11 aparecen tres experimentos a las mismas condiciones iniciales de solución $y$ se observa que, incluso a muy bajas conversiones, existe una diferencia apreciable en la velocidad a pesar de que el reactivo limitante está lejos de agotarse y que la resistencia por transferencia de masa externa es despreciable (debido a la velocidad de agitación empleada en el reactor). 
Antes de terminar, es muy
ilustrativo comparar los perfiles de concentración de dos experimentos, el número 9 donde el modelo predice la formación del sólido en la superficie $\left(R_{c}\right)$ $y$ el número 15 donde no existe precipitación. En los dos, las condiciones son idénticas, excepto por la concentración de

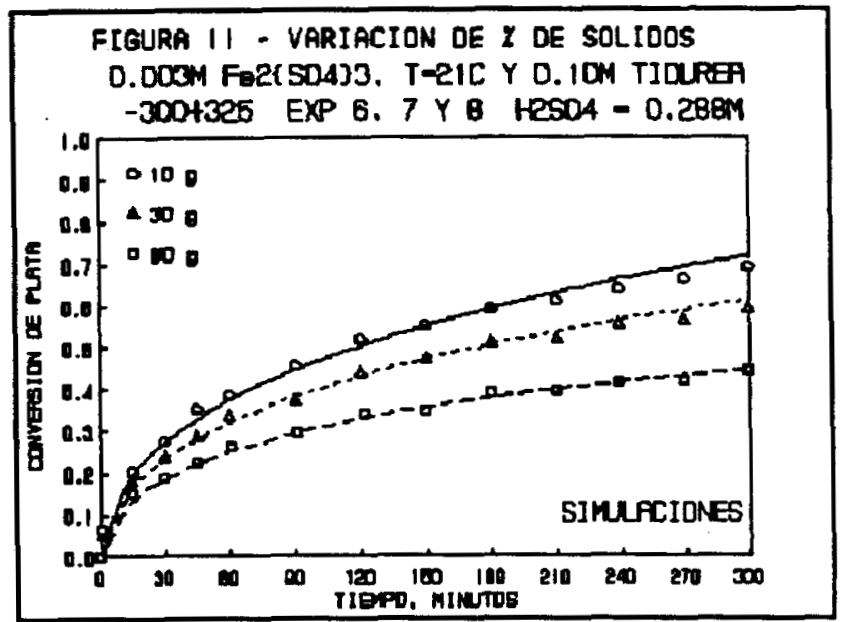
tiourea que en el primero es de $0.10 \mathrm{M} y$ en el segundo es de 0.03M. En ambos casos, los perfiles para la tiourea libre $y$ las especies relacionadas con los iones u03sulfato libre, hidronio y ferroso son prácticamente constantes por encontrarse en exceso. Donde se pueden ver las diferencias se sitúan en el reactivo limitante ( $\mathrm{Fe}^{3+} \mathrm{y}$ especies relacionadas) y los productos de plata. En un proceso controlado únicamente por difusión del reactivo limitante, el perfil de concentración debe reducirse, desde un valor igual al del seno de la solución hasta uno casi nulo en la frontera de reacción. En la Figura 12a, donde las concentraciones aparecen normalizadas con respecto a su

FIGURA 12a - PERFILES DE CONCENTRACION (Fa+3) D. DOON Fae[SDA]3. HESLA-0.DAON Y T = 21DC $100-300+325$ EXP Q Y 15 CONVERSION $=0.20$

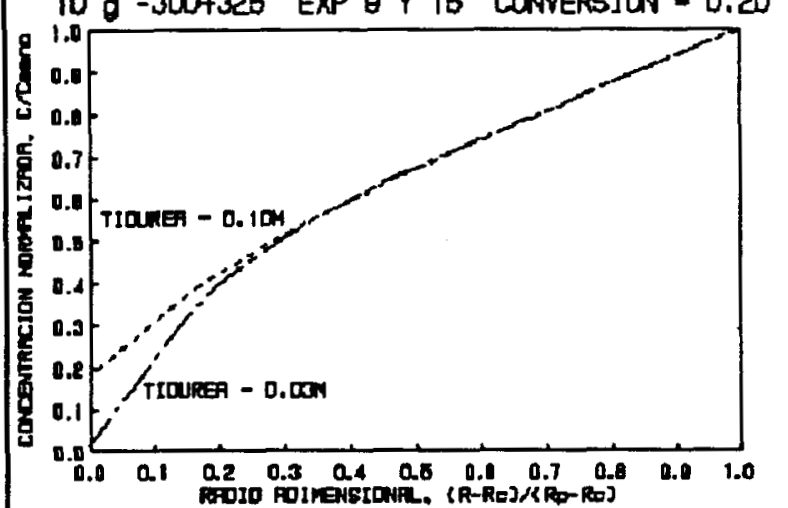
valor en el seno de liquido a $x$ $=0.20$, contra el radio adimensional, se puede ver que ésto se presenta en el caso del experimento $15\left(0.03 \mathrm{M} \mathrm{CS}\left(\mathrm{NH}_{2}\right)_{2}\right)$, pero no para el experimento 9 a $0.10 \mathrm{M}$. 
La fuerza impulsora del proceso en el caso de un control mixto (difusión de los reactivos y los productos) es la diferencia de concentraciones de las especies solubles de plata. En la Figura 12b, para la misma conversión que la figura anterior ( $X=$ $0.20)$, se observa que la diferencia es mayor para la especie, $\quad \mathrm{Ag}\left[\mathrm{CS}\left(\mathrm{NH}_{2}\right)_{2}\right]_{2}{ }^{+}, \quad$ del experimento $9(0.10 \mathrm{M})$ en donde hay precipitación. Para las otras especies de plata soluble,

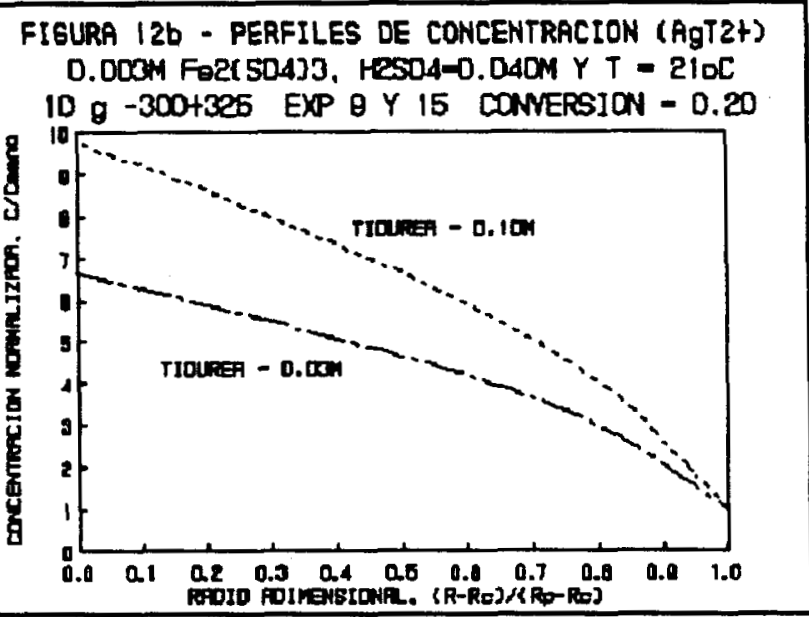
éstas siguen un comportamiento similar como se puede apreciar en la Tabla III y el Apéndice $D$.

TABLA III - COMPARACION DE LAS CONCENTRACIONES NORMALIZADAS DE PRODUCTOS DE PLATA EN LA FRONTERA DE REACCION $\left(R_{c}\right)$ PARA LOS EXPERIMENTOS 9 Y $15\left(21^{\circ} \mathrm{C}\right)$ CONVERSION DE PLATA $=0.20$

\begin{tabular}{|c|c|c|}
\hline ESPECIE & $\begin{array}{c}\text { EXP. } 9 \text { (0.1M) } \\
\text { CON PRECIPITACION }\end{array}$ & $\begin{array}{l}\text { EXP. } 15(0.03 \mathrm{M}) \\
\text { SIN PRECIPITACION }\end{array}$ \\
\hline $\begin{array}{c}\mathrm{Ag}^{+} \\
\mathrm{Ag}\left[\mathrm{CS}\left(\mathrm{NH}_{2}\right)_{2}\right]^{+} \\
\mathrm{Ag}\left[\mathrm{CS}\left(\mathrm{NH}_{2}\right)_{2}\right]_{2}^{+} \\
\mathrm{Ag}\left[\mathrm{CS}\left(\mathrm{NH}_{2}\right)_{2}\right]_{3}+ \\
\mathrm{Ag}\left[\mathrm{CS}\left(\mathrm{NH}_{2}\right)_{2}\right]_{4} \\
\mathrm{Ag}\left[\mathrm{CS}\left(\mathrm{NH}_{2}\right)_{2}\right]_{3_{+}}+ \\
\mathrm{Ag}\left[\mathrm{CS}\left(\mathrm{NH}_{2}\right)_{2}\right]_{6}\end{array}$ & $\begin{array}{r}10 \\
10 \\
10 \\
10 \\
10 \\
100 \\
89\end{array}$ & $\begin{array}{r}10 \\
5 \\
7 \\
5 \\
5 \\
70 \\
38\end{array}$ \\
\hline
\end{tabular}

En cuanto a la optimización de la lixiviación de este concentrado en particular con sulfato férrico y tiourea, por razones de velocidad $y$ de consumo de tiourea, es conveniente 
sugerir que se fijen las condiciones del experimento 11 , en $0.003 \mathrm{M}$ de sulfato férrico y $0.10 \mathrm{M}$ de tiourea a aproximadamente $10 \%$ de sólidos. Como se vió anteriormente, se puede prescindir del ácido sulfúrico porque el mismo sulfato férrico mantiene el pH de la solución. Si es permitido por la economia del proceso trabajar a una temperatura arriba de la ambiental, ésto mejoraría la cinética elevando la solubilidad del sólido, hasta una temperatura máxima de $35^{\circ} \mathrm{C}$.

Consumo de tiourea

Uno de los factores que ha impedido la comercialización del proceso de tioureación ácida es el alto consumo de tiourea. Se ha encontrado en experimentos preliminares que este puede reducirse en minerales sulfurados a un nivel aceptables ( $<58$ a $\left.0.003 \mathrm{M} \mathrm{Fe}_{2}\left(\mathrm{SO}_{4}\right)_{3}\right)$. En la medida que se incrementa la cantidad de sulfato férrico ó el potencial REDOX de la solución, arriba de $500 \mathrm{mV}$ SCE, la destrucción de tiourea es mayor y la plata puede precipitar desde la solución. Entonces, es importante notar que el uso excesivo de agente oxidante ó lixiviar minerales oxidados con un alto contenido de hematita $\left(\mathrm{Fe}_{2} \mathrm{O}_{3}\right)$ puede influir negativamente sobre la economia del proceso (Nomvalo, 1986). 
Resultados y Discusión

V.2 - PREDICCIONES DEL MODELO PARA OTRAS CONDICIONES DE SOLUCION

Efecto del pH a Concentraciones Menores de Tiourea

Dado el carácter aproximado de las constantes de formación Y el producto de solubilidad estimados para el modelo modificado, es dificil predecir con certeza a partir de que concentración de tiourea comienza la formación del precipitado sólido. No obstante este hecho, se puede investigar el comportamiento del modelo a bajas concentraciones de tiourea y sulfato férrico, donde el modelo predice una superficie libre de sólido. Aunque estas condiciones no son las comercialmente adecuadas para la extracción de plata en un tanque agitado por su cinética lenta, podrian servir para la lixiviación en montones o "heap leaching".

Para este caso se puede usar el modelo original que no considera la precipitación del sólido. Las simulaciones para un mineral sulfurado con 200 gramos de plata (como argentita) a $25 \%$ de sólidos de una solución de $0.03 \mathrm{M}$ tiourea $y 0.003 \mathrm{M}$ sulfato férrico para $\mathrm{pH} 1,1.5 \mathrm{Y} 1.9$, aparecen en la Figura 13 como conversión fraccional de plata contra tiempo adimensional.

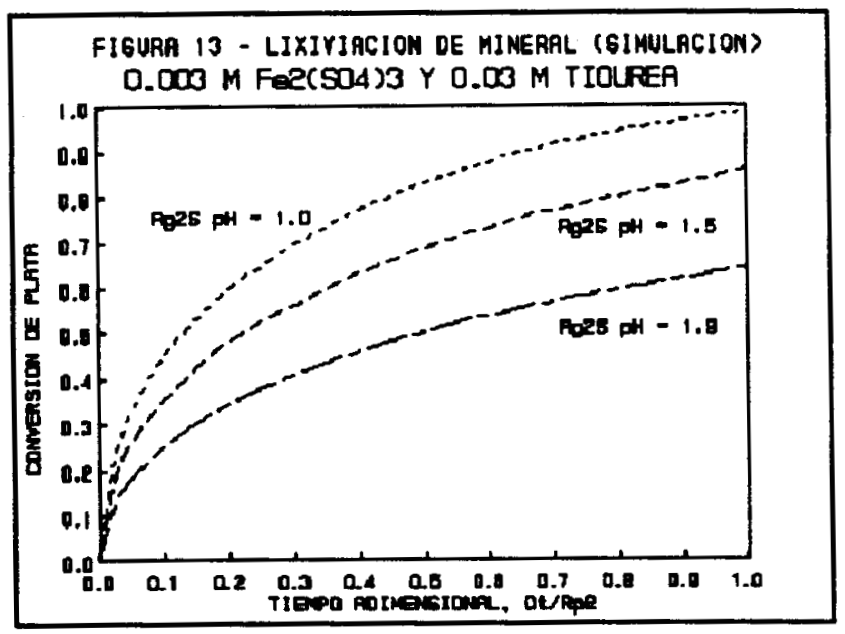

Peróxido de Hidrógeno

Aunque la presencia de hierro es inevitable en este sistema, el uso de otros agentes axidantes, como el peróxido de hidrógeno, reduciria la formación del sólido que impide la salida de la plata. 
En la Figura 14, se muestran los datos de Maldonado $y$ Lapidus (1991) para la extracción de plata empleando $0.001 \mathrm{M} \mathrm{H}_{2} \mathrm{O}_{2}$ a $\mathrm{pH}$ 1.0 con otra fracción $(-200+300$ malla) del mismo mineral, en función de la concentración de tiourea. Tanto el modelo original como el modificado predicen bien a los datos. Se

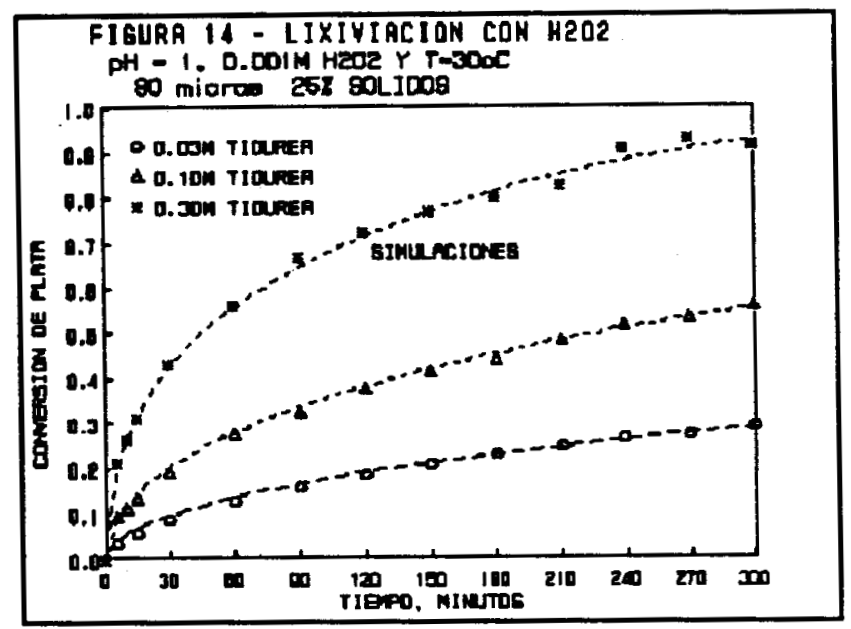
puede notar que a $0.3 \mathrm{M}$ de tiourea no existen indicios de saturación de la solución. Considerando que la cantidad de agente oxidante es tres veces menor, la extracción es más rápida a pesar de que el radio de particula es ligeramente mayor. En este caso, dado el efecto tan grande de la tiourea, que está en exceso con respecto al agente oxidante, no se puede concluir que el fenómeno limitante sea exclusivamente la difusión de los reactivos, sino también su influencia sobre la constante de equilibrio en el interfase [ecuación (45)].

El comportamiento con respecto al porciento de sólidos, Figura 15, demuestra que no hay diferencia alguna, que no sea por el agotamiento del agente oxidante. con una mayor cantidad de $\mathrm{H}_{2} \mathrm{O}_{2}$, la destrucción de tiourea puede ser apreciable, aunque la extracción de plata es más rápida (Maldonado y Lapidus, 1991).

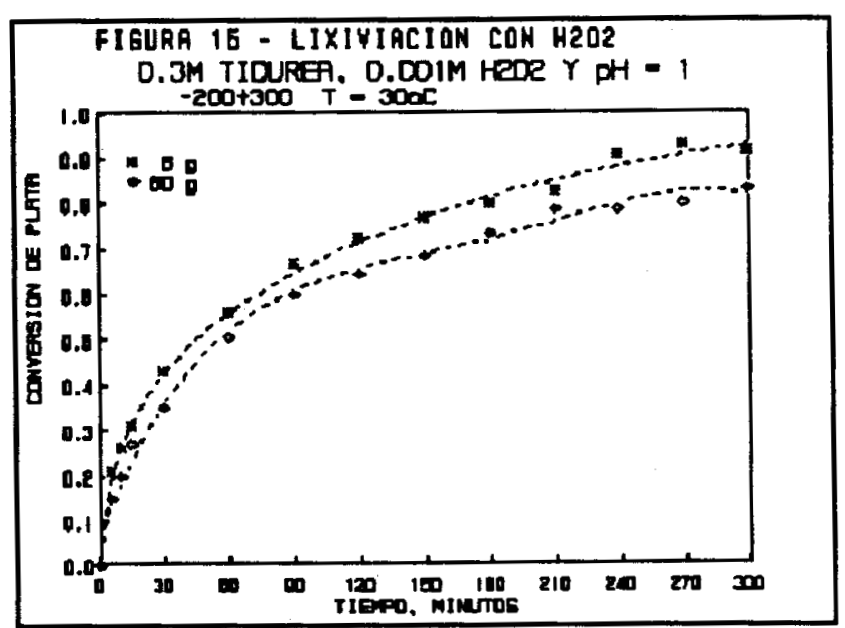


Resultados y Discusión

\section{V.3 - APLICABILIDAD A OTROS MINERALES}

Dado que las concentraciones de plata en el concentrado empleado en el presente estudio son muy elevadas, pueden surgir dudas referentes a la aplicabilidad del modelo a otros minerales con una composición distinta, por un lado, de plata $y$, por el otro, de otros metales. Como se ha visto anteriormente, si los fenómenos que limitan el proceso están bien descritos por el modelo, y los parámetros necesarios son conocidos, las predicciones de los datos experimentales son buenas. En el presente caso, la difusión de productos desde la frontera del núcleo hasta el seno de la solución controla la cínetica del proceso, independientemente de la cantidad de mineral empleada. Se puede comprobar que las concentraciones en la superficie de reacción serian prácticamente iguales para las condiciones idénticas de soluciones para otros minerales, entonces, 10 más probable es que se enfrentarian al mismo paso controlante. Las diferencias que podría ocasionar una ley menor de plata serian el menor consumo de agente oxidante, y que la solución no se saturara como en el presente caso.

Lo único que sería importante considerar son las diferencias en porosidad, a consecuencia de la extracción de otros metales, y el consumo que estos tendrian de tiourea y del agente oxidante. De hecho, Van Deventer et al (1990) mostraron que interacciones galvánicas entre oro $y$ otros fases minerales son minimas en el sistema de tiourea $y$, por analogía, se podría decir 10 mismo de la plata. Pesic y seal (1990) encontraron que, con la excepción de cobre que compleja con cuatro o seis moléculas de tiourea, la presencia de otros metales no afecta significativamente a la velocidad de lixiviación de plata. Especificamente, el contenido de hematita $\left(\mathrm{Fe}_{2} \mathrm{O}_{3}\right)$ en el mineral, además de disolverse rápidamente alterando la porosidad, cambiaria la cantidad del ión férrico, y consecuentemente el potencial REDOX de la solución. 
Resultados y Discusión

Desafortunadamente no existen estudios en la literatura con las carácteristicas adecuadas (con radio de partícula acotado $y$ midiendo la extracción de otros metales), que permitan confirmar las hipótesis planteadas con respecto a la aplicabilidad a otros minerales. Esto se reserva para otro estudio dado que no es el objetivo del presente trabajo. 
Conclusiones

\section{CAPITULO VI - CONCLUSIONES}

A partir del estudio experimental de la lixiviación de plata de un concentrado sulfurado, con sulfato férrico y tiourea, se puede concluir lo siguiente:

1) Para la mayoría de las condiciones de la solución estudiadas (0.1-0.3M tiourea, 0.003-0.010M sulfato férrico, 0.024-0.288M ácido sulfúrico $Y$ temperaturas menores de los $\left.35^{\circ} \mathrm{C}\right)$, el paso controlante de la cinética es la disolución de un sólido formado (probablemente el $\left.\mathrm{Ag}_{2} \mathrm{SO}_{4}\left[\mathrm{CS}\left(\mathrm{NH}_{2}\right)_{2}\right]_{6}\right)$ sobre la superficie en donde ocurre la reacción. Es posible que a bajas concentraciones de tiourea $(0.03 \mathrm{M})$, este precipitado no se forme.

2) Debido a 10 anterior, es conveniente llevar a cabo la lixiviación a una temperatura lo más alta posible $\left(35^{\circ} \mathrm{C}\right)$ para elevar la solubilidad del precipitado.

3) Las condiciones óptimas encontradas para la lixiviación de plata de concentrados sulfurados en el presente sistema son $0.003 \mathrm{M}$ sulfato férrico y $0.1 \mathrm{M}$ tiourea, porque maximiza la velocidad de extracción, manteniendo bajo el consumo de tiourea.

Como apreciación general, en comparación con estudios anteriores utilizando peróxido de hidrógeno en lugar de sulfato férrico como agente oxidante, resulta más rápida la lixiviación y menos propensa a la formación de precipitados, conservando las mismas ventajas de bajo consumo de tiourea.

Con respecto a los modelos planteados para estudiar este sistema: 
4) El modelo original sólo produce ajustes coherentes a los datos experimentales cuando no existe precipitación del sólido (bajas concentraciones de sulfato férrico y tiourea). En estos casos, la calidad de la predicción es muy buena; además, el modelo se puede emplear para predecir la lixiviación de plata de un concentrado con peróxido de hidrógeno y tiourea.

5) El modelo modificado, debido al carácter aproximado de las constantes de complejación $y$, seguramente, a un planteamiento inconsistente con respecto al producto de solubilidad del sólido formado, sólo permite predicciones ligeramente mejores que el modelo anterior. Aún asi, es capaz de predecir la precipitación superficial a partir del cálculo de las concentraciones locales de las todas las especies y la saturación de la solución lixiviante.

En general, se puede concluir que los modelos planteados en este trabajo constituyen un avance sobre anteriores modelos $y$ son buenos candidatos para ser incorporados, como la parte medular, a un simulador de reactores multiparticulas para procesos industriales de lixiviación. Una de las grandes virtudes de contar con modelos de lixiviación como los presentados es que, siendo de tipo fenomenológico, permiten la posibilidad de optimizar las condiciones de solución para otros minerales.

La continuación del presente trabajo tiene dos vertientes: por un lado, estudiar las mismas variables de solución en otros minerales de composición distinta $Y$, por el otro, ajustar $Y / O$ determinar con precisión los valores de las constantes de complejación utilizadas en el modelo modificado. Ambas medidas permitirian mejorar las predicciones $y$ la generalidad de dicho modelo. 
Bibliografia

\section{BIBLIOGRAFIA}

Becker, E., Knothe, M. Y Lobel, J. (1983), "Gold Recovery from Non-Metallic Secondary Raw Materials by Leaching with Thiourea and Adsorption on Ion-Exchangers", Hydrometallurgy, 11, pp. 265-275.

Bilston, D. W., La Brody, S. R. Y Woodcock, J. T. (1984), "Gold and silver Leaching from an oxidized Gold ore with Thiourea under Controlled Conditions", Aus. I. M. M. Symp. Series, 36 (Extractive Metall. Symp.), pp. 51-60.

Bodson, F. J. J. (1981), "Metal Recovery from sulfur Containing Material", French Patent FR 2,476,137. [CA 96:89165k] .

Bromley, L. A. (1973), "Thermodynamic Properties of strong Electrolytes in Aqueous Solutions", AIChE Journal, 19(2), pp. 313-320.

Charley, w. R. (1983), "Hydrometallurgical Extraction of Precious Metals using Thiourea", Pract. Hydromet $183,7^{\text {th }}$ Annual Symp. on Uranium and Precious Metals, pp. 75-81.

Chen, C. K., Long, T. N. Y Wan, C. C. (1980), "A study of the Leaching of Gold and silver by Acidothioureation", Hydrometallugy, 5 , pp. 207-212.

Contreras-Bustos, R. (1988), "Leaching of $\mathrm{Ag}_{2} \mathrm{~S}$ with Thiourea using Ferric Ion as Oxidant in Acidic Medium", Research Report, SENKEN. 


\section{Bibliografia}

Corro-León, J. (1985), "Altas Y Bajas de la Minería Mexicana", Información Cientifica y Tecnológica, No. 7(102), pp.39-40.

Deschenes, G. y Ghali, E. (1988), "Leaching of Gold from a Chalcopyrite Concentrate by Thiourea", Hydrometallurgy, $\underline{20}$, pp. 179-202 .

Dupuis, C. Y and Ghali, E. (1989), Thiourea Stability during Gold Leaching from Chalcopyrite concentrate", Precious and Rare Metal Technologies, Eds. A. E Torma Y I. H. Gundiler, Elsevier, Amsterdam, pp. 225-240.

Fuerstenau, M. C., Chen, C. C., Han, K. N. Y Palmer, B. R. (1986), "Kinetics of Galena Dissolution in Ferric Chloride Solutions", Metallurgical Transactions B, 17B, pp. 415-423.

Gabra, G. (1984), "A Kinetic study of the Leaching of Gold from Pyrite Concentrate using Acidified Thiourea" in Precious Metals: Mining, Extraction and Processing, Eds. V. Kudryk, D. A. Corrigan and W. W. Liang, Metallurgical Society of AIME, New York, pp. 145-172.

Geankoplis, c. J. (1972), Mass Transport Phenomena, Holt, Rinehart and winston, Inc., New York, pp. 127-130.

Gornostaeva, T. D., Khmel'nitskaya, O. D., Panchenko, A. E. Y Lodeishchikov, V. V. (1986), "A Potentiometric study of Thiourea Complexes of silver in sulphuric Acid Solutions", Russian Journal of Inorganic Chemistry, 31(1), pp. 64-66. 


\section{Bibliografia}

Goto, S., Ogawa, O., Asakura, I. Y Nakamura, S. (1985), The Leaching of Gold and silver from ore with Thiourea in Sulfuric Acid Solutions", J. of the Mining and Metallurgical Institute of Japan, 101(1164), pp. 75-79.

Groenewald, T. (1977), "Potential Applications of Thiourea in the Processing of Gold", J. S. Afr. Inst. Min. Metall., 77(11), pp. 217-223.

Groenewald, T. Y Jones, B. M. (1971), "Determination of Gold in Solutions of Thiourea", Analytical Chemistry, $\underline{43}(12), \mathrm{pp}$. 1689-91.

Groenewald, T. (1975), "Electrochemical studies on Gold Electrodes in Acid Solutions of Thiourea containing Gold(I) Thiourea Complex Ions", J. Appl. Electrochem., 5(1), pp. 7178 .

Groenewald, T. (1976), "The Dissolution of Gold in Acid Solutions of Thiourea", Hydrometallurgy, 1(3), pp. 277-290.

Gubailovskii, V. V. et al. (1972), "An Examination of the Kinetics of Silver-Chloride Dissolution in Aqueous Solutions of Thiourea", Tsvet. Metal., $\underline{45}(5)$, pp. 36-37. [Citado por Hiskey (1981)]

Habashi, F. (1970), Principles of Extractive Metallurgy, Vol. 2: Hydrometallurgy, Gordon and Breach, New York, pp. 24-39. 


\section{5}

Bibliografia

Hiskey, J. B. (1983), "Thiourea Leaching of Gold and Silver Technology Update and Additional Applications", Pract. Hydromet $183,7^{\text {th }}$ Annual Symp. On Uranium and Precious Metals, pp. 173-179.

Hiskey, J. B. (1981), "Thiourea as Lixiviant for Gold and Silver" in Gold and Silver: Leaching, Recovery and Economics, Eds. W. J. Schlitt, w. C. Larson and J. B. Hiskey, SME, Littleton, pp. 83-91.

Hisshion, R. J. Y Waller, C. G. (1984), "Recovering Gold with Thiourea", Mining Magazine, 151(3), pp. 237-43.

Huyhua, J. C. Y Gundiler, H. I. (1986), "Kinetics of Leaching Gold and silver in Acidic Thiourea Solutions" en Hydrometallurgical Reactor Design and Kinetics, Eds. R. G. Bautista, R. J. Wesely y G. W. Warren, The Metallurgical Society, Warrendale, pp. 247-264.

Huyhua, J. C., Zegarra, C. R. y Gundiler, H. I. (1988), "A Comparative study of oxidants on Gold and Silver Dissolution in Acidic Thiourea Solutions" en Precious Metals 189, Eds. M. C. Jha $y$ S. D. Hill, The Minerals, Metals and Materials Society, pp. 287-303. 


\section{Bibliografia}

Khmel'nitskaya, O. D., Gornostaeva, T. D., Panchenko, A. F. Y Lodeishchikov, V. V. (1985), "Solubility of $\left[\mathrm{Ag}_{2} \text { (Thio) }_{3}\right]_{\mathrm{SO}_{4}} \mathrm{Y}$ [Ag,ThiO) $\left.{ }_{3}\right]_{2} \mathrm{SO}_{4}$ in Solutions of Sulphuric Acid and Thiourea", Russian Journal of Inorganic Chemistry, 30(2), pp. 319-320.

Lapidus, G. T. Y Mosqueira, M. L. (1988), "Effect of Product Solubility on the Leaching Kinetics of Non-Porous Minerals", Hydrometallurgy, 20(1), pp. 49-64.

Levenspiel, O. (1972), Chemical Reaction Engineering, $2^{\text {nd }}$ Ediion, John Wiley and Sons, Inc., New York, pp. 361-377.

Levich, V. G. (1973), Physicochemical Hydrodynamics, Prentice Hall, New York.

Lodeishchikov, v. V., Shamis, L. A., Kakovskii, I. A Y Khmel'nitskaya, O. D. (1975), "Investigation of the Kinetics of the Dissolution of Silver in Aqueous Solutions of Thiourea", Izv. Vyssh. Uchebn. Zaved., Tsvetn. Metall., $\underline{2}$, pp. 77-81.

Maldonado, G. T. Y Lapidus, G. T. (1991), "Lixiviación de Plata usando Peróxido de Hidrógeno y Tiourea", Avances en Ingenieria Química 1990, Eds. R. Fonseca et al., Academia Mexicana de Investigación y Docencia en Ingeniería Química, México, en prensa.

Maslowska, J. (1969), "Mixed Complexes of Iron(III)", Zesz. Nauk. Politechn. Lodz, Chem., 20, pp. 5-114. [Citado por Groenewald, 1977]. 


\section{Bibliografia}

Monhemius, A. J. (1987), "Recent Advances in the Treatment of Refractory Gold Ores", II Meeting of the Southern Hemisphere on Mineral Technology, Rio de Janiero, Brazil, 25-29 Mayo 1987.

Moussoulos, L., Potamianos, N. Y Kontopoulos, A. (1984), "Recovery of Gold and Silver from Arseniferrous Pyrite Cinders by Acidic Thiourea Leaching", in Precious Metals: Mining, Extraction and Processing, Eds. V. Kudryk, D. A. Corrigan and W. W. Liang, Metallurgical Society of AIME, New York, pp.

Nomvalo, Z. T. (1986), "Thiourea Leaching of witwatersrand ore", Gold 100: Proceedings of the International Conference on Gold, Vol. 2: Extractive Metallurgy of Gold, S. Afr. I. M. M., Johannesburg, pp. 565-577.

Ospanov, Kh. K., Abetova, E. K. Y Songina, O. A. (1971), "Dissolution of Silver using Thiourea", Izv. Akad. Nauk. Kaz. SSSR, Ser. Khim, 21(6), pp. 5-9. [CA 76:104417b] .

Panchenkov, A. F., Kakovskii, I. A., Shamis, L. A. y Khmel'nitskaya, O. D. (1975), "Dissolution Kinetics of Gold, Silver and their Alloys in Aqueous Thiourea", Izv. Akad. Nauk. SSSR, Met., 6, pp. 32-7. [CA $84: 138813 \mathrm{~h}$ ].

Panchenkov, A. F, et al (1976), "Theoretical Basis of the Dissolution of Gold, Silver and their Alloys in Acid Thiocarbamide Solutions", Trzisy Dokl. - Vses. Soveshch. Khim. Anal. Tekhnol, Blagorodn, Met., (1), 14. [CA 90: 12879]. 
Bibliografia

Pashkov, G. L., Kopanev, A. M., Kulenov, A. S., Andreev, A. I. Y Fedorov, V. A. (1984), "The Stability of Thiourea Complexes of Silver(I) in Aqueous Sodium Sulfate Solutions", Russian Journal of Inorganic Chemistry, 29(4), pp. 584-586.

Pesic, B. Y Seal, T. (1988), "Dissolution of silver with Thiourea: The Rotating Disk Study" en Precious Metals 189, Eds. M. C. Jha y S. D. Hill, The Minerals, Metals and Materials Society, pp. 261-285.

Pesic, B. Y Seal, T. (1990), "A Rotating Disk Study of Silver Dissolution with Thiourea in the Presence of Ferric Sulfate", Metallurgical Transactions B, 21B(June), pp. 419-427.

Plaksin, I. N. Y Kozhukhova, M. A. (1960), "Dissolution of Gold and Silver in Solutions of Thiourea", Sb. Nauchn. Tr., Inst. Tsvetn. Metal., 33, pp. 107-119. [CA 57:10,888e]

Preisler, P. W. y Berger, L. (1947), "Oxidation-Reduction Potentials of Thioldithio systems: Thiourea-Formamidine Disulfide", J. of A. C. S., 69, pp. 322-25.

Pyper, R. A. Y Hendrix, J. L. (1981b), "Extraction of Gold from Finely Disseminated Gold ores by Acidic Thiourea Solutions" in Extraction Metallurgy '81, I. M. M., London, pp. 57-75.

Pyper, R. A. Y Hendrix, J. L. (1981a), "Extraction of Gold from Carlin type ore using Thiourea" in Gold and silver: Leaching, Recovery and Economics, Eds. W. J. Schlitt, w. C. Larson and J. B. Hiskey, SME, Littleton, pp. 93-108. 


\section{Bibliografia}

Rath, P. C., Paramguru, R. K. Y Jena, P. K. (1988), "Kinetics of Dissolution of Sulphide Minerals in Ferric Chloride Solution. I: Dissolution of Galena, Sphalerite and Chalcopyrite", Trans. Instn. Min. Metall. (Sect. c: Mineral Process. Extr. Metall.) 97 (September), pp. C150-C158.

Robinson, R. A. Y Stokes, R. H. (1959), Electrolyte Solutions, $2^{\circ}$ Edition, Butterworth Scientific Publishers, London.

Royston, D., Spencer, P. A. Y Winborne, D. A. (1984), "Alkaline oxidation of Pyrite in Gold Recovery", Aus. I. M. M. Symp. Series, 36 (Extractive Metall. Symp), pp. 61-7.

Rubcumintra, T. Y Han, K. N. (1990), "The Effect of Concentration and Temperature on Diffusivity of Metal Compounds", Metallurgical Transactions B, 21B(June), pp. 429-438.

Sandberg, R. G. Y Huiatt, J. L. (1986), "Recovery of Silver, Gold and Lead from a Complex Sulfide ore using Ferric Chloride, Thiourea and Brine Leach Solutions", Bureau of Mines Report of Investigations, RI 9022.

Sandberg, R. G. Y Huiatt, J. L. (1986), "Ferric chloride, Thiourea and Brine Leach Recovery of $\mathrm{Ag}, \mathrm{Au}$ and $\mathrm{Pb}$ from Complex Sulfides", J. of Metals, 38(6), pp. 18-22.

Schulze, R. G. (1984a), "New Aspects in Thiourea Leaching of Precious Metals", J. of Metals, 36(6), pp. 62-65.

Schulze, R. G. (1984b), "Determination of Thiourea in Leach Liquors by Titration with Potassium Iodate", Hoja de Información de SKW Trostberg Aktiengesellschaft, 19 enero. 
Bibliografía

Schulze, R. G. (1986), "Thiourea Leaching of Precious Metals", Erzmetall, 39(2), pp. 57-59.

Shen, J. Y Smith, J. M. (1965), "Diffusional Effects in Gas-Solid Reactions", Ind. and Eng. Chem., Fundamentals, 4(3), pp. 293301.

Shivrin, G. N. Y Shivrina, E. M. (1976), "Kinetics of the Electrochemical Reduction of Thiourea Complexes of Metales", Soviet J. Nonferr. Metals Res., 4 (5), pp. 192-4.

Smith, R. M. Y Martell, A. E. (1976), Critical stability Constants, Vol. 4: Inorganic Complexes, Plenum Press, New York, pp. 1-14, 79-85 y 104-112.

Smith, R. M. Y Martell, A. E. (1982), Critical stability Constants, Vol. 5: Organic and Inorganic Complexes, Plenum Press, New York, pp.

Sohn, H. Y. Y Szekely, J. (1972), "The Effect of Reaction Order in Non-Catalytic Gas-Solid Reactions", Can. J. of Chem. Eng., 50(October), pp. 674-676.

Sohn, H. Y. (1979), "Fundamentals of the Kinetics of Heterogeneous Reaction Systems in Extractive Metallurgy" en Rate Processes of Extractive Metallurgy, Eds. Sohn, H. Y. Y Wadsworth, M. E., Plenum Press, New York, pp. 1-51. 


\section{Bibliografia}

Songina, O. A., Ospanov, Kh. K., Muldagalieva, I. Kh. Y Salnikova, S. D. (1971), "Dissolution of Gold using Thiourea in Hydrochloric Acid Medium", Izv. Akad. Nauk. Kaz. SSR, Ser. Khim., 21(3), pp. 9-11. [CA 75:917152].

Stephen, W. I. Y Townsend, A. (1966), "The Reactions of Silver(I) Ions with Organic Compounds containing the HN-C-S Grouping. Part II. Some Thiourea Derivatives", Journal of the Chemical Society (A), pp. 166-168.

Tataru, S. (1968), "Dissolution of Gold in Thiourea Solutions", Rev. Roum. Chim., 13, pp. 891-7.

Taylor, P. R., de Matos, M. Y Martins, G. P. (1983), "Modelling of Noncatalytic Fluid-Solid Reactions: The Quasi-steady State Assumption", Met. Trans. B, 14B(March), pp. 49-53.

Tellez, J., Rosas, G., Bedolla, E. Y Tavera, F. J. (1986), "Estudio de la Extracción de oro y plata de un Mineral Aurifero: Lixiviación Acida en Tiourea - Adsorción en Carbón Reducción Carbotérmica", VIII Encuentro de Investigación Metalúrgica, 10-12 sept., pp. 121-141.

Turner, M. J. (1986), "Gold Ore Leaching with Thiourea", Long Term Project Report, Imperial College of Science and Technology.

Van Deventer, J. S. J., Reuter, M. A., Lorenzen, L. Y Hoff, P. J. (1990), "Galvanic Interactions during the Dissolution of Gold in Cyanide and Thiourea Solutions", Mineral Engineering, $3(6)$, pp. 589-597. 


\section{Bibliografia}

Villadsen, J. V. Y Michelsen, M. L. (1978), solution of Differential Equation Models by Polynomial Approximation, Prentice Hall, Englewood Cliffs, New Jersey.

Warren, G. M., Henein, H. Y Jin, Z. M. (1985), "Reaction Mechanism for the Ferric Chloride Leaching of Sphalerite", Metall. Trans. B, 16B, pp.

Wen, C. Y. (1968), "Noncatalytic Heterogeneous Solid-Fluid Reaction Models", Ind. and Eng. Chem., 60(9), pp. 34-54.

Won, c. w. Y Cho, T. R. (1985), "The Dissolution of Gold and Silver in Acidic Solutions of Thiourea", J. of the Korean Institute of Metals, $\underline{23}(5)$, pp. 495-500.

Yagi, S. Y Kunii, D. (1955), "studies on Combustion of Carbon Particles in Flames and Fluidized Beds", $5^{\text {th }}$ International Symposium on Combustion, Reinhold, New York, pp. 231-244.

Zegarra, C. R., Huyhua, J. C. Y Gundiler, I. H. (1989), Thiourea Leaching of Manganiferous Gold-silver ores", Precious and Rare Metal Technologies, Eds. A. E Torma Y I. H. Gundiler, Elsevier, Amsterdam, pp. 209-223. 


\section{NOMENCLATURA}

(AT) = Concentración total de agente oxidante

Bal = Acumulación de plata en la solución

$\mathrm{BalA}=$ Acumulación adimensional de plata $=\mathrm{Bal} / \mathrm{C}_{1}{ }^{\circ}$

$c_{j}=$ Concentración de la especie $i$

$c_{j}{ }^{0}=$ Concentración inicial de la especie $i$

$c_{j}=$ Concentración adimensional $=c_{j} / c_{1}{ }^{\circ}$

$D_{j} \quad=$ Difusividad efectiva de la especie $i$

(FT) = Concentración total de hierro

$\mathrm{k}_{\mathrm{f}} \quad$ = Tasa de la reacción de formación de una especie

$k_{d} \quad$ = Tasa de la reacción de desaparición de una especie

$k_{m a}=$ Coeficiente de transferencia de masa desde el seno de la solución hasta la superficie de la particula

$\mathrm{k}_{\mathrm{S}} \mathrm{c}_{\mathrm{A}}=$ Tasa de reacción de primer orden para el agente oxidante

$\mathrm{N}_{\mathrm{A}}$ = Densidad de flujo del agente oxidante

(PT) = Concentración total de plata

$\mathrm{R} \quad=$ Radio

$R_{c} \quad=$ Radio del núcleo decreciente (frontera de la reacción

$R_{p} \quad=$ Radio de la particula

de oxidación)

$R_{f j}$ = Velocidad de reacción de formación de la especie $j$

$R_{\mathrm{dj}}$ = Velocidad de reacción de desaparición de la especie $j$

$\{S T\}=$ Concentración total de sulfato

$t \quad=$ Tiempo

$\{\mathrm{TT}\}=$ Concentración total de tiourea

$\mathrm{x}$ = Extracción o conversión fraccional de plata

$\alpha \quad=$ Función de primer orden para la destrucción de tiourea a

las condiciones de la solución (experimental)

$\Delta_{\mathrm{j}} \quad=$ Difusividad adimensional $=\mathrm{D}_{\mathrm{j}} / \mathrm{D}_{\mathrm{q}}$

$\Phi=$ Radio del núcleo adimensional $=R_{c} / R_{p}$

$\tau=$ Tiempo adimensional $=D_{1} t / R_{p}{ }^{2}$

$\eta \quad=$ Coordenada espacial adimensional $=\left(R-R_{c}\right) /\left(R_{p}-R_{c}\right)$

$\epsilon \quad=$ Porosidad de la capa de inertes

$\rho_{M} \quad=$ Densidad molar de la plata en el mineral 
Apéndice A

\section{APENDICE A - PROCEDIMIENTOS EXPERIMENTALES ESPECIFICOS}

\section{A.1. - CONDICIONES EMPLEADAS PARA EL ANALISIS POR ABSORCION ATOMICA}

El intervalo de concentración y los parámetros que se utilizaron para determinar las concentraciones de plata y cobre por el espectrofotómetro de absorción atómica son los siguientes:

$\begin{array}{ccc}\text { Concentraciones } & \text { Longitud de onda } & \text { Apertura } \\ \text { ppm } & \mathrm{nm} & \mathrm{nm}\end{array}$

$\begin{array}{llll}\mathrm{Ag} & 3-12 & 338.5 & 0.5 \\ \mathrm{Cu} & 2-8 & 324.7 & 0.5\end{array}$

Se añadió solución lixiviante a los estándares de ambos metales de acuerdo a la dilución de la muestra, para asegurar la igualdad del fondo, especialmente en la concentración de fierro, elemento que produce más interferencias. Para el blanco, se usó la solución lixiviante a la misma dilución.

A.2 - CUANTIFICACION DE TIOUREA TOTAL

Se oxida la tiourea al disulfuro formamidina con iodato de potasio en soluciones ácidas mediante la siguiente reacción (Schulze, 1984b):

$6 \mathrm{CS}\left(\mathrm{NH}_{2}\right)_{2}+\mathrm{KIO}_{3}+6 \mathrm{H}^{+} \Longrightarrow 3\left[\mathrm{CS}\left(\mathrm{NH}_{2}\right)_{2}\right]_{2}^{2+}+3 \mathrm{H}_{2} \mathrm{O}+\mathrm{KI}$

Para asegurar que la totalidad de la tiourea está en su forma reducida antes de llevar a cabo la titulación, se pone la muestra 


\section{Apéndice A}

en contacto con polvo de zinc y ácido sulfúrico. Posteriormente, se filtra el exceso de zinc, enjuagando bien el medio filtrante para evitar pérdidas de tiourea. Se titula la muestra con una solución valorada de iodato de potasio en presencia de un indicador de yodo (almidón). El valor titulado corresponde a la cantidad de tiourea más disulfuro formamidina en la muestra original. 
Apéndice B

APENDICE B - ADIMENSIONALIZACION DE LAS ECUACIONES DEL MODELO

B.1. - BaLANCE de las especies en EL SENo DE LA SOLUCion (BAL)

El parámetro Bal tiene como propósito corregir a cada tiempo las concentraciones de las especies en solución a causa de la reacción oxidativa. Este término es de suma importancia para sistemas: 1) en donde no es muy grande el exceso del agente oxidante, 2) la reacción de oxidación llega al equilibrio y/o 3) la salida de productos está restringida por saturación de la solución.

$$
\begin{aligned}
\mathrm{Bal} & =\frac{\text { mol } \mathrm{Ag} \text { originalmente en el mineral }}{\text { litro de solución lixiviante }} \\
& =\frac{\text { Contenido de } \mathrm{Ag} \text { en el mineral }}{\text { Peso molecular de } \mathrm{Ag}} \times \begin{array}{c}
\text { Densidad de la pulpa } \\
\text { de lixiviación }
\end{array} \\
& =\frac{\mathrm{g} \mathrm{Ag}}{\text { ton mineral }} \frac{10^{-3} \mathrm{ton} \mathrm{mineral}}{\mathrm{kg} \text { mineral }} \frac{\mathrm{mol} \mathrm{Ag}}{\mathrm{g} \mathrm{Ag}} \frac{\mathrm{kg} \text { mineral }}{\mathrm{lt} \text { solución }}
\end{aligned}
$$

Multiplicando $\mathrm{Bal}$ por la conversion, $x$, se obtiene la concentración de plata en el seno de la solución. Asimismo, para obtener la concentracíon de las otras especies participantes en la reacción de oxidación, se multiplica Bal*X por el coeficiente estequiométrico correspondiente.

\section{B.2. - JUSTIFICACION DE LA AGRUPACION DE ESPECIES}

Cuando una especie química j está en equilibrio dinámico con otras, hay una continua transformación, de acuerdo con el ambiente químico local $y$ mediante las reacciones de complejación, que provoca la formación y la desaparición de la especie. 


$$
€ \frac{\partial C_{j}}{\partial t}=\frac{D_{j}}{R^{2}} \frac{\partial}{\partial R}\left[R^{2} \frac{\partial C_{j}}{\partial R}\right]+R_{f_{j}}-R_{d_{j}}
$$

7 donde, $R_{\mathrm{fj}}$ = velocidad de reacción de formación de $j$

$$
\begin{aligned}
\mathrm{R}_{\mathrm{dj}} & =\text { velocidad de reacción de desaparición de } \mathrm{j} \\
\epsilon & =\text { porosidad de la capa de inertes }
\end{aligned}
$$

Por ejemplo, se escribe el balance para la especie $7, \mathrm{FeSO}_{4}^{+}$(ver pagina 19), en la siguiente forma:

$$
€ \frac{\partial C_{7}}{\partial t}=\frac{D_{7}}{R^{2}} \frac{\partial}{\partial R}\left[R^{2} \frac{\partial C_{7}}{\partial R}\right]+R_{f_{7}}-R_{d_{1}}
$$

Como se considera que la especie 7 está en equilibrio con las especies 1 y $2, \mathrm{Fe}^{3+}$ y $\mathrm{SO}_{4}{ }^{2}$, respectivamente y que la transformación es muy rápida, las velocidades de formación y de desaparición están relacionadas con la constante de equilibrio de la reacción (23), 0 sea:

$$
K_{23}=\frac{\left[\mathrm{FeSO}_{4}^{+}\right]}{\left[\mathrm{Fe}^{3+}\right]\left[\mathrm{SO}_{4}^{2-}\right]}=\frac{k_{f}}{k_{\mathrm{d}}}
$$

donde, $k_{\mathrm{f}}=$ tasa de la reacción de formación

$k_{d}=$ tasa de la reacción de desaparición

La ecuación de difusión para el $\mathrm{FeSO}_{4}{ }^{+}$queda entonces como sigue:

$$
\epsilon \frac{\partial C_{7}}{\partial t}=\frac{D_{7}}{R^{2}} \frac{\partial}{\partial R}\left[R^{2} \frac{\partial C_{7}}{\partial R}\right]+k_{f}\left[\mathrm{Fe}^{3+}\right]\left[\mathrm{SO}_{4}^{2-}\right]-k_{d}\left[\mathrm{FeSO}_{4}^{+}\right]
$$


Apéndice B

Si se lleva a cabo un procedimiento análogo para las demás especies de hierro $\left(c_{1}, c_{5}, c_{8} Y c_{11}\right)$ y se suman, se puede demostrar que se cancelan los términos de velocidad de formación $y$ desaparición.

\section{B.3. - ADIMENSIONALIZACION DE LA ECUACION DE DIFUSION}

La ecuación de difusión para un núcleo decreciente es un típico problema de frontera móvil, y para facilitar su resolución es conveniente llevar a cabo un cambio de variable que la inmovilice.

$$
\left.\varepsilon \frac{\partial C_{j}}{\partial t}\right|_{R}=\frac{D_{j}}{R^{2}} \frac{\partial}{\partial R}\left[R^{2} \frac{\partial C_{j}}{\partial R}\right]_{t} \quad R_{c}<R<R_{p}
$$

Se usan las siguientes variables adimensionales (Taylor et al, 1983):

$$
\eta=\frac{R-R_{c}}{R_{p}-R_{c}} \quad \phi=\frac{R_{C}}{R_{p}} \quad C_{j}=\frac{C_{j}}{C_{1}^{0}} \quad \tau=\frac{D_{1} t}{R_{\mathrm{p}}^{2}} \quad \Delta_{j}=\frac{D_{j}}{D_{1}}
$$

Se utilizó arbitrariamente el ión férrico libre $\left(C_{1} Y D_{1}\right)$ como referencia para adimensionalizar la concentración, el tiempo y las difusividades efectivas de los demás compuestos.

Sustituyendo las últimas tres variable, se obtiene:

$$
\left.\frac{\partial C_{j}}{\partial \tau}\right|_{R}=\frac{\Delta_{j} R_{p}^{2}}{\epsilon}\left[\frac{\partial^{2} C_{j}}{\partial R^{2}}+\frac{2}{R} \frac{\partial C_{j}}{\partial R}\right]_{\tau}
$$




\section{Apéndice B}

Dado que la variable adimensional, $\eta$, depende de $R Y R_{c}(t)$, entonces,

$$
\begin{gathered}
\left.\frac{\partial C_{j}}{\partial \tau}\right|_{R}=\left.\frac{\partial C_{j}}{\partial R}\right|_{\eta}+\left.\left.\frac{\partial C_{j}}{\partial \eta}\right|_{\tau} \frac{\partial \eta}{\partial \tau}\right|_{R} \\
\partial R=R_{p}(1-\phi) \partial \eta \\
\left.\frac{\partial \eta}{\partial \tau}\right|_{R}=\frac{-(1-\eta)(1-\phi)}{(1-\phi)^{2}} \frac{d \phi}{d \tau}
\end{gathered}
$$

donde, $d \Phi / d \tau=$ Velocidad de encogimiento del núcleo

Sustituyendo,

$$
\begin{gathered}
\left.\epsilon \frac{\partial C_{j}}{\partial \tau}\right|_{\eta}=\frac{\Delta_{j}}{(1-\phi)^{2}}\left[\frac{\partial^{2} C_{j}}{\partial \eta^{2}}+\frac{\partial C_{j}}{\partial \eta}\left[\frac{2(1-\phi)}{\eta(1-\phi)+\phi}+\frac{\epsilon(1-\eta)(1-\phi)}{\Delta_{j}} \frac{d \phi}{d \tau}\right]\right] \\
0<\eta<1
\end{gathered}
$$

La velocidad de encogimiento del núcleo [ecuación (50)] adimensionalizada toma la siguiente forma:

$$
\frac{d \phi}{d \tau}=\frac{c_{1}^{0}}{\rho_{M}} \frac{\Delta_{P T}}{(1-\phi)} \frac{\partial C_{P T}}{\partial \eta}
$$

La conversión se define como sigue:

$$
X=(1-\Phi)^{3}
$$


APENDICE $C$ - PROGRAMA Y CONSTANTES USADAS PARA RESOLVERLO

C.1. - PROGRAMA (NO INCLUYE LOS SUBRUTINAS DE PAQUETE)

DIAGRAMA DE BLOQUES

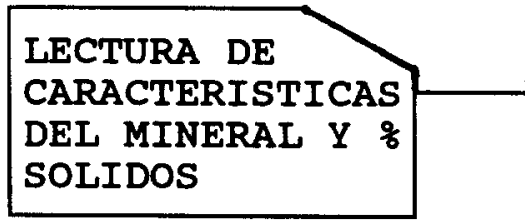

CALCULO DE LA POROSIDAD Y LA TORTUOSIDAD
LECTURA DE

LA TEMPERATURA $Y$

LAS CONSTANTES DE

EQUILIBRIO, $\triangle H, E T C$. A

FUERZA IONICA $\left(I_{0}\right)=1$

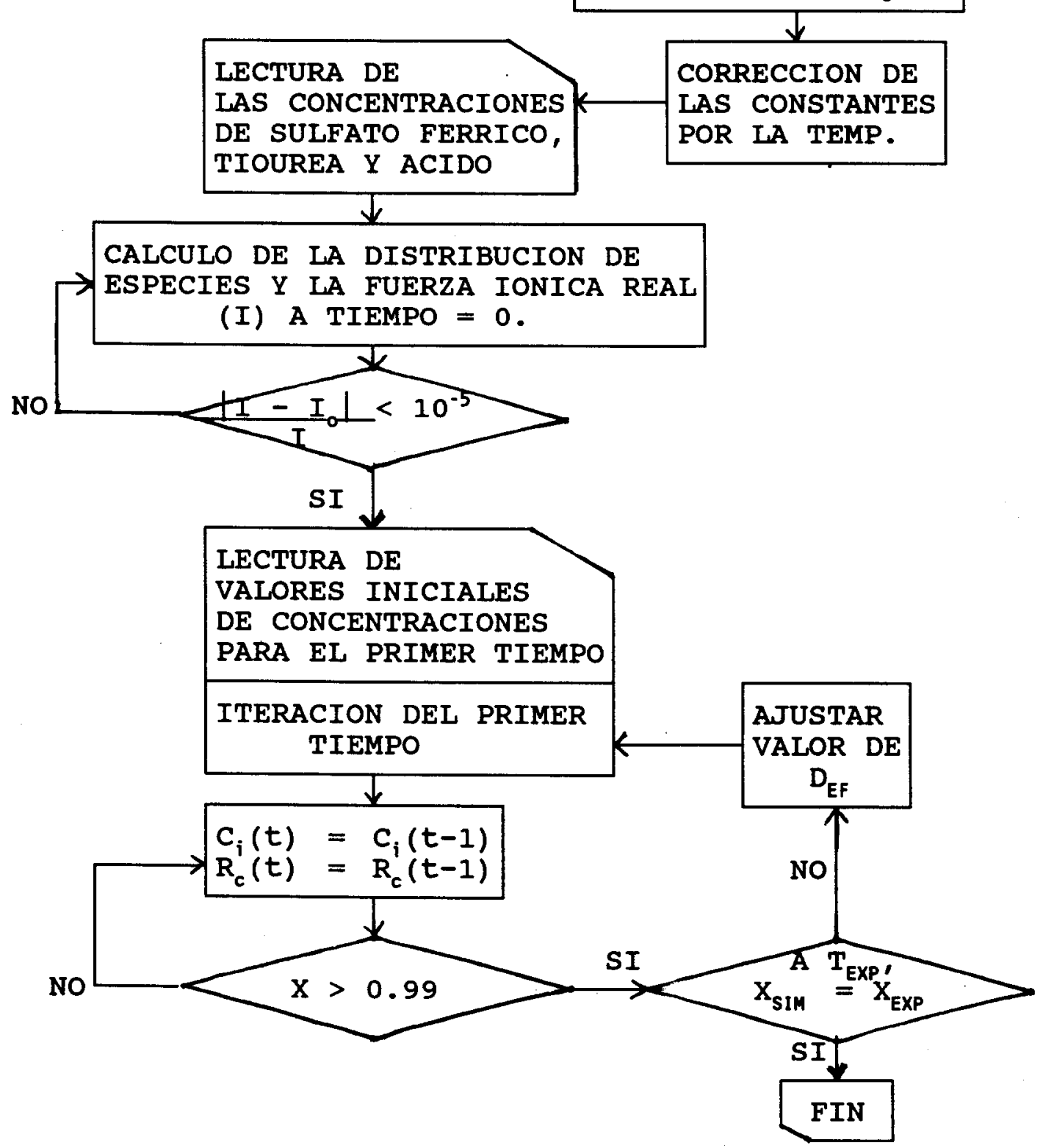


Apéndice C

PROGRAM PLATA (INPUT, OUTPUT, TAPE5=INPUT , TAPE7 , TAPE17, TAPE8, +TAPE18=OUTPUT)

C

C

C

C

C

C

C

C

C

C

C

C

C

C

C

C

C

C

EL PROGRAMA PLATA CALCULA LA CINETICA DE LIXIVIACION EN TIEMPO REAL DE PLATA A PARTIR DE PLATA METALICA O ARGENTITA EN MINERALES UTILIZANDO SOLUCIONES ACUOSAS DE TIOUREA Y SULFATO FERRICO O PEROXIDO DE HIDROGENO. SE BASA EN UN MODELO DE NUCLEO DECRECIENTE TRANSIENTE CON LA REACCION DE OXIDACION EN EQUILIBRIO A LA FRONTERA DEL NUCLEO Y EQUILIBRIO DINAMICO EN IA CAPA DE INERTES DE 17 ESPECIES.

DIMENSION DIF1 (10), DIF2 (10), ROOT (10), $\operatorname{AM}(10,10)$

$+, \operatorname{VECT}(10), \operatorname{BM}(10,10)$

+ ,DIF3 (10), XINTP (10), RAIZ (10), BOUND (180)

DIMENSION X(91), $\mathrm{FX}(61,61), \mathrm{F}(61), \mathrm{XO}(91), \mathrm{XR}(19)$,

$+\operatorname{ENT}(18), \mathrm{EQ}(8), \mathrm{BO}(8), \mathrm{ZAS}(8), \mathrm{ZBS}(8), \mathrm{ZCS}(8), \mathrm{TA}(8), \mathrm{TB}(8)$,

$+\mathrm{XOR}(12), \mathrm{DX}(61), \mathrm{FUNC}(4), \operatorname{ETA}(10), \operatorname{FDX}(61), \mathrm{WK}(1200)$,

$+\operatorname{FAC}(8), \operatorname{FACS}(8), \operatorname{FEC}(6), \operatorname{FFEC}(6), \mathrm{C}(5), \operatorname{PAR}(161), \mathrm{CHG}(51)$

$+, \operatorname{AJT}(3), \operatorname{AJX}(3)$

COMMON/HYB/A, B, ROOT

COMMON/POLI / DIF 1, DIF2, DIF3

COMMON/ INTER/NIN , DIN

COMMON/DAT1/ ALFA, BETA

COMMON/FUN/IX (4), NO, N1, F9

COMMON / DIFU / DELB , DELC, DELD , DELE , DELF , DELG, DELH , DELI,

+DELJ , DELK , DELL , DELM , DELN , DELP , DELO, DELQ, DELR

COMMON/SYST/G, AGOXID, COX, FETOT, STOT, THIO, AS , SOLIQ, GPTAG

+ , EPT , RADPAR, DEF, PEROX

COMMON / NPUNTOS / NT , AM , BM, ETA , IA , NF

COMMON/CONEQ/XR

COMMON/IT/ITC

EXTERNAL FCN

LECTURA DE LAS CARACTERISTICAS DEL MINERAL: ITDM = TIPO DE MINERAL, 1) PLATA METALICA Y 2) ARGENTITA; GPTAG = GRAMOS DE PLATA POR TONELADA DE MINERAL; DENMIN = DENSIDAD DE MINERAL EN G/ML; RADPAR = RADIO DE LA PARTICULA EN CM; POROS = POROSIDAD ORIGINAL DEL MINERAL (PUEDE INCLUIR OTRAS ESPECIES QUE SE LIXI VIAN RAPIDAMENTE); SOLIQ = PROPORCION DE SOLIDO A LIQUIDO EN KG MINERAL/LITRO DE SOLUCION.

READ (17, *) ITDM, GPTAG , DENMIN , RADPAR, POROS , SOLIQ

PARAMETROS PARA CALCULAR LOS PUNTOS Y LAS MATRICES DE COLOCACION ORTOGONAL

$\mathrm{NO}=1$

$\mathrm{N} 1=1$ 


\section{Apéndice $C$}

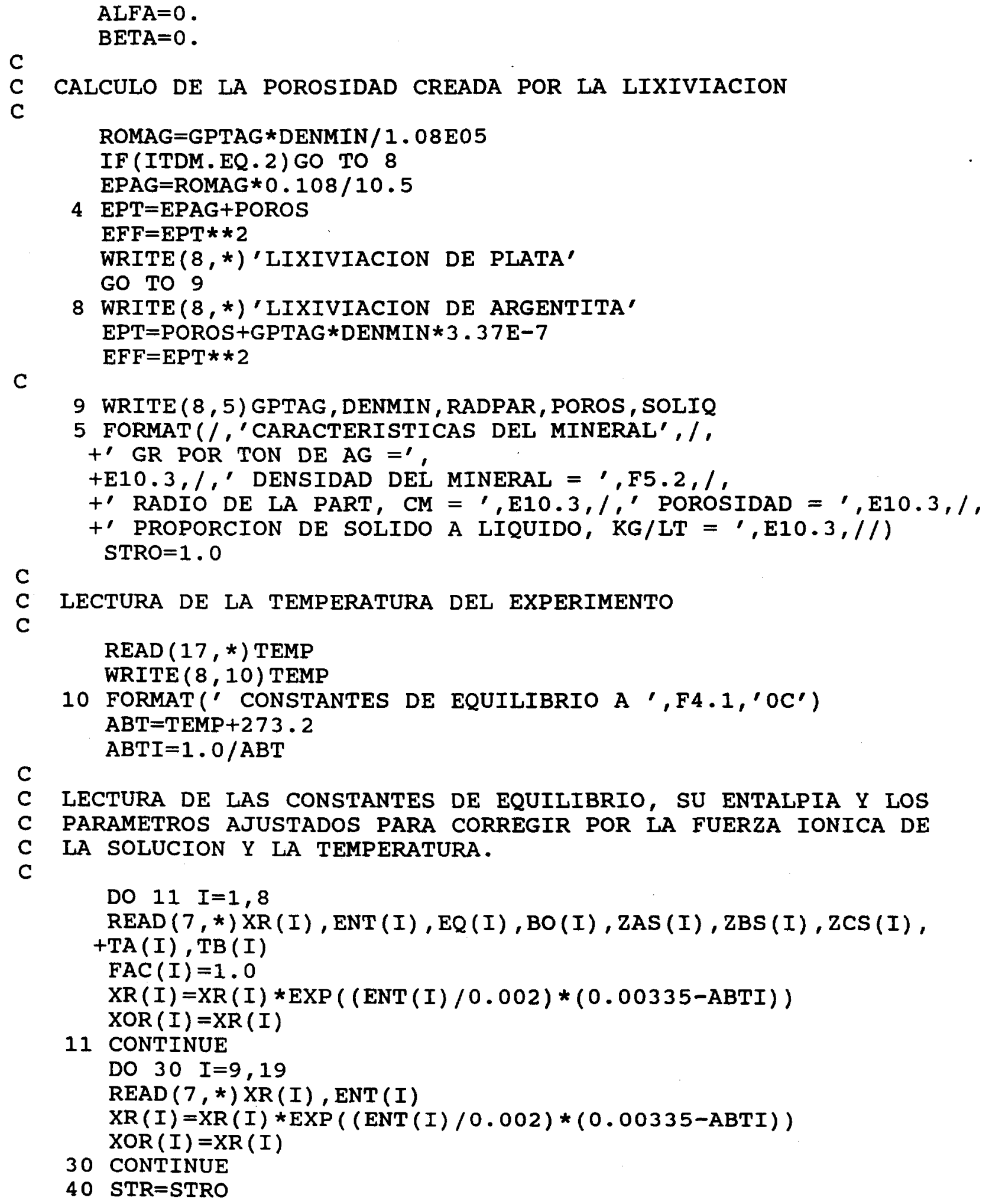


Apéndice $C$

C
$C$
$C$
$C$
$C$
$C$
$C$

$C$
$C$
$C$
$C$
$C$
$C$

LECTURA DE LAS VARIABLES DEL EXPERIMENTO (CONCENTRACIONES EN MOLES/LITRO) : FESUL = SULFATO FERRICO; THIO = TIOUREA; PH = PACIDO; PTOT = PRESION TOTAL EN BARES; AS = FUNCION DE PRIMER ORDEN PARA LA DESTRUCCION DE TIOUREA (EXPERIMENTAL) EN (MINUTO) $-1 ;$ PEROX = PEROXIDO DE HIDROGENO.

READ (17, *) FESUL, THIO, PH , PTOT, AS , PEROX $\mathrm{PHO}=\mathrm{PH}$

ITERACION PARA CALCULAR Y CORREGIR LAS CONSTANTES DE EQUILIBRIO POR LA FUERZA IONICA REAL DE LA SOLUCION $Y$ VERIFICACION DE LA CANTIDAD DE ACIDO ANADIDO Y EL PH REAL. ESTIMACION INICIAL DE LA DISTRIBUCION DE ESPECIES.

FETOT $=2$. $0 *$ FESUL

HENRYI $=$ EXP $(-8.669-2.32 \mathrm{E} 03 * \mathrm{ABTI}+5.06 \mathrm{E} 05 * \mathrm{ABTI} * * 2)$

$\mathrm{COX}=0.21 * \mathrm{PTOT} * \mathrm{HENRYI} * 55.6$

AGOXID $=$ FETOT $+2.0 *$ PEROX

G=AGOXID/ROMAG

$\mathrm{PH}=\mathrm{PHO}$

$\mathrm{IPH}=0$

45 IF (IPH.GT. 0) $\mathrm{PH}=\mathrm{PH}+0.5$

$I P H=I P H+1$

$\mathrm{B}=\mathrm{XR}(8) /(10.0 * *(-\mathrm{PH}))$

$\mathrm{HL}=10.0 * *(-\mathrm{PH})$

WRITE $(8,50)$ FESUL , PEROX, THIO, PH , G , AS

50 FORMAT $(/ /, '$ CONCENTRACION INICIAL DE FE2 (SO4) $3=1$, E10.3, $/$, $+'$ CONCENTRACION INICIAL DE H2O2 = , E10.3, $/$,

$+{ }^{\prime}$ CONCENTRACION INICIAL DE TIOUREA $=,$, E10.3,, ,

$+\quad$ GAMA $=$, E10.3,l,

$+'$ VELOCIDAD DE DESCOMPOSICION DE TIOUREA = TIME*', E10.3,//) $\mathrm{ZOACID}=0.5 * \mathrm{HL} *(1.0+\mathrm{XR}(7) * \mathrm{FESUL} * 3.0 * 0.01)$

$I T=0$

60 STOT $=3.0 *$ FESUL+ZOACID

$\mathrm{S}=(\mathrm{STOT}-\mathrm{FETOT}) /(1.0+\mathrm{XR}(7) * \mathrm{HL})$

$A Q=-S * X R(2)$

$\mathrm{BQ}=\mathrm{XR}(2) * \mathrm{~S} *(\mathrm{THIO}-\mathrm{FETOT}-\mathrm{XR}(1) / \mathrm{XR}(2))-1.0$

$\mathrm{CQ}=\mathrm{THIO}+\mathrm{XR}(1) * \mathrm{~S} * \mathrm{THIO}$

$\mathrm{TO}=(-\mathrm{BQ}-\mathrm{SQRT}(\mathrm{BQ} * * 2-4.0 * \mathrm{AQ} * \mathrm{CQ})) /(2.0 * \mathrm{AQ})$

$\mathrm{FE}=\mathrm{FETOT} /(1.0+\mathrm{S} *(\mathrm{XR}(1)+\mathrm{TO} * \mathrm{XR}(2)))$

$70 \mathrm{FEC}(1)=\mathrm{XR}(1) * \mathrm{FE} * \mathrm{~S}$

$\operatorname{FEC}(2)=\mathrm{XR}(2) * \mathrm{FE} * \mathrm{~S} * \mathrm{TO}$

$\operatorname{FEC}(3)=\mathrm{XR}(3) * \mathrm{FE} * \mathrm{~B}$

$\operatorname{FEC}(4)=\mathrm{XR}(4) * \mathrm{FE} * \mathrm{~B} * * 2$

FEC $(5)=X R(5) * F E * * 2 * B * * 2$

$\mathrm{FEC}(6)=\mathrm{XR}(6) * \mathrm{FE} * * 3 * \mathrm{~B} * * 4$

$\mathrm{DHSO}=\mathrm{XR}(7) * \mathrm{HL} * \mathrm{~S}$

C

GO TO 135 


\section{Apéndice C}

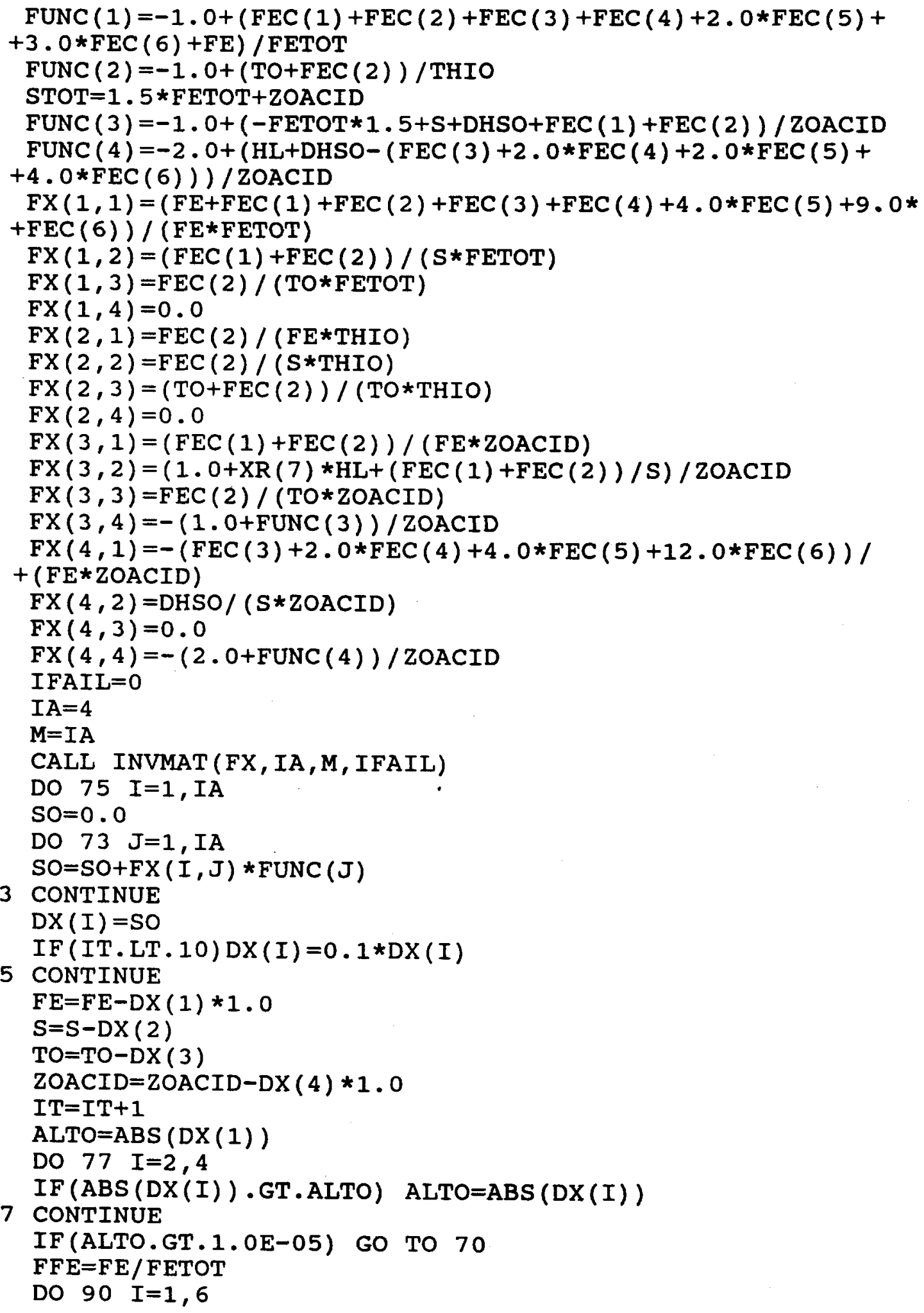


Apéndice C

90 CONTINUE

STRR $=0.5 *(9.0 *$ FE $+F E C(1)+F E C(2)+4.0 *$ FEC ( 3$)+F E C(4)+16.0 *$

$+\mathrm{FEC}(5)+25.0 * \mathrm{FEC}(6)+\mathrm{DHSO}+4.0 * \mathrm{~S}+\mathrm{B}+\mathrm{HL})$

IF (ABS ( (STRR-STR)/STR) . LT.1.0E-05) GO TO 120

DO $100 \mathrm{~K}=1,8$

FACS $(\mathrm{K})=\mathrm{ZAS}(\mathrm{K}) * * 3 * \mathrm{TA}(\mathrm{K}) *(\mathrm{STRR} /((\mathrm{ZAS}(\mathrm{K})+1.5 * \mathrm{STRR}) * * 2)-$

$+1.0 /((\mathrm{ZAS}(\mathrm{K})+1.5) * * 2))+\mathrm{ZBS}(\mathrm{K}) * \star 3 * \mathrm{~TB}(\mathrm{~K}) *(\mathrm{STRR} /((\mathrm{ZBS}(\mathrm{K})+$

$+1.5 * \mathrm{STRR}) * * 2)-1.0 /((\mathrm{ZBS}(\mathrm{K})+1.5) * * 2))-\mathrm{ZCS}(\mathrm{K}) * * 3 *(\mathrm{STRR} /$

$+((\mathrm{ZCS}(\mathrm{K})+1.5 * \mathrm{STRR}) * * 2)-1.0 /((\mathrm{ZCS}(\mathrm{K})+1.5) * * 2))$

$A D=S Q R T(S T R R) /(1.0+S Q R T(S T R R))-0.5$

$\mathrm{FAC}(\mathrm{K})=10.0 * *(298.2 / \mathrm{ABT} *(0.511 * \mathrm{EQ}(\mathrm{K}) * \mathrm{AD}+(0.06+0.6 * \mathrm{BO}(\mathrm{K})) *$

$+\mathrm{FACS}(\mathrm{K})+\mathrm{BO}(\mathrm{K}) *(1.0-\mathrm{STRR})))$

100 STR $=$ STRR

GO TO 70

$120 \operatorname{WRITE}(8,130) \mathrm{FE}, \operatorname{FEC}(1), \operatorname{FEC}(2), \operatorname{FEC}(3), \operatorname{FEC}(4), \operatorname{FEC}(5), \operatorname{FEC}(6)$, +FFE, FFEC (1), FFEC (2), FFEC (3), FFEC (4), FFEC (5), FFEC (6), STRR

130 FORMAT ( $6 \mathrm{X},{ }^{\prime} \mathrm{FE}+3^{\prime}, 6 \mathrm{X}$, 'FESO4+', 3X, 'FESO4(TH) +', 3X, 'FEOH+2', $+4 \mathrm{X},{ }^{\prime} \mathrm{FE}(\mathrm{OH}) 2+^{\prime}, 2 \mathrm{X}$, ' $^{\mathrm{FE} 2}(\mathrm{OH}) 2+4^{\prime}, 1 \mathrm{X},{ }^{\prime} \mathrm{FE} 3(\mathrm{OH}) 4+5^{\prime}, /, 7 \mathrm{E} 11.3, /$, $+7 F 11.4,1, '$ FUERZA IONICA DE LA SOLUTION $=\prime$, F5.3)

FEOXID $=$ FEC ( 3$)+$ FEC ( 4$)+$ FEC ( 5$)+$ FEC (6)

FETOTR=FETOT-FEOXID

WRITE $(8,132)$ FETOTR

C

132 FORMAT (1X,' CANTIDAD DE ION FERRICO UTILIZABLE =', E10.3,/)

C ASIGNACION DE LA DIFUSIVIDADES LIBRES DE LAS DIFERENTES

C ESPECIES Y CALCULO DE LAS DIFUSIVIDADES ADIMENSIONALES.

C

135 DIFLA $=0.8 E-05$

$\mathrm{DEF}=\mathrm{EFF} * \mathrm{DIFLA}$

WRITE $(8, *)$ 'DIFUSIVIDAD EFECTIVA DE FE+3 =',DEF

$\mathrm{DIFLG}=0.4 \mathrm{E}-05$

DIFLH $=1.84 \mathrm{E}-06$

DIFLC $=5.0 \mathrm{E}-05$

$D I F L I=2.0 \mathrm{E}-05$

$D I F L B=1.0 E-05$

DIFLD $=1.1 \mathrm{E}-05$

DIFLJ $=5.5 \mathrm{E}-06$

DIFLF $=4.7 \mathrm{E}-06$

DIFLE $=1.0 \mathrm{E}-05$

DIFLK $=0.9 \mathrm{E}-05$

DIFLL $=4.7 \mathrm{E}-06$

DIFLM $=4.7 \mathrm{E}-06$

DIFLN $=4.7 \mathrm{E}-06$

DIFLP $=4.7 \mathrm{E}-06$

DIFLO $=2.4 \mathrm{E}-05$

$D I F L Q=2.35 \mathrm{E}-06$

DIFLR $=2.35 \mathrm{E}-06$ 
Apéndice C

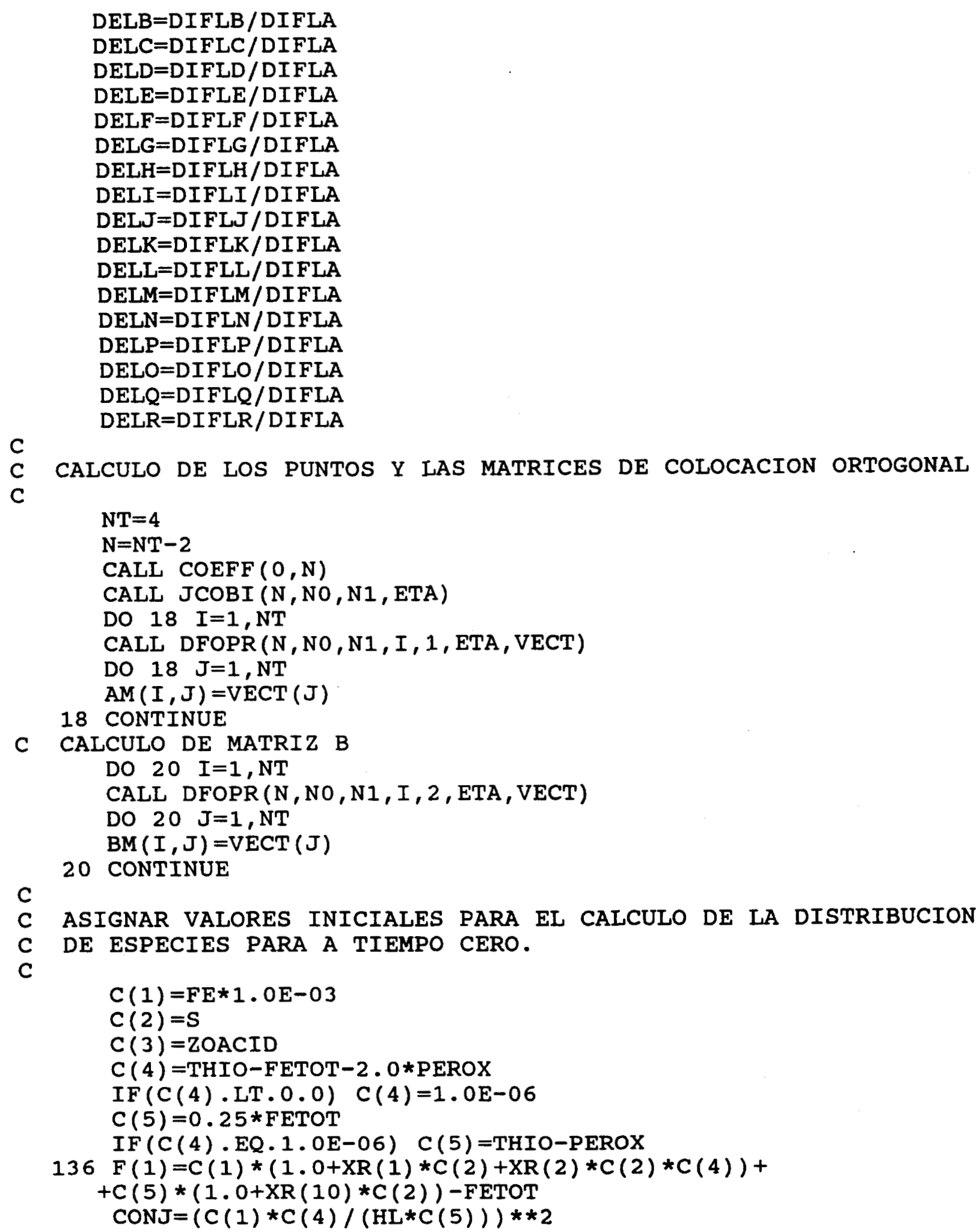

C

C CALCULO DE LOS PUNTOS Y LAS MATRICES DE COLOCACION ORTOGONAL

C

$\mathrm{NT}=4$

$\mathrm{N}=\mathrm{NT}-2$

CALL COEFF $(0, N)$

CALL JCOBI (N, NO, N1, ETA)

DO $18 I=1, N T$

CALL DFOPR (N, NO, N1, I, 1, ETA, VECT)

DO $18 \mathrm{~J}=1, \mathrm{NT}$

$\operatorname{AM}(I, J)=\operatorname{VECT}(J)$

18 CONTINUE

C CALCULO DE MATRIZ B

DO $20 \mathrm{I}=1, \mathrm{NT}$

CALL DFOPR(N, NO, N1, I, 2, ETA, VECT)

DO $20 \mathrm{~J}=1$, NT

$\operatorname{BM}(I, J)=\operatorname{VECT}(J)$

20 CONTINUE

c

C ASIGNAR VALORES INICIALES PARA EL CALCULO DE LA DISTRIBUCION

C DE ESPECIES PARA A TIEMPO CERO.

C

$C(1)=F E * 1.0 E-03$

$C(2)=S$

$C(3)=$ ZOACID

$C(4)=$ THIO-FETOT $-2.0 *$ PEROX

IF $(C(4)$.LT. 0.0$) \quad C(4)=1.0 E-06$

$C(5)=0.25 *$ FETOT

IF $(C(4) \cdot E Q \cdot 1.0 E-06) \quad C(5)=$ THIO-PEROX

$136 F(1)=C(1) *(1.0+X R(1) * C(2)+X R(2) * C(2) * C(4))+$

$+C(5) *(1.0+X R(10) * C(2))-F E T O T$

CONJ $=(C(1) * C(4) /(H L * C(5))) * * 2$ 


\section{Apéndice $C$}

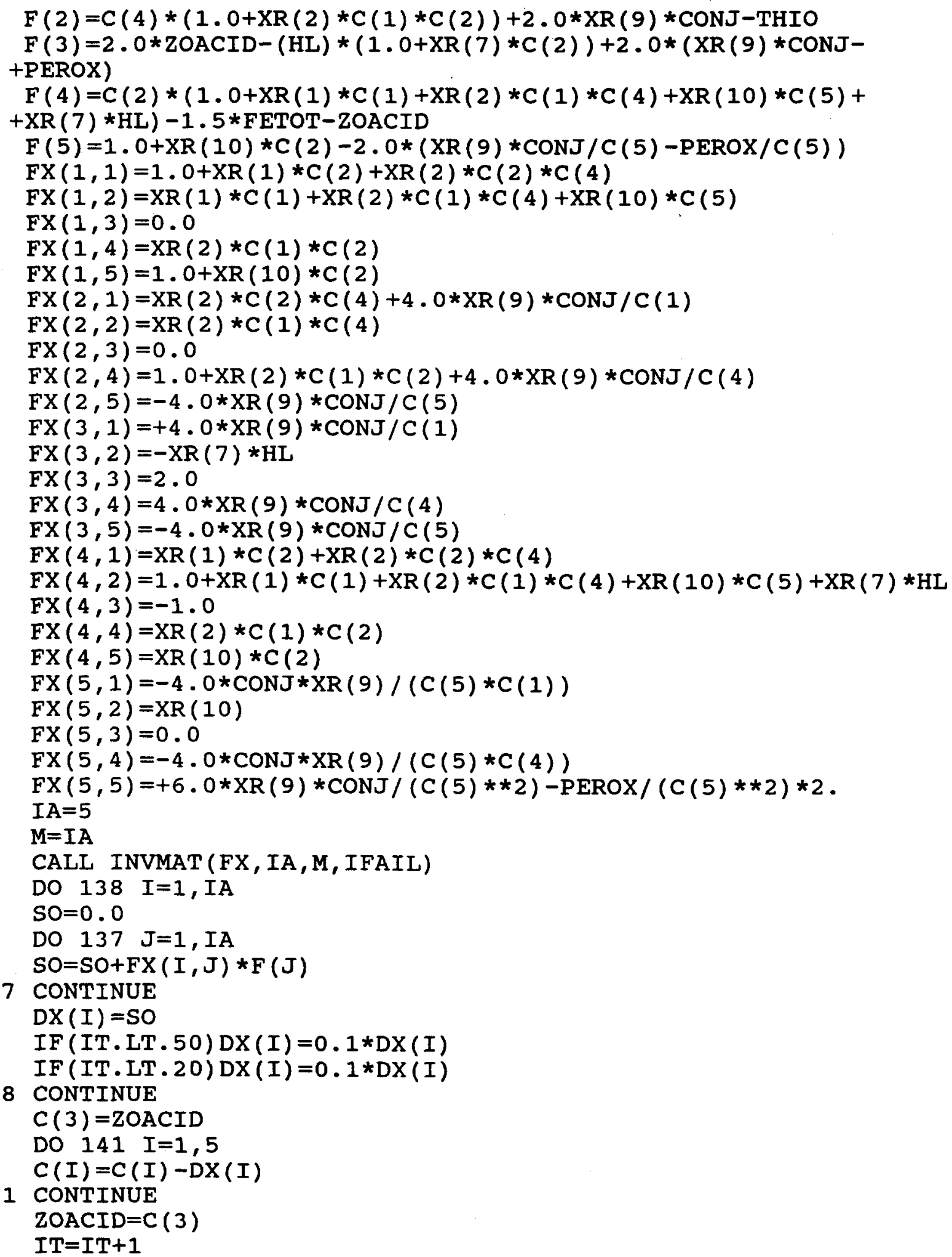




\title{
Apéndice C
}

\author{
$\operatorname{ALTO}=\operatorname{ABS}(\mathrm{DX}(1) / C(1))$ \\ DO $139 I=2,5$ \\ $\mathrm{IF}(\mathrm{ABS}(\mathrm{DX}(\mathrm{I}) / \mathrm{C}(\mathrm{I})) \cdot \mathrm{GT} \cdot \mathrm{ALTO}) . \mathrm{ALTO}=\mathrm{ABS}(\mathrm{DX}(\mathrm{I}) / \mathrm{C}(\mathrm{I}))$ \\ 139 CONTINUE \\ IF (ALTO.GT.1.0E-03) GO TO 136 \\ IF (C(4).LT.0.0) GO TO 300 \\ $I A=6 * N T+1$ \\ $\mathrm{NF}=17 * \mathrm{NT}+1$ \\ STOT $=$ FETOT $* 1.5+Z O A C I D$ \\ FADS $=X R(9) *$ CONJ \\ $\operatorname{READ}(17, *)$ CUREAC, BMCU \\ CONSUMO $=$ CUREAC $*$ BMCU * SOLIQ/63 . 54 \\ AOC $=$ CONSUMO $/$ AGOXID \\ WRITE $(18, *) C(3)$ \\ WRITE $(8,140) C(1), C(2), C(4), C(3)$, FADS , CONSUMO \\ 140 FORMAT (' ION FERRICO $=$, E10.3, $/$, \\ $+\prime$ SULFATO LIBRE $=$, E10.3,,$'$ TIOUREA LIBRE $=$, E10.3,, , \\ $+\prime$ TOTAL DE ACIDO ANADIDO $=$, E10.2, /,' FADS $=$, E10.3, /, \\ $+\prime$ OXIDANTE CONSUMIDO POR COBRE $=\prime$, E10.3, $/ /$, \\ $+{ }^{\prime} \quad$ PERFILES ADIMENSIONALES', /) \\ $\operatorname{IF}(C(1) . \operatorname{LE} .0 .0) C(1)=\operatorname{ABS}(C(1))$ \\ C \\ C LECTURA DE TIEMPO Y CONVERSION EXPERIMENTALES PARA AJUSTAR LA \\ C SIMULACION A TIEMPO REAL. \\ C \\ $\operatorname{READ}(17, *)$ TEXP , CONVEX \\ $I T C=0$ \\ IF (TEXP.EQ .0.0) ITC $=2$ \\ IF (ITC.EQ.2) WRITE $(8, *)$ 'SIN AJUSTE EXPERIMENTAL' \\ IF (ITC.NE.2) WRITE $(8, *)$ ' TIEMPO DE AJUSTE $=$ ', TEXP \\ IF (ITC.EQ.2) WRITE $(8, *)$ 'DIFUSIVIDAD EFECTIVA $=$ ', DEF \\ APROX $=C(1) / A G O X I D$ \\ $I T G=0$ \\ ITG2 $=0$ \\ $I S O L=0$ \\ ITSOL $=0$ \\ C \\ C VALORES INICIALES DE LOS PERFILES DE CONCENTRACION PARA EL \\ C PRIMER TIEMPO \\ C \\ 148 IF (ITSOL. EQ.1) ISOL $=1$ \\ $149 \operatorname{READ}(5, *)$ GUESS \\ $150 X(1)=C(1) /(C(1)+C(4) *$ GUESS $* 1 . E-9) * C(1) /$ AGOXID \\ $\mathrm{X}(\mathrm{NT}+1)=1.05 * \mathrm{C}(2) / \mathrm{AGOXID}$ \\ $\mathrm{X}(2 * \mathrm{NT}+1)=0.99 * \mathrm{HL} / \mathrm{AGOXID}$ \\ $X(3 * N T+1)=9.0 E-01 * C(4) / A G O X I D$ \\ IF (ISOL.NE. 1) GO TO 995 \\ $995 \times(4 * N T+1)=0.999 E-00 * C(5) / A G O X I D$ \\ $\mathrm{X}(5 * \mathrm{NT}+1)=\mathrm{X}(1) * \mathrm{X}(3 * \mathrm{NT}+1) * * 2 * \mathrm{XR}(12) * \mathrm{AGOXID} / \mathrm{X}(4 * \mathrm{NT}+1)$
}




\section{Apéndice $C$}

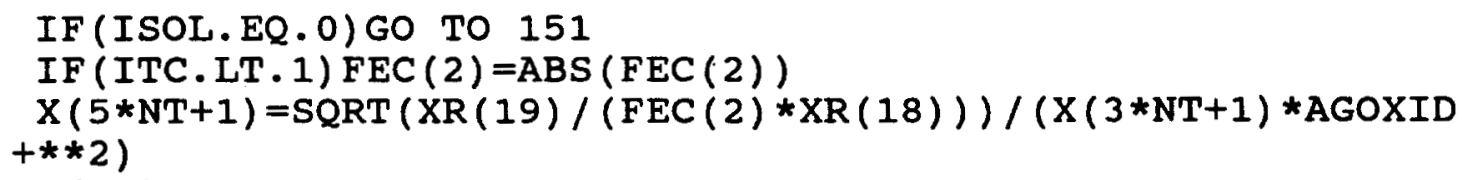

$151 \times(I A)=0.998$

$X(N T)=C(1) / A G O X I D$

$X(2 * N T)=C(2) /$ AGOXID

$\mathrm{X}(3 * \mathrm{NT})=\mathrm{HL} / \mathrm{AGOXID}$

$X(4 * N T)=C(4) / A G O X I D$

$X(5 * N T)=C(5) / A G O X I D$

$X(6 * N T)=5.0 \mathrm{E}-06$

$153 \mathrm{XO}(\mathrm{IA})=1.0$

$\mathrm{XO}(\mathrm{NT})=\mathrm{C}(1) / \mathrm{AGOXID}$

$\mathrm{XO}(2 * \mathrm{NT})=\mathrm{C}(2) / \mathrm{AGOXID}$

$\mathrm{XO}(3 * \mathrm{NT})=\mathrm{HL} / \mathrm{AGOXID}$

$\mathrm{XO}(4 * \mathrm{NT})=\mathrm{C}(4) /$ AGOXID

$\mathrm{XO}(5 * \mathrm{NT})=\mathrm{C}(5) / \mathrm{AGOXID}$

$\mathrm{XO}(6 * \mathrm{NT})=0.0$

DO $190 \mathrm{KA}=1, \mathrm{NT}-1$

$\mathrm{WD}=\mathrm{NT}-1$

$\mathrm{KB}=\mathrm{KA}+\mathrm{NT}$

$\mathrm{KC}=\mathrm{KB}+\mathrm{NT}$

$\mathrm{KD}=\mathrm{KC}+\mathrm{NT}$

$\mathrm{KE}=\mathrm{KD}+\mathrm{NT}$

$\mathrm{KF}=\mathrm{KE}+\mathrm{NT}$

$\mathrm{XO}(\mathrm{KA})=\mathrm{XO}(\mathrm{NT})$

$\mathrm{XO}(\mathrm{KB})=\mathrm{XO}(2 * \mathrm{NT})$

$\mathrm{XO}(\mathrm{KC})=\mathrm{XO}(3 * \mathrm{NT})$

$\mathrm{XO}(\mathrm{KD})=\mathrm{XO}(4 * \mathrm{NT})$

$\mathrm{XO}(\mathrm{KE})=\mathrm{XO}(5 * \mathrm{NT})$

$\mathrm{XO}(\mathrm{KF})=0.0$

DO $185 \mathrm{~J}=1,6$

$\mathrm{JJ}=\mathrm{J}-1$

$X(J J * N T+K A)=X(J J * N T+1)-E T A(K A) *(X(J J * N T+1)-X(J * N T))$

185 CONTINUE

190 CONTINUE

DO $191 \mathrm{~J}=1, \mathrm{IA}$

BOUND $(J)=1.0 E-25$

c

191 CONTINUE

c

PARAMETROS DE LAS ITERACIONES EN NEWTON-RAPHSON

$$
\begin{aligned}
& I A=6 * N T+1 \\
& N=I A \\
& J A G=0 \\
& I=0 \\
& T A U=0.0 \\
& S T=0.1 \\
& \text { ITCT }=0
\end{aligned}
$$




\section{Apéndice $C$}

IF (ITC.EQ. 2) WRITE $(8, *) '$ DIFUSUVIDAD EFECTIVA = ', DEF PHN $=-A L O G 10(X(3 * N T) * A G O X I D *$ SQRT $($ FAC $(8)) * 0.617)$

WRITE $(18, *)$ ' PH REAL $=$, , PHN

IF(ITC.EQ. 2) WRITE $(8, *) '$ PH REAL = ', PHN

C

C COMENZAR EL "LOOP" PARA CALCULAR LA CONVERSION Y TODAS LAS

C CONCENTRACIONES A CADA TIEMPO. SE AUMENTA EL VALOR DE DELTA

C TIEMPO APROXIMADAMENTE CADA CINCO TIEMPOS MEDIANTE EL VALOR

C DE ST.

C
DO $290 \mathrm{~L}=1,100$
IF (L.GT. 2) ST=1.0E00
IF (L.GT.5) ST=1 . OE01
IF (L.GT . 10) ST=3 . 0E01
IF (L.GT. 15) ST=1 . OE02
IF (L.GT . 20) ST=2 . OEO2
IF (L.GT. 25) ST=3 .0E02
IF (L.GT . 30) ST=6 . 0E02
IF (L.GT. 35) ST=1 . OE03
IF (L.GT.40) ST=3 . 0E03
IF (L.GT. 50) ST=6.0E03
IF (L.GT. 60) ST=1.0E04
IF (L.GT. 70) ST=2 . OE04
IF (L.GT. 80) ST=3 . OE04
IF (L.GT . 90) ST=4 . OE04
IF (L.GT . 100) ST $=5.0 E 04$
IF (L.GT.120) ST=6.0E04
$\mathrm{TAU}=\mathrm{TAU}+0.001 * \mathrm{ST}$

C

C A CADA TIEMPO SE ALIMENTAN LOS NUEVOS VALORES DE TIEMPO Y DE C LAS CONCENTRACIONES EN EL TIEMPO ANTERIOR.

C

$\operatorname{PAR}(2)=\mathrm{TAU}$

$\operatorname{PAR}(3)=I S O L$

$\operatorname{PAR}(4)=\mathrm{L}$

NSIG $=5$

ITMAX $=250$

DO 206 IASIGN $=1$, IA

$\operatorname{PAR}(4+\operatorname{IASIGN})=\mathrm{XO}(\mathrm{IASIGN})$

206 CONTINUE

c

207 CONTINUE

C SI LAS CONCENTRACIONES LLEGAN AL LIMITE DE SOLUBILIDAD EN LA

C FRONTERA DEL NUCLEO, SE REASIGNAN PERFILES (ITSOL = 1)

C

208 IF (ITSOL. GE . 1) TAU $=$ TAU $-0.001 *$ ST

IF (ITSOL. GE. 1) ST $=0.50 * S T$

IF (ITSOL. GE. 1) TAU $=$ TAU $+0.001 * \mathrm{ST}$

$\operatorname{PAR}(1)=S T$ 
Apéndice C

C

C

209 CALL ZSPOW (FCN, NSIG, N, ITMAX, PAR, X, FNORM, WK, IER)

IF (IER. GT . 128) WRITE (18, *) IER, I

IF (IER.GT . 128) WRITE ( 8, *) IER, L

IF (IER . GT . 128) ITCT=ITCT+1

IF (ITCT.GT. 10) GO TO 310

IF(ITC .LT.2) GO TO 263

259 CONTINUE

260 IF (X(IA).LT.0.14) GO TO 280

IF (X(IA) . GE.1.01) GO TO 310

C

C CALCULO DE LAS CONCENTRACIONES DE LAS ESPECIES DE PLATA Y DEL

C ION FERRICO A PARTIR DE LAS DE LOS IONES LIBRES. C

$\operatorname{FEC}(1)=\mathrm{XR}(1) * \mathrm{X}(1) * \mathrm{X}(\mathrm{NT}+1) * \mathrm{AGOXID} * * 2$

$\operatorname{FEC}(2)=X R(2) * X(1) * X(N T+1) * X(3 * N T+1) *$ AGOXID $* * 3$

AGL $=X R(13) * X(5 * N T+1) /((X(3 * N T+1) * * 2) * A G O X I D)$

$\operatorname{AGT} 1=X R(14) * X(5 * N T+1) / X(3 * N T+1)$

$\mathrm{AGT} 2=\mathrm{X}(5 * \mathrm{NT}+1) * \mathrm{AGOXID}$

$\mathrm{AGT} 3=\mathrm{XR}(15) * \mathrm{X}(5 * \mathrm{NT}+1) * \mathrm{X}(3 * \mathrm{NT}+1) * \mathrm{AGOXID} * * 2$

AGT $4=X R(16) * X(5 * N T+1) * X(3 * N T+1) * * 2 * A G O X I D * * 3$

AG2T $3=X R(17) * X(5 * N T+1) * * 2 * \operatorname{AGOXID} / X(3 * N T+1)$

$\mathrm{AG} 2 \mathrm{~T} 6=\mathrm{XR}(18) *(\mathrm{X}(5 * \mathrm{NT}+1) * \mathrm{X}(3 * \mathrm{NT}+1)) * * 2 * \mathrm{AGOXID} * 4$

AGLS $=X R(13) * X(6 * N T) \quad /((X(4 * N T) * * 2) * A G O X I D)$

AGT1S $=X R(14) * X(6 * N T), / X(4 * N T)$

AGT2S $=X(6 * N T) * A G O X I D$

$\mathrm{AGT} 3 \mathrm{~S}=\mathrm{XR}(15) * \mathrm{X}(6 * \mathrm{NT}) * \mathrm{X}(4 * \mathrm{NT}) \quad$ *AGOXID**2

$\mathrm{AGT} 4 \mathrm{~S}=\mathrm{XR}(16) * \mathrm{X}(6 * \mathrm{NT}) * \mathrm{X}(4 * \mathrm{NT}) * * 2 \quad$ *AGOXID $* * 3$

$A G 2 T 3 S=X R(17) * X(6 * N T) * * 2 * A G O X I D / X(4 * N T)$

AG2T6S $=X R(18) *(X(6 * N T) * X(4 * N T)) * * 2 * A G O X I D * * 4$

AGSUL $=X R(19) / F E C(2)$

IF ( AG2T6. GE. AGSUL) ISOL $=1$

IF (ISOL. EQ . 1) ITSOL=ITSOL+1

C

IF (ITSOL. EQ.1) WRITE $(18, *)$ L

C

VERIFICA SI HAYA LLEGADO AL LIMITE DE SOLUBILIDAD.

IF (ITSOL. EQ. 1)WRITE $(8, *) ' A g 2 T 6+2=\prime, A G 2 T 6$,

$+\prime$ LIMITE $=$ ', AGSUL

IF (ITSOL.NE.1) GO TO 263

IF (ISOL.EQ.1) WRITE( $8, *)^{\prime}$ PRECIPITACION DE Ag2SO4.3Tu.H2O'

C

263 IF(ITSOL.EQ.1)GO TO 148

C CALCULO DEL TIEMPO REAL, LA CONVERSION Y EL PH NUEVO.

TIME $=$ TAU $*$ RADPAR * *2 $/$ DEF $/ 60$.

$\mathrm{CONV}=1.0-\mathrm{X}(\mathrm{IA}) * * 3$ 
Apéndice $C$

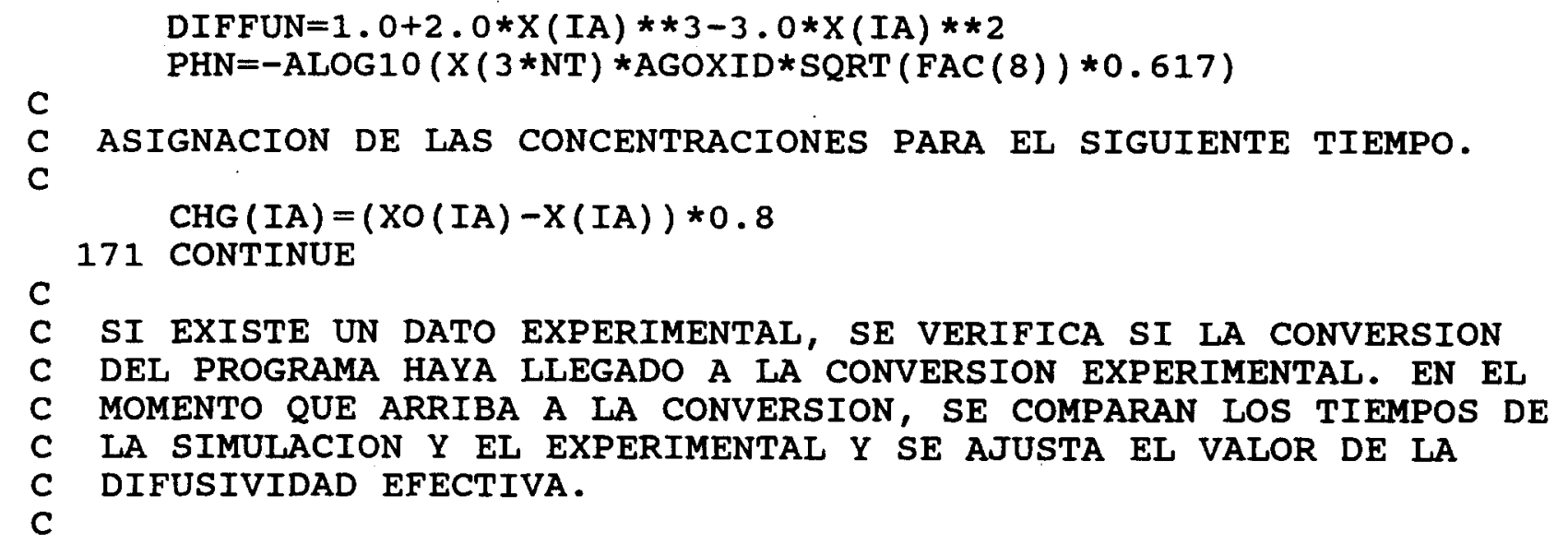

IF (ITC.EQ.2) GO TO 264

IF (CONV. LT. CONVEX) GO TO 264

CONVO $=1.0-\mathrm{XO}(\mathrm{IA}) * \star 3$

TAU5O $=($ TAU $-S T * 0.001 *(1.0-($ CONVEX - CONVO $) /($ CONV - CONVO $)))$

TIME50 $=$ TAU 50*RADPAR * $* 2 /(\mathrm{DEF} * 60$.

$\mathrm{DEF}=\mathrm{TIME} 50 / \mathrm{TEXP} * \mathrm{DEF}$

$I T C=I T C+1$

GO TO 150

C

264 DO $265 I=1, I A$

C SE VERIFICA QUE NINGUNA CONCENTRACION SEA MENOR QUE CERO.

C DE LO CONTRARIO SE REGRESA A LA ASIGNACION DE VALORES DE LAS

C CONCENTRACIONES (150) PARA REASIGNAR NUEVOS PERFILES.

C

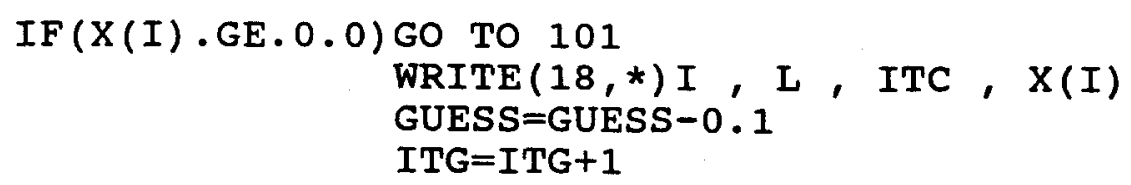

IF ( I . GT . 20) GUESS=GUESS +0.15

WRITE $(18, *)$ GUESS

IF (ITG.GT.50) GO TO 310

GO TO 150

$101 \mathrm{XO}(\mathrm{I})=\mathrm{X}(\mathrm{I})$

265 CONTINUE

$X(I A)=X(I A)-C H G(I A)$

IF (ITC.NE. 2) GO TO 290

IF (CONV.LT.0.10) GO TO 169

IF (CONV.GT.0.15) GO TO 163

C

C SE CAPTAN TRES VALORES ESPACIADOS DE CONVERSION VERSUS

C TIEMPO PARA MAS ADELANTE AJUSTAR UNA FUNCION DE CONVERSION

C
$\operatorname{AJX}(1)=\mathrm{CONV}$
$\operatorname{AJT}(1)=$ TIME
GO TO 169 
Apéndice C

163 IF (CONV.LT.0.40) GO TO 169

IF (CONV.GT.0.45) GO TO 165

$\operatorname{AJX}(2)=\mathrm{CONV}$

$\operatorname{AJT}(2)=T I M E$

GO TO 169

165 IF (CONV.LT.0.75) GO TO 169

IF (CONV.GT.0.80) GO TO 169

$\operatorname{AJX}(3)=\mathrm{CONV}$

$\operatorname{AJT}(3)=T I M E$

$169 \mathrm{FL}=\mathrm{L}$

$F R A C=F L / 5.0$

IFRAC $=$ INT $($ FRAC $)$

IF (L.EQ.1) GO TO 280

C

C CALCULAR LA CANTIDAD DE OXIDANTE CONSUMIDO POR LA LIXIVIACION

C Y SI ESTA AGOTANDO, SALIR DEL PROGRAMA.

C

C

$\mathrm{X}(28)=\mathrm{XR}(1) * \mathrm{AGOXID} * \mathrm{X}(4) * \mathrm{X}(8)$

$\mathrm{X}(32)=\mathrm{XR}(2) * \mathrm{AGOXID} * * 2 * \mathrm{X}(4) * \mathrm{X}(8) * \mathrm{X}(16)$

$A G O X R=2.0 * X(40)+X(4)+X(28)+X(32)$

IF (AGOXR . LT . 1 . OE-03) JAG $=1$

IF (JAG.EQ.1) WRITE $(8, *)$ 'AGOTAMIENTO DE OXIDANTE'

IF (JAG.EQ.1) AJX (3) =CONV

IF (JAG.EQ.1) AJT (3)=TIME

IF (JAG.EQ.1) GO TO 310

C

IMPRIMIR PERFILES

IF (L.EQ.20) GO TO 280

IF (L.EQ.40) GO TO 280

IF (L.EQ.60) GO TO 280

IF (CONV.LT.0.01) GO TO 290

C

266 IF (CONV.GT.0.10) GO TO 267

C IMPRIMIR RESULTADOS

C

IF (ABS (IFRAC-FRAC) . GT.0.0) GO TO 290

267 WRITE $(8,270)$ TAU, X (IA), TIME, CONV, DIFFUN, PHN

270 FORMAT (F9.3, 2X, 4 (E11.3,2X), 3X, F5.2)

GO TO 290

280 DO $283 \mathrm{~J}=1,6$

WRITE $(8,282)(X((J-1) * N T+K A), K A=1, N T)$

282 FORMAT ( 4 E10.3)

283 CONTINUE

WRITE ( 8,285 ) AGL , AGT1, AGT2 , AGT3 , AGT4, AG2T3, AG2T6, AGLS, AGT1S, + AGT2S, AGT3S, AGT4S, AG2T3S, AG2T6S, FEC (2)

285 FORMAT (/, 17X, 'AG+', 5X,'AGT+', 5X,'AGT2+', 4X, 'AGT3+', 4X, +'AGT4+', $2 \mathrm{X}$, 'AG2T3+2',2X,'AG2T6+2', /, 'EN LA SUPER',

+6E9.2, E9.3,1,'EN SOLUCION ',6E9.2, E9.3,1,' FesO4Tu = ', 
Apéndice C

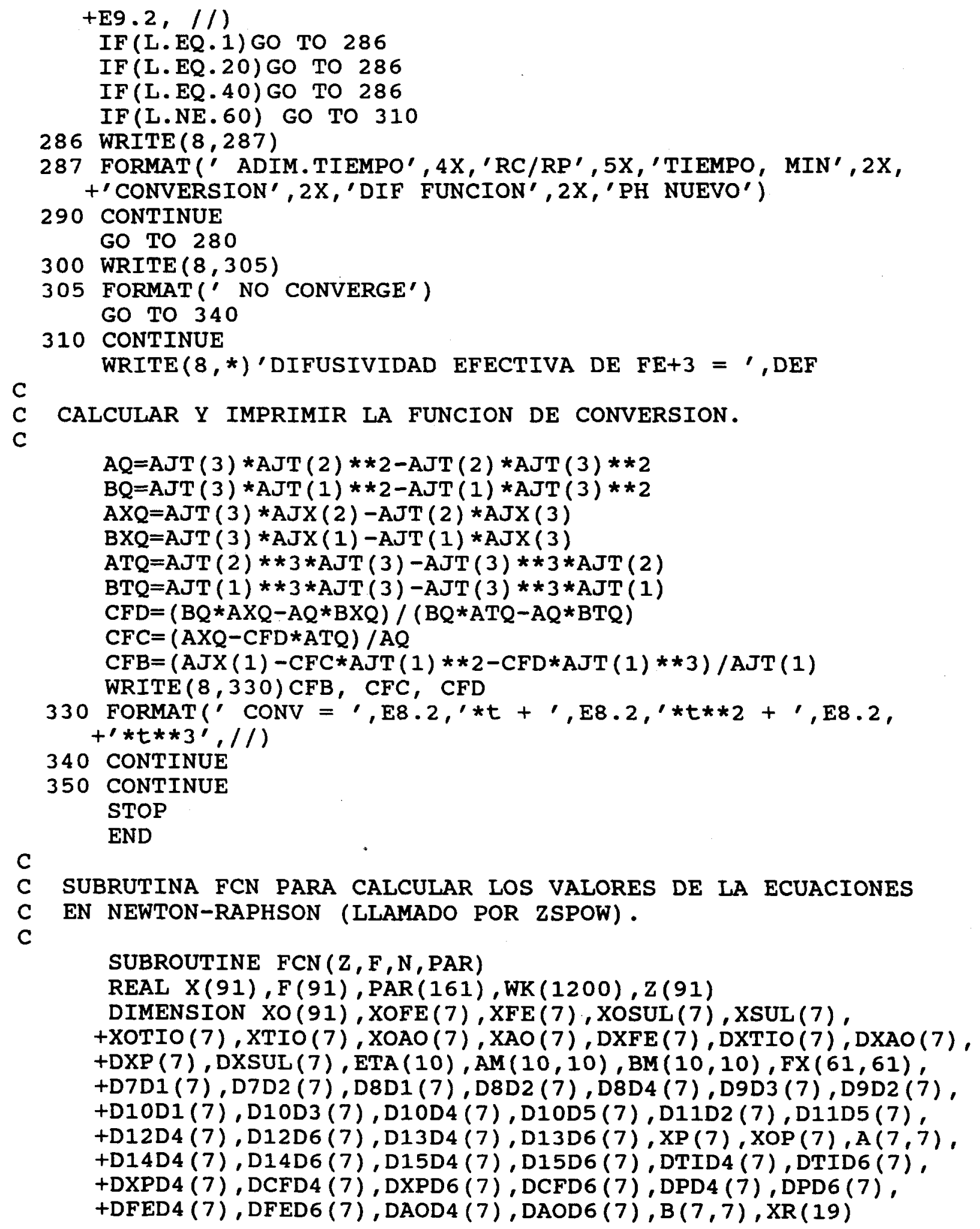


COMMON / DIFU / DELB , DELC , DELD , DELE , DELF , DELG , DELH , DELI , +DELJ , DELK , DELL , DELM , DELN , DELP , DELO , DELQ , DELR COMMON/SYST/G, AGOXID, COX, FETOT, STOT , THIO , AS , SOLIQ , GPTAG ,+ EPT, RADPAR, DEF , PEROX COMMON/NPUNTOS/NT , AM, BM , ETA, IA, NF COMMON / CONEQ/XR

DO $3 \mathrm{~J}=1$, IA

$X(\mathrm{~J})=\mathrm{Z}(\mathrm{J})$

3 CONTINUE

$X(N F)=X(I A)$

$X R O N E=X R(1)$

$X R T W O=X R(2)$

$\mathrm{XRSVN}=\mathrm{XR}(7)$

$X R N I N=X R(9)$

$X R T E N=X R(10)$

$X R E L E=X R(11)$

$X R E Q=X R(12)$

$\mathrm{XRTHIR}=\mathrm{XR}(13)$

$X R F R T N=X R(14)$

$X R F I F=X R(15)$

$\mathrm{XRSXTN}=\mathrm{XR}(16)$

XRSVTN=XR (17)

$X R E I T N=X R(18)$

$\mathrm{XRSP}=\mathrm{XR}(19)$

TIO $=$ THIO

$S T=P A R(1)$

TAU $=$ PAR (2)

ISOL $=$ PAR ( 3 )

$\mathrm{L}=\mathrm{PAR}(4)$

DO 85 IASIGN $=1$, IA-1

$\mathrm{XO}(\mathrm{IASIGN})=\mathrm{PAR}(4+\mathrm{IASIGN})$

85 CONTINUE

$\mathrm{XO}(\mathrm{NF})=\operatorname{PAR}(4+\mathrm{IA})$

C

C CALCULAR LAS CONCENTRACIONES

C EQUILIBRIO CON LAS VARIABLES

DE LAS 11 ESPECIES COMPLEJOS EN

C COLOCACION Y LAS FRONTERAS

c
DO $100 \mathrm{KA}=1$, NT
$\mathrm{KB}=\mathrm{KA}+\mathrm{NT}$
$\mathrm{KC}=\mathrm{KB}+\mathrm{NT}$
$\mathrm{KD}=\mathrm{KC}+\mathrm{NT}$
$\mathrm{KE}=\mathrm{KD}+\mathrm{NT}$
$\mathrm{KF}=\mathrm{KE}+\mathrm{NT}$
$\mathrm{KG}=\mathrm{KF}+\mathrm{NT}$
$\mathrm{KH}=\mathrm{KG}+\mathrm{NT}$
$\mathrm{KI}=\mathrm{KH}+\mathrm{NT}$
$\mathrm{KJ}=\mathrm{KI}+\mathrm{NT}$
$\mathrm{KK}=\mathrm{KJ}+\mathrm{NT}$ 
Apéndice C

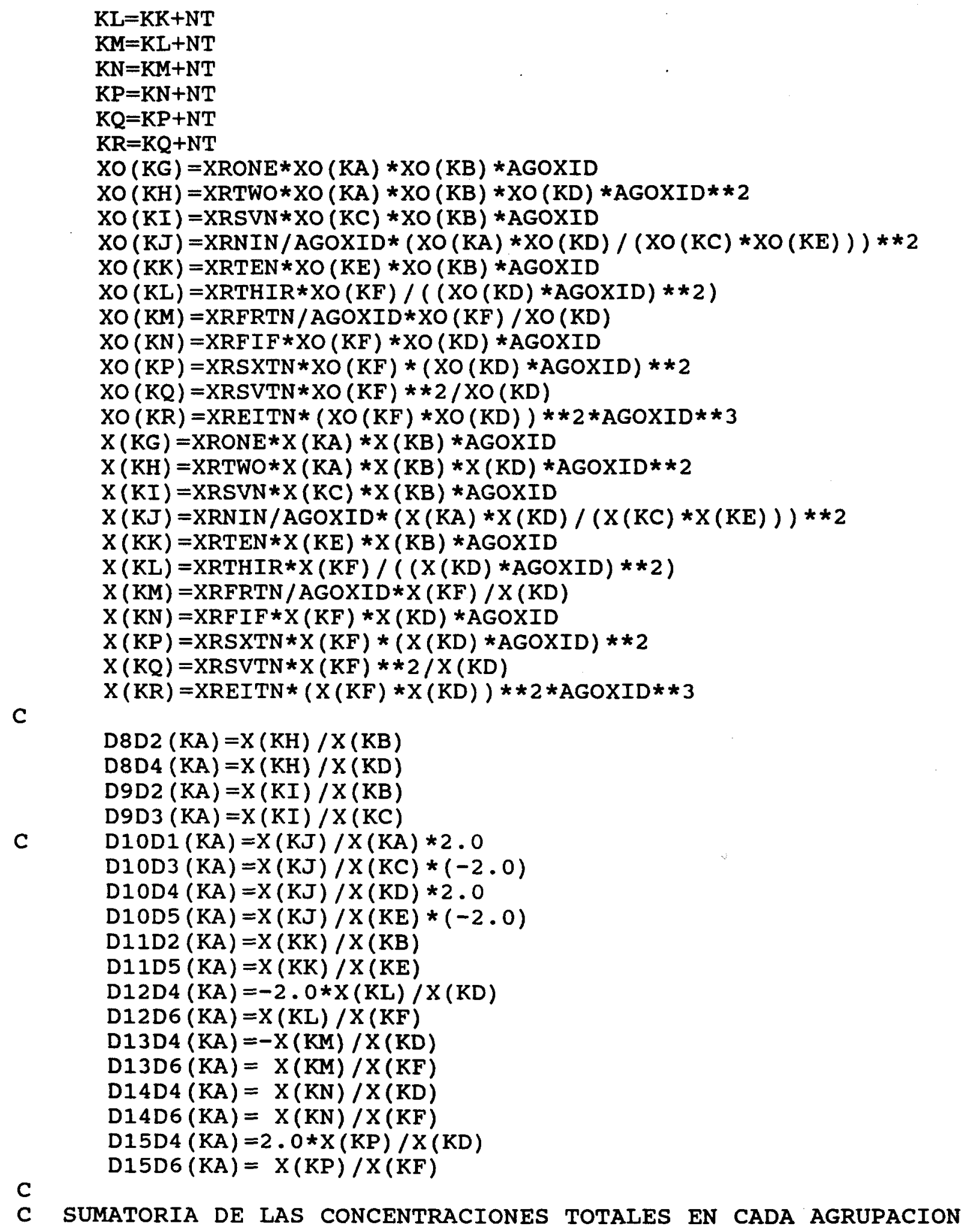


Apéndice C

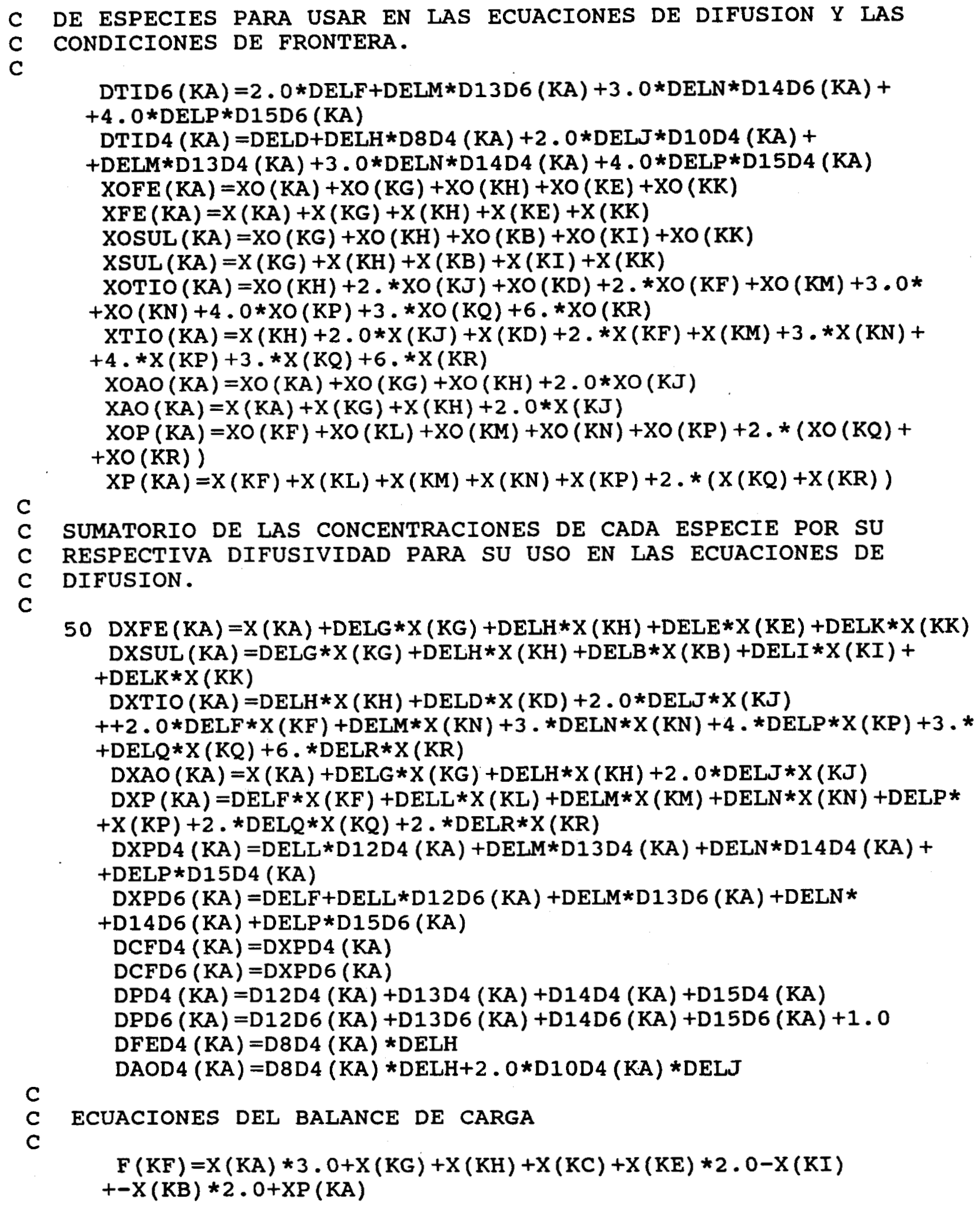


Apéndice C

C

100 CONTINUE

C ECUACIONES PARA LA CONDICION DE FRONTERA EN RC (FLUXES)

$\mathrm{C}$

$F(1)=0.0$

$\mathrm{F}(1+\mathrm{NT})=0.0$

$F(1+2 * N T)=0.0$

$F(1+3 * N T)=0.0$

DO $200 \mathrm{~J}=1, \mathrm{NT}$

$\mathrm{F}(1)=\mathrm{F}(1)+\mathrm{AM}(1, \mathrm{~J}) \star \mathrm{DXFE}(\mathrm{J})$

$\mathrm{F}(1+\mathrm{NT})=\mathrm{F}(1+\mathrm{NT})+\mathrm{AM}(1, \mathrm{~J}) * \mathrm{DXSUL}(\mathrm{J})$

$\mathrm{F}(1+2 * \mathrm{NT})=\mathrm{F}(1+2 * \mathrm{NT})+\mathrm{AM}(1, \mathrm{~J}) *(\mathrm{DXTIO}(\mathrm{J}))$

200 CONTINUE

$F(1+3 * N T)=F(1+3 * N T)+A M(1, J) *(D X A O(J)+D X P(J))$

IF (ISOL.EQ.1) GO TO 202

$\mathrm{EQEQ}=(\mathrm{ABS}(\mathrm{X}(4 * \mathrm{NT}+1) * \mathrm{X}(5 * \mathrm{NT}+1) /(\mathrm{X}(1) * \mathrm{X}(3 * \mathrm{NT}+1) * * 2 *$

+ AGOXID*XREQ) ) $) * * 0.5$

$\mathrm{F}(1+4 * \mathrm{NT})=\mathrm{EQEQ}-1.0$

GO TO 205

C

C SI EL SISTEMA HA LLEGADO AL LIMITE DE SOLUBILIDAD, ENTONCES

C SE SUSTITUYE LA ECUACION DE EQUILIBRIO [F(1+4*NT)] POR EL

C PRODUCTO DE SOLUBILIDAD.

C

202 EQSOL $=X(16 * N T+1) * X(7 * N T+1) * A G O X I D * * 2 / X R S P$

$\mathrm{F}(4 * \mathrm{NT}+1)=\mathrm{ABS}(\mathrm{EQSOL}) * * .5-1.0$

C

C ECUACIONES PARA LA CONDICION DE FRONTERA EN RP (BALANCES DE

C MATERIA).

C

$205 \mathrm{~F}(\mathrm{NT})=\mathrm{XFE}(\mathrm{NT})-\mathrm{FETOT} / \mathrm{AGOXID}$

$F(2 * N T)=X S U L(N T)-S T O T / A G O X I D$

$\mathrm{F}(3 * \mathrm{NT})=\mathrm{XTIO}(\mathrm{NT})-\mathrm{TIO} * \mathrm{EXP}(-\mathrm{AS} * \mathrm{RADPAR} * 2 /(\mathrm{DEF} * 60$.$) *$

+ TAU) / AGOXID

$\mathrm{F}(4 * \mathrm{NT})=\mathrm{XAO}(\mathrm{NT})+\mathrm{XP}(\mathrm{NT})-1.0$

$\mathrm{NF}=\mathrm{NT} * 17+1$

$F(5 * N T)=X P(N T)-G P T A G * 9.28 E-06 *$ SOLIQ* $(1.0-X(N F) * * 3) /$

+ AGOXID

FIS $=1.0 /(1.0-X(N F)) * * 2$

DFIS $=2.0 * F I S /(1.0-X(N F))$

$\mathrm{CF}=0.0$

DO $210 \mathrm{~J}=1, \mathrm{NT}$

$\mathrm{CF}=\mathrm{CF}+\mathrm{AM}(1, \mathrm{~J}) * \mathrm{DXP}(\mathrm{J})$

210 CONTINUE

$\mathrm{U}=0.001 * \mathrm{ST} * \mathrm{FIS} / \mathrm{EPT}$

$\mathrm{NA}=6 * \mathrm{NT}+1$ 


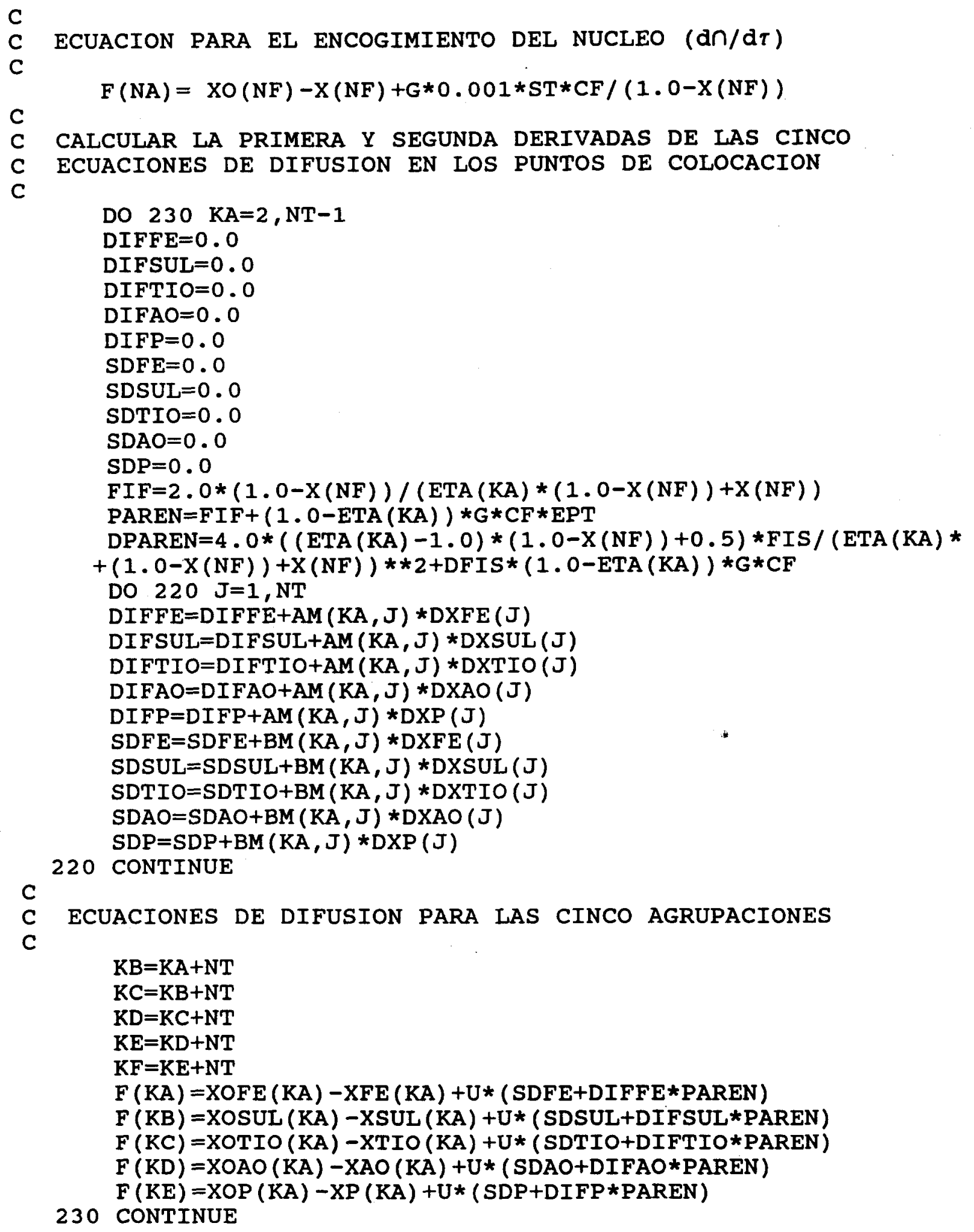




\title{
Apéndice C
}

\author{
250 CONTINUE \\ $\mathrm{X}(\mathrm{IA})=\mathrm{X}(\mathrm{NF})$ \\ DO $290 \mathrm{~J}=1$, IA \\ $\mathrm{Z}(\mathrm{J})=\mathrm{X}(\mathrm{J})$ \\ 290 CONTINUE \\ RETURN \\ END
}


Apéndice C

\section{C.2. - METODOS NUMERICOS UTILIZADOS EN EL PROGRAMA}

Para escoger los métodos númericos a utilizar, habría que observar los problemas que están implicitos en este sistema de ecuaciones. En primer lugar, para fijar la frontera móvil del núcleo se transforma la variable radial, resultando en un dominio que no existe a $t=0$ (se presenta una singularidad). En segundo, la condición de frontera de equilibrio, ecuación (45), introduce un aspecto no-lineal al problema. El tercer problema que se encuentra es que, a tiempos muy pequeños, las concentraciones de las especies cambian bruscamente del valor inicial para luego estabilizarse, mientras la posición del núcleo tiene una tendencia opuesta. Finalmente, para considerar diecisiete especies en solución por separado, relacionadas a través de las reacciones a equilibrio, resulta muy dificil proponer perfiles iniciales de concentración para cada especie. Por eso, se tiene que reducir el número de variables $\mathrm{y}$ ecuaciones a seis especies $\left(\mathrm{Fe}^{3+}, \mathrm{SO}_{4}{ }^{2-}, \mathrm{H}^{+}, \mathrm{CS}\left(\mathrm{NH}_{2}\right)_{2}, \mathrm{Fe}^{2+}\right.$ Y Ag[CS $\left.\left.\left(\mathrm{NH}_{2}\right)_{2}\right]_{2}{ }^{+}\right)$, incorporando la definición de las especies complejas (sus respectivas reacciones de complejación) a las ecuaciones de difusión, situación que añade más no-linealidad al sistema $y$ lo vuelve mal condicionado debido a que las concentraciones de los iones libres son diferentes en hasta once ordenes de magnitud.

Por lo anterior, se optó por el método de colocación ortogonal con polinomios desplazados de Legendre (con la potencias de ponderación, alfa y beta, = 0) para discretizar la parte radial de las ecuaciones de difusión (5) y las de densidad de flujo en las condiciones de frontera $Y$ en la ecuación para describir el encogimiento del núcleo. Debido a la suavidad de los perfiles de concentración, no hay una mejora sustancial a la solución númerica con más de dos puntos de colocación. Para generar las matrices [ $A_{i j}$ ] 


\section{Apéndice C}

$Y\left[B_{i j}\right]$, se emplean las subrutinas COEFF, JCOBI, INTRP Y SIMP del libro de Villadsen y Michelsen (1978).

Con respecto a la parte temporal de las ecuaciones de difusión $y$ de encogimiento del núcleo, se discretiza el operador diferencial utilizando el método de Euler implícito para evitar la singularidad a tiempo cero, incrementando el valor de $\Delta t$ conforme aumenta el tiempo.

El sistema de ecuaciones diferenciales discretizadas junto con los balances de materia y de carga, se resuelven para cada tiempo por el método de Newton-Raphson mediante la subrutina zsPow de la biblioteca IMSL.

Uno de las criticas dificultades que se enfrenta en este tipo de problema, precisamente por lo mal condicionado del sistema de ecuaciones, es su alta sensibilidad a los valores iniciales supuestos de concentración en el primer tiempo. Para ayudar a estimarlos, se realiza un cálculo por el método de Newton-Raphson en el seno de la solución a tiempo cero para las diez especies que se encuentran presentes antes de empezar la lixiviación. Posteriormente, los valores que resultan del cálculo anterior son usados para estimar las concentraciones en el seno de la solución al primer tiempo. La concentración de tiourea y las de los iones sulfato, ferroso e hidronio pueden suponerse constantes en todo el dominio por encontrarse en exceso. Entonces, la dificultad se reduce a fijar un valor para la especie $\mathrm{Fe}^{3+}$ en la frontera de $\mathrm{R}_{\mathrm{c}}$ ( $\mathrm{n}$ $=0)$ porque el valor de la especie $\mathrm{Ag}\left[\mathrm{CS}\left(\mathrm{NH}_{2}\right)_{2}\right]_{2}{ }^{+}$en este punto está dado por la ecuación de equilibrio de la reacción de oxidación, la ecuación (45). Teniendo ambos valores en las fronteras para las dos especies cambiantes, se supone perfiles lineales. A los siguientes tiempos, se utilizan aquellas concentraciones que resultaron de la 
Apéndice $C$

iteración de Newton-Raphson para el tiempo anterior como valores iniciales de concentración para el tiempo que se está considerando.

\section{C.3. - CONSTANTES DE EQUILIBRIO Y SU CORRECCION PARA LA FUERZA IONICA}

La mayoría de las constantes de equilibrio están reportados en los libros de Smith y Martell (1976 y 1982). En el caso del complejo $\mathrm{FeSO}_{4}\left[\mathrm{CS}\left(\mathrm{NH}_{2}\right)_{2}\right]^{+}$, esta está determinada por Groenewald (1976). La siguiente tabla contiene los valores para las reacciones correspondientes a $25^{\circ} \mathrm{C}$ y una fuerza iónica de 1.0:

REACCION

$\mathrm{Fe}^{3+}+\mathrm{SO}_{4}^{2-} \Longleftrightarrow \mathrm{FeSO}_{4}^{+}$

$\mathrm{Fe}^{3+}+\mathrm{SO}_{4}^{2-}+\mathrm{CS}\left(\mathrm{NH}_{2}\right)_{2} \Longleftrightarrow \mathrm{FeSO}_{4} \mathrm{CS}\left(\mathrm{NH}_{2}\right)_{2}^{+}$

$\mathrm{H}^{+}+\mathrm{SO}_{4}{ }^{2-} \longleftrightarrow \mathrm{HSO}_{4}^{-}$

$\mathrm{H}^{2} \mathrm{O} \rightleftharpoons \mathrm{H}^{+}+\mathrm{OH}^{-}$

$2 \mathrm{Fe}^{3+}+2 \mathrm{CS}\left(\mathrm{NH}_{2}\right)_{2} \Longleftrightarrow 2 \mathrm{Fe}^{2+}+2 \mathrm{H}^{+}+\left(\mathrm{NH}_{2}\right) \mathrm{NHCS}-\mathrm{SCNH}\left(\mathrm{NH}_{2}\right)$

$\mathrm{Fe}^{2+}+\mathrm{SO}_{4}^{2-} \Longleftrightarrow \mathrm{FeSO}_{4}$

$\mathrm{Ag}^{+}+\mathrm{CS}\left(\mathrm{NH}_{2}\right)_{2} \Longleftrightarrow \mathrm{AgCS}\left(\mathrm{NH}_{2}\right)_{2}^{+}$

$\mathrm{Ag}^{+}+2 \mathrm{CS}\left(\mathrm{NH}_{2}\right)_{2} \Longleftrightarrow \mathrm{Ag}\left[\mathrm{CS}\left(\mathrm{NH}_{2}\right)_{2}\right]_{2}^{+}$

$\mathrm{Ag}^{+}+3 \mathrm{CS}\left(\mathrm{NH}_{2}\right)_{2} \Longleftrightarrow \mathrm{Ag}\left[\mathrm{CS}\left(\mathrm{NH}_{2}\right)_{2}\right]_{3}^{+}$

$\mathrm{Ag}^{+}+4 \mathrm{CS}\left(\mathrm{NH}_{2}\right)_{2} \Longleftrightarrow \mathrm{Ag}\left[\mathrm{CS}\left(\mathrm{NH}_{2}\right)_{2}\right]_{4}^{+}$
LOG $\mathrm{K}$

2.02

6.64

1.10

$-13.79$

11.84

2.20

7.11

10.61

12.73

13.57

Existe evidencia en la literatura más reciente que la complejación de tiourea con plata no es tan sencilla (Pesic y Seal, 1990; Gornostaeva et al., 1986; Khmel'nitskaya et al., 1985). Estos autores reportan la presencia de polímerización del complejo $\mathrm{Ag}\left[\mathrm{CS}\left(\mathrm{NH}_{2}\right)_{2}\right]_{3}{ }^{+}$y la precipitación de los sólidos $\mathrm{Ag}_{2}\left[\mathrm{CS}\left(\mathrm{NH}_{2}\right)_{2}\right]_{3} \mathrm{SO}_{4}\left(\mathrm{p}_{1}\right)$ y $\mathrm{Ag}_{2}\left[\mathrm{CS}\left(\mathrm{NH}_{2}\right)_{2}\right]_{6} \mathrm{SO}_{4}\left(\mathrm{p}_{2}\right)$; el primero sobre la superficie del disco rotante a las condiciones de lixiviación (Pesic y Seal, 1990). Sin 
Apéndice C

embargo, en ninguno de los trabajos citados calculan un producto de solubilidad de dichos sólidos..

Como la precipitación de estos sólidos son probablemente la causa del comportamiento anómalo de la lixiviación, tanto de la argentita como de la plata metálica a $0.3 \mathrm{M}$ de tiourea, es importante estimar, a partir de los datos electroquimicos presentados por Khmel'nitskaya et al. (1985), la constante de complejación de por lo menos uno de los precursores. En primer lugar, estos datos demuestran que en todo el intervalo de concentración de tiourea hasta $1.0 \mathrm{M}$ y baja concentración de plata, el complejo dominante de plata es el $\mathrm{Ag}\left[\mathrm{CS}\left(\mathrm{NH}_{2}\right)_{2}\right]_{3}{ }^{+}$. Esto contradice el valor reportado por Smith y Martell (1976) para la constante de estabilidad del complejo $\mathrm{Ag}\left[\mathrm{CS}\left(\mathrm{NH}_{2}\right)_{2}\right]_{4}{ }^{+}$, que resulta demasiado alto en, por lo menos, un orden de magnitud. Por la elevada precisión del método empleado por Khmel'nitskaya et al. (1985), se decidió reducir la constante del cuarto complejo con tiourea un orden de magnitud con respecto a aquel valor que aparece en Smith y Martell (1976). También en el mismo artículo de Khmel'nitskaya et al. (1985), existe evidencia de que, a más altas concentraciones de plata, el mismo tercer complejo dominante se polimeriza. A partir de los datos asi reportados se calculó una constante de complejación del dímero, $\mathrm{Ag}_{2}\left[\mathrm{CS}\left(\mathrm{NH}_{2}\right)_{2}\right]_{6}{ }^{2+}$, de $4 \times 10^{28}$. Cabe destacar que este cálculo es válido únicamente para los compuestos dominantes o presentes en una concentración cercana a éstos.

Con respecto al precursor, $\mathrm{Ag}_{2}\left[\mathrm{CS}\left(\mathrm{NH}_{2}\right)_{2}\right]_{3}{ }^{2+}$, del sólido p1, su existencia es probable, aunque no sea como complejo dominante por la siguiente evidencia (Pesic y Seal, 1990; Plaksin y Kozhukhova, 1960): la velocidad de lixiviación, tanto de $\mathrm{Ag}^{\circ}$ como de $\mathrm{Ag}_{2} \mathrm{~S}$, presenta un máximo alrededor de $0.1 \mathrm{M}$ de tiourea; ésta baja con un aumento en la concentración y luego vuelve a subir a 1.0M. Esto 
Apéndice C

significa que la concentración de uno de los precursores probablemente presenta un máximo a una concentración alrededor de $0.3 \mathrm{M}$ de tiourea $y$, consecuentemente, causa la precipitación. Sin la presencia del $\mathrm{Ag}_{2}\left[\mathrm{CS}\left(\mathrm{NH}_{2}\right)_{2}\right]_{3}{ }^{2+}$, ninguno de los dos precursores presenta un máximo a $0.3 \mathrm{M}$. En el caso de una constante de complejación de $\mathrm{Ag}_{2}\left[\mathrm{CS}\left(\mathrm{NH}_{2}\right)_{2}\right]_{3}{ }^{2+}$ alrededor de $10^{25}$, se cumple que la concentración máxima de $\mathrm{Ag}_{2}\left[\mathrm{CS}\left(\mathrm{NH}_{2}\right)_{2}\right]_{6}{ }^{2+}$ sea alrededor de $0.3 \mathrm{M}$ de tiourea sin que éste sea un complejo dominante.

La corrección a las constantes de equilibrio por la fuerza iónica de la solución, se llevó a cabo solamente al inicio del programa para las condiciones en el seno de ésta. Esta simplificación se justifica dado que los principales contribuyentes a la fuerza iónica, el ión sulfato y el ión hidronio libres, se mantienen esencialmente constantes en todo el transcurso de la lixiviación y a todo lo largo de la capa de inertes. Para corregir el valor de las constantes, se empleó la correlación de Bromley (1973) para la reacción global. El valor $B_{0}$ de la ecuación para cada reacción fue estimado con base en los valores reportados para la constante de equilibrio a diferentes fuerzas iónicas (Smith y Martell, 1976 y 1982).

C.4. - ESTIMACION DE LAS DIFUSIVIDADES LIBRES DE LAS ESPECIES

Uno de los problemas más comunes en el quehacer de la modelación de sistemas iónicos con transferencia de masa, es estimar las difusividades libres de las diferentes especies complejas. En este sistema donde se tiene diecisiete especies, la situación se torna difícil. Afortunadamente, como se están agrupando las especies por elemento o por grupo funcional, el error no es tan grande porque el resultado, ya puesto en las ecuaciones 
Apéndice $C$

de difusión, del cálculo individual es una forma de difusividad promedio ponderada de acuerdo con la concentración de cada especie en el balance. Además, debido a las constantes de equilibrio diferentes $Y$ a los excesos de algunos de los reactivos, se encuentra que la difusividad libre "promedio" en cada ecuación se acerca al valor de una sola especie. A pesar de esta situación se hizo un esfuerzo de estimar las difusividades de las diferentes especies.

Las difusividades de algunos compuestos se encuentran reportados en la literatura a $25^{\circ} \mathrm{C}$ :

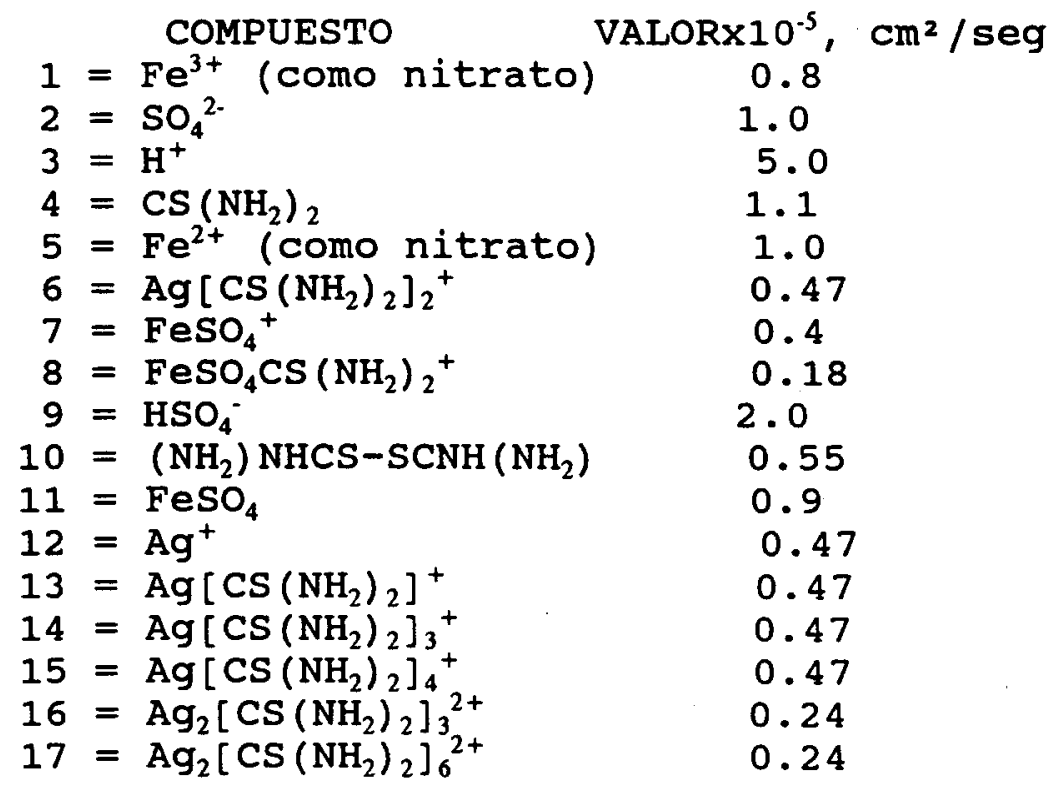

REFERENCIA

Rubcumintara y Han, 1990

Geankoplis, 1972

Geankoplis, 1972

Gubailovskii, 1972

Rubcumintara y Han, 1990 (Ver más adelante)

Rubcumintara y Han, 1990 (Ver más adelante)

Robinson $y$ stokes, 1959

(Ver más adelante)

Rubcumintara y Han, 1990

(Ver más adelante)

Shivrin y Shivrina,1976

(Ver más adelante)

"

En los casos de los compuestos no reportados en la literatura, se estimaron con diferentes criterios. Para el compuesto 8 $\left(\mathrm{FeSO}_{4} \mathrm{CS}\left(\mathrm{NH}_{2}\right)_{2}^{+}\right)$, sabiendo que es el complejo dominante de férrico, se calculó con los datos reportados por Pesic y Seal (1990) sobre un disco rotante usando la ecuación de Levich para tal propósito (Levich, 1973). Para los complejos de plata con tiourea, siendo dominante el tercer complejo $\left(\mathrm{AgTu}_{3}{ }^{+}\right)$, se igualaron los valores de 


\section{Apéndice $C$}

los demás complejos mononucleares al de éste. En el caso de los dimeros $\left(\left(\mathrm{NH}_{2}\right) \mathrm{NHCS}-\mathrm{SCNH}\left(\mathrm{NH}_{2}\right), A g_{2}\left[\mathrm{CS}\left(\mathrm{NH}_{2}\right)_{2}\right]_{3}{ }^{2+}\right.$ y $\left.\mathrm{Ag}{ }_{2}\left[\mathrm{CS}\left(\mathrm{NH}_{2}\right)_{2}\right]_{6}{ }^{2+}\right)$, el valor que se estimó para su difusividad libre era la mitad de la difusividad de sus respectivos monómeros.

\section{C.5. - ESTIMACION DE LA DIFUSIVIDAD EFECTIVA Y LA POROSIDAD}

Uno de las partes novedosas del programa, y que permite generalizar los cálculos para minerales de un contenido distinto de metales preciosos, es el estimador de la porosidad creada por la lixiviación, y consecuentemente, de la difusividad efectiva de las especies a través de la capa de inertes. Como se ha visto anteriormente, el programa utiliza la difusividad efectiva del ión ferrico libre para estimar el tiempo real de la lixiviación de un mineral en particular a partir del tiempo adimensional (aplicable a cualquier mineral). Cuando se cuentan con datos experimentales, se emplea un dato de conversión y tiempo para corregir el valor inicialmente estimado. La experiencia con el mineral empleado en este estudio es que el estimador predice una difusivadad efectiva "muy cercana" al valor corregido ( $\pm 60 \%)$.

El estimador empieza aproximando una porosidad creada por la lixiviación de plata. La porosidad creada depende de la ley de mineral y la estructura o fase metalúrgica. Este último factor dictará cual es el producto sólido que reemplaza a la plata una vez lixiviada; en el caso de argentita, este producto es el azufre y de plata metálica, no hay producto sólido.

El cálculo de la porosidad creada, $\epsilon_{\mathrm{C}}$, parte de un análisis de los porcientos de volumen ocupado por el metal de interés en el mineral total y por el producto. 
Apéndice $C$

$\epsilon_{\mathrm{c}}=\frac{\text { Volumen hueco }}{\text { Volumen total }}=\frac{(\text { Volumen de plata }- \text { Volumen de producto })}{\text { Volumen total de Mineral }}$

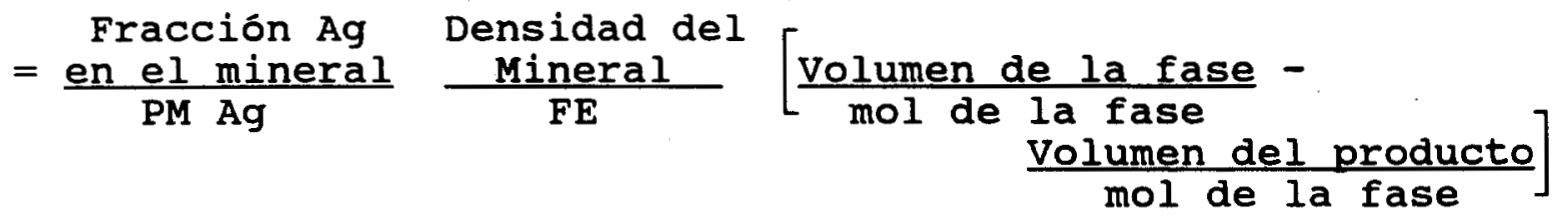

donde, $\mathrm{FE}=$ Factor estequiométrico que relaciona los moles de plata por mol de la fase metalúrgica, $\mathrm{Ag}_{2} \mathrm{~S}$ ó $\mathrm{Ag}^{\circ}$.

Una vez calculada la porosidad creada, se suma la porosidad original del mineral para calcular la porosidad total, $\epsilon$. Como la tortuosidad se aproxima por $1 / \epsilon$, entonces, la difusividad libre del ión férrico libre se multiplica por $\epsilon^{2}$ para encontrar su difusividad efectiva.

En cuanto a la aplicabilidad a otros minerales, el grado de éxito dependerá de la cantidad de otros metales que se lixivian junto con la plata. Por ejemplo, en el caso del mineral considerado en el presente estudio, el contenido de cobre es muy bajo y de éste se lixivió menos de $20 \%$, resultando en un error muy aceptable. Además, no se extrajo ninguno de los otros metales en cantidades apreciables por encontrarse en forma de sulfuros (galena, esfalerita $y$ pirita). En cambio, en otros minerales, especialmente aquellos con un alto porcentaje de óxidos, de éste cálculo pueden resultar difusividades efectivas sumamente bajas comparadas con la realidad. En este caso, una forma de corregir al cálculo, y que podría estimar más fielmente los tiempos reales de lixiviación, es considerar que los otros metales salen rápido y tomar en cuenta su contribución a la porosidad total en el valor dado a la porosidad original del mineral, además de llevar a cabo una corrección por la cantidad de agente oxidante consumida. 
Apéndice $C$

C.6. - EXTRACCION DE COBRE

La presencia en el mineral de otros metales, además de la plata, pudiera crear una competencia para la tiourea y el ión férrico contenidos en la solución. En el caso de sulfuros, el único metal que afectaría en este sentido al sistema es el cobre, debido al alto grado de reactividad y complejación de algunas de sus fases mineralógicas a las condiciones de la tioureación ácida.

La extracción de cobre para todos los experimentos se encuentra graficada como conversión fraccional versus tiempo en la siguiente figura. Se puede observar que el comportamiento es similar para todos los experimentos menos el que se llevó a cabo a $3^{\circ} \mathrm{C}$, experimento $19, y$ el experimento

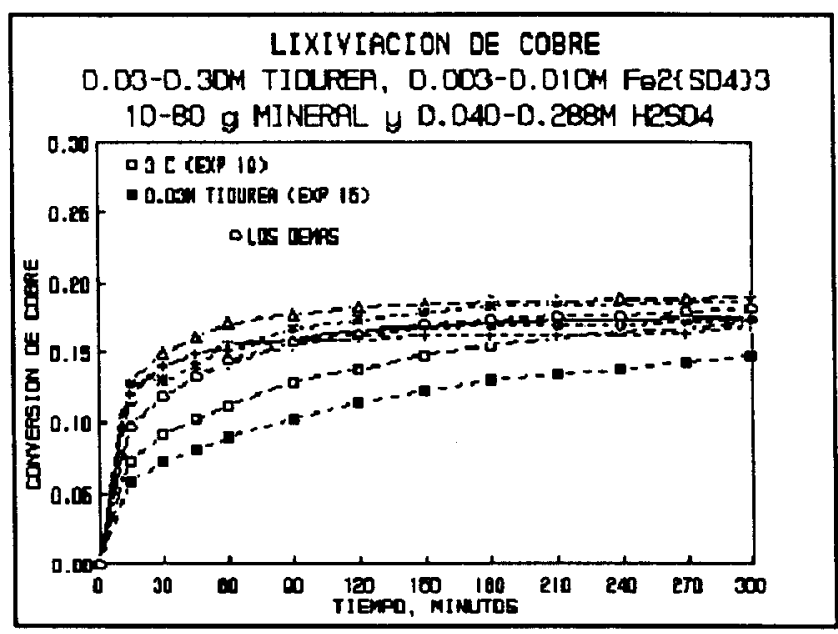
15 a $0.03 \mathrm{M}$ de tiourea. La conversión final llega a aproximadamente $18 \%$ en todos los casos, y el $90 \%$ de ésta en los primeros 90 minutos, independiente de las concentraciones de sulfato férrico, tiourea, ácido sulfúrico y el porciento de sólidos. Es únicamente más lenta en los casos de los experimentos 15 y 19. La causa probable de esta igualdad de comportamiento, es debido a la composición del mineral cuyo estructura dominante de cobre es la calcopirita la cual es prácticamente inerte a la lixiviación en estas condiciones (Rath et al., 1988). El poco cobre que se logra extraer posiblemente proviene de otras fases mineralógicas de sulfuros de cobre.

Esta rapidez para la extracción de cobre permite incorporar en el modelo, sin mucho error, el consumo de agente oxidante y tiourea 


\section{Apéndice $C$}

por este metal desde el inicio del programa, es decir que se considera instantánea su lixiviación, y consecuentemente, disminuye la concentración de reactivos disponibles para la oxidación de plata. Este hecho tiene implicaciones importantes cuando se aumenta el porciento de sólidos: se incrementa la cantidad de cobre en el sistema $y$ por ende, se reducen las concentraciones de tiourea y del oxidante. Con la pequeña cantidad de cobre lixiviable en el mineral considerado en el presente estudio, el efecto sobre la velocidad de lixiviación de plata tiene poca importancia, pero en minerales con un alto contenido de cobre pudiera influir mucho, especialmente a bajas concentraciones de tiourea. 
Apéndice D

\section{APENDICE D - DATOS EXPERIMENTALES Y SIMULACIONES}

Se llevaron a cabo todos los experimentos a $21^{\circ} \mathrm{C}$ con excepción a los experimentos 17 y 19. Los experimentos 1,3 y 16 fueron anulados debido a que no cerraron en el balance metalúrgico. En las figuras de cada experimento aparecen los datos experimentales como puntos, la simulación 1 (correspondiente al modelo original sin precipitación) y la simulación 2 (usando el modelo modificado que considera la precipitación de sólido) como lineas punteadas.

\begin{tabular}{|r|c|c|c|c|c|c|} 
EXP & $\begin{array}{c}\text { CONCENTRACIONES, } \\
\text { SULFATO } \\
\text { FERRICO }\end{array}$ & TIOUREA & $\begin{array}{c}\text { ACIDO } \\
\text { SULFURICO }\end{array}$ & $\begin{array}{c}\text { GRAMOS } \\
\text { MINERAL }\end{array}$ & T ${ }^{-}$C & PAGINAS \\
\hline 0 & 0.005 & 0.10 & 0.048 & 10 & 21 & $98-102$ \\
00 & 0.005 & 0.10 & 0.024 & 10 & 21 & $103-107$ \\
2 & 0.003 & 0.10 & 0.048 & 10 & 21 & $108-112$ \\
4 & 0.003 & 0.30 & 0.048 & 10 & 21 & $113-117$ \\
5 & 0.003 & 0.10 & 0.048 & 30 & 21 & $118-122$ \\
6 & 0.003 & 0.10 & 0.288 & 10 & 21 & $123-127$ \\
7 & 0.003 & 0.10 & 0.288 & 30 & 21 & $128-132$ \\
8 & 0.003 & 0.10 & 0.288 & 80 & 21 & $133-137$ \\
9 & 0.003 & 0.10 & 0.040 & 10 & 21 & $138-142$ \\
10 & 0.003 & 0.10 & 0.040 & 30 & 21 & $143-147$ \\
11 & 0.003 & 0.10 & 0.040 & 80 & 21 & $148-153$ \\
12 & 0.003 & 0.30 & 0.040 & 10 & 21 & $154-159$ \\
13 & 0.003 & 0.30 & 0.040 & 30 & 21 & $160-164$ \\
14 & 0.003 & 0.30 & 0.040 & 80 & 21 & $165-169$ \\
15 & 0.003 & 0.03 & 0.040 & 10 & 21 & $170-174$ \\
17 & 0.003 & 0.10 & 0.040 & 10 & 35 & $175-179$ \\
18 & 0.010 & 0.10 & 0.040 & 10 & 21 & $180-185$ \\
19 & 0.003 & 0.10 & 0.040 & 10 & 3 & $186-191$ \\
\hline
\end{tabular}




\section{Apéndice D - Experimento 0}

\section{EXPERIMENTO 0}

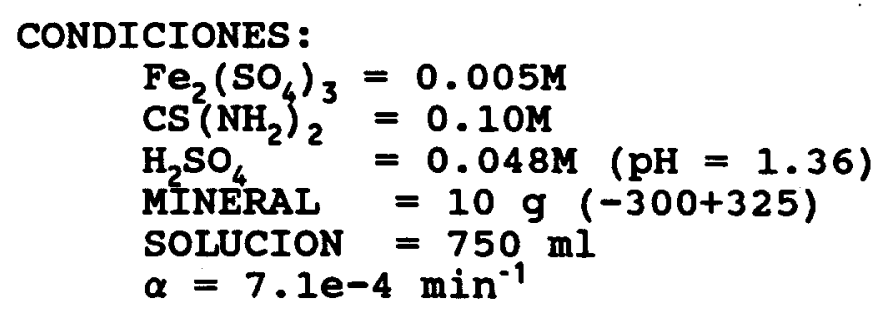

TIEMPO, MUESTRA, LECTURA, PPM CONVERSION minutos $\mathrm{ml}$ Ag $\mathrm{Cu}$ Ag $\mathrm{Cu}$

$\begin{array}{rrrrrr}12.0 & 6.0 & 16.1 & 4.0 & .164 & .100 \\ 28.0 & 6.0 & 23.6 & 5.1 & .239 & .127 \\ 43.0 & 6.0 & 29.0 & 5.9 & .293 & .146 \\ 60.0 & 6.0 & 33.9 & 6.3 & .342 & .156 \\ 88.0 & 12.0 & 39.4 & 7.0 & .396 & .173 \\ 120.0 & 6.0 & 46.0 & 7.3 & .460 & .180 \\ 148.0 & 6.0 & 49.0 & 7.4 & .488 & .182 \\ 185.0 & 6.0 & 55.1 & 7.6 & .546 & .187\end{array}$

RESIDUO DE Ag $=3.35 \mathrm{~kg} /$ ton

RESIDUO DE $\mathrm{Cu}=2.45 \mathrm{~kg} /$ ton

BALANCE METALURGICO DE Ag $=7.38 \mathrm{~kg} / \mathrm{ton}$

BALANCE METALURGICO DE $\mathrm{Cu}=3.01 \mathrm{~kg} /$ ton 


\title{
Apéndice D - Experimento 0
}

\author{
LIXIVIACION DE ARGENTITA \\ CARACTERISTICAS DEL MINERAL \\ GR POR TON DE AG $=.738 E+04$ \\ DENSIDAD DEL MINERAL $=5.20$ \\ RADIO DE LA PART, CM = .235E-02 \\ POROSIDAD $=0.000 \mathrm{E}+00$ \\ PROPORCION DE SOLIDO A LIQUIDO, KG/LT $=.133 \mathrm{E}-01$
}

CONSTANTES DE EQUILIBRIO A $21.00 \mathrm{C}$

CONCENTRACION INICIAL DE FE2(SO4) $3=.500 E-02$

CONCENTRACION INICIAL DE H2O2 $=0.000 \mathrm{E}+00$

CONCENTRACION INICIAL DE TIOUREA $=.100 \mathrm{E}+00$

GAMA $=\quad .281 \mathrm{E}-01$

VELOCIDAD DE DESCOMPOSICION DE TIOUREA = TIME* .710E-03

\footnotetext{
FE+3 FESO4+ FESO4 $(\mathrm{TH})+$ FEOH+2 FE(OH) 2+ FE2 (OH) 2+4 $\begin{array}{llllll}.245 \mathrm{E}-06 \quad .210 \mathrm{E}-08 & .100 \mathrm{E}-01 \quad .892 \mathrm{E}-08 \quad .482 \mathrm{E}-10 \quad .275 \mathrm{E}-13\end{array}$ $\begin{array}{llllll}.0000 & .0000 & 1.0000 & .0000 & .0000 & .0000\end{array}$

FUERZA IONICA DE LA SOLUTION $=.099$

CANTIDAD DE ION FERRICO UTILIZABLE = .100E-01

DIFUSIVIDAD EFECTIVA DE FE $+3=1.3380403174 \mathrm{E}-9$

ION FERRICO $=.194 \mathrm{E}-09$

SULFATO LIBRE $=.184 \mathrm{E}-01$

TIOUREA LIBRE $=.900 E-01$

TOTAL DE ACIDO ANADIDO $=.48 \mathrm{E}-01$

FADS $=.500 \mathrm{E}-02$

OXIDANTE CONSUMIDO POR COBRE $=.118 \mathrm{E}-03$

TIEMPO DE AJUSTE $=120$.

Ag2T6+2 $=.000258683674874$ LIMITE $=.00002190340224639$

PRECIPITACION DE Ag2SO4.3Tu.H2O

DIFUSUVIDAD EFECTIVA $=7.718143944871 \mathrm{E}-9$

PH REAL $=1.360837204844$

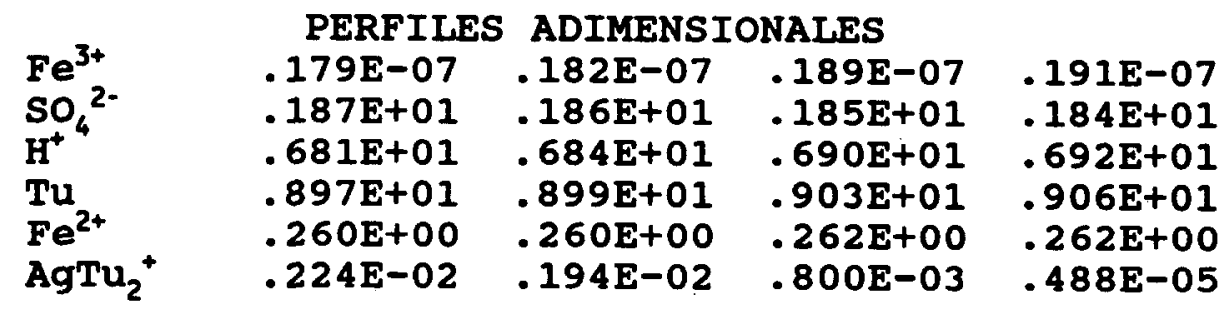


Apéndice D - Experimento 0

$\begin{array}{llcrrrrrr} & & \text { AG+ } & \text { AGT+ } & \text { AGT2+ } & \text { AGT3+ } & \text { AGT4+ } & \text { AG2T3+2 } & \text { AG2T6+2 } \\ \text { EN IA SUPER } & .4 \mathrm{E}-13 & .2 \mathrm{E}-06 & .2 \mathrm{E}-04 & .3 \mathrm{E}-03 & .3 \mathrm{E}-04 & .6 \mathrm{E}-03 & .16 \mathrm{E}-04 \\ \text { EN SOLUCION } & .8 \mathrm{E}-16 & .4 \mathrm{E}-09 & .5 \mathrm{E}-07 & .6 \mathrm{E}-06 & .7 \mathrm{E}-07 & .3 \mathrm{E}-08 & .78 \mathrm{E}-10\end{array}$

ADIM.TIEMPO RC/RP TIEMPO, MIN CONVERSION PH NUEVO

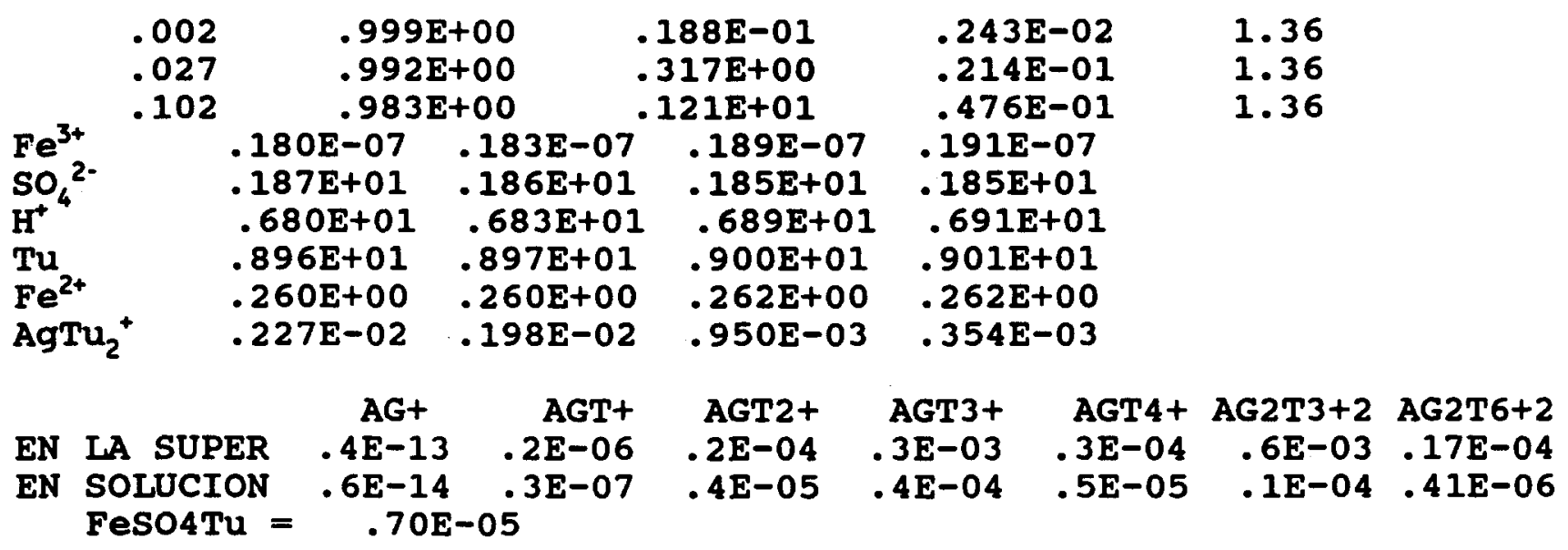

\begin{tabular}{|c|c|c|c|c|}
\hline $\begin{array}{c}\text { ADIM.TIEMPO } \\
.452 \\
.552 \\
.652 \\
.752 \\
.852 \\
1.002 \\
1.152 \\
1.302 \\
1.452 \\
1.602 \\
1.902 \\
2.202 \\
2.502 \\
2.802 \\
3.102 \\
3.602 \\
4.102 \\
4.602 \\
5.102\end{array}$ & $\begin{array}{c}\text { RC/RP } \\
.962 \mathrm{E}+00 \\
.958 \mathrm{E}+00 \\
.955 \mathrm{E}+00 \\
.952 \mathrm{E}+00 \\
.949 \mathrm{E}+00 \\
.944 \mathrm{E}+00 \\
.940 \mathrm{E}+00 \\
.937 \mathrm{E}+00 \\
.934 \mathrm{E}+00 \\
.930 \mathrm{E}+00 \\
.922 \mathrm{E}+00 \\
.917 \mathrm{E}+00 \\
.912 \mathrm{E}+00 \\
.907 \mathrm{E}+00 \\
.902 \mathrm{E}+00 \\
.893 \mathrm{E}+00 \\
.886 \mathrm{E}+00 \\
.880 \mathrm{E}+00 \\
.873 \mathrm{E}+00\end{array}$ & $\begin{array}{l}\text { TIEMPO, MIN } \\
.539 E+01 \\
.658 E+01 \\
.777 E+01 \\
.896 E+01 \\
.102 E+02 \\
.119 E+02 \\
.137 E+02 \\
.155 E+02 \\
.173 E+02 \\
.191 E+02 \\
.227 E+02 \\
.263 E+02 \\
.298 E+02 \\
.334 E+02 \\
.370 E+02 \\
.430 E+02 \\
.489 E+02 \\
.549 E+02 \\
.608 E+02\end{array}$ & $\begin{array}{c}\text { CONVERSION } \\
.102 \mathrm{E}+00 \\
.112 \mathrm{E}+00 \\
.122 \mathrm{E}+00 \\
.131 \mathrm{E}+00 \\
.139 \mathrm{E}+00 \\
.150 \mathrm{E}+00 \\
.160 \mathrm{E}+00 \\
.170 \mathrm{E}+00 \\
.179 \mathrm{E}+00 \\
.188 \mathrm{E}+00 \\
.204 \mathrm{E}+00 \\
.218 \mathrm{E}+00 \\
.231 \mathrm{E}+00 \\
.244 \mathrm{E}+00 \\
.256 \mathrm{E}+00 \\
.274 \mathrm{E}+00 \\
.291 \mathrm{E}+00 \\
.307 \mathrm{E}+00 \\
.322 \mathrm{E}+00\end{array}$ & $\begin{array}{l}\text { PH NUEVO } \\
1.36 \\
1.36 \\
1.36 \\
1.36 \\
1.36 \\
1.36 \\
1.36 \\
1.36 \\
1.36 \\
1.36 \\
1.36 \\
1.36 \\
1.36 \\
1.36 \\
1.36 \\
1.36 \\
1.36 \\
1.36 \\
1.36\end{array}$ \\
\hline
\end{tabular}




\section{Apéndice D - Experimento 0}

\begin{tabular}{|c|c|c|c|c|c|c|c|c|c|}
\hline $\begin{array}{l}\mathrm{Fe}^{3+} \\
\mathrm{SO}_{4}^{2-} \\
\mathrm{H}^{+} \\
\mathrm{Tu}^{2+} \\
\mathrm{Fe}^{2+} \\
\mathrm{AgTu}_{2}^{+}\end{array}$ & $\begin{array}{l}.188 \mathrm{E} \\
.187 \mathrm{E} \\
.679 \mathrm{E} \\
.8481 \\
.259 \mathrm{E} \\
.242 \mathrm{E}\end{array}$ & $\begin{array}{l}-07 \\
-01 \\
+01 \\
+01 \\
+00 \\
-02\end{array}$ & $\begin{array}{l}.1 \\
.1 \\
.6 \\
.1 \\
.2 \\
.2\end{array}$ & $\begin{array}{l}-07 \\
+01 \\
E+01 \\
E+01 \\
+00 \\
z-02\end{array}$ & $\begin{array}{l}.197 \mathrm{E}-07 \\
.185 \mathrm{E}+01 \\
.687 \mathrm{E}+01 \\
.851 \mathrm{E}+01 \\
.261 \mathrm{E}+00 \\
.127 \mathrm{E}-02\end{array}$ & $\begin{array}{l}.199 \mathrm{E}- \\
.185 \mathrm{E}+ \\
.689 \mathrm{Et} \\
.852 \mathrm{E} \\
.262 \mathrm{Et} \\
.871 \mathrm{E}-\end{array}$ & & & \\
\hline $\begin{array}{ll}\text { EN } & \text { LA } \\
\text { EN } & \text { SOL } \\
& \text { FeS }\end{array}$ & $\begin{array}{l}\text { SUPER } \\
\text { UCION } \\
\text { O4Tu = }\end{array}$ & $\begin{array}{r}\mathrm{A} \\
.5 \mathrm{E} \\
.2 \mathrm{E} \\
.\end{array}$ & $\begin{array}{l}+ \\
13 \\
13\end{array}$ & $\begin{array}{r}\text { AGT+ } \\
.2 \mathrm{E}-06 \\
7 \mathrm{E}-07\end{array}$ & $\begin{array}{r}\text { AGT2+ } \\
.2 E-04 \\
.9 E-05\end{array}$ & $\begin{array}{r}\text { AGT3+ } \\
.3 E-03 \\
.1 E-03\end{array}$ & $\begin{array}{r}\text { AGT4+ } \\
.3 \mathrm{E}-04 \\
.1 \mathrm{E}-04\end{array}$ & $\begin{array}{r}\text { AG2T3+2 } \\
.7 \mathrm{E}-03 \\
.9 \mathrm{E}-04\end{array}$ & $\begin{array}{r}\text { AG2T6+2 } \\
.17 \mathrm{E}-04 \\
.22 \mathrm{E}-05\end{array}$ \\
\hline
\end{tabular}

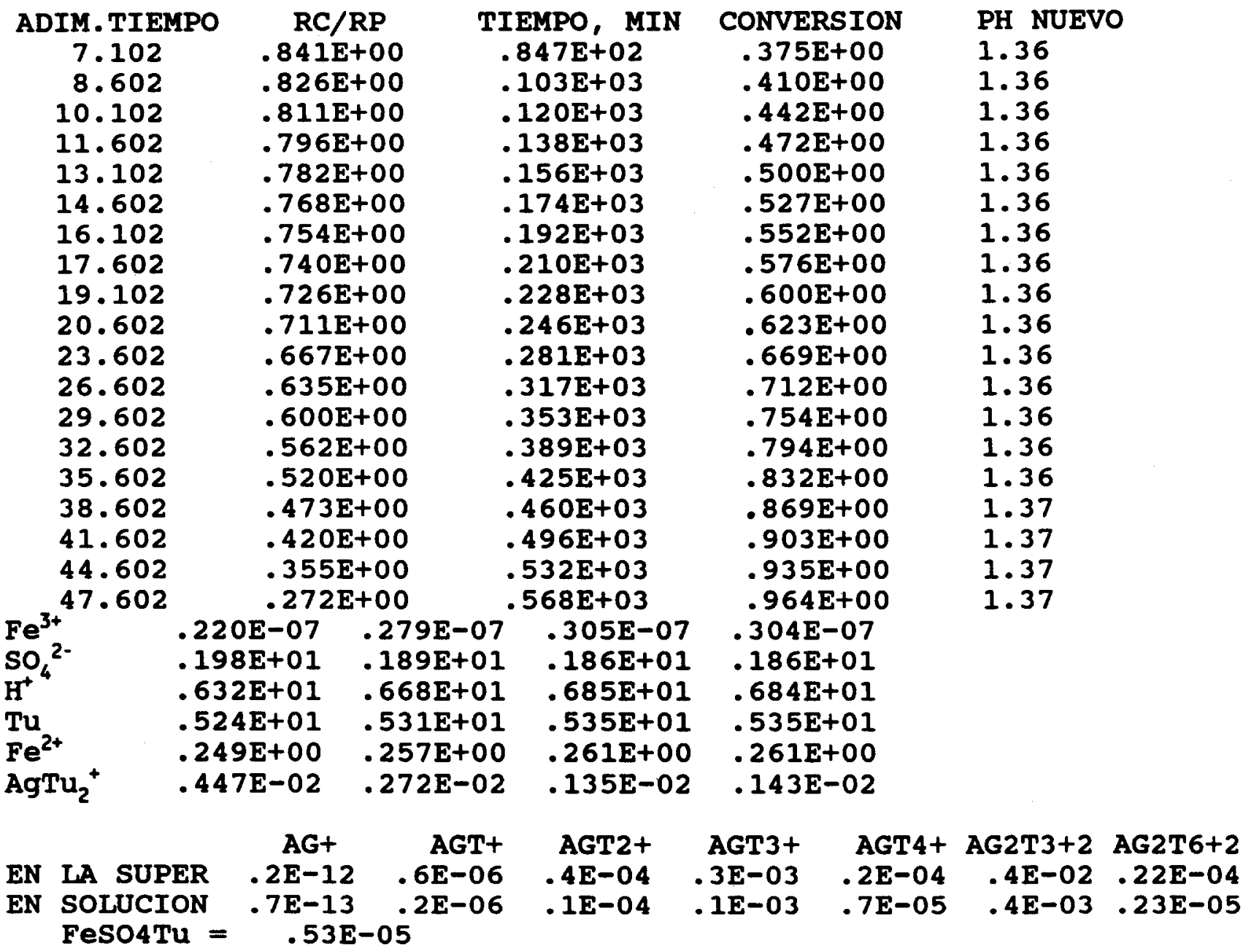

DIFUSIVIDAD EFECTIVA DE FE+3 $=7.718143944871 E-9$ CONV $=.15 \mathrm{E}-01 * t+-.12 \mathrm{E}-03 * t * * 2+.22 \mathrm{E}-06 * t * * 3$ 
Apéndice D - Experimento 0

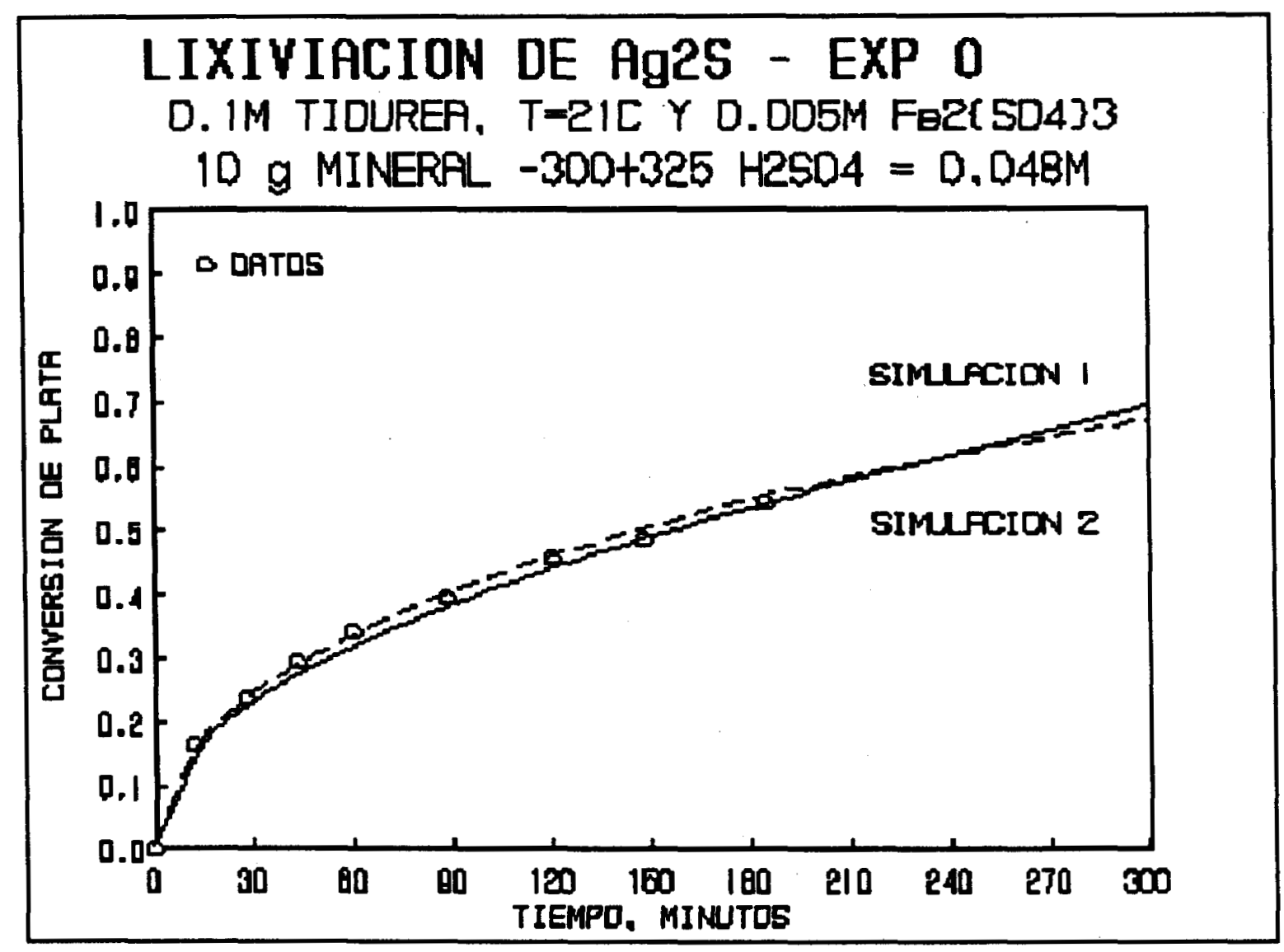


EXPERIMENTO 00

CONDICIONES :

$$
\begin{aligned}
& \mathrm{Fe}_{2}\left(\mathrm{SO}_{4}\right)_{3}=0.005 \mathrm{M} \\
& \mathrm{CS}^{2}\left(\mathrm{NH}_{2}\right)_{2}=0.10 \mathrm{M} \\
& \mathrm{H}_{2} \mathrm{SO}_{4}=0.024 \mathrm{M}(\mathrm{pH}=1.61) \\
& \mathrm{MINERAL}=10 \mathrm{~g}(-300+325) \\
& \text { SOLUCION }=750 \mathrm{mI} \\
& \alpha=7.84 \mathrm{e}-4 \mathrm{~min}^{-1}
\end{aligned}
$$

\begin{tabular}{crrrrr} 
TIEMPO, & MUESTRA, & \multicolumn{2}{c}{ LECTURA, } \\
minutos & ml & Ag & Cu & \multicolumn{2}{c}{ CONVERSION } \\
25.0 & 4.5 & 23.3 & 4.4 & .237 & .111 \\
40.0 & 4.5 & 30.1 & 5.1 & .305 & .128 \\
60.0 & 4.5 & 35.8 & 5.4 & .363 & .136 \\
75.0 & 4.5 & 39.9 & 5.8 & .404 & .145 \\
91.0 & 4.5 & 41.9 & 5.9 & .423 & .148 \\
120.0 & 16.5 & 47.3 & 5.3 & .477 & .133 \\
150.0 & 4.5 & 52.3 & 6.0 & .525 & .150 \\
180.0 & 4.5 & 55.0 & 6.6 & .551 & .164 \\
212.0 & 4.5 & 56.6 & 6.8 & .566 & .169 \\
240.0 & 16.5 & 58.1 & 6.9 & .580 & .171 \\
270.0 & 16.5 & 61.9 & 6.5 & .615 & .162
\end{tabular}

RESIDUO DE Ag $=2.84 \mathrm{~kg} /$ ton

RESIDUO DE Cu $=2.50 \mathrm{~kg} /$ ton

BALANCE METALURGICO DE Ag $=7.38 \mathrm{~kg} /$ ton

BALANCE METALURGICO DE $\mathrm{Cu}=2.98 \mathrm{~kg} / \mathrm{ton}$ 


\section{Apéndice D - Experimento 00}

\section{LIXIVIACION DE ARGENTITA}

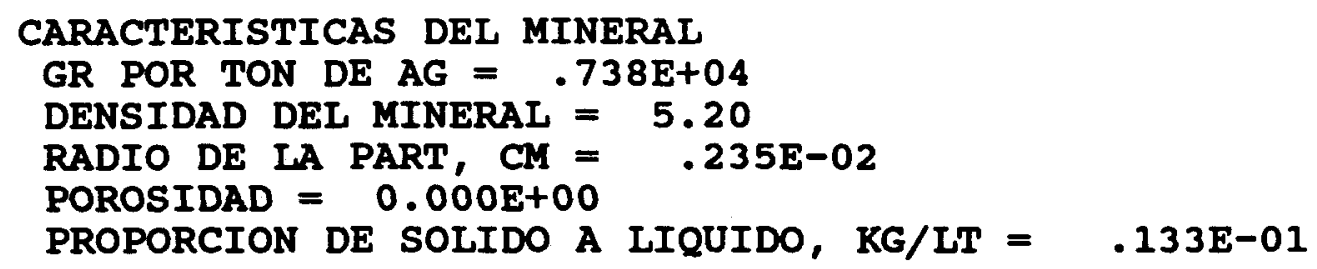

CONSTANTES DE EQUILIBRIO A $21.00 \mathrm{C}$

CONCENTRACION INICIAL DE FE2(SO4) $3=.500 E-02$

CONCENTRACION INICIAL DE H2O2 $=0.000 \mathrm{E}+00$

CONCENTRACION INICIAL DE TIOUREA $=.100 E+00$

GAMA $=.281 \mathrm{E}-01$

VELOCIDAD DE DESCOMPOSICION DE TIOUREA = TIME* .780E-03

$\begin{array}{rrrrrr}\text { FE+3 } & \text { FESO4+ } & \text { FESO4 }(\mathrm{TH})+ & \text { FEOH+2 } & \text { FE(OH) 2+ } & \text { FE2(OH) } 2+4 \\ .215 E-06 & .788 \mathrm{E}-09 & .100 \mathrm{O}-01 & .166 \mathrm{E}-07 & .162 \mathrm{E}-09 & .772 \mathrm{E}-13 \\ .0000 & .0000 & 1.0000 & .0000 & .0000 & .0000\end{array}$

FUERZA IONICA DE LA SOLUTION $=.065$

CANTIDAD DE ION FERRICO UTILIZABLE = .100E-01

DIFUSIVIDAD EFECTIVA DE FE+3 = $1.3380403174 \mathrm{E}-9$

ION FERRICO $=.137 \mathrm{E}-09$

SULFATO LIBRE $=.137 \mathrm{E}-01$

TIOUREA LIBRE $=.900 E-01$

TOTAL DE ACIDO ANADIDO = .24E-01

FADS $=.500 E-02$

OXIDANTE CONSUMIDO POR COBRE $=.101 \mathrm{E}-03$

\section{PERFILES ADIMENSIONALES}

TIEMPO DE AJUSTE $=180$.

Ag2T6+2 $=.003323103608627$ LIMITE $=.000003880864560717$

PRECIPITACION DE Ag2SO4.3Tu.H2O

DIFUSUVIDAD EFECTIVA $=6.785798600301 \mathrm{E}-10$

PH REAL $=1.610185245022$

$\begin{array}{lllll}\mathrm{Fe}^{3+} & .823 \mathrm{E}-09 & .411 \mathrm{E}-08 & .109 \mathrm{E}-07 & .136 \mathrm{E}-07 \\ \mathrm{SO}_{4}{ }^{2-} & .183 \mathrm{E}+01 & .171 \mathrm{E}+01 & .145 \mathrm{E}+01 & .137 \mathrm{E}+01 \\ \mathrm{H}^{+} & .259 \mathrm{E}+01 & .287 \mathrm{E}+01 & .369 \mathrm{E}+01 & .398 \mathrm{E}+01 \\ \mathrm{Tu} & .866 \mathrm{E}+01 & .872 \mathrm{E}+01 & .894 \mathrm{E}+01 & .905 \mathrm{E}+01 \\ \mathrm{Fe}^{2+} & .266 \mathrm{E}+00 & .278 \mathrm{E}+00 & .312 \mathrm{E}+00 & .323 \mathrm{E}+00 \\ \mathrm{AgTu}_{2}{ }^{+} & .924 \mathrm{E}-02 & .816 \mathrm{E}-02 & .398 \mathrm{E}-02 & .172 \mathrm{E}-04\end{array}$




\section{Apéndice D - Experimento 00}

$\begin{array}{llcrrrrrr} & \text { AGt } & \text { AGT+ } & \text { AGT2+ } & \text { AGT3+ } & \text { AGT4+ } & \text { AG2T3+2 } & \text { AG2T6+2 } \\ \text { EN LA SUPER } & .2 \mathrm{E}-12 & .7 \mathrm{E}-06 & .9 \mathrm{E}-04 & .1 \mathrm{E}-02 & .1 \mathrm{E}-03 & .1 \mathrm{E}-01 & .26 \mathrm{E}-03 \\ \text { EN SOLUCION } & .3 \mathrm{E}-15 & .1 \mathrm{E}-08 & .2 \mathrm{E}-06 & .2 \mathrm{E}-05 & .2 \mathrm{E}-06 & .3 \mathrm{E}-07 & .97 \mathrm{E}-09\end{array}$

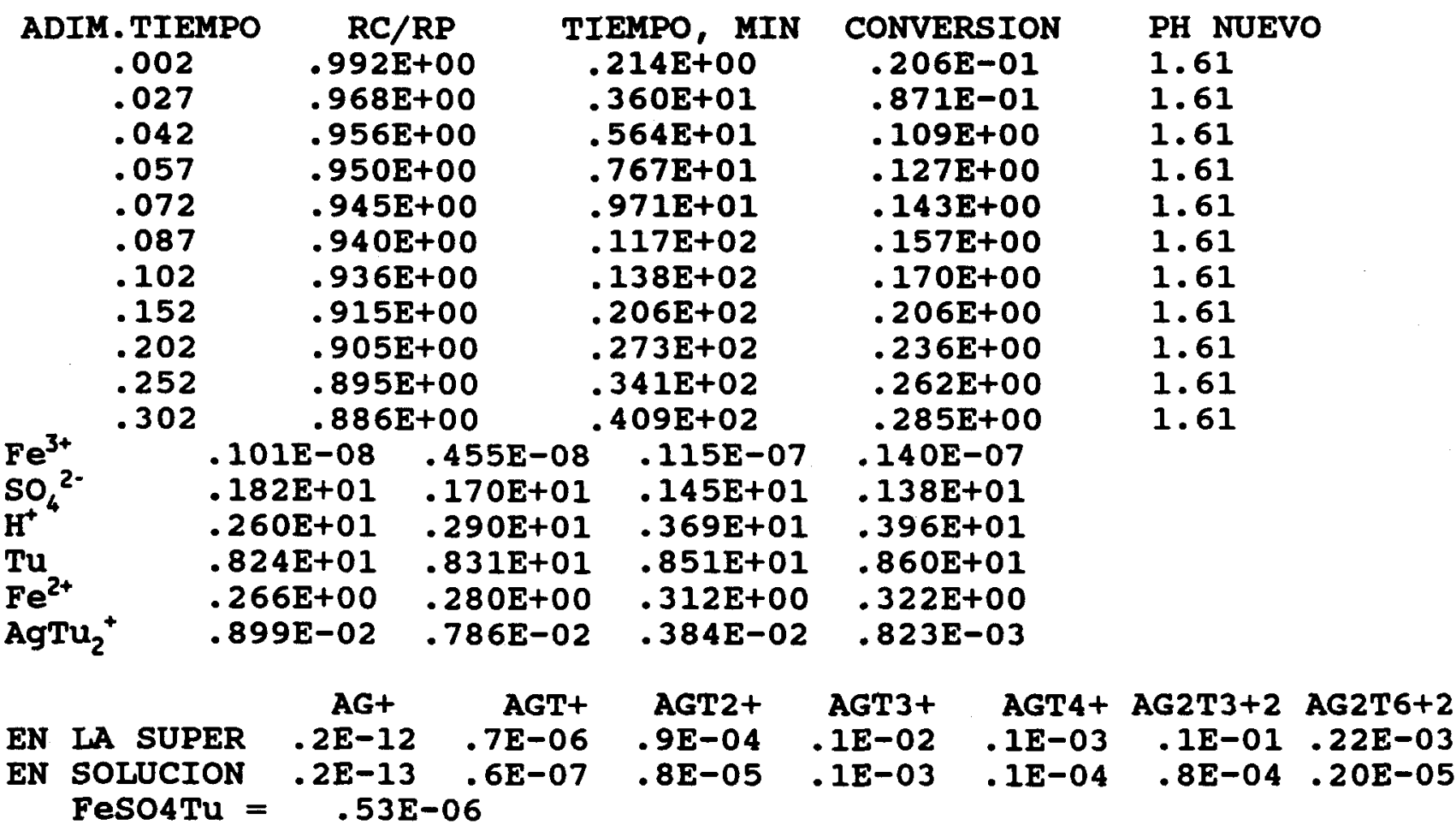

$\begin{array}{cc}\text { ADIM.TIEMPO } & \text { RC/RP } \\ .452 & .856 \mathrm{E}+00 \\ .552 & .843 \mathrm{E}+00 \\ .652 & .830 \mathrm{E}+00 \\ .752 & .818 \mathrm{E}+00 \\ .852 & .807 \mathrm{E}+00 \\ 1.002 & .786 \mathrm{E}+00 \\ 1.152 & .772 \mathrm{E}+00 \\ 1.302 & .757 \mathrm{E}+00 \\ 1.452 & .744 \mathrm{E}+00 \\ 1.602 & .731 \mathrm{E}+00 \\ 1.902 & .697 \mathrm{E}+00 \\ 2.202 & .675 \mathrm{E}+00 \\ 2.502 & .654 \mathrm{E}+00 \\ 2.802 & .635 \mathrm{E}+00 \\ 3.102 & .617 \mathrm{E}+00 \\ 3.602 & .596 \mathrm{E}+00\end{array}$

TIEMPO, MIN
$.613 E+02$
$.748 E+02$
$.884 E+02$
$.102 E+03$
$.116 E+03$
$.136 E+03$
$.156 E+03$
$.177 E+03$
$.197 E+03$
$.217 E+03$
$.258 E+03$
$.299 E+03$
$.339 E+03$
$.380 E+03$
$.421 E+03$
$.489 E+03$

CONVERSION

$.344 \mathrm{E}+00$

$.376 \mathrm{E}+00$

$.405 \mathrm{E}+00$

$.432 \mathrm{E}+00$

$.456 \mathrm{E}+00$

$.488 \mathrm{E}+00$

$.518 \mathrm{E}+00$

$.545 \mathrm{E}+00$

$.569 \mathrm{E}+00$

$.592 \mathrm{E}+00$

$.632 \mathrm{E}+00$

$.667 \mathrm{E}+00$

$.697 \mathrm{E}+00$

$.724 \mathrm{E}+00$

$.747 \mathrm{E}+00$

$.771 E+00$
PH NUEVO

1.61

1.61

1.61

1.61

1.61

1.61

1.61

1.61

1.61

1.62

1.62

1.62

1.62

1.62

1.62

1.62 


\section{Apéndice D - Experimento 00}

$\begin{array}{lllll}4.102 & .574 \mathrm{E}+00 & .556 \mathrm{E}+03 & .794 \mathrm{E}+00 & 1.62 \\ 4.602 & .554 \mathrm{E}+00 & .624 \mathrm{E}+03 & .814 \mathrm{E}+00 & 1.62 \\ 5.102 & .534 \mathrm{E}+00 & .692 \mathrm{E}+03 & .833 \mathrm{E}+00 & 1.62\end{array}$

$\begin{array}{lllll}\mathrm{Fe}^{+3} & .133 \mathrm{E}-07 & .179 \mathrm{E}-07 & .255 \mathrm{E}-07 & .272 \mathrm{E}-07 \\ \mathrm{SO}_{4}{ }^{2-} & .162 \mathrm{E}+01 & .153 \mathrm{E}+01 & .141 \mathrm{E}+01 & .139 \mathrm{E}+01 \\ \mathrm{H}^{+} & .313 \mathrm{E}+01 & .339 \mathrm{E}+01 & .382 \mathrm{E}+01 & .392 \mathrm{E}+01 \\ \mathrm{Tu}^{2+} & .408 \mathrm{E}+01 & .413 \mathrm{E}+01 & .422 \mathrm{E}+01 & .424 \mathrm{E}+01 \\ \mathrm{Fe}^{2+} & .290 \mathrm{E}+00 & .301 \mathrm{E}+00 & .317 \mathrm{E}+00 & .321 \mathrm{E}+00 \\ \mathrm{AgTu}_{2}{ }^{+} & .497 \mathrm{E}-02 & .409 \mathrm{E}-02 & .204 \mathrm{E}-02 & .121 \mathrm{E}-02\end{array}$

$\begin{array}{lccrrrrrr} & & \text { AGt } & \text { AGT+ } & \text { AGT2+ } & \text { AGT3+ } & \text { AGT4+ } & \text { AG2T3+2 } & \text { AG2T6+2 } \\ \text { EN LA SUPER } & .4 \mathrm{E}-12 & .8 \mathrm{E}-06 & .5 \mathrm{E}-04 & .3 \mathrm{E}-03 & .1 \mathrm{E}-04 & .6 \mathrm{E}-02 & .16 \mathrm{E}-04 \\ \text { EN SOLUCION } & .9 \mathrm{E}-13 & .2 \mathrm{E}-06 & .1 \mathrm{E}-04 & .7 \mathrm{E}-04 & .4 \mathrm{E}-05 & .3 \mathrm{E}-03 & .10 \mathrm{E}-05\end{array}$

ADIM.TIEMPO RC/RP TIEMPO, MIN CONVERSION PH NUEVO

$\begin{array}{rrrrr}7.102 & .429 \mathrm{E}+00 & .963 \mathrm{E}+03 & .894 \mathrm{E}+00 & 1.62 \\ 8.602 & .386 \mathrm{E}+00 & .117 \mathrm{E}+04 & .923 \mathrm{E}+00 & 1.62 \\ 10.102 & .334 \mathrm{E}+00 & .137 \mathrm{E}+04 & .948 \mathrm{E}+00 & 1.62 \\ 11.602 & .266 \mathrm{E}+00 & .157 \mathrm{E}+04 & .969 \mathrm{E}+00 & 1.62 \\ 14.602 & .107 \mathrm{E}+00 & .198 \mathrm{E}+04 & .995 \mathrm{E}+00 & 1.62\end{array}$

DIFUSIVIDAD EFECTIVA DE FE+3 $=6.785798600301 \mathrm{E}-10$ CONV $=.16 \mathrm{E}-01 * t+-.14 \mathrm{E}-03 * t * 2+2+.20 \mathrm{E}-06 * t * * 3$ 


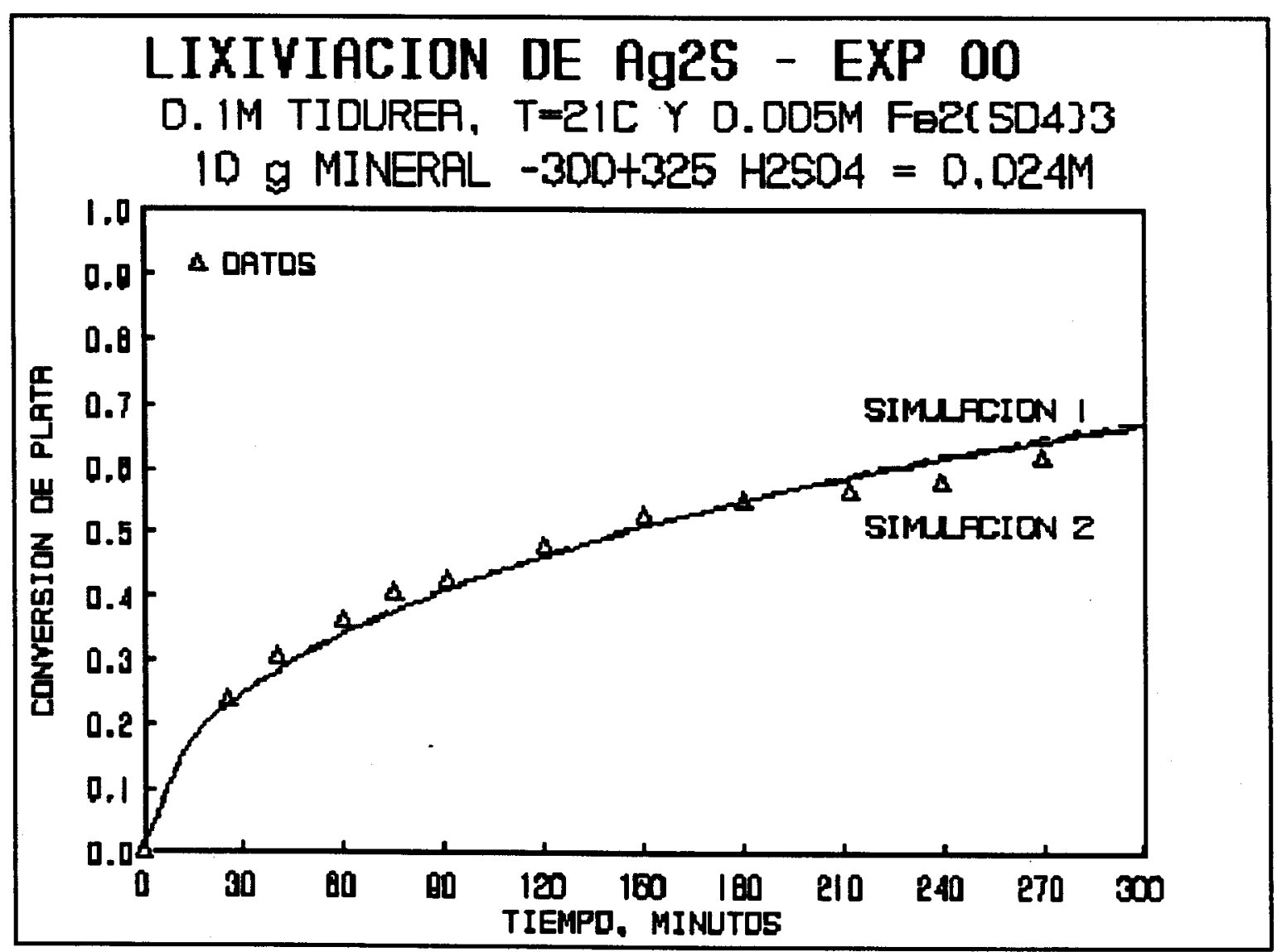




\section{Apéndice D - Experimento 2}

EXPERIMENTO 2

CONDICIONES :

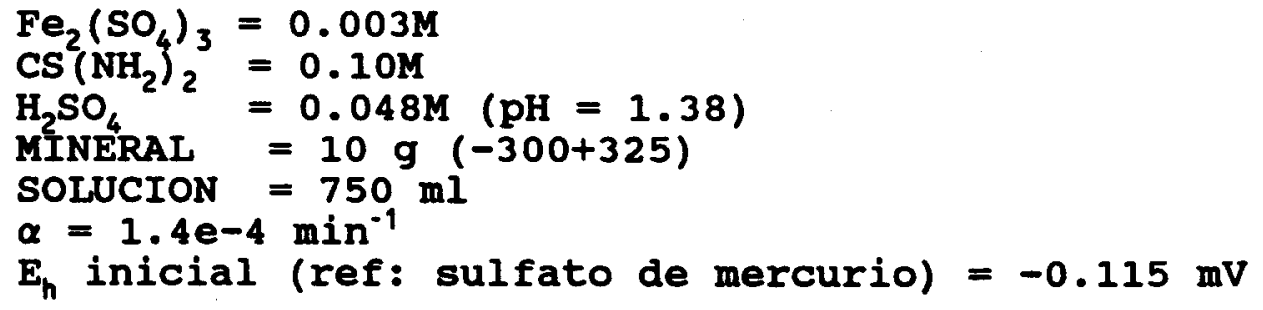

\begin{tabular}{|c|c|c|c|c|c|c|}
\hline $\begin{array}{l}\text { TIEMPO, } \\
\text { minutos }\end{array}$ & $\begin{array}{c}\text { MUESTRA, } \\
\mathrm{ml}\end{array}$ & $\begin{array}{l}\text { LECTURA } \\
\text { Ag }\end{array}$ & Pu & $\begin{array}{c}\text { CONV } \\
\text { Ag }\end{array}$ & $\begin{array}{l}\text { ION } \\
\mathrm{Cu}\end{array}$ & $\begin{array}{l}E_{h{ }^{\prime}} \\
m \mathbf{m V}^{-}\end{array}$ \\
\hline $\begin{array}{r}1.0 \\
15.0 \\
30.0 \\
45.0 \\
60.0 \\
90.0 \\
120.0 \\
143.0 \\
150.0 \\
180.0 \\
210.0 \\
240.0 \\
270.0 \\
290.0 \\
300.0\end{array}$ & $\begin{array}{r}18 . \\
9 . \\
6 . \\
6 . \\
6 . \\
9 . \\
6 . \\
12 . \\
6 . \\
6 . \\
6 . \\
6 . \\
6 . \\
12 . \\
6 .\end{array}$ & $\begin{array}{l}6.5 \\
22.5 \\
31.5 \\
37.8 \\
44.1 \\
52.8 \\
59.4 \\
61.0 \\
61.6 \\
67.1 \\
69.3 \\
70.4 \\
73.4 \\
74.0 \\
74.8\end{array}$ & $\begin{array}{l}2.1 \\
5.1 \\
5.2 \\
5.7 \\
6.2 \\
6.7 \\
7.0 \\
7.2 \\
7.2 \\
7.4 \\
7.4 \\
7.8 \\
7.9 \\
7.7 \\
7.6\end{array}$ & $\begin{array}{l}.0663 \\
.2257 \\
.3143 \\
.3758 \\
.4368 \\
.5202 \\
.5827 \\
.5978 \\
.6033 \\
.6536 \\
.6736 \\
.6834 \\
.7101 \\
.7154 \\
.7224\end{array}$ & $\begin{array}{l}.053 \\
.128 \\
.130 \\
.142 \\
.154 \\
.166 \\
.173 \\
.178 \\
.178 \\
.183 \\
.183 \\
.192 \\
.194 \\
.189 \\
.187\end{array}$ & $\begin{array}{l}-.160 \\
-.223 \\
-.224 \\
-.225 \\
-.227 \\
-.229 \\
-.231 \\
-.232 \\
-.232 \\
-.234 \\
-.235 \\
-.236 \\
-.236 \\
-.237 \\
-.237\end{array}$ \\
\hline
\end{tabular}

RESIDUO DE Ag $=2.04 \mathrm{~kg} /$ ton

RESIDUO DE $\mathrm{Cu}=2.40 \mathrm{~kg} /$ ton

BALANCE METALURGICO DE Ag $=7.35 \mathrm{~kg} / \mathrm{ton}$

BALANCE METALURGICO DE $\mathrm{Cu}=2.95 \mathrm{~kg} / \mathrm{ton}$ 


\section{Apéndice D - Experimento 2}

LIXIVIACION DE ARGENTITA

CARACTERISTICAS DEL MINERAL GR POR TON DE AG $=.735 E+04$ DENSIDAD DEL MINERAL $=\mathbf{5 . 2 0}$ RADIO DE LA PART, $\mathrm{CM}=.235 \mathrm{E}-02$ POROSIDAD $=0.000 \mathrm{E}+00$ PROPORCION DE SOLIDO A LIQUIDO, KG/LT $=.133 E-01$

CONSTANTES DE EQUILIBRIO A $21.00 \mathrm{C}$

CONCENTRACION INICIAL DE FE2 $\left(\mathrm{SO}_{4}\right) 3=.310 \mathrm{E}-02$

CONCENTRACION INICIAL DE H2O2 $=0.000 E+00$ CONCENTRACION INICIAL DE TIOUREA $=.100 \mathrm{E}+00$ GAMA $=\quad .175 \mathrm{E}-01$

VELOCIDAD DE DESCOMPOSICION DE TIOUREA = TIME* .140E-03

$\begin{array}{crrrrr}\text { FE+3 } & \text { FESO4+ } & \text { FESO4 (TH) } & \text { FEOH+2 } & \text { FE(OH) 2+ } & \text { FE2(OH) } 2+4 \\ .145 \mathrm{E}-06 & .100 \mathrm{O}-08 & .620 \mathrm{O}-02 & .578 \mathrm{E}-08 & .329 \mathrm{E}-10 & .110 \mathrm{O}-13 \\ .0000 & .0000 & 1.0002 & .0000 & .0000 & .0000\end{array}$

FUERZA IONICA DE LA SOLUTION $=.090$

CANTIDAD DE ION FERRICO UTILIZABLE $=.620 \mathrm{E}-02$

DIFUSIVIDAD EFECTIVA DE FE+3 $=1.327184051357 E-9$

ION FERRICO $=.907 \mathrm{E}-10$

SULFATO LIBRE $=.173 \mathrm{E}-01$

TIOUREA LIBRE $=\quad .938 \mathrm{E}-01$

TOTAL DE ACIDO ANADIDO $=.47 \mathrm{E}-01$

FADS $=.310 \mathrm{E}-02$

OXIDANTE CONSUMIDO POR COBRE $=.115 \mathrm{E}-03$

\section{PERFILES ADIMENSIONALES}

TIEMPO DE AJUSTE $=240$.

Ag2T6+2 $=.0001790351830739$ LIMITE $=.00004498365336968$

PRECIPITACION DE Ag2SO4.3Tu.H2O

DIFUSUVIDAD EFECTIVA $=1.529672625108 \mathrm{E}-9$

\begin{tabular}{lcccc}
\multicolumn{5}{c}{ PH REAL $=1.382742224771$} \\
$\mathrm{Fe}^{+3}$ & $.242 \mathrm{E}-08$ & $.610 \mathrm{E}-08$ & $.124 \mathrm{E}-07$ & $.144 \mathrm{E}-07$ \\
$\mathrm{SO}_{4}{ }^{2-}$ & $.314 \mathrm{E}+01$ & $.306 \mathrm{E}+01$ & $.286 \mathrm{E}+01$ & $.279 \mathrm{E}+01$ \\
$\mathrm{H}^{+}$ & $.918 \mathrm{E}+01$ & $.950 \mathrm{E}+01$ & $.104 \mathrm{E}+02$ & $.107 \mathrm{E}+02$ \\
$\mathrm{Tu}^{2+}$ & $.148 \mathrm{E}+02$ & $.149 \mathrm{E}+02$ & $.151 \mathrm{E}+02$ & $.152 \mathrm{E}+02$ \\
$\mathrm{Fe}^{2+}$ & $.253 \mathrm{E}+00$ & $.258 \mathrm{E}+00$ & $.270 \mathrm{E}+00$ & $.275 \mathrm{E}+00$ \\
$\mathrm{AgTu}_{2}{ }^{+}$ & $.115 \mathrm{E}-01$ & $.101 \mathrm{E}-01$ & $.480 \mathrm{E}-02$ & $.206 \mathrm{E}-04$
\end{tabular}


Apéndice D - Experimento 2

$\begin{array}{llcrrrrrr} & & \text { AG+ } & \text { AGT+ } & \text { AGT2+ } & \text { AGT3+ } & \text { AGT4+ } & \text { AG2T3+2 } & \text { AG2T6+2 } \\ \text { EN LA SUPER } & .1 \mathrm{E}-12 & .5 \mathrm{E}-06 & .7 \mathrm{E}-04 & .9 \mathrm{E}-03 & .1 \mathrm{E}-03 & .6 \mathrm{E}-02 & .17 \mathrm{E}-03 \\ \text { EN SOLUCION } & .2 \mathrm{E}-15 & .9 \mathrm{E}-09 & .1 \mathrm{E}-06 & .2 \mathrm{E}-05 & .2 \mathrm{E}-06 & .2 \mathrm{E}-07 & .58 \mathrm{E}-09 \\ & \text { FeSO4TU }= & .68 \mathrm{E}-06 & & & & & & \end{array}$

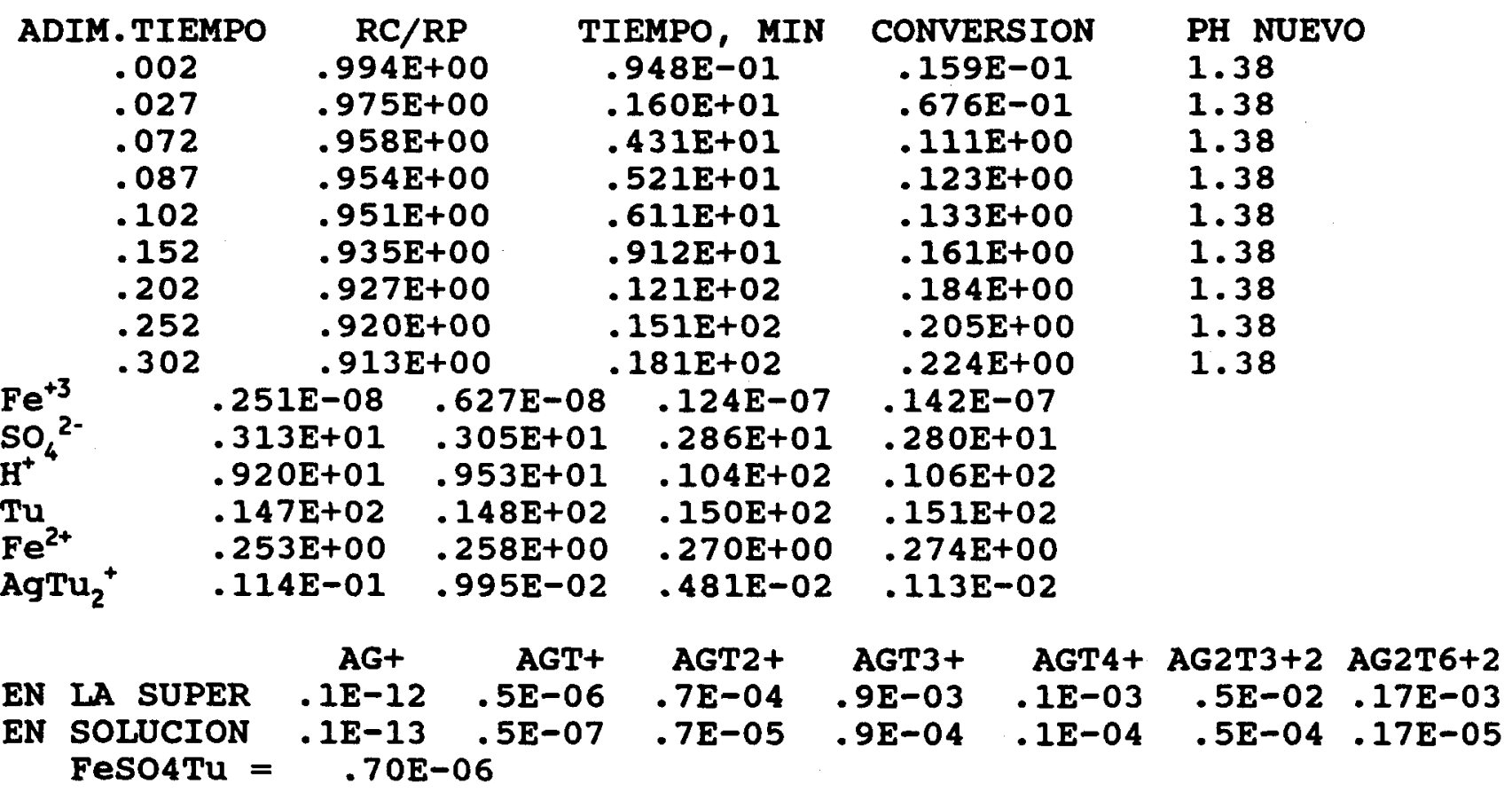

$\begin{array}{ccccl}\text { ADIM.TIEMPO } & \text { RC/RP } & \text { TIEMPO, MIN } & \text { CONVERSION } & \text { PH NUEVO } \\ .452 & .891 \mathrm{E}+00 & .272 \mathrm{E}+02 & .270 \mathrm{E}+00 & 1.38 \\ .552 & .881 \mathrm{E}+00 & .332 \mathrm{E}+02 & .297 \mathrm{E}+00 & 1.38 \\ .652 & .871 \mathrm{E}+00 & .392 \mathrm{E}+02 & .320 \mathrm{E}+00 & 1.38 \\ .752 & .862 \mathrm{E}+00 & .452 \mathrm{E}+02 & .342 \mathrm{E}+00 & 1.38 \\ .852 & .854 \mathrm{E}+00 & .512 \mathrm{E}+02 & .362 \mathrm{E}+00 & 1.38 \\ 1.002 & .839 \mathrm{E}+00 & .603 \mathrm{E}+02 & .389 \mathrm{E}+00 & 1.38 \\ 1.152 & .828 \mathrm{E}+00 & .693 \mathrm{E}+02 & .414 \mathrm{E}+00 & 1.38 \\ 1.302 & .817 \mathrm{E}+00 & .783 \mathrm{E}+02 & .436 \mathrm{E}+00 & 1.38 \\ 1.452 & .807 \mathrm{E}+00 & .873 \mathrm{E}+02 & .457 \mathrm{E}+00 & 1.39 \\ 1.602 & .798 \mathrm{E}+00 & .964 \mathrm{E}+02 & .477 \mathrm{E}+00 & 1.39 \\ 1.902 & .773 \mathrm{E}+00 & .114 \mathrm{E}+03 & .512 \mathrm{E}+00 & 1.39 \\ 2.202 & .756 \mathrm{E}+00 & .132 \mathrm{E}+03 & .543 \mathrm{E}+00 & 1.39 \\ 2.502 & .740 \mathrm{E}+00 & .151 \mathrm{E}+03 & .572 \mathrm{E}+00 & 1.39 \\ 2.802 & .725 \mathrm{E}+00 & .169 \mathrm{E}+03 & .598 \mathrm{E}+00 & 1.39 \\ 3.102 & .711 \mathrm{E}+00 & .187 \mathrm{E}+03 & .622 \mathrm{E}+00 & 1.39 \\ 3.602 & .680 \mathrm{E}+00 & .217 \mathrm{E}+03 & .658 \mathrm{E}+00 & 1.39 \\ 4.102 & .659 \mathrm{E}+00 & .247 \mathrm{E}+03 & .690 \mathrm{E}+00 & 1.39 \\ 4.602 & .638 \mathrm{E}+00 & .277 \mathrm{E}+03 & .719 \mathrm{E}+00 & 1.39 \\ 5.102 & .617 \mathrm{E}+00 & .307 \mathrm{E}+03 & .745 \mathrm{E}+00 & 1.39\end{array}$




\section{Apéndice D - Experimento 2}

\begin{tabular}{|c|c|c|c|c|c|c|c|c|}
\hline \multirow{2}{*}{$\begin{array}{l}\mathrm{Fe}^{+3} \\
\mathrm{SO}_{4}{ }^{2-} \\
\mathrm{H}^{+} \\
\mathrm{Tu}^{2+} \\
\mathrm{Fe}^{2+} \\
\mathrm{AgTu}_{2}^{+} \\
\text {EN LA } \\
\text { EN SOL }\end{array}$} & \multicolumn{2}{|c|}{$\begin{array}{l}.330 \mathrm{E}-08 \\
.311 \mathrm{E}+01 \\
.928 \mathrm{E}+01 \\
.140 \mathrm{E}+02 \\
.254 \mathrm{E}+00 \\
.108 \mathrm{E}-01\end{array}$} & $\begin{array}{l}.759 \mathrm{E}-08 \\
.301 \mathrm{E}+01 \\
.968 \mathrm{E}+01 \\
.141 \mathrm{E}+02 \\
.260 \mathrm{E}+00 \\
.901 \mathrm{E}-02\end{array}$ & $\begin{array}{l}.130 \mathrm{E}-07 \\
.285 \mathrm{E}+01 \\
.104 \mathrm{E}+02 \\
.142 \mathrm{E}+02 \\
.271 \mathrm{E}+00 \\
.439 \mathrm{E}-02\end{array}$ & \multicolumn{2}{|c|}{$\begin{array}{l}.142 \mathrm{E}-07 \\
.281 \mathrm{E}+01 \\
.106 \mathrm{E}+02 \\
.143 \mathrm{E}+02 \\
.273 \mathrm{E}+00 \\
.233 \mathrm{E}-02\end{array}$} & \multirow[b]{2}{*}{$\begin{array}{r}\text { AG2T } 3+2 \\
.5 \mathrm{E}-02 \\
.2 \mathrm{E}-03\end{array}$} & \multirow[b]{2}{*}{$\begin{array}{l}\text { AG2T6+2 } \\
.13 \mathrm{E}-03 \\
.66 \mathrm{E}-05\end{array}$} \\
\hline & & $\begin{array}{r}\text { AG+ } \\
.1 \mathrm{E}-12 \\
.2 \mathrm{E}-13 \\
.86 \mathrm{E}\end{array}$ & $\begin{array}{l}\text { AGT+ } \\
.5 E-06 \\
.1 E-06 \\
06\end{array}$ & $\begin{array}{r}\text { AGT2+ } \\
.7 E-04 \\
.1 E-04\end{array}$ & $\begin{array}{r}\text { AGT3+ } \\
.8 \mathrm{E}-03 \\
.2 \mathrm{E}-03\end{array}$ & $\begin{array}{r}\text { AGT4+ } \\
.8 E-04 \\
.2 E-04\end{array}$ & & \\
\hline
\end{tabular}

$\begin{array}{ccccc}\text { ADIM. TIEMPO } & \text { RC/RP } & \text { TIEMPO, MIN } & \text { CONVERSION } & \text { PH NUE } \\ 7.102 & .511 E+00 & .427 \mathrm{E}+03 & .828 \mathrm{E}+00 & 1.39 \\ 8.602 & .457 \mathrm{E}+00 & .518 \mathrm{E}+03 & .874 \mathrm{E}+00 & 1.39 \\ 10.102 & .402 \mathrm{E}+00 & .608 \mathrm{E}+03 & .911 \mathrm{E}+00 & 1.39 \\ 11.602 & .345 \mathrm{E}+00 & .698 \mathrm{E}+03 & .941 \mathrm{E}+00 & 1.39 \\ 13.102 & .284 \mathrm{E}+00 & .788 \mathrm{E}+03 & .964 \mathrm{E}+00 & 1.39 \\ 14.602 & .213 \mathrm{E}+00 & .879 \mathrm{E}+03 & .981 \mathrm{E}+00 & 1.39 \\ 16.102 & .116 \mathrm{E}+00 & .969 \mathrm{E}+03 & .994 \mathrm{E}+00 & 1.39\end{array}$

$\begin{array}{lllll}\mathrm{Fe}^{+3} & .559 \mathrm{E}-08 & .159 \mathrm{E}-07 & .161 \mathrm{E}-07 & .151 \mathrm{E}-07 \\ \mathrm{SO}_{4}{ }^{2-} & .307 \mathrm{E}+01 & .282 \mathrm{E}+01 & .279 \mathrm{E}+01 & .282 \mathrm{E}+01 \\ \mathrm{H}^{+} & .941 \mathrm{E}+01 & .105 \mathrm{E}+02 & .106 \mathrm{E}+02 & .105 \mathrm{E}+02 \\ \mathrm{Tu} & .126 \mathrm{E}+02 & .128 \mathrm{E}+02 & .129 \mathrm{E}+02 & .129 \mathrm{E}+02 \\ \mathrm{Fe}^{2+} & .256 \mathrm{E}+00 & .273 \mathrm{E}+00 & .275 \mathrm{E}+00 & .273 \mathrm{E}+00 \\ \mathrm{AgTu}_{2}{ }^{+} & .981 \mathrm{E}-02 & .323 \mathrm{E}-02 & .101 \mathrm{E}-02 & .264 \mathrm{E}-02\end{array}$

$\begin{array}{llcrrrrrr} & & \text { AG+ } & \text { AGT+ } & \text { AGT2+ } & \text { AGT3+ } & \text { AGT4+ } & \text { AG2T3+2 } & \text { AG2T6+2 } \\ \text { EN IA SUPER } & .1 \mathrm{E}-12 & .5 \mathrm{E}-06 & .6 \mathrm{E}-04 & .7 \mathrm{E}-03 & .6 \mathrm{E}-04 & .5 \mathrm{E}-02 & .89 \mathrm{E}-04 \\ \text { EN SOLUCION } & .3 \mathrm{E}-13 & .1 \mathrm{E}-06 & .2 \mathrm{E}-04 & .2 \mathrm{E}-03 & .2 \mathrm{E}-04 & .3 \mathrm{E}-03 & .68 \mathrm{E}-05 \\ & \text { FeSO4Tu }= & .13 \mathrm{E}-05 & & & & & \end{array}$

DIFUSIVIDAD EFECTIVA DE FE+3 $=1.529672625108 \mathrm{E}-9$

$\mathrm{CONV}=.23 \mathrm{E}-01 * t+-.28 \mathrm{E}-03 * t * * 2+.64 \mathrm{E}-06 * t * * 3$ 
Apéndice D - Experimento 2

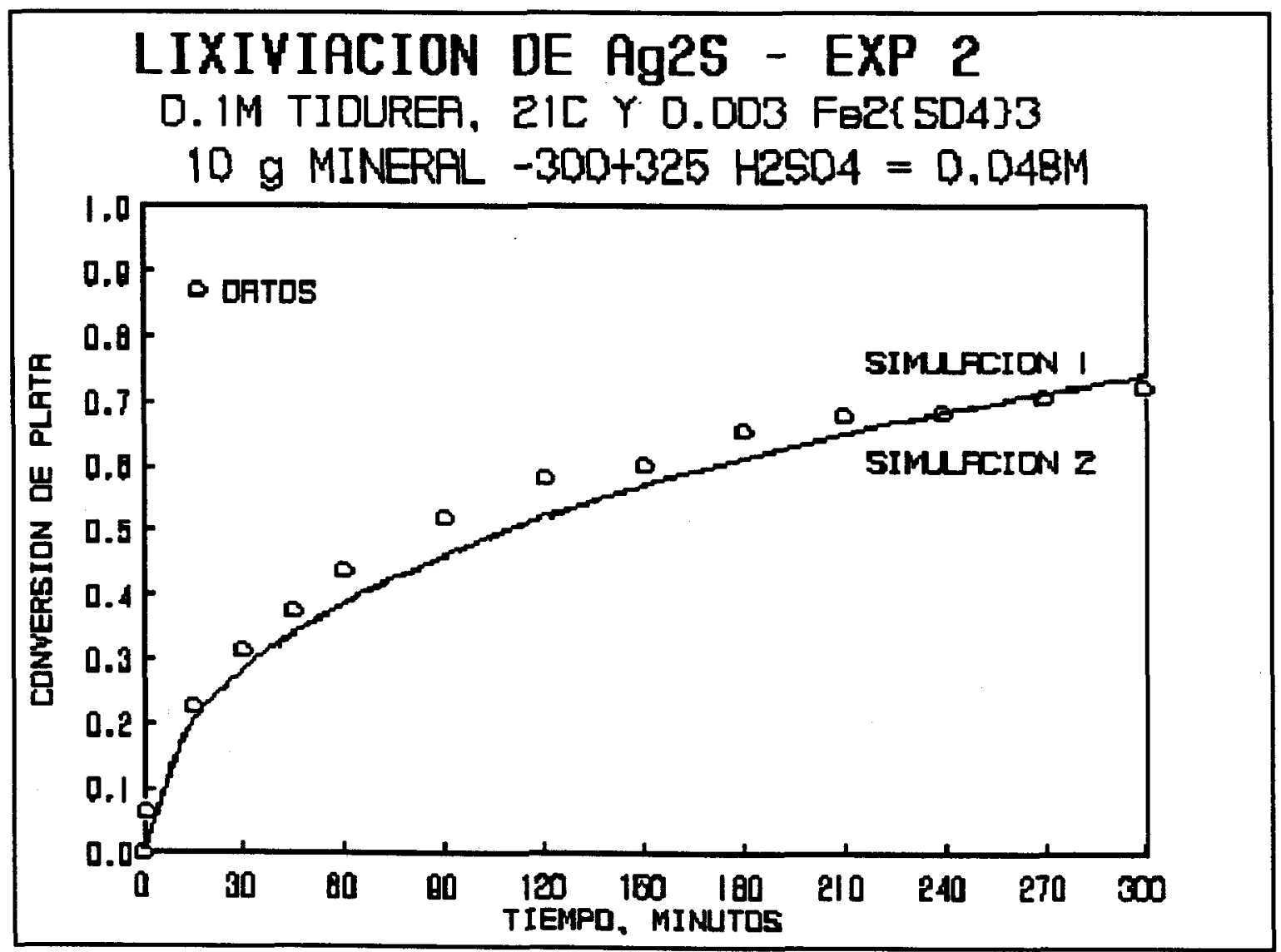




\section{Apéndice D - Experimento 4}

\section{EXPERIMENTO 4}

CONDICIONES :

$$
\begin{aligned}
& \mathrm{Fe}_{2}\left(\mathrm{SO}_{4}\right)_{3}=0.003 \mathrm{M} \\
& \mathrm{CS}\left(\mathrm{NH}_{2}\right)_{2}=0.30 \mathrm{M} \\
& \mathrm{H}_{2} \mathrm{SO}_{4}=0.048 \mathrm{M}(\mathrm{pH}=1.38) \\
& \mathrm{MINERAL}=10 \mathrm{~g}(-300+325) \\
& \text { SOLUCION }=750 \mathrm{ml}
\end{aligned}
$$

\begin{tabular}{|c|c|c|c|c|c|c|}
\hline $\begin{array}{l}\text { TIEMPO, } \\
\text { minutos }\end{array}$ & $\begin{array}{c}\text { MUESTRA, } \\
\mathrm{ml}\end{array}$ & $\begin{array}{c}\text { LECTURA, } \\
\text { Ag }\end{array}$ & $\begin{array}{l}\text { PPM } \\
\mathrm{Cu}\end{array}$ & $\begin{array}{l}\text { CONVE } \\
\text { Ag }\end{array}$ & $\begin{array}{l}\text { SION } \\
\mathrm{Cu}\end{array}$ & $\begin{array}{l}E_{h \prime} \\
m V^{\prime}\end{array}$ \\
\hline $\begin{array}{r}0.5 \\
15.0 \\
30.0 \\
45.0 \\
60.0 \\
90.0 \\
120.0 \\
145.0 \\
150.0 \\
180.0 \\
210.0 \\
240.0 \\
271.0 \\
299.0 \\
300.0\end{array}$ & $\begin{array}{l}12 . \\
6 . \\
6 . \\
6 . \\
6 . \\
6 . \\
6 . \\
6 . \\
6 . \\
6 . \\
6 . \\
6 . \\
6 . \\
6 . \\
6 .\end{array}$ & $\begin{array}{l}9.0 \\
30.0 \\
37.8 \\
38.7 \\
42.9 \\
46.2 \\
49.5 \\
51.5 \\
51.7 \\
53.9 \\
57.2 \\
58.3 \\
61.6 \\
62.7 \\
62.7\end{array}$ & $\begin{array}{l}2.2 \\
4.9 \\
5.7 \\
6.1 \\
6.2 \\
6.5 \\
6.5 \\
6.6 \\
6.6 \\
6.6 \\
6.6 \\
6.5 \\
6.7 \\
6.9 \\
6.9\end{array}$ & $\begin{array}{l}.0946 \\
.3118 \\
.3919 \\
.4010 \\
.4434 \\
.4765 \\
.5092 \\
.5289 \\
.5308 \\
.5521 \\
.5838 \\
.5942 \\
.6253 \\
.6356 \\
.6356\end{array}$ & $\begin{array}{l}.055 \\
.121 \\
.141 \\
.150 \\
.153 \\
.160 \\
.160 \\
.162 \\
.162 \\
.162 \\
.162 \\
.160 \\
.164 \\
.169 \\
.169\end{array}$ & $\begin{array}{l}-.203 \\
-.250 \\
-.252 \\
-.253 \\
-.255 \\
-.257 \\
-.257 \\
-.258 \\
-.258 \\
-.259 \\
-.260 \\
-.261 \\
-.262 \\
-.263 \\
-.263\end{array}$ \\
\hline
\end{tabular}$$
\alpha=1.8 \mathrm{e}-4 \mathrm{~min}^{-1}
$$

$\mathrm{E}_{\mathrm{h}}$ inicial (ref: sulfato de mercurio) $=-0.164 \mathrm{mV}$

RESIDUO DE $\mathrm{Ag}=2.60 \mathrm{~kg} /$ ton

RESIDUO DE Cu $=2.50 \mathrm{~kg} /$ ton

BALANCE METALURGICO DE Ag $=7.13 \mathrm{~kg} /$ ton BALANCE METALURGICO DE $\mathrm{Cu}=3.01 \mathrm{~kg} /$ ton 


\title{
Apéndice D - Experimento 4
}

\author{
LIXIVIACION DE ARGENTITA \\ CARACTERISTICAS DEL MINERAL \\ GR POR TON DE AG $=.713 E+04$ \\ DENSIDAD DEL MINERAL $=5.20$ \\ RADIO DE LA PART, $C M=.235 \mathrm{E}-02$ \\ POROSIDAD $=0.000 E+00$ \\ PROPORCION DE SOLIDO A LIQUIDO, KG/LT $=.133 \mathrm{E}-01$
}

CONSTANTES DE EQUILIBRIO A $21.00 \mathrm{C}$

CONCENTRACION INICIAL DE FE2(SO4) $3=.310 \mathrm{E}-02$

CONCENTRACION INICIAL DE $\mathrm{H} 202=0.000 \mathrm{E}+00$

CONCENTRACION INICIAL DE TIOUREA $=.300 E+00$

GAMA $=.181 \mathrm{E}-01$

VELOCIDAD DE DESCOMPOSICION DE TIOUREA = TIME* $.180 E-03$

$\begin{array}{rrrrrr}\text { FE+3 } & \text { FESO4+ } & \text { FESO4 }(\mathrm{TH})+ & \text { FEOH+2 } & \text { FE(OH) } 2+ & \text { FE2 (OH) } 2+4 \\ .463 \mathrm{E}-07 & .321 \mathrm{E}-09 & .620 \mathrm{E}-02 & .184 \mathrm{E}-08 & .105 \mathrm{E}-10 & .112 \mathrm{E}-14 \\ .0000 & .0000 & 1.0002 & .0000 & .0000 & .0000\end{array}$

FUERZA IONICA DE IA SOLUTION $=.090$

CANTIDAD DE ION FERRICO UTILIZABLE $=.620 \mathrm{E}-02$

DIFUSIVIDAD EFECTIVA DE FE+3 $=1.248922632244 \mathrm{E}-9$

ION FERRICO $=.290 \mathrm{E}-10$

SULFATO LIBRE $=.173 \mathrm{E}-01$

TIOUREA LIBRE $=.294 \mathrm{E}+00$

TOTAL DE ACIDO ANADIDO $=.47 \mathrm{E}-01$

FADS $=.310 \mathrm{E}-02$

OXIDANTE CONSUMIDO POR COBRE $=.106 \mathrm{E}-03$

\section{PERFILES ADIMENSIONALES}

TIEMPO DE AJUSTE $=180$.

Ag2T6+2 $=.002983844704287$ LIMITE $=.00009058849817237$

PRECIPITACION DE Ag2SO4.3Tu.H2O

DIFUSUVIDAD EFECTIVA $=1.075400778597 \mathrm{E}-9$

$\mathrm{Fe}^{+3}$

PH REAL $=1.382742360881$

$\begin{array}{lllll}\mathrm{Fe}^{+3} & .840 \mathrm{E}-10 & .187 \mathrm{E}-08 & .400 \mathrm{E}-08 & .462 \mathrm{E}-08 \\ \mathrm{SO}_{4}{ }^{2-} & .308 \mathrm{E}+01 & .301 \mathrm{E}+01 & .284 \mathrm{E}+01 & .279 \mathrm{E}+01 \\ \mathrm{H}^{+} & .943 \mathrm{E}+01 & .971 \mathrm{E}+01 & .104 \mathrm{E}+02 & .107 \mathrm{E}+02 \\ \mathrm{Tu}^{2+} & .464 \mathrm{E}+02 & .466 \mathrm{E}+02 & .472 \mathrm{E}+02 & .475 \mathrm{E}+02 \\ \mathrm{Fe}^{2+} & .256 \mathrm{E}+00 & .261 \mathrm{E}+00 & .271 \mathrm{E}+00 & .275 \mathrm{E}+00 \\ \mathrm{AgTu}_{2}{ }^{+} & .112 \mathrm{E}-01 & .950 \mathrm{E}-02 & .343 \mathrm{E}-02 & .587 \mathrm{E}-05\end{array}$




\section{Apéndice D - Experimento 4}

$\begin{array}{llcrrrrrr} & \text { AG+ } & \text { AGT+ } & \text { AGT2+ } & \text { AGT3+ } & \text { AGT4+ } & \text { AG2T3+2 } & \text { AG2T6+2 } \\ \text { EN IA SUPER } & .1 \mathrm{E}-13 & .2 \mathrm{E}-06 & .7 \mathrm{E}-04 & .3 \mathrm{E}-02 & .9 \mathrm{E}-03 & .2 \mathrm{E}-02 & .16 \mathrm{E}-02 \\ \text { EN SOLUCION } & .6 \mathrm{E}-17 & .8 \mathrm{E}-10 & .4 \mathrm{E}-07 & .02 \mathrm{E}-05 & .5 \mathrm{E}-06 & .4 \mathrm{E}-09 & .46 \mathrm{E}-09\end{array}$

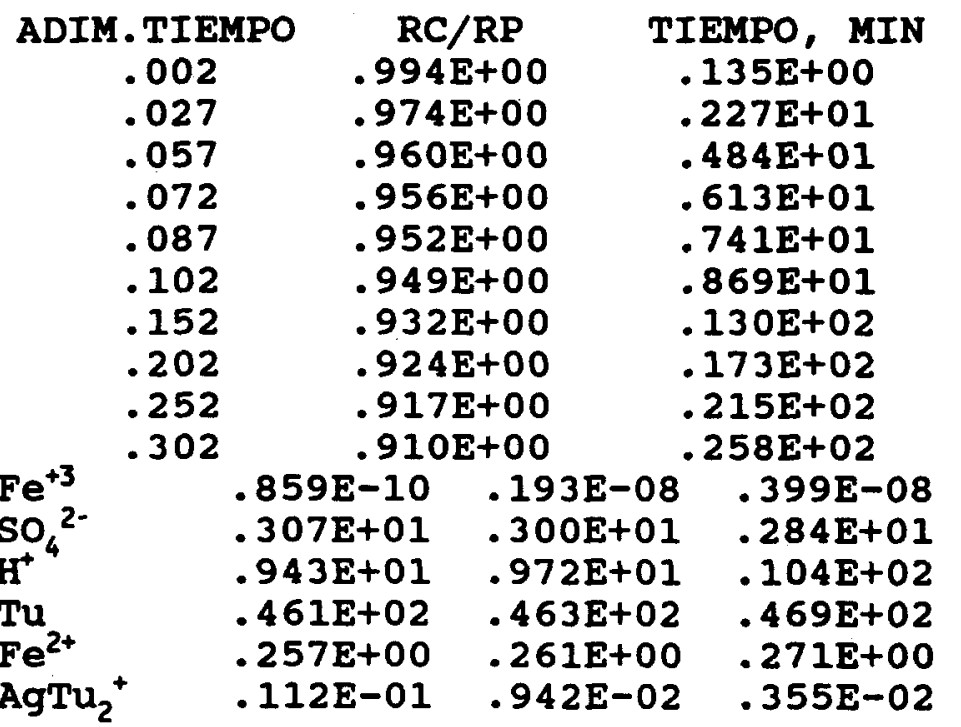

CONVERSION

$.165 \mathrm{E}-01$

$.701 \mathrm{E}-01$

$.102 \mathrm{E}+00$

$.115 \mathrm{E}+00$

$.127 \mathrm{E}+00$

$.138 \mathrm{E}+00$

$.167 \mathrm{E}+00$

$.191 \mathrm{E}+00$

$.213 \mathrm{E}+00$

$.232 E+00$

$.455 \mathrm{E}-08$

$.280 E+01$

$.106 \mathrm{E}+02$

$.471 \mathrm{E}+02$

$274 \mathrm{E}+00$

$.581 \mathrm{E}-03$
PH NUEVO

1.38

1.38

1.38

1.38

1.38

1.38

1.38

1.38

1.38

1.38

AG+ AGT+ AGT2+ AGT3+ AGT4+ AG2T3+2 AG2T6+2

EN IA SUPER .1E-13 .2E-06 .7E-04 $\quad .3 \mathrm{E}-02 \quad .9 \mathrm{E}-03 \quad .2 \mathrm{E}-02 \quad .16 \mathrm{E}-02$

EN SOLUCION .6E-15 .8E-08 .4E-05 .1E-03 $.5 \mathrm{E}-04 \quad .4 \mathrm{E}-05.44 \mathrm{E}-05$ Fes04Tu $=.73 \mathrm{E}-07$

$\begin{array}{ccccl}\text { ADIM.TIEMPO } & \text { RC/RP } & \text { TIEMPO, MIN } & \text { CONVERSION } & \text { PH NUEVO } \\ .452 & .886 \mathrm{E}+00 & .386 \mathrm{E}+02 & .280 \mathrm{E}+00 & 1.38 \\ .552 & .876 \mathrm{E}+00 & .472 \mathrm{E}+02 & .308 \mathrm{E}+00 & 1.38 \\ .652 & .866 \mathrm{E}+00 & .558 \mathrm{E}+02 & .332 \mathrm{E}+00 & 1.38 \\ .752 & .856 \mathrm{E}+00 & .643 \mathrm{E}+02 & .354 \mathrm{E}+00 & 1.38 \\ .852 & .848 \mathrm{E}+00 & .729 \mathrm{E}+02 & .375 \mathrm{E}+00 & 1.38 \\ 1.002 & .832 \mathrm{E}+00 & .857 \mathrm{E}+02 & .403 \mathrm{E}+00 & 1.38 \\ 1.152 & .820 \mathrm{E}+00 & .986 \mathrm{E}+02 & .428 \mathrm{E}+00 & 1.38 \\ 1.302 & .809 \mathrm{E}+00 & .111 \mathrm{E}+03 & .452 \mathrm{E}+00 & 1.38 \\ 1.452 & .799 \mathrm{E}+00 & .124 \mathrm{E}+03 & .474 \mathrm{E}+00 & 1.39 \\ 1.602 & .789 \mathrm{E}+00 & .137 \mathrm{E}+03 & .494 \mathrm{E}+00 & 1.39 \\ 1.902 & .762 \mathrm{E}+00 & .163 \mathrm{E}+03 & .530 \mathrm{E}+00 & 1.39 \\ 2.202 & .744 \mathrm{E}+00 & .188 \mathrm{E}+03 & .563 \mathrm{E}+00 & 1.39 \\ 2.502 & .728 \mathrm{E}+00 & .214 \mathrm{E}+03 & .592 \mathrm{E}+00 & 1.39 \\ 2.802 & .712 \mathrm{E}+00 & .240 \mathrm{E}+03 & .619 \mathrm{E}+00 & 1.39 \\ 3.102 & .696 \mathrm{E}+00 & .265 \mathrm{E}+03 & .644 \mathrm{E}+00 & 1.39 \\ 3.602 & .663 \mathrm{E}+00 & .308 \mathrm{E}+03 & .681 \mathrm{E}+00 & 1.39 \\ 4.102 & .640 \mathrm{E}+00 & .351 \mathrm{E}+03 & .714 \mathrm{E}+00 & 1.39 \\ 4.602 & .617 \mathrm{E}+00 & .394 \mathrm{E}+03 & .743 \mathrm{E}+00 & 1.39\end{array}$




\section{Apéndice D - Experimento 4}

\begin{tabular}{|c|c|c|c|c|c|c|c|c|}
\hline $\begin{array}{l}\mathrm{Fe}^{+3} \\
\mathrm{SO}_{4}^{2-} \\
\mathrm{H}^{+4} \\
\mathrm{Tu}^{2+} \\
\mathrm{Fe}^{2+} \\
\mathrm{AgTu}_{2}^{+}\end{array}$ & $\begin{array}{l}.10 \\
.30 \\
.94 \\
.42 \\
.25 \\
.11\end{array}$ & $\begin{array}{l}.595 E \\
E-09 \\
E+01 \\
E+01 \\
E+02 \\
E+00 \\
E-01\end{array}$ & $\begin{array}{l}+00 \\
.242 \mathrm{E}-08 \\
.298 \mathrm{E}+01 \\
.980 \mathrm{E}+01 \\
.423 \mathrm{E}+02 \\
.262 \mathrm{E}+00 \\
.919 \mathrm{E}-02\end{array}$ & $\begin{array}{l}37 \mathrm{E}+03 \\
.435 \mathrm{E}-08 \\
.284 \mathrm{E}+01 \\
.104 \mathrm{E}+02 \\
.428 \mathrm{E}+02 \\
.271 \mathrm{E}+00 \\
.364 \mathrm{E}-02\end{array}$ & \multicolumn{2}{|c|}{$\begin{array}{l}.770 \mathrm{E}+00 \\
.475 \mathrm{E}-08 \\
.281 \mathrm{E}+01 \\
.106 \mathrm{E}+02 \\
.429 \mathrm{E}+02 \\
.273 \mathrm{E}+00 \\
.175 \mathrm{E}-02\end{array}$} & 1.39 & \\
\hline $\begin{array}{ll}\text { EN LA SUP } \\
\text { EN SOLUCI } \\
\text { FeSO4T }\end{array}$ & $\begin{array}{l}\text { ER } \\
\text { ON } \\
u=\end{array}$ & $\begin{array}{r}\text { AG+ } \\
.1 E-13 \\
.2 E-14 \\
.82 E\end{array}$ & $\begin{array}{r}\text { AGT+ } \\
.2 \mathrm{E}-06 \\
.3 \mathrm{E}-07 \\
07\end{array}$ & $\begin{array}{l}\text { AGT2+ } \\
.7 E-04 \\
.1 E-04\end{array}$ & $\begin{array}{r}\text { AGT3+ } \\
.3 \mathrm{E}-02 \\
.4 \mathrm{E}-03\end{array}$ & $\begin{array}{r}\text { AGT4+ } \\
.8 E-03 \\
.1 E-03\end{array}$ & $\begin{array}{r}\text { AG2T3+2 } \\
.2 \mathrm{E}-02 \\
.4 \mathrm{E}-04\end{array}$ & $\begin{array}{l}\text { AG2T6+2 } \\
.14 E-02 \\
.33 E-04\end{array}$ \\
\hline
\end{tabular}

$\begin{array}{ccccc}\text { ADIM.TIEMPO } & \text { RC/RP } & \text { TIEMPO, MIN } & \text { CONVERSION } & \text { PH NUEVO } \\ 7.102 & .475 \mathrm{E}+00 & .608 \mathrm{E}+03 & .854 \mathrm{E}+00 & 1.39 \\ 8.602 & .411 \mathrm{E}+00 & .736 \mathrm{E}+03 & .901 \mathrm{E}+00 & 1.39 \\ 10.102 & .343 \mathrm{E}+00 & .865 \mathrm{E}+03 & .938 \mathrm{E}+00 & 1.39 \\ 11.602 & .267 \mathrm{E}+00 & .993 \mathrm{E}+03 & .966 \mathrm{E}+00 & 1.39 \\ 13.102 & .169 \mathrm{E}+00 & .112 \mathrm{E}+04 & .987 \mathrm{E}+00 & 1.39\end{array}$

$\begin{array}{lllll}\mathrm{Fe}^{+3} & .143 \mathrm{E}-09 & .563 \mathrm{E}-08 & .577 \mathrm{E}-08 & .536 \mathrm{E}-08 \\ \mathrm{SO}_{4}^{2-} & .309 \mathrm{E}+01 & .281 \mathrm{E}+01 & .279 \mathrm{E}+01 & .281 \mathrm{E}+01 \\ \mathrm{H}^{+} & .936 \mathrm{E}+01 & .106 \mathrm{E}+02 & .106 \mathrm{E}+02 & .105 \mathrm{E}+02 \\ \mathrm{Tu}^{+} & .363 \mathrm{E}+02 & .371 \mathrm{E}+02 & .372 \mathrm{E}+02 & .370 \mathrm{E}+02 \\ \mathrm{Fe}^{2+} & .256 \mathrm{E}+00 & .274 \mathrm{E}+00 & .275 \mathrm{E}+00 & .273 \mathrm{E}+00 \\ \mathrm{AgTu}_{2}{ }^{+} & .124 \mathrm{E}-01 & .224 \mathrm{E}-02 & .248 \mathrm{E}-03 & .233 \mathrm{E}-02\end{array}$

$\begin{array}{llcrrrrrr} & \text { AGt } & \text { AGT+ } & \text { AGT2+ } & \text { AGT3+ } & \text { AGT4+ } & \text { AG2T3+2 } & \text { AG2T6+2 } \\ \text { EN LA SUPER } & .2 \mathrm{E}-13 & .2 \mathrm{E}-06 & .8 \mathrm{E}-04 & .2 \mathrm{E}-02 & .6 \mathrm{E}-03 & .3 \mathrm{E}-02 & .12 \mathrm{E}-02 \\ \text { EN SOLUCION } & .4 \mathrm{E}-14 & .4 \mathrm{E}-07 & .1 \mathrm{E}-04 & .5 \mathrm{E}-03 & .1 \mathrm{E}-03 & .9 \mathrm{E}-04 & .44 \mathrm{E}-04 \\ \text { FeSO4Tu }= & .98 \mathrm{E}-07 & & & & & & \end{array}$

DIFUSIVIDAD EFECTIVA DE FE+3 $=1.075400778597 \mathrm{E}-9$

$\mathrm{CONV}=.17 \mathrm{E}-01 * t+-.16 \mathrm{E}-03 * t * * 2+.26 \mathrm{E}-06 * t * * 3$ 
Apéndice D - Experimento 4

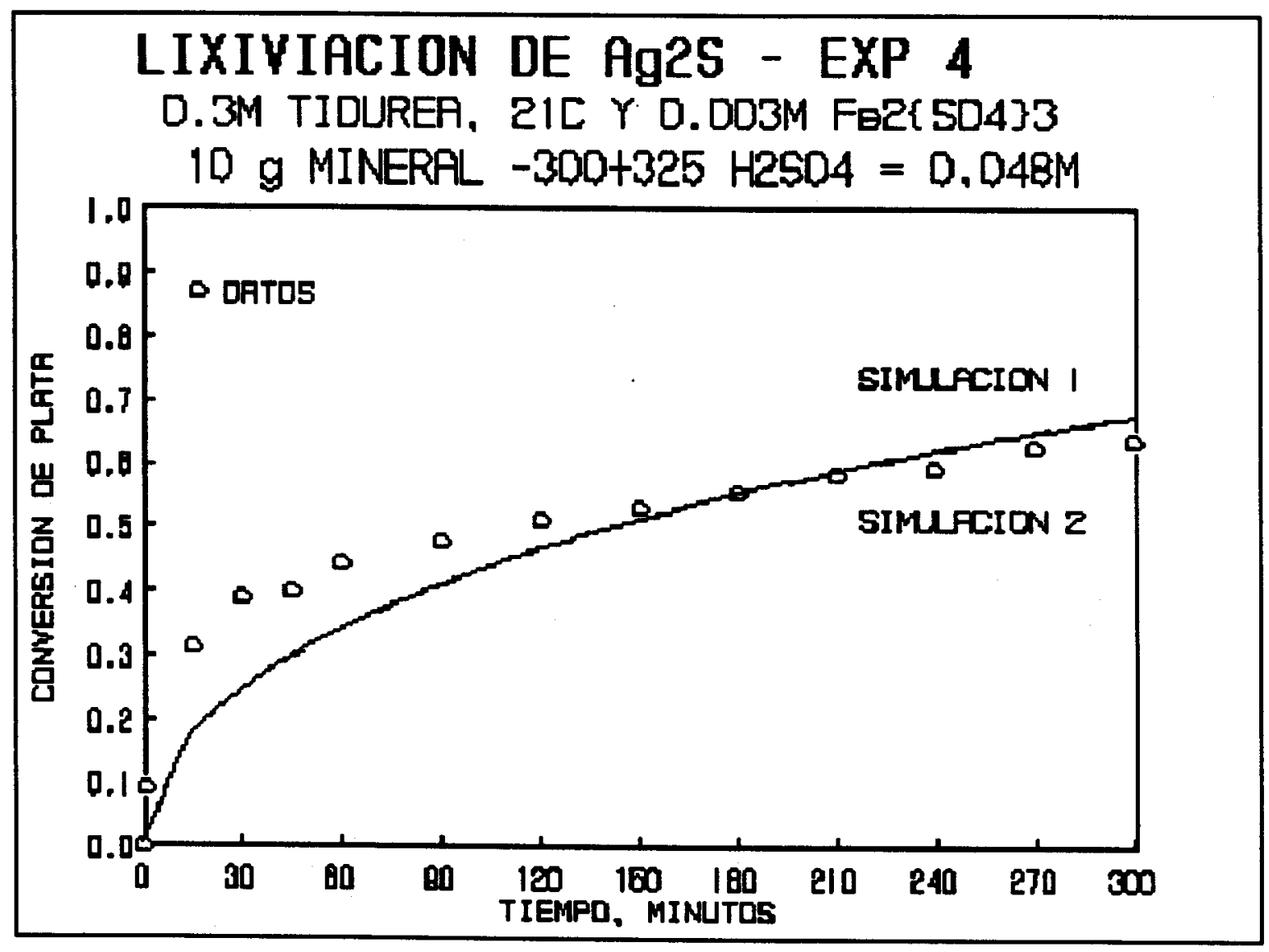




\section{Apéndice D - Experimento 5}

\section{EXPERIMENTO 5}

CONDICIONES :

$$
\begin{aligned}
& \mathrm{Fe}_{2}\left(\mathrm{SO}_{4}\right)_{3}=0.003 \mathrm{M} \\
& \mathrm{CS}^{2}\left(\mathrm{NH}_{2}\right)_{2}=0.10 \mathrm{M} \\
& \mathrm{H}_{2} \mathrm{SO}_{4}=0.048 \mathrm{M}(\mathrm{pH}=1.38) \\
& \mathrm{MINERAL}=30 \mathrm{~g}(-300+325) \\
& \text { SOLUCION }=750 \mathrm{ml} \\
& \alpha=2.3 \mathrm{e}-4 \mathrm{~min}^{-1} \\
& \mathrm{E}_{\mathrm{h}} \text { inicial (ref: sulfato de mercurio) }=-0.130 \mathrm{mV}
\end{aligned}
$$

\begin{tabular}{|c|c|c|c|c|c|c|}
\hline $\begin{array}{l}\text { TIEMPO, } \\
\text { minutos }\end{array}$ & $\begin{array}{c}\text { MUESTRA, } \\
\text { ml }\end{array}$ & $\begin{array}{l}\text { LECTURA } \\
\text { Ag }\end{array}$ & $\begin{array}{l}\mathrm{PPM} \\
\mathrm{Cu}\end{array}$ & $\begin{array}{c}\text { CONV } \\
\text { Ag }\end{array}$ & $\begin{array}{l}\text { ION } \\
\mathrm{Cu}\end{array}$ & $\begin{array}{l}E_{h h^{\prime}} \\
m V^{\prime}\end{array}$ \\
\hline $\begin{array}{r}1.5 \\
15.0 \\
30.0 \\
45.0 \\
60.0 \\
90.0 \\
120.0 \\
145.0 \\
150.0 \\
180.0 \\
210.0 \\
240.0 \\
270.0 \\
295.0 \\
300.0\end{array}$ & $\begin{array}{l}12 . \\
6 . \\
6 . \\
6 . \\
6 . \\
6 . \\
6 . \\
6 . \\
6 . \\
6 . \\
6 . \\
6 . \\
6 . \\
6 . \\
6 .\end{array}$ & $\begin{array}{r}16.5 \\
51.5 \\
68.4 \\
81.0 \\
88.0 \\
108.9 \\
129.3 \\
134.0 \\
134.8 \\
154.0 \\
162.3 \\
170.5 \\
184.4 \\
190.0 \\
190.7\end{array}$ & $\begin{array}{l}5.0 \\
11.2 \\
13.7 \\
15.4 \\
16.1 \\
17.9 \\
18.9 \\
19.2 \\
19.3 \\
20.0 \\
20.7 \\
21.0 \\
21.7 \\
21.9 \\
22.0\end{array}$ & $\begin{array}{l}.0562 \\
.1737 \\
.2299 \\
.2715 \\
.2944 \\
.3622 \\
.4279 \\
.4429 \\
.4454 \\
.5056 \\
.5314 \\
.5567 \\
.5991 \\
.6161 \\
.6182\end{array}$ & $\begin{array}{l}.044 \\
.098 \\
.119 \\
.134 \\
.140 \\
.155 \\
.163 \\
.166 \\
.167 \\
.172 \\
.178 \\
.180 \\
.186 \\
.187 \\
.188\end{array}$ & $\begin{array}{l}-.218 \\
-.219 \\
-.220 \\
-.221 \\
-.222 \\
-.223 \\
-.225 \\
-.226 \\
-.226 \\
-.227 \\
-.227 \\
-.228 \\
-.229 \\
-.230 \\
-.230\end{array}$ \\
\hline
\end{tabular}

RESIDUO DE Ag $=2.80 \mathrm{~kg} / \mathrm{ton}$

RESIDUO DE Cu $=2.30 \mathrm{~kg} /$ ton

BALANCE METALURGICO DE Ag $=7.33 \mathrm{~kg} /$ ton

BALANCE METALURGICO DE $\mathrm{Cu}=2.83 \mathrm{~kg} /$ ton 


\section{Apéndice D - Experimento 5}

\section{LIXIVIACION DE ARGENTITA}

CARACTERISTICAS DEL MINERAL

GR POR TON DE AG $=.733 \mathrm{E}+04$

DENSIDAD DEL MINERAL $=5.20$

RADIO DE LA PART, CM = .235E-02

POROSIDAD $=0.000 E+00$

PROPORCION DE SOLIDO A LIQUIDO, KG/LT $=.400 E-01$

CONSTANTES DE EQUILIBRIO A $21.00 \mathrm{C}$

CONCENTRACION INICIAL DE FE2(SO4) $3=.310 E-02$

CONCENTRACION INICIAL DE $\mathrm{H} 2 \mathrm{O2}=0.000 \mathrm{E}+00$

CONCENTRACION INICIAL DE TIOUREA $=.100 \mathrm{E}+00$

GAMA $=\quad .176 \mathrm{E}-01$

VELOCIDAD DE DESCOMPOSICION DE TIOUREA = TIME* .232E-03

$\begin{array}{rrrrrr}\text { FE+3 } & \text { FESO4+ } & \text { FESO4 }(\mathrm{TH})+ & \text { FEOH+2 } & \text { FE(OH) } 2+ & \text { FE2 (OH) } 2+4 \\ .145 E-06 & .100 E-08 & .620 \mathrm{E}-02 & .578 \mathrm{E}-08 & .329 \mathrm{E}-10 & .110 \mathrm{E}-13 \\ .0000 & .0000 & 1.0002 & .0000 & .0000 & .0000\end{array}$

FUERZA IONICA DE LA SOLUTION $=.090$

CANTIDAD DE ION FERRICO UTILIZABLE $=.620 \mathrm{E}-02$

DIFUSIVIDAD EFECTIVA DE FE+3 = 1.319971107908E-9

ION FERRICO $=.907 \mathrm{E}-10$

SULFATO LIBRE $=.173 \mathrm{E}-01$

TIOUREA LIBRE $=.938 \mathrm{E}-01$

TOTAL DE ACIDO ANADIDO $=\quad .47 \mathrm{E}-01$

FADS $=.310 \mathrm{E}-02$

OXIDANTE CONSUMIDO POR COBRE $=.335 \mathrm{E}-03$

\section{PERFILES ADIMENSIONALES}

TIEMPO DE AJUSTE $=120$.

Ag2T6+2 $=.0001777900454878$ LIMITE $=.00004634386259265$

PRECIPITACION DE Ag2SO4.3Tu.H2O

DIFUSUVIDAD EFECTIVA $=1.12268341361 \mathrm{E}-9$

\begin{tabular}{lcccc}
\multicolumn{1}{c}{$\mathrm{PH}$} & $\mathrm{REAL}=1.382742224771$ \\
$\mathrm{Fe}^{+3}$ & $.243 \mathrm{E}-08$ & $.598 \mathrm{E}-08$ & $.121 \mathrm{E}-07$ & $.140 \mathrm{E}-07$ \\
$\mathrm{SO}_{4}{ }^{2-}$ & $.312 \mathrm{E}+01$ & $.305 \mathrm{E}+01$ & $.285 \mathrm{E}+01$ & $.279 \mathrm{E}+01$ \\
$\mathrm{H}^{+}$ & $.924 \mathrm{E}+01$ & $.954 \mathrm{E}+01$ & $.104 \mathrm{E}+02$ & $.107 \mathrm{E}+02$ \\
$\mathrm{Tu}$ & $.150 \mathrm{E}+02$ & $.150 \mathrm{E}+02$ & $.153 \mathrm{E}+02$ & $.154 \mathrm{E}+02$ \\
$\mathrm{Fe}^{2+}$ & $.254 \mathrm{E}+00$ & $.258 \mathrm{E}+00$ & $.271 \mathrm{E}+00$ & $.275 \mathrm{E}+00$ \\
$\mathrm{AgTu}_{2}{ }^{+}$ & $.113 \mathrm{E}-01$ & $.995 \mathrm{E}-02$ & $.470 \mathrm{E}-02$ & $.581 \mathrm{E}-04$
\end{tabular}


Apéndice D - Experimento 5

$\begin{array}{llcrrrrrr} & & \text { AGt } & \text { AGT+ } & \text { AGT2+ } & \text { AGT3+ } & \text { AGT4+ } & \text { AG2T3+2 } & \text { AG2T6+2 } \\ \text { EN LA SUPER } & .1 \mathrm{E}-12 & .5 \mathrm{E}-06 & .7 \mathrm{E}-04 & .9 \mathrm{E}-03 & .1 \mathrm{E}-03 & .5 \mathrm{E}-02 & .17 \mathrm{E}-03 \\ \text { EN SOLUCION } & .5 \mathrm{E}-15 & .3 \mathrm{E}-08 & .4 \mathrm{E}-06 & .5 \mathrm{E}-05 & .5 \mathrm{E}-06 & .1 \mathrm{E}-06 & .47 \mathrm{E}-08\end{array}$

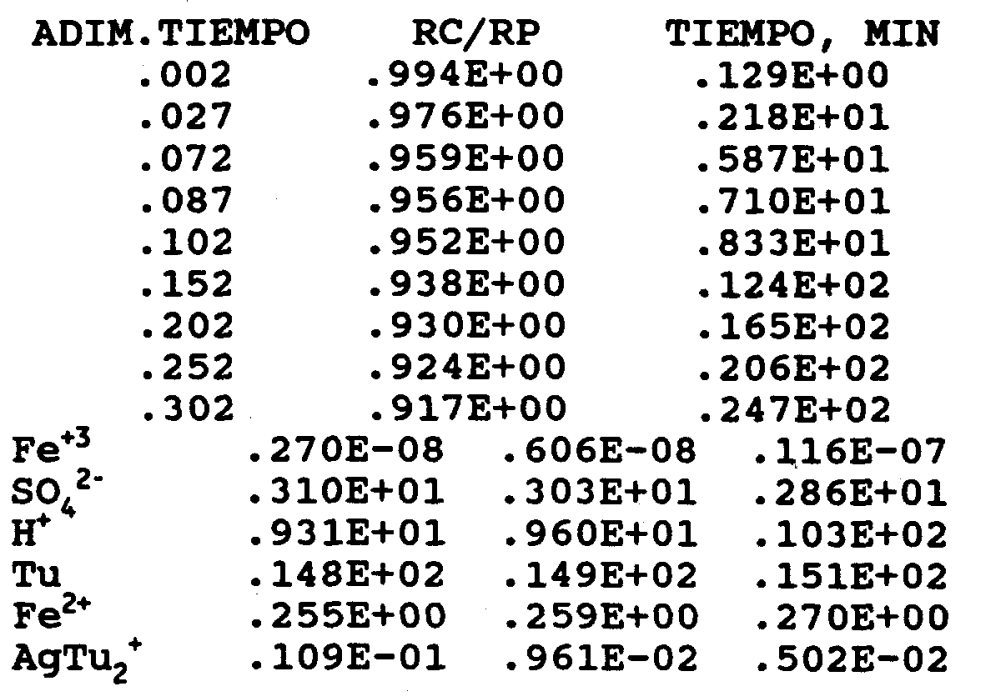

CONVERSION

$.156 \mathrm{E}-01$

$.659 \mathrm{E}-01$

$.108 \mathrm{E}+00$

$.119 \mathrm{E}+00$

$.128 \mathrm{E}+00$

$.155 \mathrm{E}+00$

$.177 \mathrm{E}+00$

$.197 \mathrm{E}+00$

$.214 \mathrm{E}+00$

.133E-07

$.281 \mathrm{E}+01$

$.106 \mathrm{E}+02$

$.152 \mathrm{E}+02$

$.274 \mathrm{E}+00$

$.221 \mathrm{E}-02$
PH NUEVO

1.38

1.38

1.38

1.38

1.38

1.39

1.39

1.39

1.39

$\begin{array}{llcrrrrrr} & & \text { AG+ } & \text { AGT+ } & \text { AGT2+ } & \text { AGT3+ } & \text { AGT4+ } & \text { AG2T3+2 } & \text { AG2T6+2 } \\ \text { EN LA SUPER } & .1 \mathrm{E}-12 & .5 \mathrm{E}-06 & .7 \mathrm{E}-04 & .9 \mathrm{E}-03 & .9 \mathrm{E}-04 & .5 \mathrm{E}-02 & .16 \mathrm{E}-03 \\ \text { EN SOLUCION } & .2 \mathrm{E}-13 & .1 \mathrm{E}-06 & .1 \mathrm{E}-04 & .2 \mathrm{E}-03 & .2 \mathrm{E}-04 & .2 \mathrm{E}-03 & .66 \mathrm{E}-05\end{array}$

$\begin{array}{cc}\text { ADIM.TIEMPO } & \text { RC/RP } \\ .452 & .897 \mathrm{E}+00 \\ .552 & .888 \mathrm{E}+00 \\ .652 & .879 \mathrm{E}+00 \\ .752 & .871 \mathrm{E}+00 \\ .852 & .864 \mathrm{E}+00 \\ 1.002 & .851 \mathrm{E}+00 \\ 1.152 & .841 \mathrm{E}+00 \\ 1.302 & .832 \mathrm{E}+00 \\ 1.452 & .824 \mathrm{E}+00 \\ 1.602 & .816 \mathrm{E}+00 \\ 1.902 & .795 \mathrm{E}+00 \\ 2.202 & .781 \mathrm{E}+00 \\ 2.502 & .769 \mathrm{E}+00 \\ 2.802 & .757 \mathrm{E}+00 \\ 3.102 & .746 \mathrm{E}+00 \\ 3.602 & .723 \mathrm{E}+00 \\ 4.102 & .707 \mathrm{E}+00 \\ 4.602 & .693 \mathrm{E}+00 \\ 5.102 & .680 \mathrm{E}+00\end{array}$

PH NUEVO

1.39

1.39

1.39

1.39

1.39

1.39

1.39

1.39

1.39

1.39

1.39

1.39

1.39

1.39

1.39

1.39

1.39

1. 39

1.39 


\section{Apéndice D - Experimento 5}

$\begin{array}{lllll}\mathrm{Fe}^{+3} & .598 \mathrm{E}-08 & .822 \mathrm{E}-08 & .119 \mathrm{E}-07 & .128 \mathrm{E}-07 \\ \mathrm{SO}^{2-} & .301 \mathrm{E}+01 & .296 \mathrm{E}+01 & .286 \mathrm{E}+01 & .284 \mathrm{E}+01 \\ \mathrm{H}^{+} & .964 \mathrm{E}+01 & .986 \mathrm{E}+01 & .103 \mathrm{E}+02 & .104 \mathrm{E}+02 \\ \mathrm{Tu}^{2+} & .132 \mathrm{E}+02 & .132 \mathrm{E}+02 & .134 \mathrm{E}+02 & .134 \mathrm{E}+02 \\ \mathrm{Fe}^{2+} & .260 \mathrm{E}+00 & .263 \mathrm{E}+00 & .270 \mathrm{E}+00 & .271 \mathrm{E}+00 \\ \mathrm{AgTu}_{2}{ }^{+} & .887 \mathrm{E}-02 & .777 \mathrm{E}-02 & .503 \mathrm{E}-02 & .402 \mathrm{E}-02\end{array}$

AGt AGT + AGT2+ AGT3+ AGT4+ AG2T3+2 AG2T6+2

EN LA SUPER .1E-12 .4E-06 .5E-04 $.6 \mathrm{E}-03 \quad .6 \mathrm{E}-04 \quad .4 \mathrm{E}-02 \quad .81 \mathrm{E}-04$

EN SOLUCION .5E-13 .2E-06 .2E-04 $.3 \mathrm{E}-03 \quad .3 \mathrm{E}-04 \quad .7 \mathrm{E}-03 \quad .17 \mathrm{E}-04$

FeSO4Tu $=.14 \mathrm{E}-05$ 
Apéndice D - Experimento 5

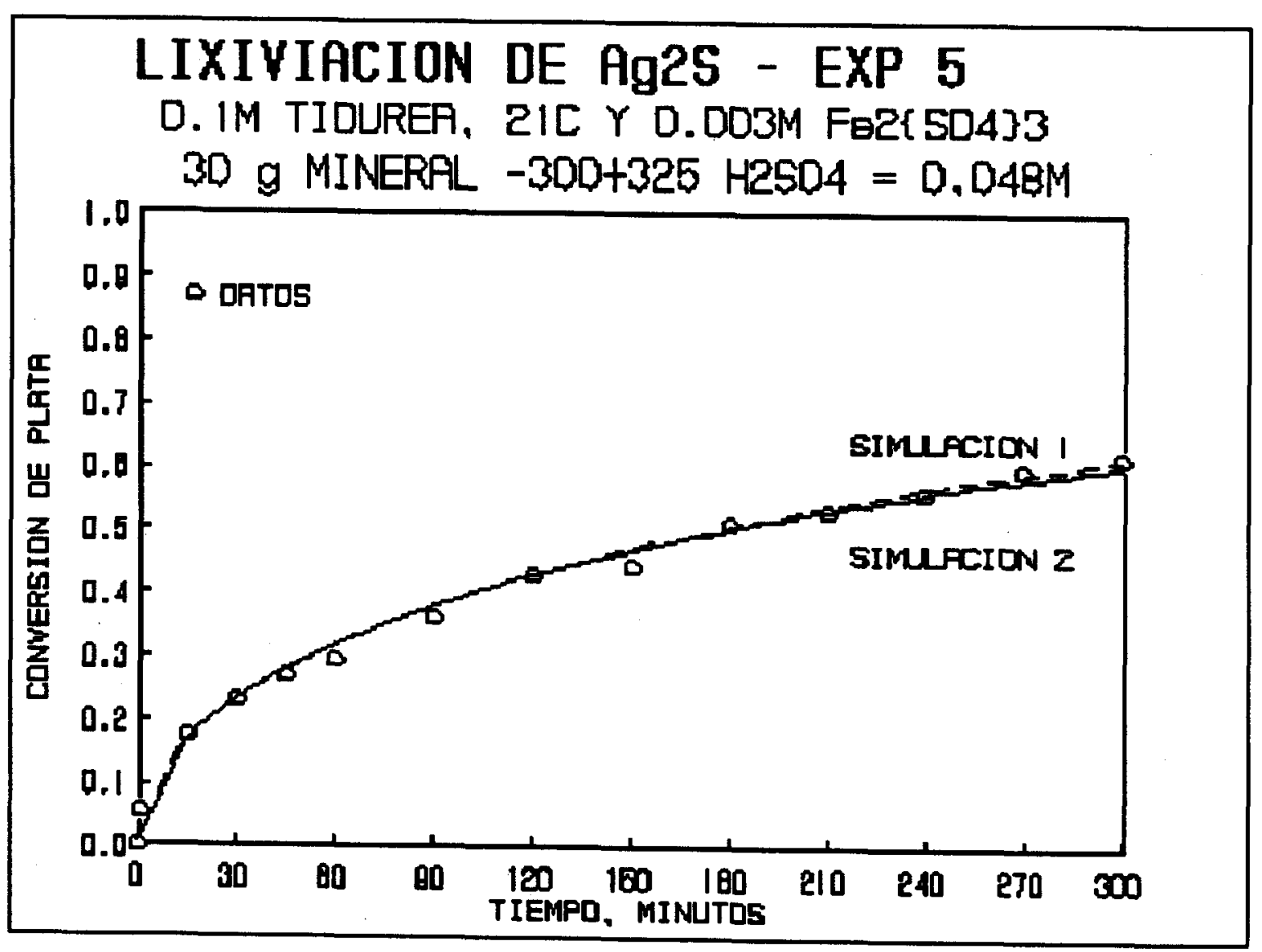




\section{Apéndice D - Experimento 6}

\section{EXPERIMENTO 6}

\section{CONDICIONES :}

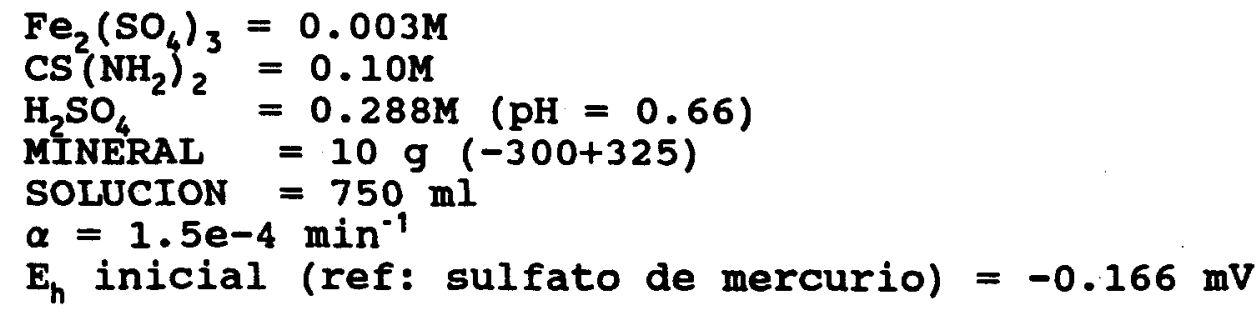

\begin{tabular}{rcccccc} 
TIEMPO, & MUESTRA, & \multicolumn{2}{c}{ LECTURA, PPM } & \multicolumn{2}{c}{ CONVERSION } & E $_{h \prime}$ \\
minutos & $\mathrm{ml}$ & $\mathrm{Ag}$ & $\mathrm{Cu}$ & $\mathrm{Ag}$ & $\mathrm{Cu}$ & $\mathrm{mV}$ \\
1.0 & 12. & 6.5 & 1.9 & .0659 & .047 & -.207 \\
15.0 & 6. & 20.0 & 4.0 & .2007 & .097 & -.256 \\
30.0 & 6. & 27.5 & 4.9 & .2750 & .119 & -.257 \\
45.0 & 6. & 35.1 & 5.5 & .3496 & .133 & -.258 \\
60.0 & 6. & 38.7 & 6.0 & .3846 & .145 & -.260 \\
90.0 & 6. & 46.2 & 6.5 & .4571 & .157 & -.262 \\
120.0 & 6. & 52.8 & 6.8 & .5203 & .164 & -.264 \\
145.0 & 6. & 55.5 & 7.1 & .5459 & .171 & -.265 \\
150.0 & 6. & 56.1 & 7.1 & .5516 & .171 & -.265 \\
180.0 & 6. & 60.5 & 7.2 & .5926 & .173 & -.266 \\
210.0 & 6. & 62.7 & 7.4 & .6130 & .177 & -.266 \\
240.0 & 6. & 66.0 & 7.4 & .6433 & .177 & -.267 \\
270.0 & 6. & 68.2 & 7.5 & .6632 & .179 & -.267 \\
297.0 & 6. & 71.3 & 7.6 & .6912 & .182 & -.268 \\
300.0 & 6. & 71.5 & 7.6 & .6930 & .182 & -.268
\end{tabular}

RESIDUO DE Ag $=2.27 \mathrm{~kg} / \mathrm{ton}$

RESIDUO DE $\mathrm{Cu}=2.50 \mathrm{~kg} /$ ton

BALANCE METALURGICO DE Ag $=7.39 \mathrm{~kg} /$ ton

BALANCE METALURGICO DE $\mathrm{Cu}=3.05 \mathrm{~kg} / \mathrm{ton}$ 


\section{Apéndice $D$ - Experimento 6}

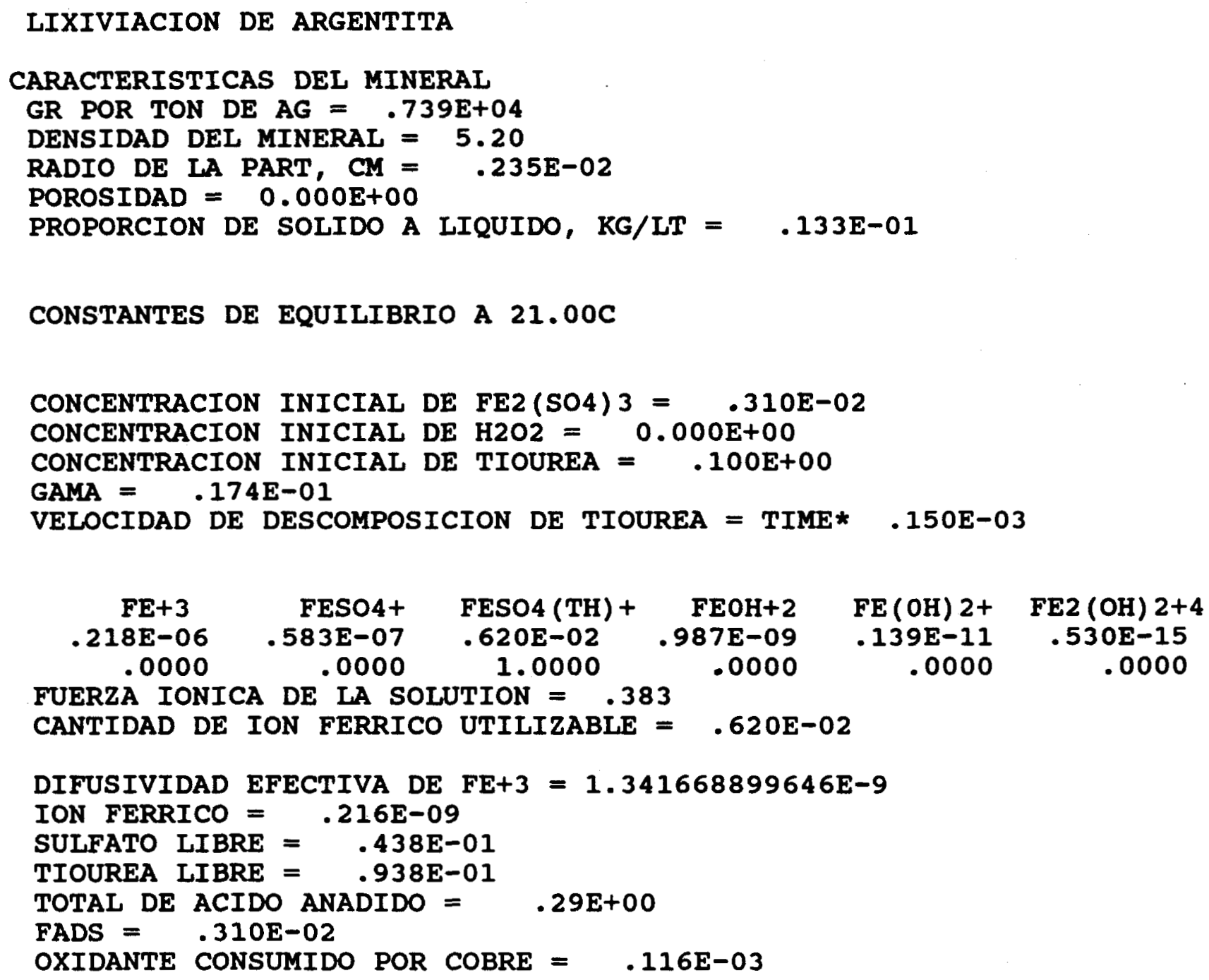

PERFILES ADIMENSIONALES

TIEMPO DE AJUSTE $=150$.

Ag2T6+2 $=.3682993751639$ LIMITE $=.000002560983872971$

PRECIPITACION DE Ag2SO4.3Tu.H2O

DIFUSUVIDAD EFECTIVA $=1.360907600524 \mathrm{E}-9$

PH REAL $=.6620714281756$

$\begin{array}{lllll}\mathrm{Fe}^{3+} & .110 \mathrm{E}-07 & .161 \mathrm{E}-07 & .299 \mathrm{E}-07 & .349 \mathrm{E}-07 \\ \mathrm{SO}_{4}{ }^{2-} & .742 \mathrm{E}+01 & .734 \mathrm{E}+01 & .714 \mathrm{E}+01 & .706 \mathrm{E}+01 \\ \mathrm{H}^{+} & .531 \mathrm{E}+02 & .532 \mathrm{E}+02 & .535 \mathrm{E}+02 & .537 \mathrm{E}+02 \\ \mathrm{Tu}^{2+} & .136 \mathrm{E}+02 & .139 \mathrm{E}+02 & .148 \mathrm{E}+02 & .151 \mathrm{E}+02 \\ \mathrm{Fe}^{2+} & .130 \mathrm{E}+00 & .130 \mathrm{E}+00 & .130 \mathrm{E}+00 & .130 \mathrm{E}+00 \\ \mathrm{AgTu}_{2}{ }^{+} & .119 \mathrm{E}-01 & .938 \mathrm{E}-02 & .252 \mathrm{E}-02 & .500 \mathrm{E}-05\end{array}$


Apéndice D - Experimento 6

$\begin{array}{llcrrrrrr} & & \text { AG+ } & \text { AGT+ } & \text { AGT2+ } & \text { AGT3+ } & \text { AGT4+ } & \text { AG2T3+2 } & \text { AG2T6+2 } \\ \text { EN LA SUPER } & .1 \mathrm{E}-12 & .6 \mathrm{E}-06 & .7 \mathrm{E}-04 & .9 \mathrm{E}-03 & .9 \mathrm{E}-04 & .6 \mathrm{E}-02 & .15 \mathrm{E}-03 \\ \text { EN SOLUCION } & .5 \mathrm{E}-16 & .2 \mathrm{E}-09 & .3 \mathrm{E}-07 & .4 \mathrm{E}-06 & .4 \mathrm{E}-07 & .1 \mathrm{E}-08 & .34 \mathrm{E}-10\end{array}$

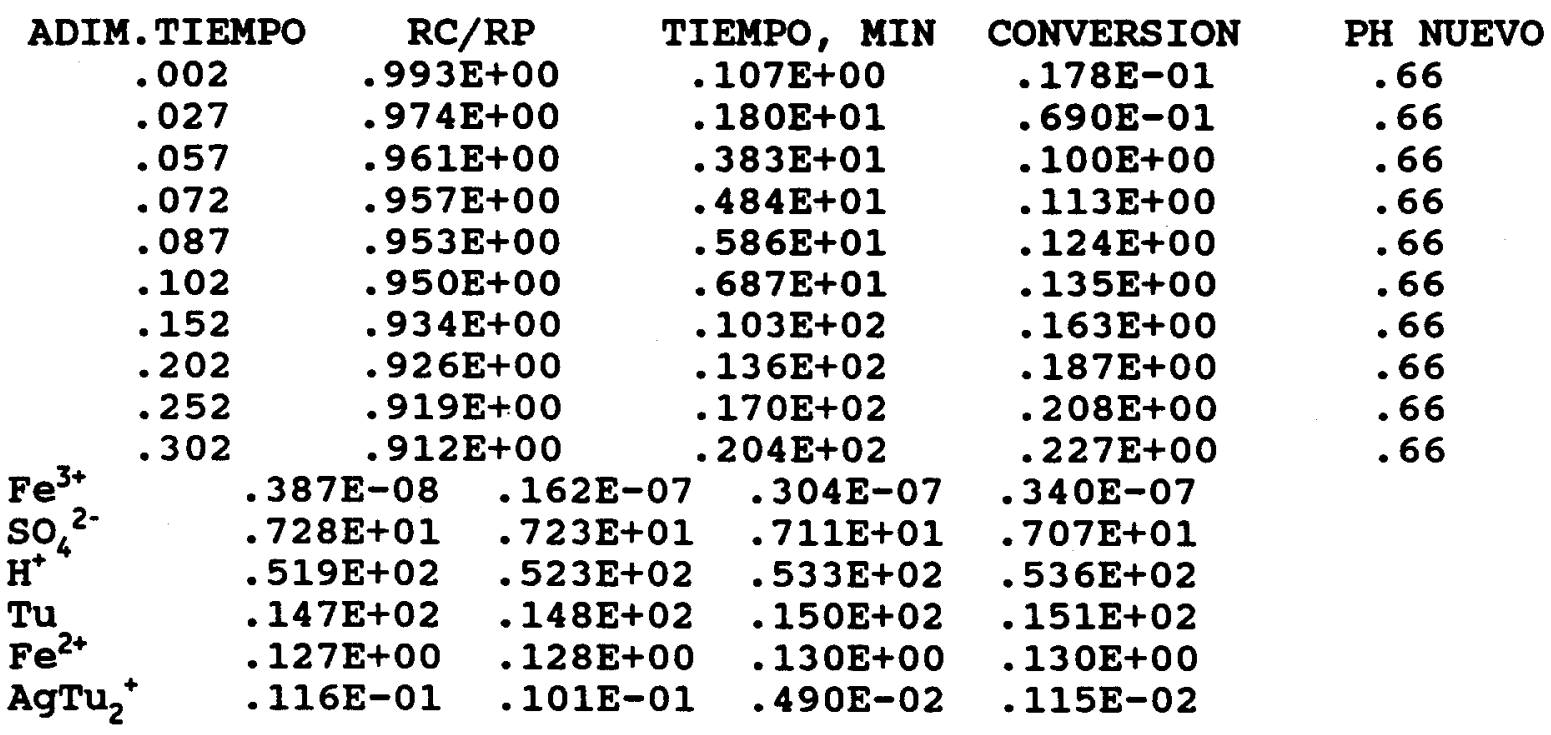

$\begin{array}{llcrrrrrr} & & \text { AG+ } & \text { AGT+ } & \text { AGT2+ } & \text { AGT3+ } & \text { AGT4+ } & \text { AG2T3+2 } & \text { AG2T6+2 } \\ \text { EN LA SUPER } & .1 \mathrm{E}-12 & .5 \mathrm{E}-06 & .7 \mathrm{E}-04 & .9 \mathrm{E}-03 & .1 \mathrm{E}-03 & .6 \mathrm{E}-02 & .17 \mathrm{E}-03 \\ \text { EN SOLUCION } & .1 \mathrm{E}-13 & .5 \mathrm{E}-07 & .7 \mathrm{E}-05 & .9 \mathrm{E}-04 & .1 \mathrm{E}-04 & .5 \mathrm{E}-04 & .18 \mathrm{E}-05 \\ \text { FeSO4Tu }= & .68 \mathrm{E}-06 & & & & & \end{array}$

$\begin{array}{cc}\text { ADIM. TIEMPO } & \text { RC/RP } \\ .452 & .889 \mathrm{E}+00 \\ .552 & .879 \mathrm{E}+00 \\ .652 & .869 \mathrm{E}+00 \\ .752 & .860 \mathrm{E}+00 \\ .852 & .852 \mathrm{E}+00 \\ 1.002 & .836 \mathrm{E}+00 \\ 1.152 & .825 \mathrm{E}+00 \\ 1.302 & .814 \mathrm{E}+00 \\ 1.452 & .804 \mathrm{E}+00 \\ 1.602 & .795 \mathrm{E}+00 \\ 1.902 & .769 \mathrm{E}+00 \\ 2.202 & .752 \mathrm{E}+00 \\ 2.502 & .736 \mathrm{E}+00 \\ 2.802 & .720 \mathrm{E}+00 \\ 3.102 & .705 \mathrm{E}+00 \\ 3.602 & .674 \mathrm{E}+00 \\ 4.102 & .651 \mathrm{E}+00 \\ 4.602 & .630 \mathrm{E}+00\end{array}$

TIEMPO, MIN

$.305 E+02$

$.373 \mathrm{E}+02$

$.441 \mathrm{E}+02$

$.508 \mathrm{E}+02$

$.576 \mathrm{E}+02$

$.677 \mathrm{E}+02$

$.779 \mathrm{E}+02$

$.880 \mathrm{E}+02$

$.982 \mathrm{E}+02$

$.108 \mathrm{E}+03$

$.129 E+03$

$.149 \mathrm{E}+03$

$.169 \mathrm{E}+03$

$.189 \mathrm{E}+03$

$.210 \mathrm{E}+03$

$.244 \mathrm{E}+03$

$.277 \mathrm{E}+03$

$.311 E+03$
CONVERSION
$.274 \mathrm{E}+00$
$.300 \mathrm{E}+00$
$.324 \mathrm{E}+00$
$.346 \mathrm{E}+00$
$.366 \mathrm{E}+00$
$.394 \mathrm{E}+00$
$.419 \mathrm{E}+00$
$.442 \mathrm{E}+00$
$.463 \mathrm{E}+00$
$.483 \mathrm{E}+00$
$.518 \mathrm{E}+00$
$.550 \mathrm{E}+00$
$.579 \mathrm{E}+00$
$.606 \mathrm{E}+00$
$.630 \mathrm{E}+00$
$.667 \mathrm{E}+00$
$.699 E+00$
$.728 \mathrm{E}+00$

PH NUEVO

.66

.66

.66

.66

.66

.66

.66

.66

.66

.66

.66

.66

.66

.66

.66

.66

.66

.66 


\section{Apéndice D - Experimento 6}

$\begin{array}{lcccc} & 5.102 & .609 \mathrm{E}+00 & .345 \mathrm{E}+03 & .755 \mathrm{E}+00 \\ \mathrm{Fe}^{3+} & .517 \mathrm{E}-08 & .195 \mathrm{E}-07 & .322 \mathrm{E}-07 & .347 \mathrm{E}-07 \\ \mathrm{SO}^{2-} & .727 \mathrm{E}+01 & .721 \mathrm{E}+01 & .710 \mathrm{E}+01 & .708 \mathrm{E}+01 \\ \mathrm{H}^{+} & .520 \mathrm{E}+02 & .525 \mathrm{E}+02 & .533 \mathrm{E}+02 & .536 \mathrm{E}+02 \\ \mathrm{Tu}^{2+} & .138 \mathrm{E}+02 & .139 \mathrm{E}+02 & .141 \mathrm{E}+02 & .141 \mathrm{E}+02 \\ \mathrm{Fe}^{2+} & .127 \mathrm{E}+00 & .128 \mathrm{E}+00 & .130 \mathrm{E}+00 & .130 \mathrm{E}+00 \\ \mathrm{AgTu}_{2}{ }^{+} & .110 \mathrm{E}-01 & .920 \mathrm{E}-02 & .445 \mathrm{E}-02 & .235 \mathrm{E}-02\end{array}$

$\begin{array}{llcrrrrrr} & & \text { AGt } & \text { AGT+ } & \text { AGT2+ } & \text { AGT3+ } & \text { AGT4+ } & \text { AG2T3+2 } & \text { AG2T6+2 } \\ \text { EN LA SUPER } & .1 \mathrm{E}-12 & .5 \mathrm{E}-06 & .7 \mathrm{E}-04 & .8 \mathrm{E}-03 & .8 \mathrm{E}-04 & .5 \mathrm{E}-02 & .14 \mathrm{E}-03 \\ \text { EN SOLUCION } & .3 \mathrm{E}-13 & .1 \mathrm{E}-06 & .1 \mathrm{E}-04 & .2 \mathrm{E}-03 & .2 \mathrm{E}-04 & .2 \mathrm{E}-03 & .65 \mathrm{E}-05 \\ \text { FeSO4Tu }= & .84 \mathrm{E}-06 & & & & & & \end{array}$

$\begin{array}{ccccc}\text { ADIM.TIEMPO } & \text { RC/RP } & \text { TIEMPO, MIN } & \text { CONVERSION } & \text { PH NUEVO } \\ 7.102 & .496 \mathrm{E}+00 & .480 \mathrm{E}+03 & .839 \mathrm{E}+00 & .66 \\ 8.602 & .438 \mathrm{E}+00 & .582 \mathrm{E}+03 & .886 \mathrm{E}+00 & .66 \\ 10.102 & .377 \mathrm{E}+00 & .683 \mathrm{E}+03 & .923 \mathrm{E}+00 & .66 \\ 11.602 & .312 \mathrm{E}+00 & .785 \mathrm{E}+03 & .952 \mathrm{E}+00 & .66 \\ 13.102 & .237 \mathrm{E}+00 & .886 \mathrm{E}+03 & .975 \mathrm{E}+00 & .66 \\ 14.602 & .136 \mathrm{E}+00 & .988 \mathrm{E}+03 & .991 \mathrm{E}+00 & .66\end{array}$

$\begin{array}{lllll}\mathrm{Fe}^{3+} & .800 \mathrm{E}-08 & .391 \mathrm{E}-07 & .393 \mathrm{E}-07 & .372 \mathrm{E}-07 \\ \mathrm{SO}_{4}{ }^{2-} & .727 \mathrm{E}+01 & .708 \mathrm{E}+01 & .706 \mathrm{E}+01 & .708 \mathrm{E}+01 \\ \mathrm{H}^{+} & .520 \mathrm{E}+02 & .535 \mathrm{E}+02 & .537 \mathrm{E}+02 & .535 \mathrm{E}+02 \\ \mathrm{Tu}^{2+} & .123 \mathrm{E}+02 & .126 \mathrm{E}+02 & .127 \mathrm{E}+02 & .126 \mathrm{E}+02 \\ \mathrm{Fe}^{2+} & .127 \mathrm{E}+00 & .130 \mathrm{E}+00 & .130 \mathrm{E}+00 & .130 \mathrm{E}+00 \\ \mathrm{AgTu}_{2}{ }^{+} & .105 \mathrm{E}-01 & .355 \mathrm{E}-02 & .110 \mathrm{E}-02 & .263 \mathrm{E}-02\end{array}$

$\begin{array}{llcrrrrrr} & \text { AGt } & \text { AGTt } & \text { AGT2+ } & \text { AGT3+ } & \text { AGT4+ } & \text { AG2T3+2 } & \text { AG2T6+2 } \\ \text { EN IA SUPER } & .1 \mathrm{E}-12 & .6 \mathrm{E}-06 & .6 \mathrm{E}-04 & .7 \mathrm{E}-03 & .6 \mathrm{E}-04 & .5 \mathrm{E}-02 & .97 \mathrm{E}-04 \\ \text { EN SOLUCION } & .4 \mathrm{E}-13 & .1 \mathrm{E}-06 & .2 \mathrm{E}-04 & .2 \mathrm{E}-03 & .2 \mathrm{E}-04 & .3 \mathrm{E}-03 & .66 \mathrm{E}-05\end{array}$

DIFUSIVIDAD EFECTIVA DE FE $+3=1.360907600524 \mathrm{E}-9$

$\mathrm{CONV}=.21 \mathrm{E}-01 * t+-.22 \mathrm{E}-03 * t * * 2+.46 \mathrm{E}-06 * t * * 3$ 


\section{Apéndice D - Experimento 6}

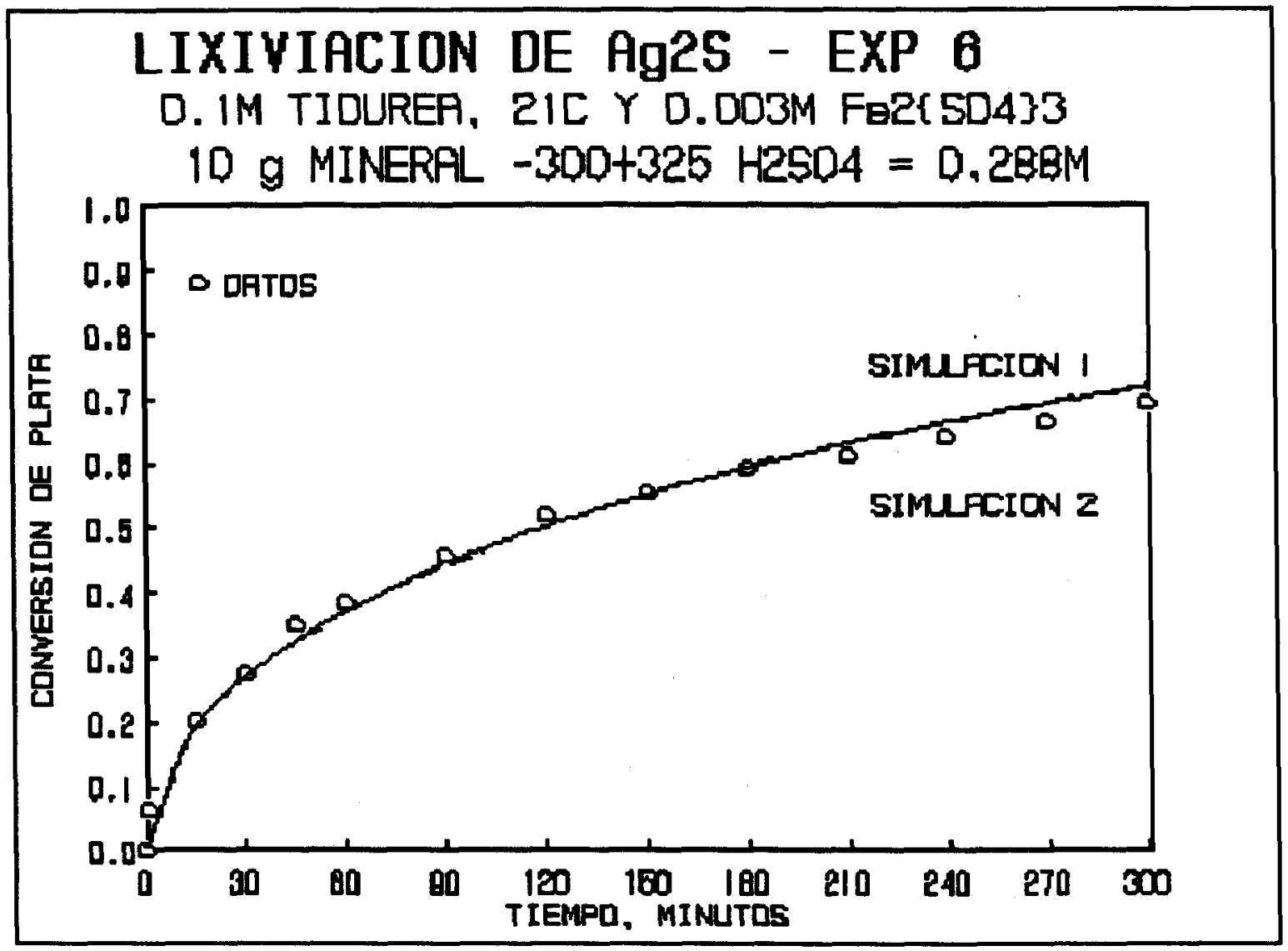




\section{Apéndice D - Experimento 7}

\section{EXPERIMENTO 7}

CONDICIONES :

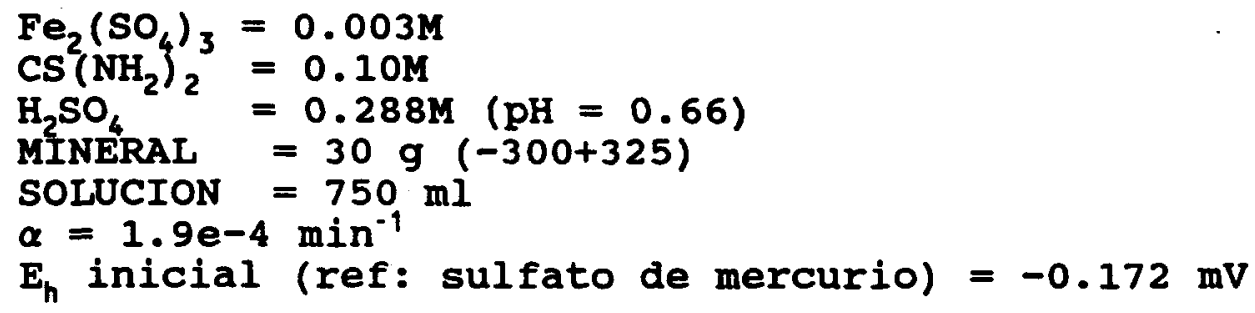

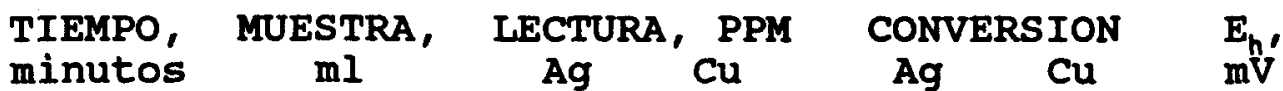

$\begin{array}{rrrrrrr}1.5 & 12 . & 16.0 & 5.1 & .0536 & .039 & -.247 \\ 15.0 & 6 . & 53.0 & 11.0 & .1756 & .084 & -.252 \\ 30.0 & 6 . & 72.0 & 13.8 & .2377 & .105 & -.254 \\ 45.0 & 6 . & 87.3 & 16.2 & .2873 & .123 & -.255 \\ 60.0 & 6 . & 101.2 & 18.7 & .3321 & .141 & -.256 \\ 90.0 & 6 . & 113.3 & 19.8 & .3706 & .149 & -.257 \\ 120.0 & 6 . & 134.8 & 20.1 & .4386 & .151 & -.259 \\ 145.0 & 6 . & 142.0 & 20.4 & .4612 & .153 & -.260 \\ 150.0 & 6 . & 145.2 & 20.4 & .4712 & .153 & -.260 \\ 180.0 & 6 . & 158.4 & 20.4 & .5119 & .153 & -.261 \\ 210.0 & 6 . & 161.7 & 21.4 & .5219 & .160 & -.262 \\ 240.0 & 6 . & 171.6 & 22.2 & .5519 & .166 & -.262 \\ 270.0 & 6 . & 174.9 & 22.2 & .5618 & .166 & -.263 \\ 295.0 & 6 . & 183.0 & 22.8 & .5859 & .170 & -.263 \\ 300.0 & 6 . & 184.8 & 22.8 & .5912 & .170 & -.263\end{array}$

RESIDUO DE Ag $=3.05 \mathrm{~kg} /$ ton

RESIDUO DE Cu $=2.70 \mathrm{~kg} /$ ton

BALANCE METALURGICO DE Ag $=7.46 \mathrm{~kg} /$ ton

BALANCE METALURGICO DE Cu $=3.25 \mathrm{~kg} /$ ton 


\title{
Apéndice D - Experimento 7
}

\author{
LIXIVIACION DE ARGENTITA \\ CARACTERISTICAS DEL MINERAL \\ GR POR TON DE AG $=.746 \mathrm{E}+04$ \\ DENSIDAD DEL MINERAL $=5.20$ \\ RADIO DE LA PART, $C M=.235 E-02$ \\ POROSIDAD $=0.000 \mathrm{E}+00$ \\ PROPORCION DE SOLIDO A LIQUIDO, KG/LT $=.400 E-01$
}

CONSTANTES DE EQUILIBRIO A 21.00C

CONCENTRACION INICIAL DE FE2(SO4) $3=.310 E-02$

CONCENTRACION INICIAL DE H2O2 $=0.000 \mathrm{E}+00$

CONCENTRACION INICIAL DE TIOUREA $=.100 \mathrm{E}+00$

GAMA $=\quad .173 E-01$

VELOCIDAD DE DESCOMPOSICION DE TIOUREA = TIME* .390E-04

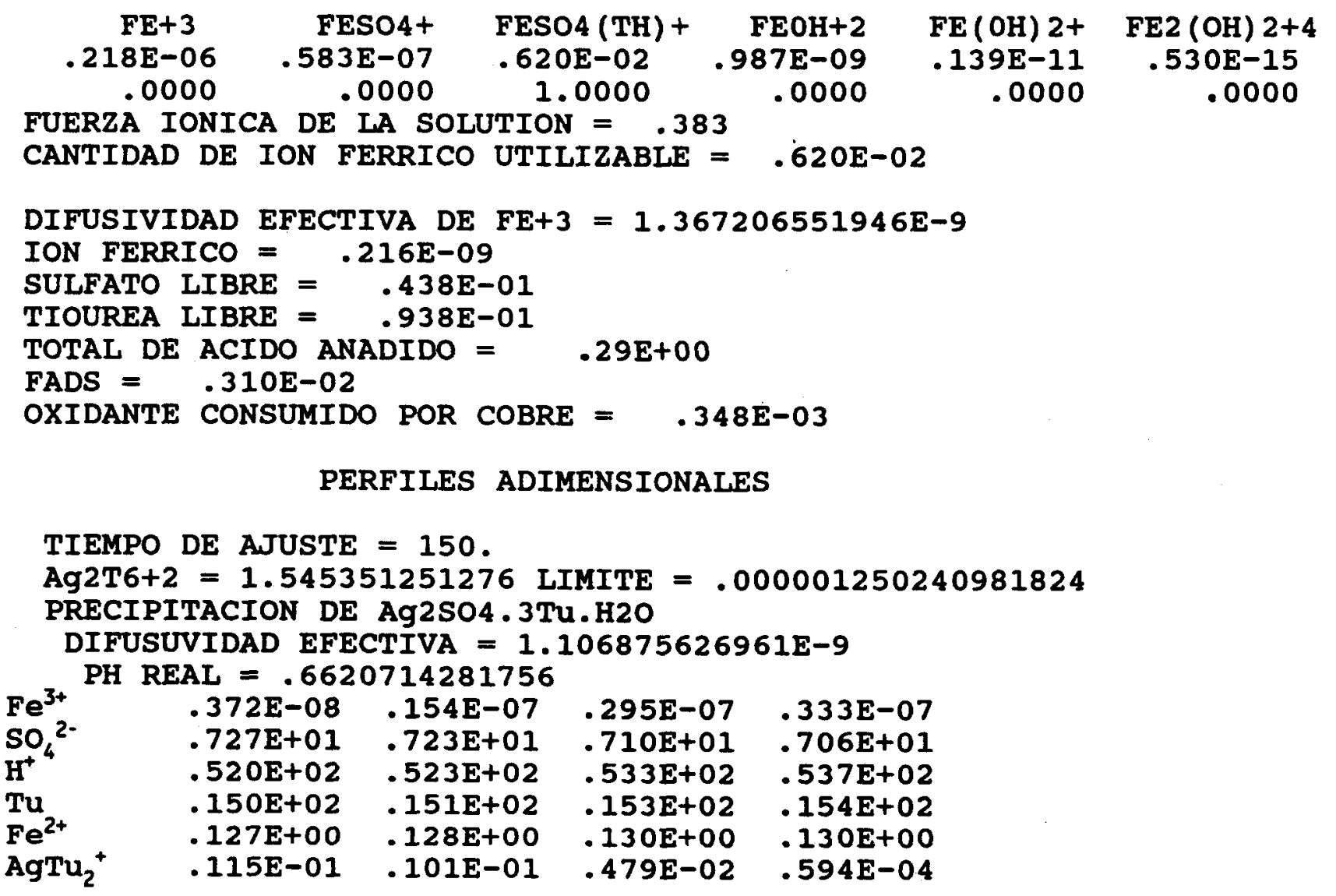




\section{Apéndice D - Experimento 7}

$\begin{array}{llcrrrrrr} & & \text { AGt } & \text { AGT+ } & \text { AGT2+ } & \text { AGT3+ } & \text { AGT4+ } & \text { AG2T3+2 } & \text { AG2T6+2 } \\ \text { EN LA SUPER } & .1 \mathrm{E}-12 & .5 \mathrm{E}-06 & .7 \mathrm{E}-04 & .9 \mathrm{E}-03 & .1 \mathrm{E}-03 & .5 \mathrm{E}-02 & .18 \mathrm{E}-03 \\ \text { EN SOLUCION } & .5 \mathrm{E}-15 & .3 \mathrm{E}-08 & .4 \mathrm{E}-0.6 & .5 \mathrm{E}-05 & .5 \mathrm{E}-06 & .1 \mathrm{E}-06 & .50 \mathrm{E}-08 \\ & \text { FESO4Tu }= & .66 \mathrm{E}-06 & & & & & & \end{array}$

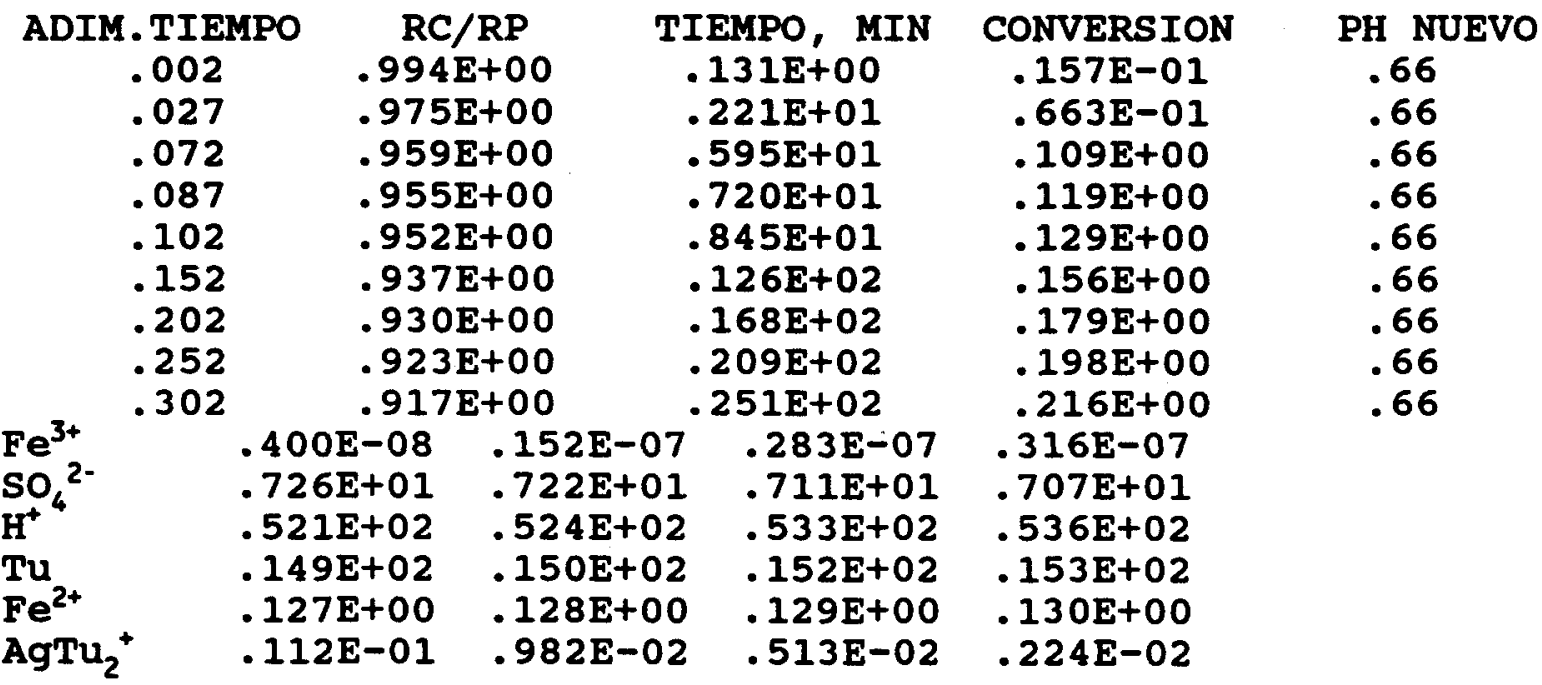

$\begin{array}{llcrrrrrr} & \text { AG+ } & \text { AGT+ } & \text { AGT2+ } & \text { AGT3+ } & \text { AGT4+ } & \text { AG2T3+2 } & \text { AG2T6+2 } \\ \text { EN LA SUPER } & .1 \mathrm{E}-12 & .5 \mathrm{E}-06 & .7 \mathrm{E}-04 & .9 \mathrm{E}-03 & .1 \mathrm{E}-03 & .5 \mathrm{E}-02 & .16 \mathrm{E}-03 \\ \text { EN SOLUCION } & .2 \mathrm{E}-13 & .1 \mathrm{E}-06 & .1 \mathrm{E}-04 & .2 \mathrm{E}-03 & .2 \mathrm{E}-04 & .2 \mathrm{E}-03 & .69 \mathrm{E}-05\end{array}$

$\begin{array}{ccccc}\text { ADIM.TIEMPO } & \text { RC/RP } & \text { TIEMPO, MIN } & \text { CONVERSION } & \text { PH NUEVO } \\ .452 & .896 \mathrm{E}+00 & .376 \mathrm{E}+02 & .260 \mathrm{E}+00 & .66 \\ .552 & .886 \mathrm{E}+00 & .459 \mathrm{E}+02 & .284 \mathrm{E}+00 & .66 \\ .652 & .878 \mathrm{E}+00 & .542 \mathrm{E}+02 & .306 \mathrm{E}+00 & .66 \\ .752 & .870 \mathrm{E}+00 & .625 \mathrm{E}+02 & .326 \mathrm{E}+00 & .66 \\ .852 & .862 \mathrm{E}+00 & .708 \mathrm{E}+02 & .345 \mathrm{E}+00 & .66 \\ 1.002 & .849 \mathrm{E}+00 & .833 \mathrm{E}+02 & .369 \mathrm{E}+00 & .66 \\ 1.152 & .839 \mathrm{E}+00 & .958 \mathrm{E}+02 & .392 \mathrm{E}+00 & .66 \\ 1.302 & .830 \mathrm{E}+00 & .108 \mathrm{E}+03 & .413 \mathrm{E}+00 & .66 \\ 1.452 & .821 \mathrm{E}+00 & .121 \mathrm{E}+03 & .432 \mathrm{E}+00 & .66 \\ 1.602 & .813 \mathrm{E}+00 & .133 \mathrm{E}+03 & .450 \mathrm{E}+00 & .66 \\ 1.902 & .791 \mathrm{E}+00 & .158 \mathrm{E}+03 & .481 \mathrm{E}+00 & .66 \\ 2.202 & .777 \mathrm{E}+00 & .183 \mathrm{E}+03 & .510 \mathrm{E}+00 & .66 \\ 2.502 & .763 \mathrm{E}+00 & .208 \mathrm{E}+03 & .535 \mathrm{E}+00 & .66 \\ 2.802 & .751 \mathrm{E}+00 & .233 \mathrm{E}+03 & .559 \mathrm{E}+00 & .66 \\ 3.102 & .739 \mathrm{E}+00 & .258 \mathrm{E}+03 & .580 \mathrm{E}+00 & .66 \\ 3.602 & .714 \mathrm{E}+00 & .299 \mathrm{E}+03 & .612 \mathrm{E}+00 & .66 \\ 4.102 & .696 \mathrm{E}+00 & .341 \mathrm{E}+03 & .641 \mathrm{E}+00 & .66 \\ 4.602 & .679 \mathrm{E}+00 & .383 \mathrm{E}+03 & .667 \mathrm{E}+00 & .66 \\ 5.102 & .663 \mathrm{E}+00 & .424 \mathrm{E}+03 & .690 \mathrm{E}+00 & .66\end{array}$




\section{Apéndice D - Experimento 7}

$\begin{array}{lllll}\mathrm{Fe}^{3+} & .511 \mathrm{E}-08 & .155 \mathrm{E}-07 & .258 \mathrm{E}-07 & .279 \mathrm{E}-07 \\ \mathrm{SO}_{4}{ }^{2-} & .723 \mathrm{E}+01 & .719 \mathrm{E}+01 & .712 \mathrm{E}+01 & .710 \mathrm{E}+01 \\ \mathrm{H}^{+} & .523 \mathrm{E}+02 & .526 \mathrm{E}+02 & .532 \mathrm{E}+02 & .534 \mathrm{E}+02 \\ \mathrm{Tu}^{2+} & .146 \mathrm{E}+02 & .146 \mathrm{E}+02 & .148 \mathrm{E}+02 & .148 \mathrm{E}+02 \\ \mathrm{Fe}^{2+} & .128 \mathrm{E}+00 & .128 \mathrm{E}+00 & .129 \mathrm{E}+00 & .130 \mathrm{E}+00 \\ \mathrm{AgTu}_{2}{ }^{+} & .103 \mathrm{E}-01 & .891 \mathrm{E}-02 & .552 \mathrm{E}-02 & .426 \mathrm{E}-02\end{array}$

$\begin{array}{llcrrrrrr} & & \text { AGt } & \text { AGT+ } & \text { AGT2+ } & \text { AGT3+ } & \text { AGT4+ } & \text { AG2T3+2 } & \text { AG2T6+2 } \\ \text { EN LA SUPER } & .1 \mathrm{E}-12 & .5 \mathrm{E}-06 & .6 \mathrm{E}-04 & .8 \mathrm{E}-03 & .8 \mathrm{E}-04 & .4 \mathrm{E}-02 & .13 \mathrm{E}-03 \\ \text { EN SOLUCION } & .4 \mathrm{E}-13 & .2 \mathrm{E}-06 & .3 \mathrm{E}-04 & .3 \mathrm{E}-03 & .4 \mathrm{E}-04 & .8 \mathrm{E}-03 & .24 \mathrm{E}-04\end{array}$

$\begin{array}{ccccc}\text { ADIM.TIEMPO } & \text { RC/RP } & \text { TIEMPO, MIN } & \text { CONVERSION } & \text { PH NUEVO } \\ 7.102 & .582 \mathrm{E}+00 & .591 \mathrm{E}+03 & .765 \mathrm{E}+00 & .66 \\ 8.602 & .544 \mathrm{E}+00 & .715 \mathrm{E}+03 & .808 \mathrm{E}+00 & .66 \\ 10.102 & .507 \mathrm{E}+00 & .840 \mathrm{E}+03 & .844 \mathrm{E}+00 & .66 \\ 11.602 & .471 \mathrm{E}+00 & .965 \mathrm{E}+03 & .875 \mathrm{E}+00 & .66 \\ 13.102 & .435 \mathrm{E}+00 & .109 \mathrm{E}+04 & .900 \mathrm{E}+00 & .67 \\ 14.602 & .399 \mathrm{E}+00 & .121 \mathrm{E}+04 & .922 \mathrm{E}+00 & .67 \\ 16.102 & .362 \mathrm{E}+00 & .134 \mathrm{E}+04 & .940 \mathrm{E}+00 & .67 \\ 17.602 & .323 \mathrm{E}+00 & .146 \mathrm{E}+04 & .956 \mathrm{E}+00 & .67 \\ 19.102 & .283 \mathrm{E}+00 & .159 \mathrm{E}+04 & .969 \mathrm{E}+00 & .67 \\ 20.602 & .239 \mathrm{E}+00 & .171 \mathrm{E}+04 & .980 \mathrm{E}+00 & .67\end{array}$

$\begin{array}{lllll}\mathrm{Fe}^{3+} & .763 \mathrm{E}-08 & .287 \mathrm{E}-07 & .286 \mathrm{E}-07 & .268 \mathrm{E}-07 \\ \mathrm{SO}_{4}{ }^{2-} & .721 \mathrm{E}+01 & .709 \mathrm{E}+01 & .709 \mathrm{E}+01 & .711 \mathrm{E}+01 \\ \mathrm{H}^{+} & .524 \mathrm{E}+02 & .534 \mathrm{E}+02 & .534 \mathrm{E}+02 & .533 \mathrm{E}+02 \\ \mathrm{Tu} & .136 \mathrm{E}+02 & .138 \mathrm{E}+02 & .138 \mathrm{E}+02 & .137 \mathrm{E}+02 \\ \mathrm{Fe}^{2+} & .128 \mathrm{E}+00 & .130 \mathrm{E}+00 & .130 \mathrm{E}+00 & .129 \mathrm{E}+00 \\ \mathrm{AgTu}_{2}{ }^{+} & .937 \mathrm{E}-02 & .403 \mathrm{E}-02 & .405 \mathrm{E}-02 & .501 \mathrm{E}-02\end{array}$

$\begin{array}{llcrrrrrr} & & \text { AGt } & \text { AGT+ } & \text { AGT2+ } & \text { AGT3+ } & \text { AGT4+ } & \text { AG2T3+2 } & \text { AG2T6+2 } \\ \text { EN LA SUPER } & .1 \mathrm{E}-12 & .5 \mathrm{E}-06 & .6 \mathrm{E}-04 & .7 \mathrm{E}-03 & .7 \mathrm{TE}-04 & .4 \mathrm{E}-02 & .10 \mathrm{E}-03 \\ \text { EN SOLUCION } & .6 \mathrm{E}-13 & .2 \mathrm{E}-06 & .3 \mathrm{E}-04 & .4 \mathrm{E}-03 & .4 \mathrm{E}-04 & .1 \mathrm{E}-02 & .29 \mathrm{E}-04 \\ & \text { FeSO4TU }= & .12 \mathrm{E}-05 & & & & & & \end{array}$

DIFUSIVIDAD EFECTIVA DE FE+3 $=1.106875626961 \mathrm{E}-9$

CONV $=.16 \mathrm{E}-01 * t+-.12 \mathrm{E}-03 * t * * 2+.16 \mathrm{E}-06 * t * * 3$ 
Apéndice D - Experimento 7

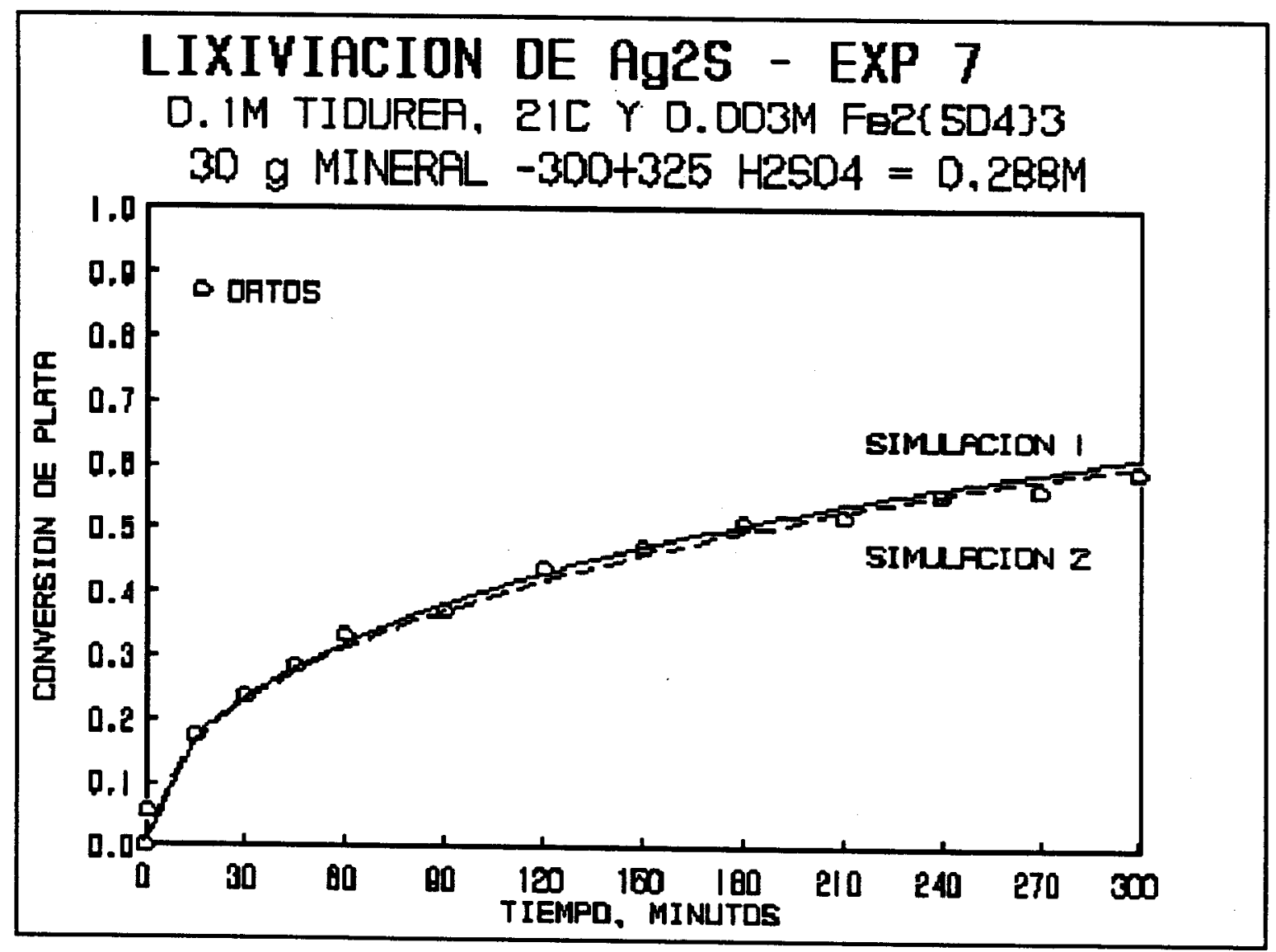




\section{EXPERIMENTO 8}

\section{CONDICIONES :}

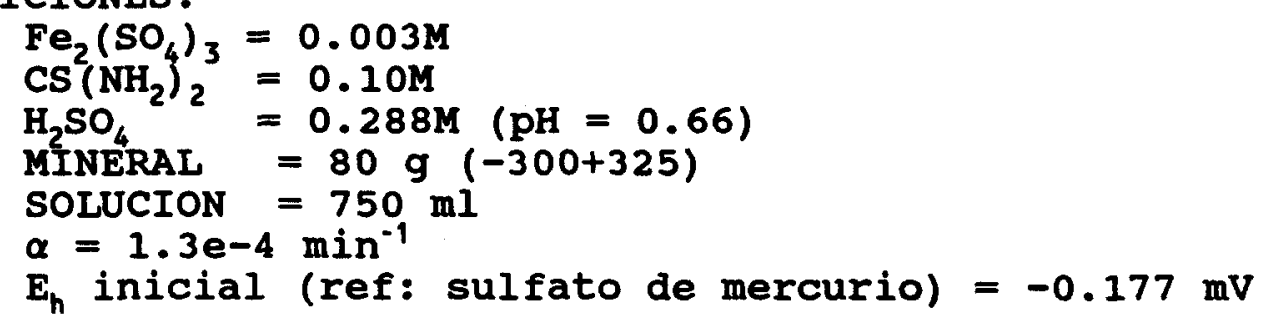

\begin{tabular}{|c|c|c|c|}
\hline $\begin{array}{l}\text { TIEMI } \\
\text { minut }\end{array}$ & $\begin{array}{c}\text { MUESTRA } \\
\mathrm{m} 1\end{array}$ & $\begin{array}{c}\text { LECTURA } \\
\text { Aq }\end{array}$ & PP \\
\hline
\end{tabular}

$\begin{array}{rrrrrrr}1.8 & 12 . & 48.3 & 14.7 & .0628 & .041 & -.251 \\ 15.0 & 6 . & 115.5 & 27.3 & .1489 & .075 & -.254 \\ 30.0 & 6 . & 147.0 & 33.6 & .1889 & .092 & -.256 \\ 45.0 & 6 . & 172.2 & 37.8 & .2206 & .103 & -.258 \\ 61.0 & 6 . & 205.0 & 45.0 & .2616 & .122 & -.259 \\ 91.0 & 6 . & 230.0 & 45.0 & .2926 & .122 & -.261 \\ 122.0 & 6 . & 265.0 & 50.0 & .3356 & .135 & -.262 \\ 148.0 & 6 . & 274.0 & 50.0 & .3465 & .135 & -.264 \\ 150.0 & 6 . & 275.0 & 50.0 & .3477 & .135 & -.264 \\ 180.0 & 6 . & 310.0 & 55.0 & .3896 & .148 & -.265 \\ 210.0 & 6 . & 315.0 & 55.0 & .3956 & .148 & -.267 \\ 240.0 & 6 . & 330.0 & 55.0 & .4132 & .148 & -.268 \\ 270.0 & 6 . & 335.0 & 55.0 & .4190 & .148 & -.269 \\ 295.0 & 6 . & 348.0 & 55.0 & .4340 & .148 & -.270 \\ 300.0 & 6 . & 355.0 & 55.0 & .4421 & .148 & -.270\end{array}$

RESIDUO DE Ag $=4.02 \mathrm{~kg} / \mathrm{ton}$

RESIDUO DE $\mathrm{Cu}=2.90 \mathrm{~kg} /$ ton

BALANCE METALURGICO DE Ag $=7.21 \mathrm{~kg} / \mathrm{ton}$

BALANCE METALURGICO DE $\mathrm{Cu}=3.40 \mathrm{~kg} /$ ton 
Apéndice D - Experimento 8

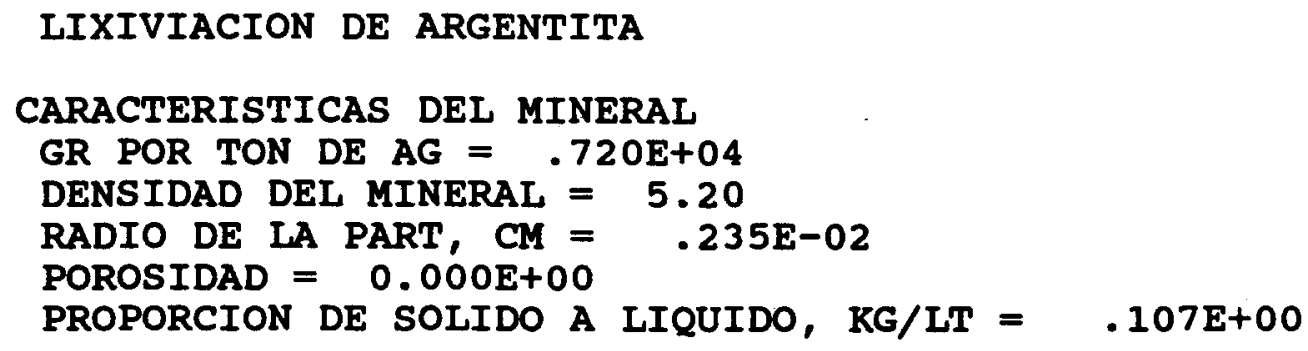

PERFILES ADIMENSIONALES

TIEMPO DE AJUSTE $=45$.

Ag2T6+2 $=.3030764286422$ LIMITE $=.000002823132451201$

PRECIPITACION DE Ag2SO4 . 3Tu.H2O

DIFUSUVIDAD EFECTIVA $=8.144504198497 \mathrm{E}-10$

PH REAL $=.6620714281756$

$\begin{array}{lllll}\mathrm{Fe}^{3+} & .373 \mathrm{E}-08 & .144 \mathrm{E}-07 & .275 \mathrm{E}-07 & .310 \mathrm{E}-07 \\ \mathrm{SO}^{2-} & .725 \mathrm{E}+01 & .721 \mathrm{E}+01 & .710 \mathrm{E}+01 & .706 \mathrm{E}+01 \\ \mathrm{H}^{+} & .521 \mathrm{E}+02 & .525 \mathrm{E}+02 & .534 \mathrm{E}+02 & .537 \mathrm{E}+02 \\ \mathrm{Tu}^{2+} & .154 \mathrm{E}+02 & .155 \mathrm{E}+02 & .157 \mathrm{E}+02 & .158 \mathrm{E}+02 \\ \mathrm{Fe}^{2+} & .127 \mathrm{E}+00 & .128 \mathrm{E}+00 & .130 \mathrm{E}+00 & .130 \mathrm{E}+00 \\ \mathrm{AgTu}_{2}{ }^{+} & .110 \mathrm{E}-01 & .970 \mathrm{E}-02 & .456 \mathrm{E}-02 & .137 \mathrm{E}-03\end{array}$




\section{Apéndice D - Experimento 8}

$\begin{array}{llcrrrrrr} & & \text { AGt } & \text { AGT+ } & \text { AGT2+ } & \text { AGT3+ } & \text { AGT4+ } & \text { AG2T3+2 } & \text { AG2T6+2 } \\ \text { EN LA SUPER } & .1 \mathrm{E}-12 & .5 \mathrm{E}-06 & .7 \mathrm{E}-04 & .9 \mathrm{E}-03 & .1 \mathrm{E}-03 & .5 \mathrm{E}-02 & .17 \mathrm{E}-03 \\ \text { EN SOLUCION } & .1 \mathrm{E}-14 & .6 \mathrm{E}-08 & .8 \mathrm{E}-0.6 & .1 \mathrm{E}-04 & .1 \mathrm{E}-05 & .7 \mathrm{E}-06 & .28 \mathrm{E}-07 \\ & \text { FeSO4Tu }= & .68 \mathrm{E}-06 & .66 & & & & & \end{array}$

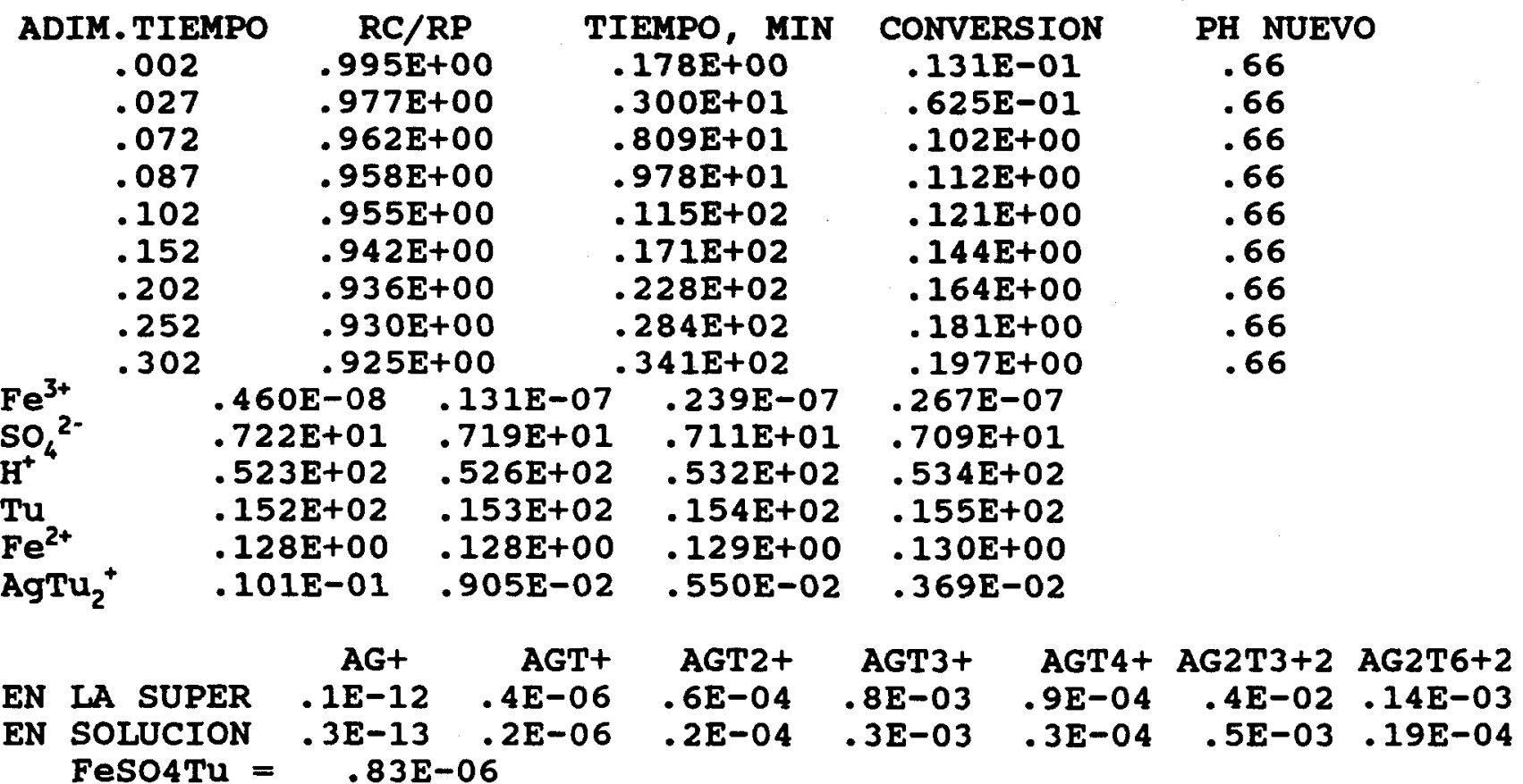

$\begin{array}{cc}\text { ADIM.TIEMPO } & \text { RC/RP } \\ .452 & .908 \mathrm{E}+00 \\ .552 & .901 \mathrm{E}+00 \\ .652 & .894 \mathrm{E}+00 \\ .752 & .888 \mathrm{E}+00 \\ .852 & .882 \mathrm{E}+00 \\ 1.002 & .873 \mathrm{E}+00 \\ 1.152 & .866 \mathrm{E}+00 \\ 1.302 & .860 \mathrm{E}+00 \\ 1.452 & .854 \mathrm{E}+00 \\ 1.602 & .848 \mathrm{E}+00 \\ 1.902 & .835 \mathrm{E}+00 \\ 2.202 & .826 \mathrm{E}+00 \\ 2.502 & .819 \mathrm{E}+00 \\ 2.802 & .812 \mathrm{E}+00 \\ 3.102 & .806 \mathrm{E}+00 \\ 3.602 & .794 \mathrm{E}+00 \\ 4.102 & .786 \mathrm{E}+00 \\ 4.602 & .780 \mathrm{E}+00 \\ 5.102 & .774 \mathrm{E}+00\end{array}$
TIEMPO, MIN
$.510 \mathrm{E}+02$
$.623 \mathrm{E}+02$
$.736 \mathrm{E}+02$
$.849 E+02$
$.962 \mathrm{E}+02$
$.113 E+03$
$.130 \mathrm{E}+03$
$.147 \mathrm{E}+03$
$.164 \mathrm{E}+03$
$.181 \mathrm{E}+03$
$.215 E+03$
$.249 \mathrm{E}+03$
$.283 \mathrm{E}+03$
$.317 \mathrm{E}+03$
$.351 \mathrm{E}+03$
$.407 \mathrm{E}+03$
$.464 \mathrm{E}+03$
$.520 \mathrm{E}+03$
$.577 \mathrm{E}+03$

\section{CONVERSION}

$.233 \mathrm{E}+00$

$.253 \mathrm{E}+00$

$.271 \mathrm{E}+00$

$.287 \mathrm{E}+00$

$.301 E+00$

$.320 \mathrm{E}+00$

$.337 \mathrm{E}+00$

$.353 E+00$

$.367 \mathrm{E}+00$

$.379 E+00$

$.401 \mathrm{E}+00$

$.421 \mathrm{E}+00$

$.438 \mathrm{E}+00$

$.453 \mathrm{E}+00$

$.466 \mathrm{E}+00$

$.485 \mathrm{E}+00$

$.501 \mathrm{E}+00$

$.515 \mathrm{E}+00$

$.527 \mathrm{E}+00$
PH NUEVO

.66

.66

.66

.66

.66

.66

.66

.67

.67

.67

.67

.67

.67

.67

.67

.67

.67

.67

.67 
Apéndice D - Experimento 8

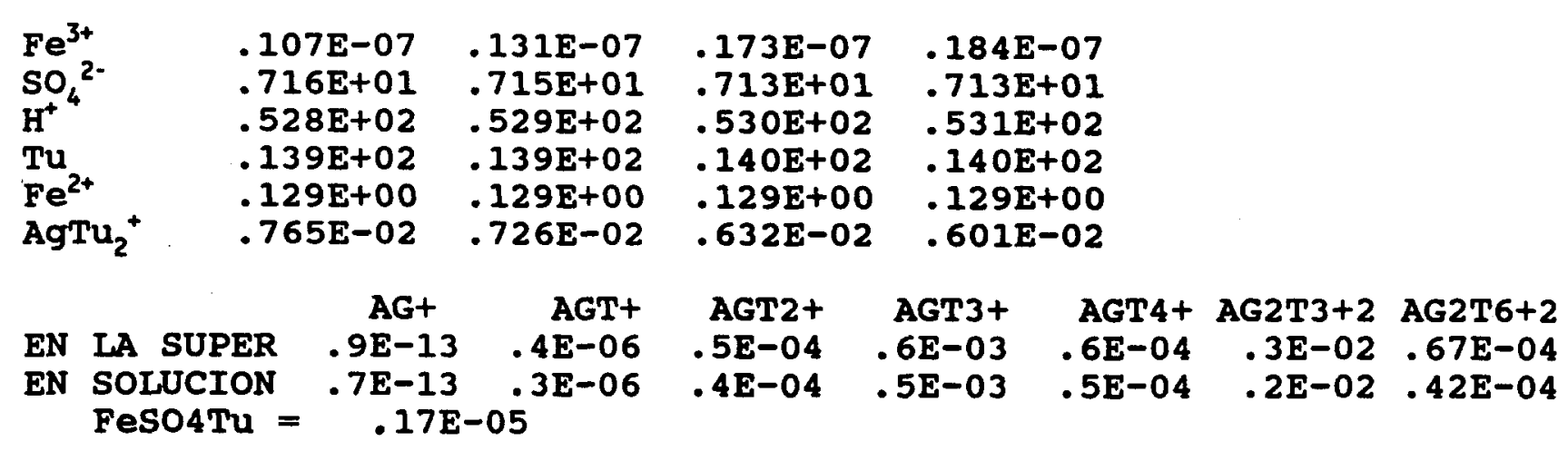




\section{Apéndice D - Experimento 8}

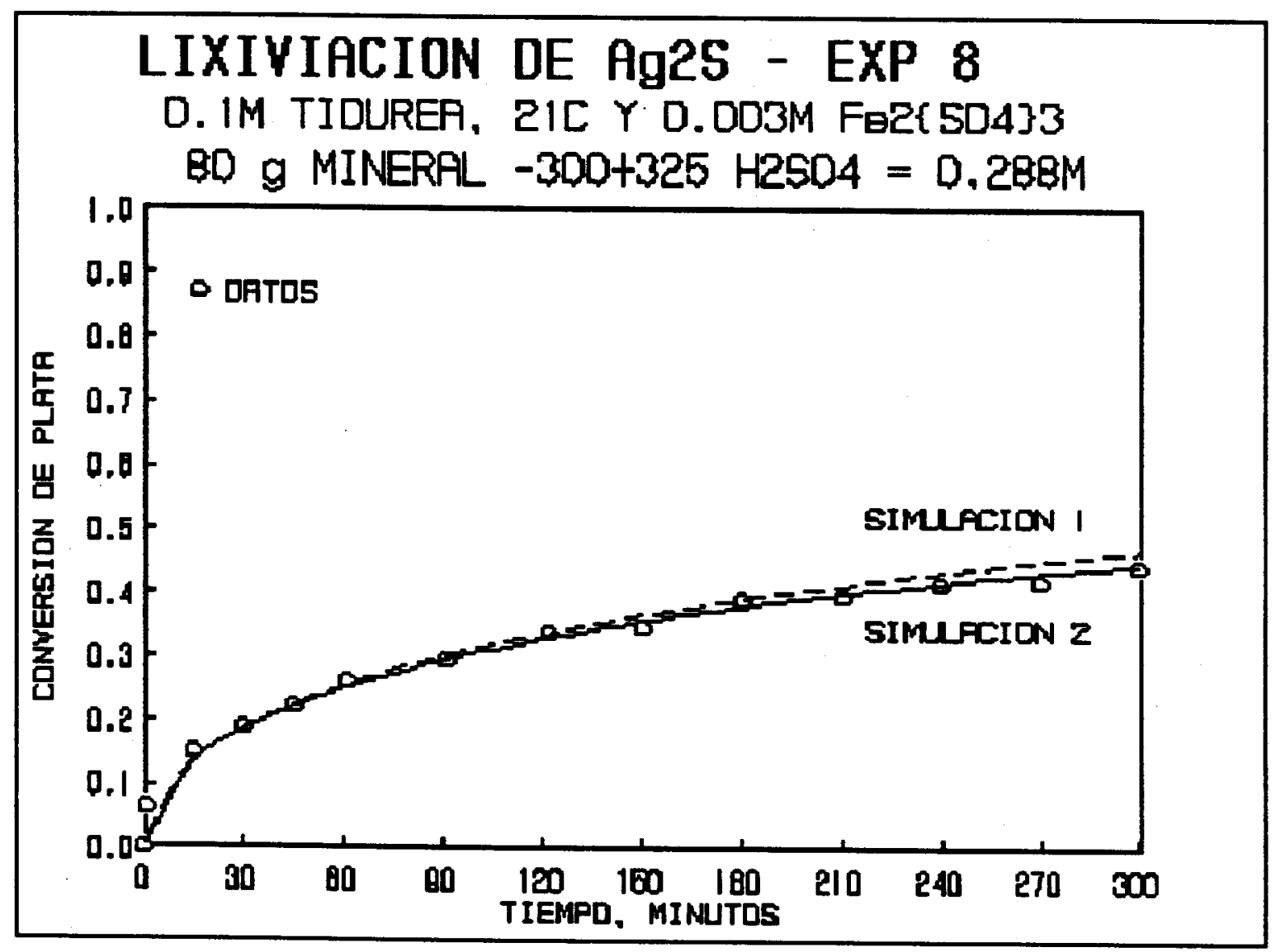




\section{Apéndice D - Experimento 9}

\section{EXPERIMENTO 9}

CONDICIONES :

$$
\begin{aligned}
& \mathrm{Fe}_{2}\left(\mathrm{SO}_{4}\right)_{3}=0.003 \mathrm{M} \\
& \mathrm{CS}\left(\mathrm{NH}_{2}\right)_{2}=0.10 \mathrm{M} \\
& \mathrm{H}_{2} \mathrm{SO}_{4}=0.040 \mathrm{M}(\mathrm{pH}=1.45) \\
& \mathrm{MINERAL}=10 \mathrm{~g}(-300+325) \\
& \text { SOLUCION }=750 \mathrm{mI} \\
& \alpha=1.3 \mathrm{e}-4 \mathrm{~min}^{-1} \\
& \mathrm{E}_{\mathrm{h}} \text { inicial (ref: sulfato de mercurio) }=-0.137 \mathrm{mV}
\end{aligned}
$$

\begin{tabular}{|c|c|c|c|c|c|c|}
\hline $\begin{array}{l}\text { TIEMPO, } \\
\text { minutos }\end{array}$ & $\begin{array}{c}\text { MUESTRA, } \\
\text { ml }\end{array}$ & $\begin{array}{l}\text { LECTURA, } \\
\text { Ag }\end{array}$ & $\begin{array}{l}\mathrm{PPM} \\
\mathrm{Cu}\end{array}$ & $\begin{array}{c}\text { CONV } \\
\text { Ag }\end{array}$ & $\begin{array}{l}\text { ION } \\
\mathrm{Cu}\end{array}$ & $\begin{array}{l}E_{h^{\prime}} \\
m^{\prime} \mathbf{v}\end{array}$ \\
\hline $\begin{array}{r}1.5 \\
15.0 \\
30.0 \\
45.0 \\
60.0 \\
90.0 \\
120.0 \\
145.0 \\
150.0 \\
180.0 \\
209.0 \\
240.0 \\
270.0 \\
295.0 \\
300.0\end{array}$ & $\begin{array}{l}12 . \\
6 . \\
6 . \\
6 . \\
6 . \\
6 . \\
6 . \\
6 . \\
6 . \\
6 . \\
6 . \\
6 . \\
6 . \\
6 . \\
6 .\end{array}$ & $\begin{array}{l}10.0 \\
21.5 \\
29.5 \\
36.0 \\
41.4 \\
49.5 \\
55.0 \\
59.6 \\
60.5 \\
61.5 \\
64.9 \\
66.0 \\
69.3 \\
73.9 \\
74.8\end{array}$ & $\begin{array}{l}2.4 \\
4.1 \\
5.1 \\
5.7 \\
6.1 \\
6.7 \\
6.9 \\
7.2 \\
7.2 \\
7.3 \\
7.3 \\
7.3 \\
7.4 \\
7.5 \\
7.5\end{array}$ & $\begin{array}{l}.0956 \\
.2039 \\
.2785 \\
.3387 \\
.3883 \\
.4620 \\
.5117 \\
.5529 \\
.5609 \\
.5697 \\
.5993 \\
.6088 \\
.6371 \\
.6762 \\
.6838\end{array}$ & $\begin{array}{l}.057 \\
.097 \\
.120 \\
.134 \\
.143 \\
.157 \\
.161 \\
.168 \\
.168 \\
.170 \\
.170 \\
.170 \\
.172 \\
.174 \\
.174\end{array}$ & $\begin{array}{l}-.190 \\
-.213 \\
-.214 \\
-.215 \\
-.216 \\
-.217 \\
-.218 \\
-.219 \\
-.219 \\
-.220 \\
-.221 \\
-.222 \\
-.222 \\
-.223 \\
-.223\end{array}$ \\
\hline
\end{tabular}

RESIDUO DE Ag $=2.48 \mathrm{~kg} /$ ton

RESIDUO DE Cu $=2.80 \mathrm{~kg} /$ ton

BALANCE METALURGICO DE Ag $=7.84 \mathrm{~kg} /$ ton

BALANCE METALURGICO DE $\mathrm{Cu}=3.15 \mathrm{~kg} / \mathrm{ton}$ 
Apéndice D - Experimento 9

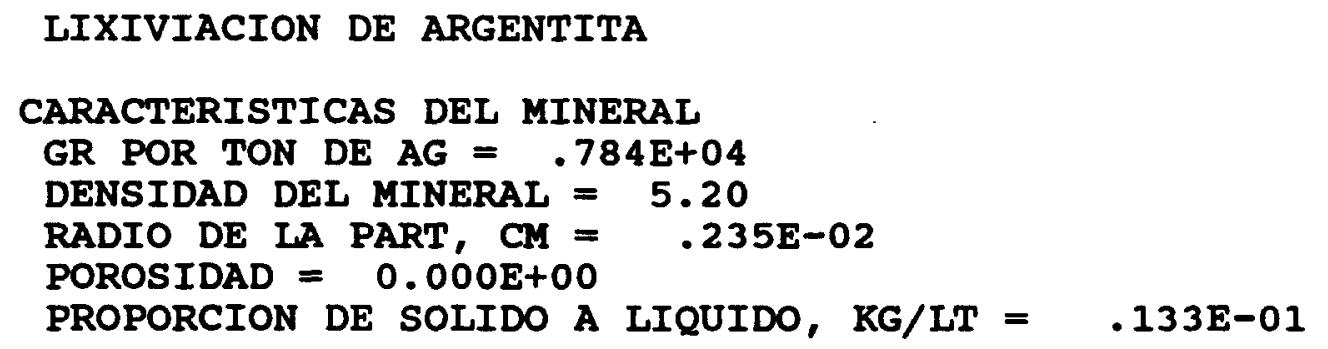

CONSTANTES DE EQUILIBRIO A $21.00 \mathrm{C}$

CONCENTRACION INICIAL DE FE2 (SO4) $3=.310 E-02$

CONCENTRACION INICIAL DE H2O2 $=0.000 \mathrm{E}+00$

CONCENTRACION INICIAL DE TIOUREA $=.100 E+00$

GAMA $=.164 \mathrm{E}-01$

VELOCIDAD DE DESCOMPOSICION DE TIOUREA = TIME* .130E-03

$\begin{array}{crrrrr}\text { FE+3 } & \text { FESO4+ } & \text { FESO4 }(\mathrm{TH})+ & \text { FEOH+2 } & \text { FE(OH) 2+ } & \text { FE2(OH) } 2+4 \\ .140 \mathrm{OE}-06 & .749 \mathrm{E}-09 & .620 \mathrm{E}-02 & .685 \mathrm{E}-08 & .457 \mathrm{E}-10 & .146 \mathrm{E}-13 \\ .0000 & .0000 & 1.0002 & .0000 & .0000 & .0000\end{array}$

FUERZA IONICA DE LA SOLUTION $=.080$

CANTIDAD DE ION FERRICO UTILIZABLE $=.620 \mathrm{E}-02$

DIFUSIVIDAD EFECTIVA DE FE+3 $=1.510040520655 \mathrm{E}-9$

ION FERRICO $=.831 \mathrm{E}-10$

SULFATO LIBRE $=.159 \mathrm{E}-01$

TIOUREA LIBRE $=.938 \mathrm{E}-01$

TOTAL DE ACIDO ANADIDO $=.40 \mathrm{E}-01$

FADS $=.310 \mathrm{E}-02$

OXIDANTE CONSUMIDO POR COBRE $=.115 \mathrm{E}-03$

\section{PERFILES ADIMENSIONALES}

TIEMPO DE AJUSTE $=180$.

Ag2T6+2 $=.0001790390829868$ LIMITE $=.00004055301356025$

PRECIPITACION DE Ag2SO4.3Tu.H2O

DIFUSUVIDAD EFECTIVA $=1.372636606649 \mathrm{E}-9$

3+ PH REAL $=1.450476826062$

$\begin{array}{lllll}\mathrm{Fe}^{3+} & .233 \mathrm{E}-08 & .553 \mathrm{E}-08 & .113 \mathrm{E}-07 & .132 \mathrm{E}-07 \\ \mathrm{SO}_{4}{ }^{2-} & .291 \mathrm{E}+01 & .283 \mathrm{E}+01 & .262 \mathrm{E}+01 & .256 \mathrm{E}+01 \\ \mathrm{H}^{+} & .774 \mathrm{E}+01 & .804 \mathrm{E}+01 & .889 \mathrm{E}+01 & .917 \mathrm{E}+01 \\ \mathrm{Tu} & .148 \mathrm{E}+02 & .149 \mathrm{E}+02 & .151 \mathrm{E}+02 & .152 \mathrm{E}+02 \\ \mathrm{Fe}^{2+} & .267 \mathrm{E}+00 & .273 \mathrm{E}+00 & .287 \mathrm{E}+00 & .292 \mathrm{E}+00 \\ \mathrm{AgTu}_{2}{ }^{+} & .115 \mathrm{E}-01 & .101 \mathrm{E}-01 & .478 \mathrm{E}-02 & .212 \mathrm{E}-04\end{array}$




\section{Apéndice D - Experimento 9}

$\begin{array}{llcrrrrrr} & & \text { AGt } & \text { AGT+ } & \text { AGT2+ } & \text { AGT3+ } & \text { AGT4+ } & \text { AG2T3+2 } & \text { AG2T6+2 } \\ \text { EN LA SUPER } & .1 \mathrm{E}-12 & .5 \mathrm{E}-06 & .7 \mathrm{E}-04 & .9 \mathrm{E}-03 & .1 \mathrm{E}-03 & .6 \mathrm{E}-02 & .17 \mathrm{E}-03 \\ \text { EN SOLUCION } & .2 \mathrm{E}-15 & .9 \mathrm{E}-09 & .1 \mathrm{E}-06 & .2 \mathrm{E}-05 & .2 \mathrm{E}-06 & .2 \mathrm{E}-07 & .61 \mathrm{E}-09 \\ & \text { FeSO4Tu }= & .68 \mathrm{E}-06 & & & & & & \end{array}$

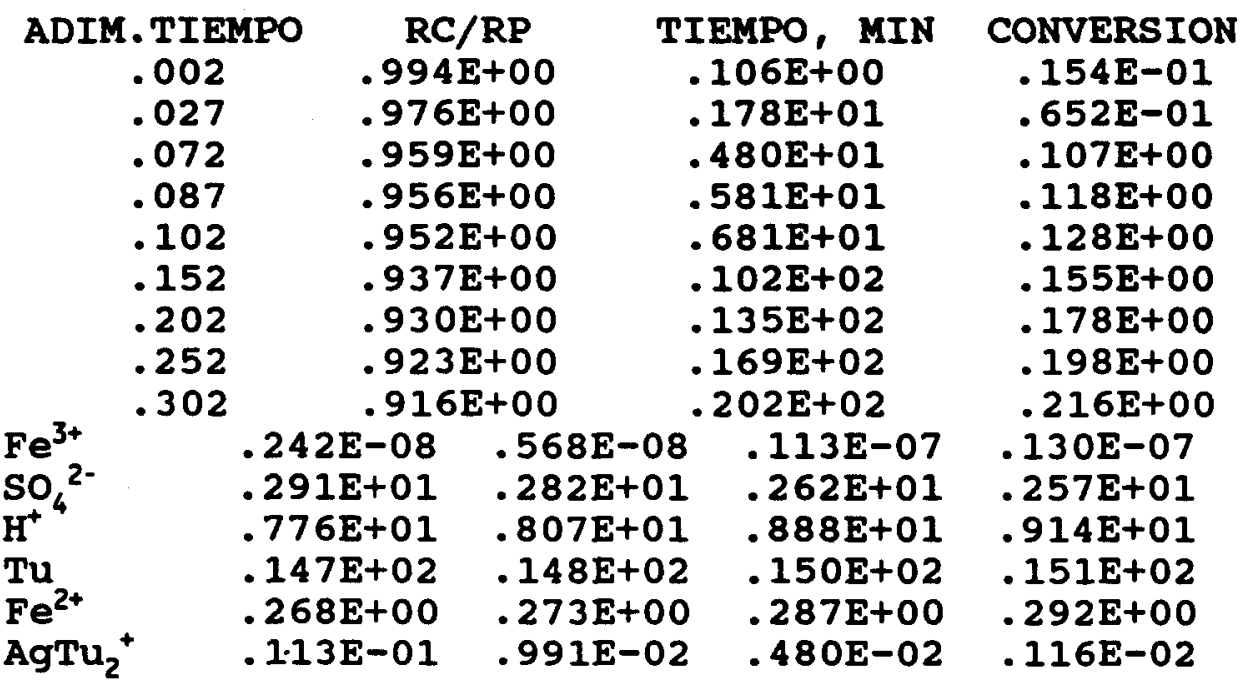

$\begin{array}{llcrrrrrr} & & \text { AGt } & \text { AGT+ } & \text { AGT2+ } & \text { AGT3+ } & \text { AGT4+ } & \text { AG2T3+2 } & \text { AG2T6+2 } \\ \text { EN IA SUPER } & .1 \mathrm{E}-12 & .5 \mathrm{E}-06 & .7 \mathrm{E}-04 & .9 \mathrm{E}-03 & .1 \mathrm{E}-03 & .5 \mathrm{E}-02 & .16 \mathrm{E}-03 \\ \text { EN SOLUCION } & .1 \mathrm{E}-13 & .5 \mathrm{E}-07 & .7 \mathrm{E}-05 & .9 \mathrm{E}-04 & .1 \mathrm{E}-04 & .5 \mathrm{E}-04 & .18 \mathrm{E}-05\end{array}$

ADIM.TIEMPO
.452
.552
.652
.752
.852
1.002
1.152
1.302
1.452
1.602
1.902
2.202
2.502
2.802
3.102
3.602
4.102
4.602
5.102

RC/RP

$.895 \mathrm{E}+00$

$.885 \mathrm{E}+00$

$.876 \mathrm{E}+00$

$.868 \mathrm{E}+00$

$.859 \mathrm{E}+00$

$.845 \mathrm{E}+00$

$.835 \mathrm{E}+00$

$.825 \mathrm{E}+00$

$.815 \mathrm{E}+00$

$.806 \mathrm{E}+00$

$.782 \mathrm{E}+00$

$.766 \mathrm{E}+00$

$.751 \mathrm{E}+00$

$.737 \mathrm{E}+00$

$.723 \mathrm{E}+00$

$.694 \mathrm{E}+00$

$.674 \mathrm{E}+00$

$.654 \mathrm{E}+00$

$.635 \mathrm{E}+00$
TIEMPO, MIN
$.303 \mathrm{E}+02$
$.370 \mathrm{E}+02$
$.437 \mathrm{E}+02$
$.504 E+02$
$.571 \mathrm{E}+02$
$.672 \mathrm{E}+02$
$.772 \mathrm{E}+02$
$.873 E+02$
$.973 E+02$
$.107 \mathrm{E}+03$
$.128 \mathrm{E}+03$
$.148 \mathrm{E}+03$
$.168 \mathrm{E}+03$
$.188 \mathrm{E}+03$
$.208 \mathrm{E}+03$
$.242 E+03$
$.275 \mathrm{E}+03$
$.309 E+03$
$.342 \mathrm{E}+03$

PH NUEVO

1.45

1.45

1.45

1.45

1.45

1.45

1.45

1.45

1.45

\section{0}


Apéndice D - Experimento 9

\begin{tabular}{|c|c|c|c|c|c|c|c|c|}
\hline \multirow{2}{*}{$\begin{array}{l}\mathrm{Fe}^{3+} \\
\mathrm{SO}_{4}^{2-} \\
\mathrm{H}^{+} \\
\mathrm{Tu}^{2+} \\
\mathrm{Fe}^{2+} \\
\mathrm{AgTu}_{2} \\
\mathrm{EN} \mathrm{L}\end{array}$} & \multicolumn{2}{|c|}{$\begin{array}{l}324 \mathrm{E}-08 \\
.288 \mathrm{E}+01 \\
.784 \mathrm{E}+01 \\
.139 \mathrm{E}+02 \\
.269 \mathrm{E}+00 \\
.107 \mathrm{E}-01\end{array}$} & $\begin{array}{r}.687 \mathrm{E}-08 \\
.278 \mathrm{E}+01 \\
.822 \mathrm{E}+01 \\
.140 \mathrm{E}+02 \\
.276 \mathrm{E}+00 \\
.897 \mathrm{E}-02 \\
\\
\mathrm{AGT}+ \\
5 \mathrm{E}-06\end{array}$ & \multirow{2}{*}{$\begin{array}{l}.118 E-07 \\
.262 E+01 \\
.891 E+01 \\
.142 E+02 \\
.288 E+00 \\
.443 E-02 \\
\\
\text { AGT2+ } \\
.7 E-04\end{array}$} & \multicolumn{2}{|c|}{$\begin{array}{l}.130 \mathrm{E}-07 \\
.258 \mathrm{E}+01 \\
.908 \mathrm{E}+01 \\
.143 \mathrm{E}+02 \\
.291 \mathrm{E}+00 \\
.238 \mathrm{E}-02\end{array}$} & \multirow[b]{2}{*}{$\begin{array}{r}A G 2 T 3+2 \\
.5 E-02 \\
.2 E-03\end{array}$} & \multirow[b]{2}{*}{$\begin{array}{l}\text { AG2T6+2 } \\
.13 E-03 \\
.68 E-05\end{array}$} \\
\hline & $\begin{array}{l}\text { A SUPER } \\
\text { SOLUCION } \\
\text { TESO4TU }=\end{array}$ & $\begin{array}{r}\text { AG+ } \\
.1 E-12 \\
.3 E-13 \\
.88 E\end{array}$ & $\begin{array}{r}.5 \mathrm{EGT+} \\
.1 \mathrm{E}-06 \\
-06^{1 \mathrm{E}}\end{array}$ & & $\begin{array}{l}\text { AGT3+ } \\
.8 E-03 \\
.2 E-03\end{array}$ & $\begin{array}{r}\text { AGT4+ } \\
.8 E-04 \\
.2 E-04\end{array}$ & & \\
\hline
\end{tabular}

\begin{tabular}{|c|c|c|c|c|c|c|c|c|c|}
\hline \multicolumn{3}{|c|}{$\begin{array}{c}\text { ADIM. TIEMPO } \\
7.102 \\
8.602 \\
10.102 \\
11.602 \\
13.102 \\
14.602 \\
16.102 \\
17.602 \\
19.102\end{array}$} & \multicolumn{2}{|c|}{$\begin{array}{c}\text { RC/RP } \\
.537 \mathrm{E}+00 \\
.488 \mathrm{E}+00 \\
.440 \mathrm{E}+00 \\
.392 \mathrm{E}+00 \\
.342 \mathrm{E}+00 \\
.291 \mathrm{E}+00 \\
.236 \mathrm{E}+00 \\
.175 \mathrm{E}+00 \\
.104 \mathrm{E}+00\end{array}$} & $\begin{array}{c}\text { TIEMPO, MIN } \\
.476 \mathrm{E}+03 \\
.577 \mathrm{E}+03 \\
.677 \mathrm{E}+03 \\
.778 \mathrm{E}+03 \\
.879 \mathrm{E}+03 \\
.979 \mathrm{E}+03 \\
.108 \mathrm{E}+04 \\
.118 \mathrm{E}+04 \\
.128 \mathrm{E}+04\end{array}$ & \multicolumn{2}{|c|}{$\begin{array}{c}\text { CONVERSION } \\
.806 \mathrm{E}+00 \\
.852 \mathrm{E}+00 \\
.890 \mathrm{E}+00 \\
.920 \mathrm{E}+00 \\
.944 \mathrm{E}+00 \\
.964 \mathrm{E}+00 \\
.978 \mathrm{E}+00 \\
.989 \mathrm{E}+00 \\
.996 \mathrm{E}+00\end{array}$} & \multicolumn{2}{|c|}{$\begin{array}{l}\text { PH NUEVO } \\
1.46 \\
1.46 \\
1.46 \\
1.46 \\
1.46 \\
1.46 \\
1.46 \\
1.46 \\
1.46\end{array}$} \\
\hline $\begin{array}{l}\mathrm{SO}_{4}{ }^{2} \\
\mathrm{H}^{+} \\
\mathrm{Tu}^{2+} \\
\mathrm{Fe}^{2+}\end{array}$ & & \multicolumn{2}{|c|}{$\begin{array}{l}.111 \mathrm{E}-07 \\
.270 \mathrm{E}+01 \\
.851 \mathrm{E}+01 \\
.121 \mathrm{E}+02 \\
.281 \mathrm{E}+00 \\
.694 \mathrm{E}-02\end{array}$} & $\begin{array}{l}.160 \mathrm{E}-07 \\
.255 \mathrm{E}+01 \\
.918 \mathrm{E}+01 \\
.123 \mathrm{E}+02 \\
.293 \mathrm{E}+00 \\
.791 \mathrm{E}-03\end{array}$ & $\begin{array}{l}.156 \mathrm{E}-07 \\
.257 \mathrm{E}+01 \\
.911 \mathrm{E}+01 \\
.123 \mathrm{E}+02 \\
.292 \mathrm{E}+00 \\
.782 \mathrm{E}-03\end{array}$ & \multicolumn{2}{|c|}{$\begin{array}{l}.149 \mathrm{E}-07 \\
.258 \mathrm{E}+01 \\
.904 \mathrm{E}+01 \\
.123 \mathrm{E}+02 \\
.290 \mathrm{E}+00 \\
.272 \mathrm{E}-02\end{array}$} & & \\
\hline & $\begin{array}{l}\text { LA SUPE } \\
\text { SOLUCIO } \\
\text { FESO4TU }\end{array}$ & & $\begin{array}{r}\text { AG+ } \\
.1 \mathrm{E}-12 \\
.4 \mathrm{E}-13 \\
.18 \mathrm{E}\end{array}$ & $\begin{array}{r}\text { AGT+ } \\
.5 \mathrm{E}-06 \\
.1 \mathrm{E}-06 \\
05\end{array}$ & $\begin{array}{l}\text { AGT2+ } \\
.5 \mathrm{E}-04 \\
.2 \mathrm{E}-04\end{array}$ & $\begin{array}{l}\text { AGT3+ } \\
.6 E-03 \\
.2 E-03\end{array}$ & $\begin{array}{r}\text { AGT4+ } \\
.5 E-04 \\
.2 E-04\end{array}$ & $\begin{array}{r}\mathrm{AG} 2 \mathrm{~T} 3+2 \\
.4 \mathrm{E}-02 \\
.4 \mathrm{E}-03\end{array}$ & $\begin{array}{l}\text { AG2T6+2 } \\
.63 \mathrm{E}-04 \\
.67 \mathrm{E}-05\end{array}$ \\
\hline
\end{tabular}

DIFUSIVIDAD EFECTIVA DE FE+3 $=1.372636606649 \mathrm{E}-9$ 


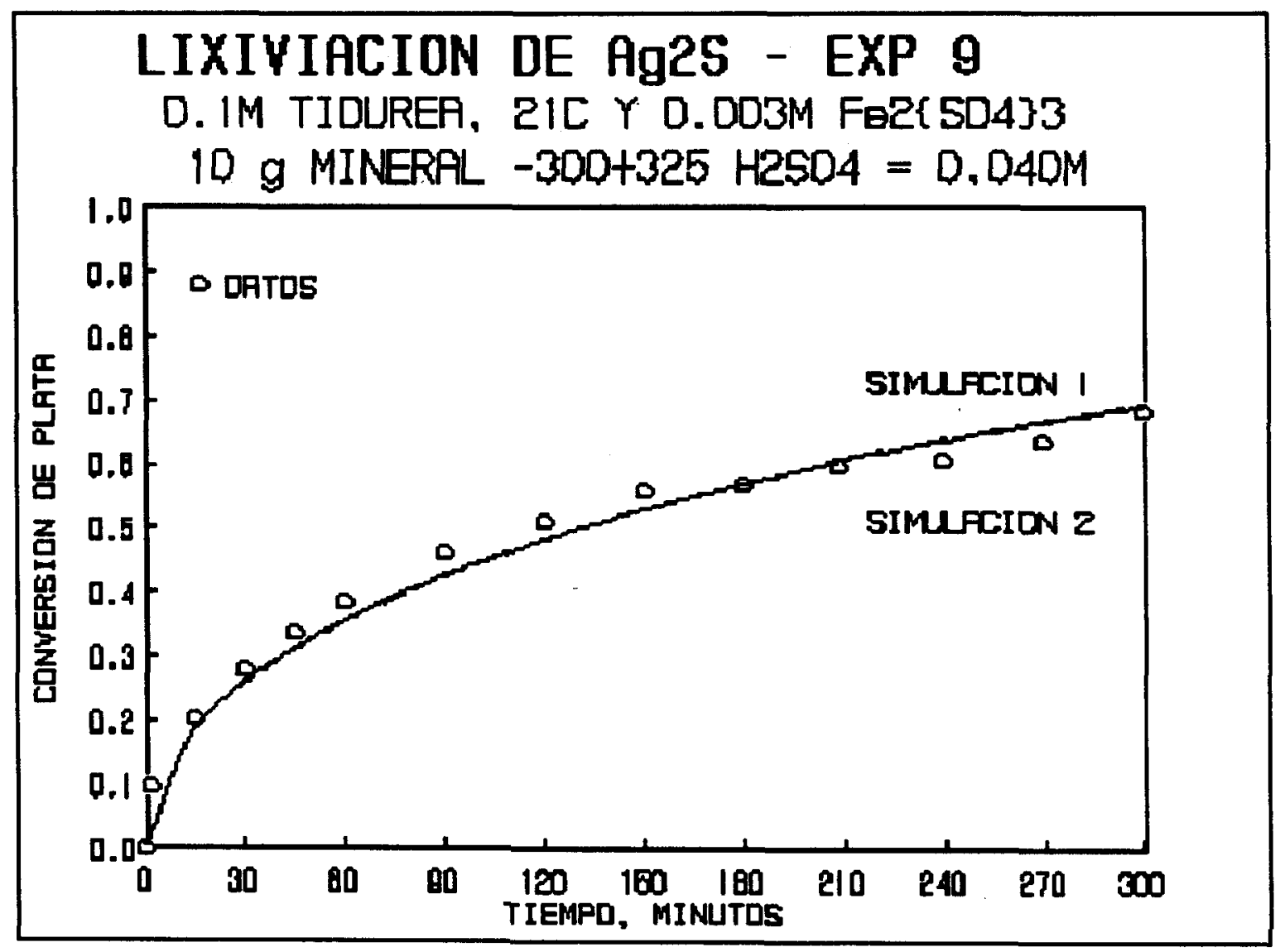




\section{EXPERIMENTO 10}

CONDICIONES :

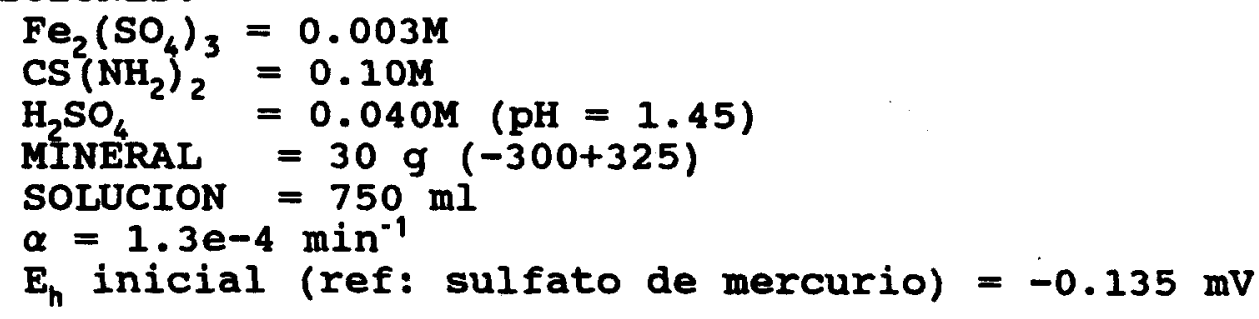

TIEMPO, MUESTRA, LECTURA, PPM CONVERSION minutos ml $\mathrm{Ag}$ Ag $\mathrm{Cu}$

$$
\mathbf{E}_{h^{\prime}}
$$

$\begin{array}{rrrrrrr}1.5 & 12 . & 19.5 & 5.6 & .0653 & .044 & -.211 \\ 17.0 & 6 . & 61.5 & 13.3 & .2036 & .105 & -.213 \\ 30.0 & 6 . & 75.0 & 15.0 & .2477 & .118 & -.214 \\ 45.0 & 6 . & 95.4 & 16.2 & .3138 & .127 & -.215 \\ 60.0 & 6 . & 97.5 & 17.5 & .3206 & .137 & -.216 \\ 90.0 & 6 . & 112.5 & 20.0 & .3684 & .156 & -.218 \\ 120.0 & 6 . & 130.0 & 20.0 & .4237 & .156 & -.219 \\ 145.0 & 6 . & 138.3 & 20.0 & .4497 & .156 & -.220 \\ 150.0 & 6 . & 140.0 & 20.0 & .4550 & .156 & -.220 \\ 180.0 & 6 . & 152.5 & 22.5 & .4935 & .174 & -.221 \\ 210.0 & 6 . & 162.5 & 22.5 & .5240 & .174 & -.222 \\ 240.0 & 6 . & 175.0 & 22.5 & .5618 & .174 & -.223 \\ 270.0 & 6 . & 177.5 & 22.5 & .5693 & .174 & -.224 \\ 295.0 & 6 . & 183.7 & 22.5 & .5878 & .174 & -.225 \\ 300.0 & 6 . & 185.0 & 22.5 & .5916 & .174 & -.225\end{array}$

RESIDUO DE Ag $=3.05 \mathrm{~kg} /$ ton

RESIDUO DE Cu $=2.60 \mathrm{~kg} /$ ton

BALANCE METALURGICO DE Ag $=7.47 \mathrm{~kg} / \mathrm{ton}$

BALANCE METALURGICO DE Cu $=3.15 \mathrm{~kg} /$ ton 


\section{Apéndice D - Experimento 10}

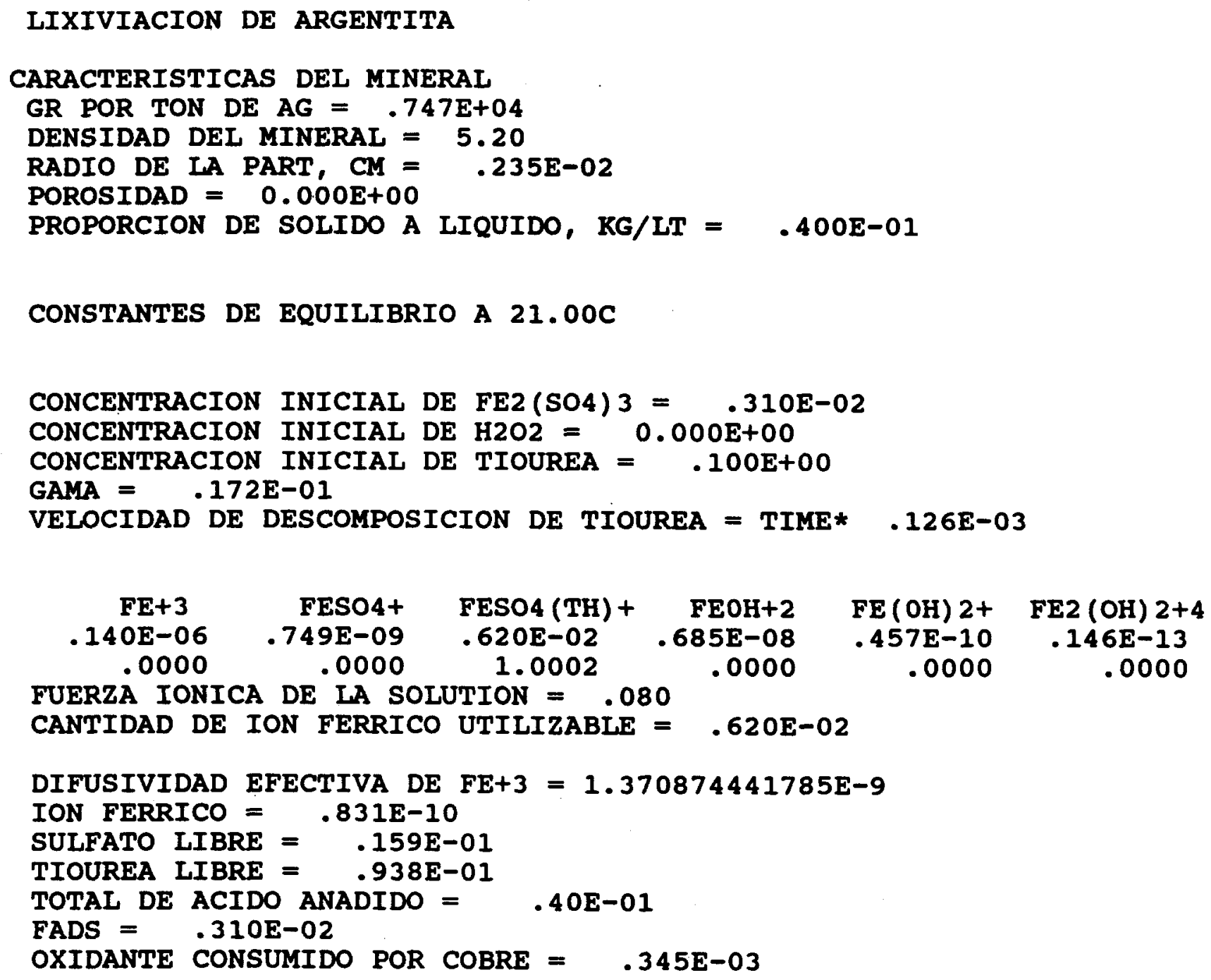

\section{PERFILES ADIMENSIONALES}

TIEMPO DE AJUSTE $=180$.

Ag2T6+2 $=.0001777251719128$ LIMITE $=.0000418526892699$

PRECIPITACION DE Ag2SO4 . 3Tu.H2O

DIFUSUVIDAD EFECTIVA $=1.115477458487 \mathrm{E}-9$

PH REAL $=1.450476826062$

$\begin{array}{lllll}\mathrm{Fe}^{3+} & .233 \mathrm{E}-08 & .542 \mathrm{E}-08 & .110 \mathrm{E}-07 & .128 \mathrm{E}-07 \\ \mathrm{SO}_{4}{ }^{2-} & .290 \mathrm{E}+01 & .282 \mathrm{E}+01 & .262 \mathrm{E}+01 & .256 \mathrm{E}+01 \\ \mathrm{H}^{+} & .780 \mathrm{E}+01 & .809 \mathrm{E}+01 & .890 \mathrm{E}+01 & .917 \mathrm{E}+01 \\ \mathrm{Tu}^{2+} & .150 \mathrm{E}+02 & .151 \mathrm{E}+02 & .153 \mathrm{E}+02 & .154 \mathrm{E}+02 \\ \mathrm{Fe}^{2+} & .268 \mathrm{E}+00 & .274 \mathrm{E}+00 & .288 \mathrm{E}+00 & .292 \mathrm{E}+00 \\ \mathrm{AgTu}_{2}{ }^{+} & .113 \mathrm{E}-01 & .990 \mathrm{E}-02 & .468 \mathrm{E}-02 & .584 \mathrm{E}-04\end{array}$


Apéndice D - Experimento 10

$\begin{array}{llrrrrrrr} & \text { AGt } & \text { AGT+ } & \text { AGT2+ } & \text { AGT3+ } & \text { AGT4+ } & \text { AG2T3+2 } & \text { AG2T6+2 } \\ \text { EN LA SUPER } & .1 \mathrm{E}-12 & .5 \mathrm{E}-06 & .7 \mathrm{E}-04 & .9 \mathrm{E}-03 & .1 \mathrm{E}-03 & .5 \mathrm{E}-02 & .17 \mathrm{E}-03 \\ \text { EN SOLUCION } & .5 \mathrm{E}-15 & .3 \mathrm{E}-08 & .4 \mathrm{E}-06 & .5 \mathrm{E}-05 & .5 \mathrm{E}-06 & .1 \mathrm{E}-06 & .48 \mathrm{E}-08\end{array}$

\begin{tabular}{|c|c|c|c|c|c|c|c|c|c|}
\hline $\begin{array}{r}\text { ADIM. } \\
.0 \\
.0 \\
.0 \\
.0 \\
.1 \\
.1 \\
.2 \\
.2 \\
.3\end{array}$ & $\begin{array}{l}\text { TIEM } \\
002 \\
027 \\
072 \\
087 \\
102 \\
152 \\
202 \\
252 \\
302\end{array}$ & 更 & $\begin{array}{l}\mathrm{RC} / \\
.994 \mathrm{E} \\
.976 \mathrm{E} \\
.960 \mathrm{E} \\
.956 \mathrm{E} \\
.953 \mathrm{E} \\
.939 \mathrm{E} \\
.931 \mathrm{E} \\
.925 \mathrm{E} \\
.919 \mathrm{E}\end{array}$ & $\begin{array}{l}R P \\
+00 \\
+00 \\
+00 \\
+00 \\
+00 \\
+00 \\
+00 \\
+00 \\
+00\end{array}$ & $\begin{array}{l}\text { IEMPO, MIN } \\
.130 E+00 \\
.219 E+01 \\
.591 E+01 \\
.714 E+01 \\
.838 E+01 \\
.125 E+02 \\
.166 E+02 \\
.208 E+02 \\
.249 E+02\end{array}$ & $\begin{array}{r}\text { CONV } \\
.15 \\
.64 \\
.10 \\
.11 \\
.12 \\
.15 \\
.17 \\
.19 \\
.21\end{array}$ & $\begin{array}{l}\text { SION } \\
-01 \\
-01 \\
+00 \\
+00 \\
+00 \\
+00 \\
+00 \\
+00 \\
+00\end{array}$ & $\begin{array}{l}\text { PH NUET } \\
1.45 \\
1.45 \\
1.45 \\
1.45 \\
1.45 \\
1.45 \\
1.45 \\
1.45 \\
1.45\end{array}$ & \\
\hline $\begin{array}{l}\mathrm{Fe}^{3+} \\
\mathrm{SO}_{4}{ }^{2-} \\
\mathrm{H}^{+} \\
\mathrm{Tu} \\
\mathrm{Fe}^{2+} \\
\mathrm{AgTu}_{2}^{+}\end{array}$ & & $\begin{array}{l}.257 \\
.287 \\
.786 \\
.149 \\
.270 \\
.109\end{array}$ & $\begin{array}{l}E-08 \\
E+01 \\
E+01 \\
E+02 \\
E+00 \\
E-01\end{array}$ & $\begin{array}{l}.548 E-08 \\
.280 E+01 \\
.814 E+01 \\
.150 E+02 \\
.275 E+00 \\
.957 E-02\end{array}$ & $\begin{array}{ll} & .105 \mathrm{E}-07 \\
1 & .263 \mathrm{E}+01 \\
1 & .885 \mathrm{E}+01 \\
2 & .151 \mathrm{E}+02 \\
0 & .287 \mathrm{E}+00 \\
2 & .501 \mathrm{E}-02\end{array}$ & $\begin{array}{l}.121 \\
.258 \\
.909 \\
.152 \\
.291 \\
.221\end{array}$ & $\begin{array}{l}-07 \\
01 \\
-01 \\
-02 \\
-00 \\
-02\end{array}$ & & \\
\hline EN LA & 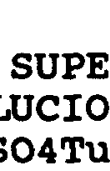 & & $\begin{array}{r}\text { AG+ } \\
1 \mathrm{E}-12 \\
2 \mathrm{E}-13 \\
.75 \mathrm{E}\end{array}$ & $\begin{array}{r}\text { AGT+ } \\
.5 \mathrm{E}-06 \\
.1 \mathrm{E}-06\end{array}$ & $\begin{array}{r}\text { AGT2+ } \\
.7 \mathrm{E}-04 \\
.1 \mathrm{E}-04\end{array}$ & $\begin{array}{r}\text { AGT3+ } \\
.9 E-03 \\
.2 E-03\end{array}$ & $\begin{array}{r}\text { AGT4+ } \\
.9 E-04 \\
.2 E-04\end{array}$ & $\begin{array}{r}\text { AG2T } 3+2 \\
.5 \mathrm{E}-02 \\
.2 \mathrm{E}-03\end{array}$ & $\begin{array}{r}\text { AG2T6+2 } \\
.16 \mathrm{E}-03 \\
.67 \mathrm{E}-05\end{array}$ \\
\hline
\end{tabular}

$\begin{array}{cc}\text { ADIM. TIEMPO } & \text { RC/RP } \\ .452 & .898 \mathrm{E}+00 \\ .552 & .889 \mathrm{E}+00 \\ .652 & .881 \mathrm{E}+00 \\ .752 & .873 \mathrm{E}+00 \\ .852 & .866 \mathrm{E}+00 \\ 1.002 & .853 \mathrm{E}+00 \\ 1.152 & .844 \mathrm{E}+00 \\ 1.302 & .835 \mathrm{E}+00 \\ 1.452 & .826 \mathrm{E}+00 \\ 1.602 & .819 \mathrm{E}+00 \\ 1.902 & .798 \mathrm{E}+00 \\ 2.202 & .785 \mathrm{E}+00 \\ 2.502 & .772 \mathrm{E}+00 \\ 2.802 & .761 \mathrm{E}+00 \\ 3.102 & .749 \mathrm{E}+00 \\ 3.602 & .726 \mathrm{E}+00 \\ 4.102 & .711 \mathrm{E}+00 \\ 4.602 & .696 \mathrm{E}+00 \\ 5.102 & .682 \mathrm{E}+00\end{array}$

TIEMPO, MIN CONVERSION

$.373 \mathrm{E}+02$

$.455 \mathrm{E}+02$

$.538 \mathrm{E}+02$

$.620 \mathrm{E}+02$

$.703 \mathrm{E}+02$

$.826 \mathrm{E}+02$

$.950 \mathrm{E}+02$

$.107 \mathrm{E}+03$

$.120 \mathrm{E}+03$

$.132 \mathrm{E}+03$

$.157 \mathrm{E}+03$

$.182 \mathrm{E}+03$

$.206 \mathrm{E}+03$

$.231 E+03$

$.256 \mathrm{E}+03$

$.297 \mathrm{E}+03$

$.338 \mathrm{E}+03$

$.380 \mathrm{E}+03$

$.421 \mathrm{E}+03$
$.254 \mathrm{E}+00$

$.278 \mathrm{E}+00$

$.299 \mathrm{E}+00$

$.319 \mathrm{E}+00$

$.337 \mathrm{E}+00$

$.361 \mathrm{E}+00$

$.382 \mathrm{E}+00$

$.402 \mathrm{E}+00$

$.421 E+00$

$.438 \mathrm{E}+00$

$.469 \mathrm{E}+00$

$.496 \mathrm{E}+00$

$.520 E+00$

$.543 \mathrm{E}+00$

$.563 \mathrm{E}+00$

$.593 E+00$

$.620 \mathrm{E}+00$

$.644 \mathrm{E}+00$

$.666 \mathrm{E}+00$
PH NUEVO

1.46

1.46

1.46

1.46

1.46

1.46

1.46

1.46

1.46

1.46

1.46

1.46

1.46

1.46

1.46

1.46

1.46

1.46

1.46 
Apéndice D - Experimento 10

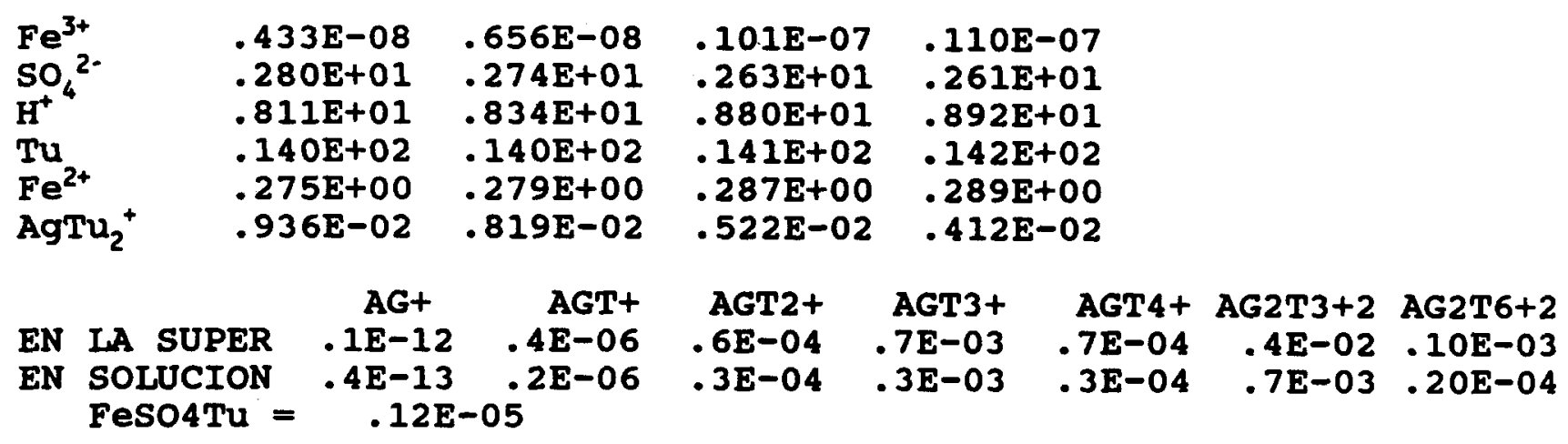




\section{Apéndice D - Experimento 10}

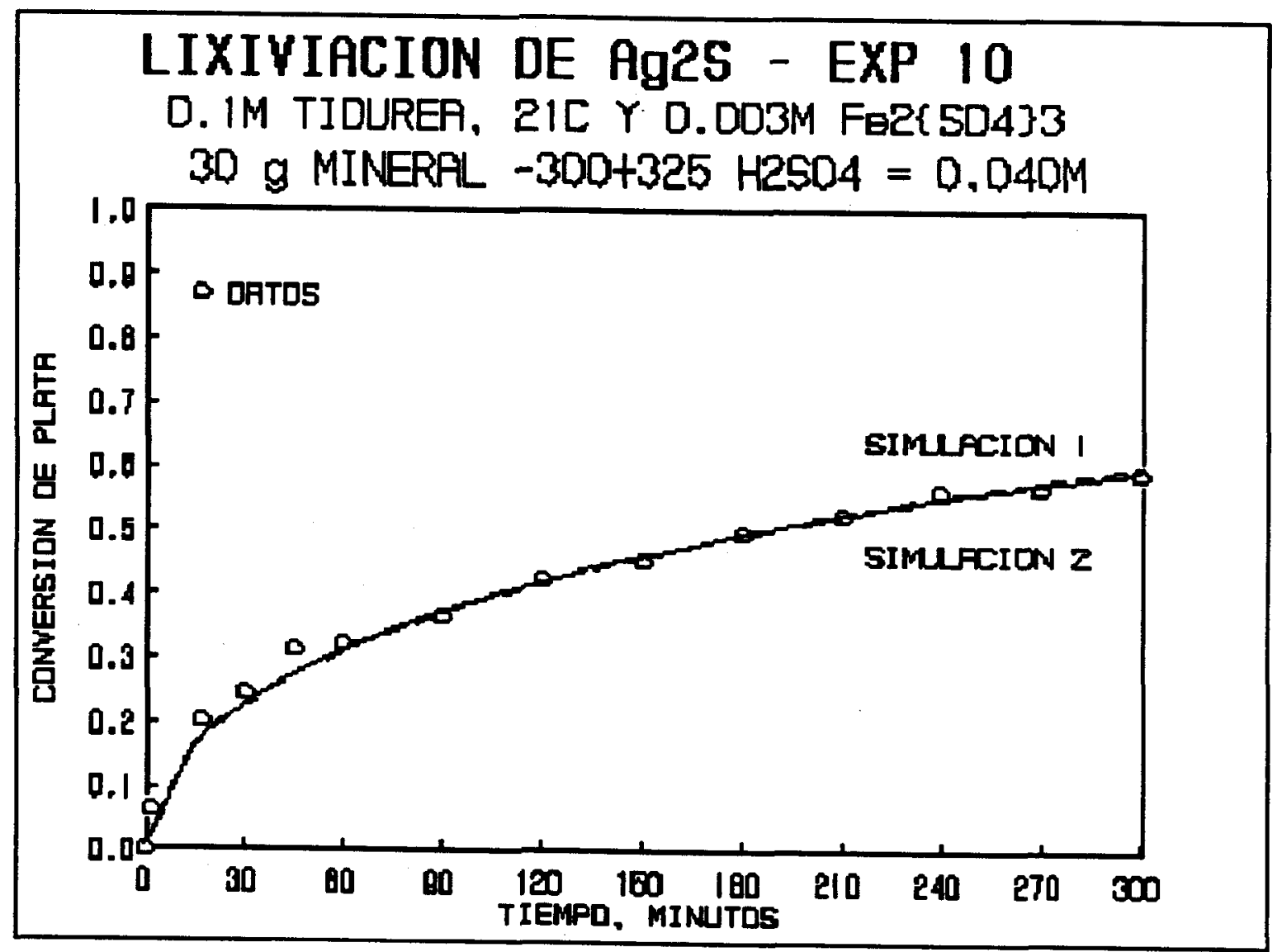




\section{EXPERIMENTO 11}

CONDICIONES :

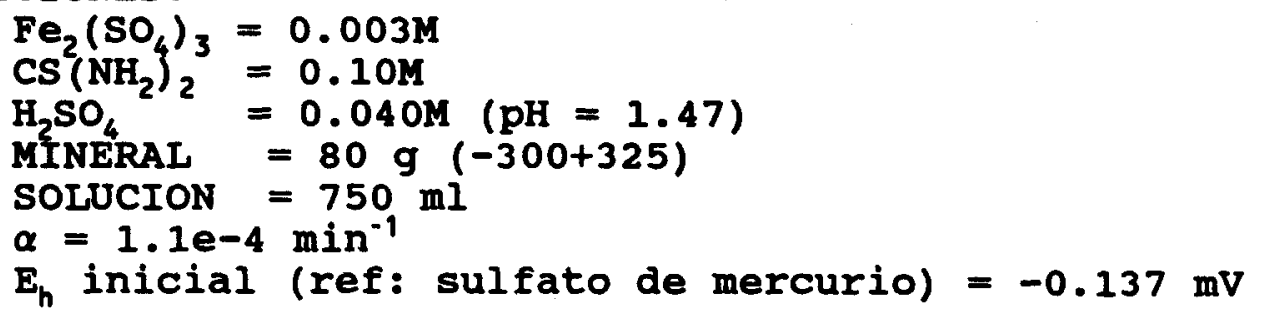

\begin{tabular}{|c|c|c|c|c|c|c|}
\hline $\begin{array}{l}\text { TIEMPO, } \\
\text { minutos }\end{array}$ & $\begin{array}{c}\text { MUESTRA, } \\
\mathrm{ml}\end{array}$ & $\begin{array}{l}\text { LECTURA } \\
\text { Ag }\end{array}$ & t, $\mathrm{Cu}$ & $\begin{array}{r}\text { CON } \\
\mathrm{Ag}\end{array}$ & $\begin{array}{l}\text { SION } \\
\text { Cu }\end{array}$ & $\begin{array}{l}E_{h \prime} \\
m V\end{array}$ \\
\hline $\begin{array}{r}1.0 \\
15.0 \\
30.0 \\
45.0 \\
60.0 \\
90.0 \\
120.0 \\
146.0 \\
151.0 \\
180.0 \\
210.0 \\
240.0 \\
270.0 \\
295.0 \\
300.0\end{array}$ & $\begin{array}{l}12 . \\
6 . \\
6 . \\
6 . \\
6 . \\
6 . \\
6 . \\
6 . \\
6 . \\
6 . \\
6 . \\
6 . \\
6 . \\
6 . \\
6 .\end{array}$ & $\begin{array}{r}37.5 \\
115.5 \\
150.0 \\
175.0 \\
205.0 \\
235.0 \\
275.0 \\
298.5 \\
305.0 \\
325.0 \\
345.0 \\
360.0 \\
375.0 \\
385.0 \\
385.0\end{array}$ & $\begin{array}{l}12.5 \\
27.5 \\
32.5 \\
37.5 \\
40.0 \\
45.0 \\
50.0 \\
50.0 \\
50.0 \\
55.0 \\
55.0 \\
55.0 \\
55.0 \\
60.0 \\
60.0\end{array}$ & $\begin{array}{l}.045 \\
.136 \\
.177 \\
.206 \\
.240 \\
.274 \\
.319 \\
.346 \\
.353 \\
.375 \\
.396 \\
.413 \\
.429 \\
.439 \\
.439\end{array}$ & $\begin{array}{l}.036 \\
.079 \\
.093 \\
.107 \\
.114 \\
.128 \\
.141 \\
.141 \\
.141 \\
.155 \\
.155 \\
.155 \\
.155 \\
.167 \\
.167\end{array}$ & $\begin{array}{l}-.210 \\
-.212 \\
-.213 \\
-.213 \\
-.214 \\
-.215 \\
-.217 \\
-.218 \\
-.218 \\
-.220 \\
-.221 \\
-.222 \\
-.223 \\
-.225 \\
-.225\end{array}$ \\
\hline
\end{tabular}

RESIDUO DE Ag $=4.40 \mathrm{~kg} / \mathrm{ton}$

RESIDUO DE $\mathrm{Cu}=2.70 \mathrm{~kg} /$ ton

BALANCE METALURGICO DE Ag $=7.85 \mathrm{~kg} /$ ton

BALANCE METALURGICO DE $\mathrm{Cu}=3.24 \mathrm{~kg} / \mathrm{ton}$ 
Apéndice D - Experimento 11

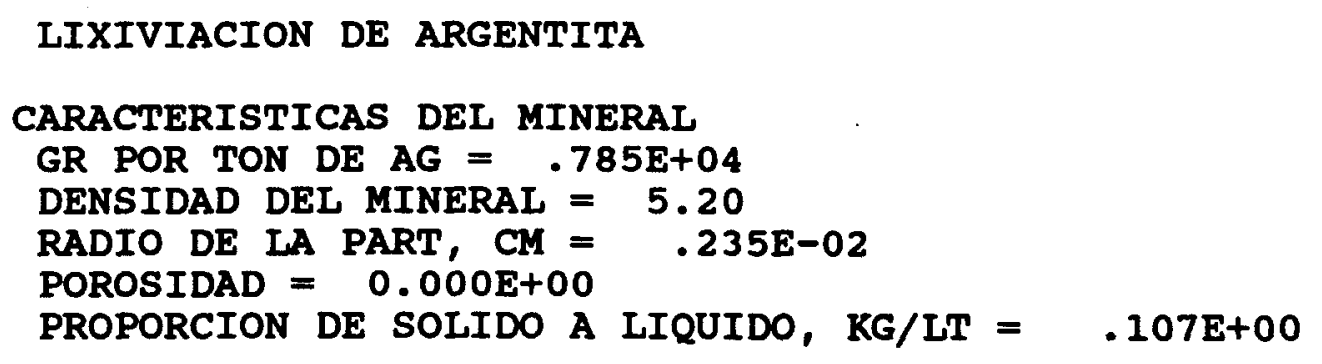

\section{PERFILES ADIMENSIONALES}

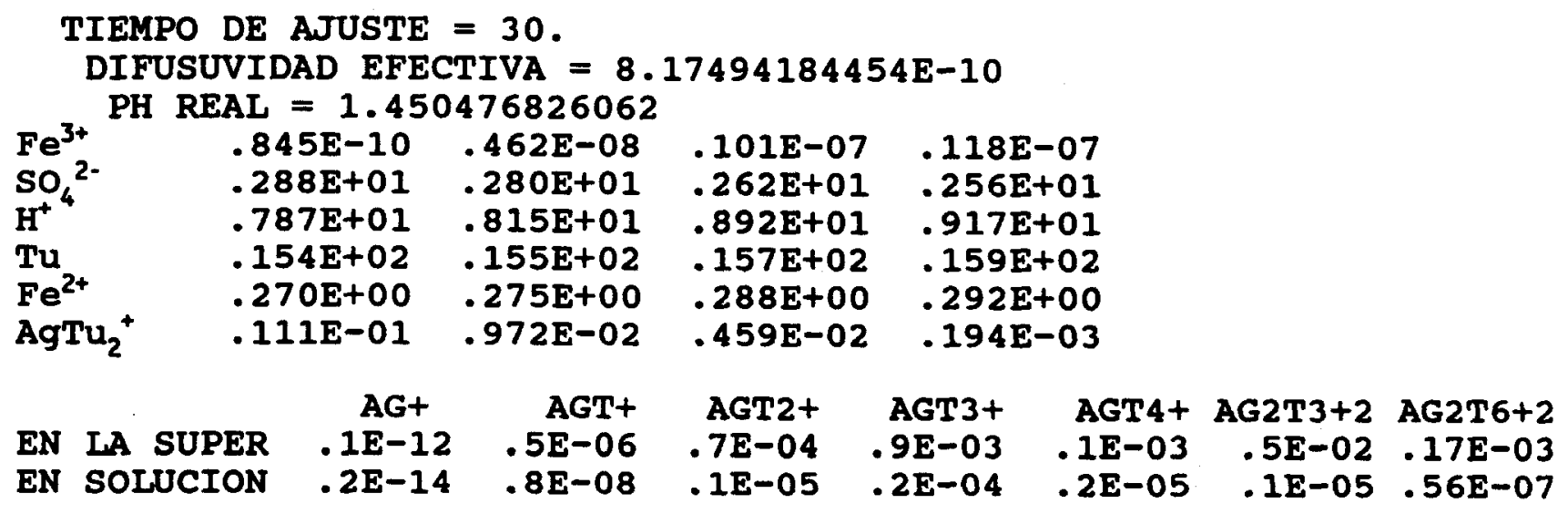


Apéndice D - Experimento 11

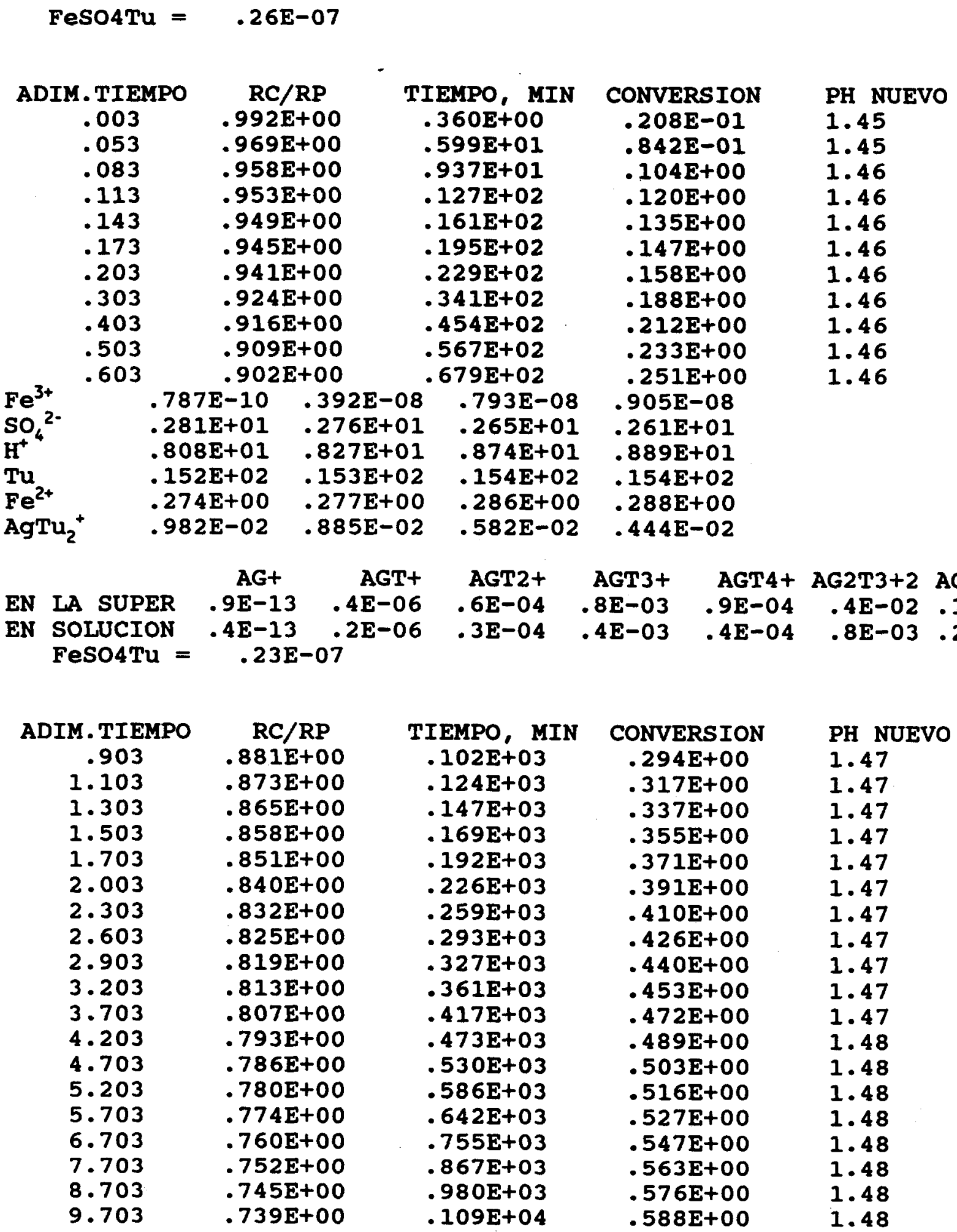


Apéndice D - Experimento 11

$\begin{array}{lllll}\mathrm{Fe}^{3+} & .811 \mathrm{E}-10 & .225 \mathrm{E}-08 & .404 \mathrm{E}-08 & .444 \mathrm{E}-08 \\ \mathrm{SO}_{4}{ }^{2-} & .271 \mathrm{E}+01 & .270 \mathrm{E}+01 & .268 \mathrm{E}+01 & .267 \mathrm{E}+01 \\ \mathrm{H}^{+} & .838 \mathrm{E}+01 & .843 \mathrm{E}+01 & .852 \mathrm{E}+01 & .855 \mathrm{E}+01 \\ \mathrm{Tu} & .133 \mathrm{E}+02 & .133 \mathrm{E}+02 & .133 \mathrm{E}+02 & .133 \mathrm{E}+02 \\ \mathrm{Fe}^{2+} & .281 \mathrm{E}+00 & .281 \mathrm{E}+00 & .283 \mathrm{E}+00 & .283 \mathrm{E}+00 \\ \mathrm{AgTu}_{2}{ }^{+} & .754 \mathrm{E}-02 & .729 \mathrm{E}-02 & .673 \mathrm{E}-02 & .655 \mathrm{E}-02\end{array}$

$\begin{array}{llcrrrrrr} & & \text { AGt } & \text { AGT+ } & \text { AGT2+ } & \text { AGT3+ } & \text { AGT4+ } & \text { AG2T3+2 } & \text { AG2T6+2 } \\ \text { EN LA SUPER } & .9 \mathrm{E}-13 & .4 \mathrm{E}-06 & .5 \mathrm{E}-04 & .5 \mathrm{E}-03 & .5 \mathrm{E}-04 & .3 \mathrm{E}-02 & .59 \mathrm{E}-04 \\ \text { EN SOLUCION } & .8 \mathrm{E}-13 & .3 \mathrm{E}-06 & .4 \mathrm{E}-04 & .5 \mathrm{E}-03 & .5 \mathrm{E}-04 & .2 \mathrm{E}-02 & .45 \mathrm{E}-04 \\ \text { FeSO4TU }= & .20 \mathrm{E}-07 & & & & & & \end{array}$

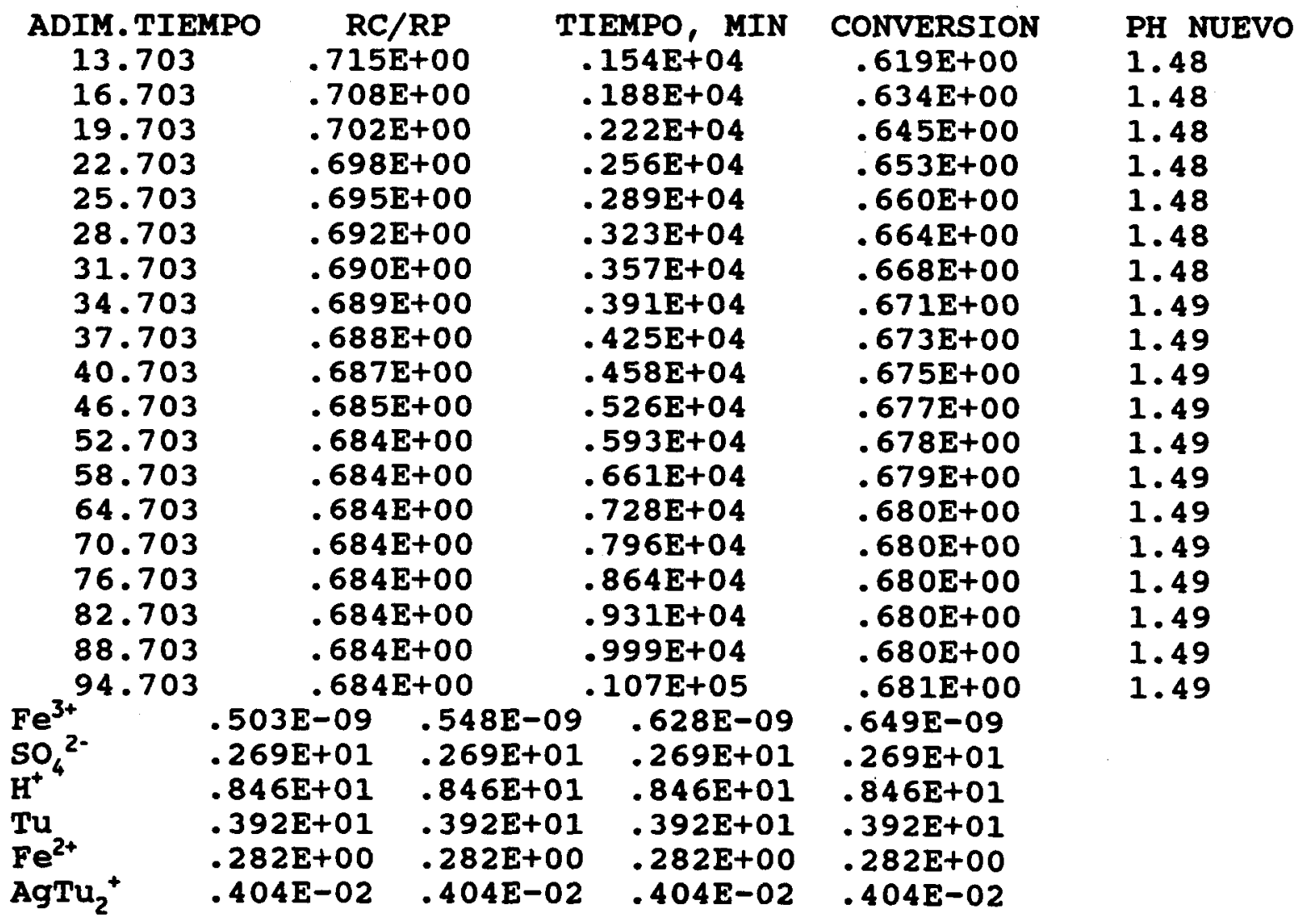

$\begin{array}{llcrrrrrr} & \text { AG+ } & \text { AGT+ } & \text { AGT2+ } & \text { AGT3+ } & \text { AGT4+ } & \text { AG2T3+2 } & \text { AG2T6+2 } \\ \text { EN LA SUPER } & .6 \mathrm{E}-12 & .7 \mathrm{E}-06 & .3 \mathrm{E}-04 & .9 \mathrm{E}-04 & .2 \mathrm{E}-05 & .3 \mathrm{E}-02 & .15 \mathrm{E}-05 \\ \text { EN SOLUCION } & .6 \mathrm{E}-12 & .7 \mathrm{E}-06 & .3 \mathrm{E}-04 & .9 \mathrm{E}-04 & .2 \mathrm{E}-05 & .3 \mathrm{E}-02 & .15 \mathrm{E}-05\end{array}$

$\begin{array}{lcccl}\text { ADIM.TIEMPO } & \text { RC/RP } & \text { TIEMPO, MIN } & \text { CONVERSION } & \text { PH NUEVO } \\ 110.703 & .684 \mathrm{E}+00 & .125 \mathrm{E}+05 & .681 \mathrm{E}+00 & 1.49 \\ 120.703 & .684 \mathrm{E}+00 & .136 \mathrm{E}+05 & .681 \mathrm{E}+00 & 1.49\end{array}$


Apéndice D - Experimento 11

$\begin{array}{lllll}130.703 & .684 \mathrm{E}+00 & .147 \mathrm{E}+05 & .681 \mathrm{E}+00 & 1.49 \\ 140.703 & .684 \mathrm{E}+00 & .158 \mathrm{E}+05 & .681 \mathrm{E}+00 & 1.49 \\ 150.703 & .684 \mathrm{E}+00 & .170 \mathrm{E}+05 & .681 \mathrm{E}+00 & 1.49 \\ 160.703 & .684 \mathrm{E}+00 & .181 \mathrm{E}+05 & .681 \mathrm{E}+00 & 1.49 \\ 170.703 & .684 \mathrm{E}+00 & .192 \mathrm{E}+05 & .680 \mathrm{E}+00 & 1.49 \\ 180.703 & .684 \mathrm{E}+00 & .203 \mathrm{E}+05 & .680 \mathrm{E}+00 & 1.49 \\ 190.703 & .684 \mathrm{E}+00 & .215 \mathrm{E}+05 & .680 \mathrm{E}+00 & 1.49 \\ 200.703 & .684 \mathrm{E}+00 & .226 \mathrm{E}+05 & .680 \mathrm{E}+00 & 1.49\end{array}$




\section{Apéndice D - Experimento 11}

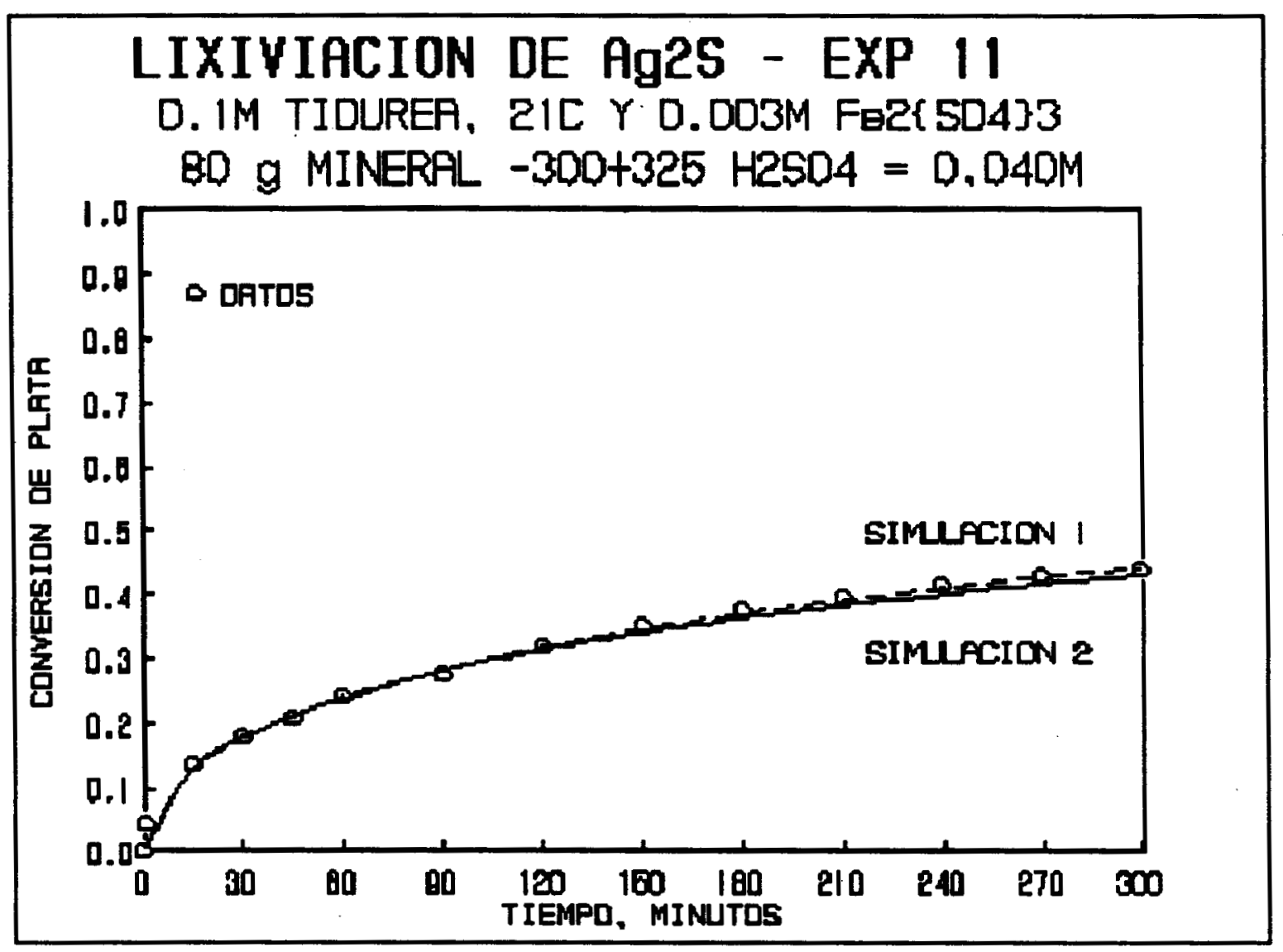




\section{EXPERIMENTO 12}

CONDICIONES :

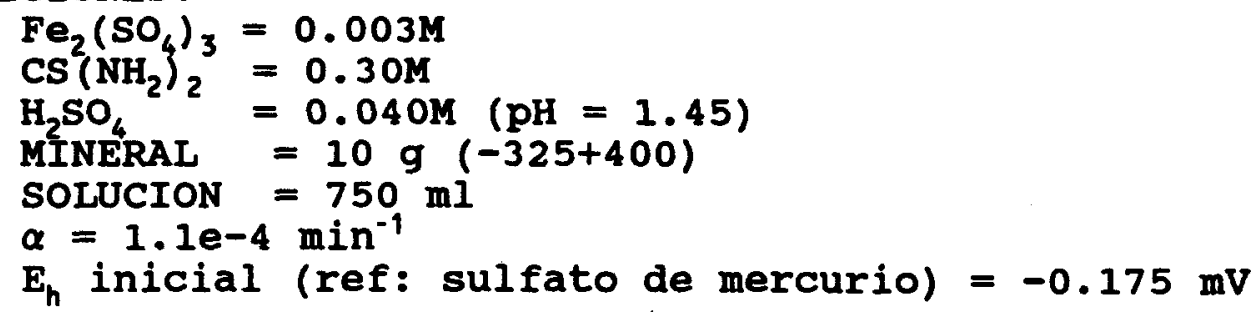

\begin{tabular}{rcccccc} 
TIEMPO, & MUESTRA, & \multicolumn{2}{c}{ LECTURA, PPM } & \multicolumn{2}{c}{ CONVERSION } & $E_{h^{\prime}}$ \\
minutos & ml & Ag & Cu & Ag & Cu & mV \\
1.5 & 12. & 14.5 & 3.6 & .128 & .078 & -.235 \\
15.0 & 6. & 33.5 & 6.4 & .293 & .138 & -.243 \\
31.0 & 6. & 38.4 & 7.5 & .336 & .162 & -.245 \\
45.0 & 6. & 41.4 & 7.9 & .361 & .170 & -.247 \\
60.0 & 6. & 44.0 & 8.1 & .383 & .174 & -.248 \\
90.0 & 6. & 48.4 & 8.3 & .420 & .178 & -.249 \\
120.0 & 6. & 51.7 & 8.4 & .448 & .180 & -.250 \\
146.0 & 6. & 52.8 & 8.5 & .457 & .182 & -.252 \\
151.0 & 6. & 53.9 & 8.5 & .466 & .182 & -.252 \\
180.0 & 6. & 57.2 & 8.6 & .493 & .184 & -.253 \\
210.0 & 6. & 58.3 & 8.7 & .502 & .186 & -.254 \\
240.0 & 6. & 61.6 & 8.7 & .528 & .186 & -.256 \\
270.0 & 6. & 63.8 & 8.8 & .545 & .188 & -.257 \\
295.0 & 6. & 64.9 & 8.8 & .554 & .188 & -.258 \\
300.0 & 6. & 67.1 & 8.8 & .571 & .188 & -.258
\end{tabular}

RESIDUO DE Ag $=3.64 \mathrm{~kg} /$ ton

RESIDUO DE Cu $=2.80 \mathrm{~kg} /$ ton

BALANCE METALURGICO DE Ag $=8.49 \mathrm{~kg} /$ ton

BALANCE METALURGICO DE Cu $=3.45 \mathrm{~kg} /$ ton 
Apéndice D - Experimento 12

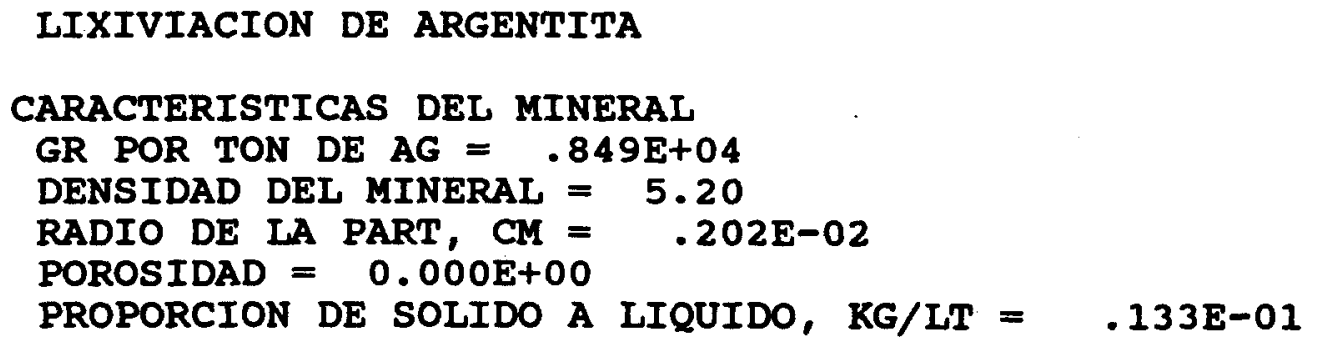

\section{PERFILES ADIMENSIONALES}

TIEMPO DE AJUSTE $=180$.

Ag2T6+2 $=.001597142685073$ LIMITE $=.0001352587943039$

PRECIPITACION DE Ag2SO4 . 3Tu.H2O

DIFUSUVIDAD EFECTIVA $=1.768725395291 \mathrm{E}-8$

PH REAL $=1.450476980615$

$\begin{array}{lllll}\mathrm{Fe}^{3+} & .154 \mathrm{E}-08 & .212 \mathrm{E}-08 & .370 \mathrm{E}-08 & .428 \mathrm{E}-08 \\ \mathrm{SO}_{4}{ }^{2-} & .269 \mathrm{E}+01 & .266 \mathrm{E}+01 & .259 \mathrm{E}+01 & .256 \mathrm{E}+01 \\ \mathrm{H}^{+} & .908 \mathrm{E}+01 & .910 \mathrm{E}+01 & .916 \mathrm{E}+01 & .918 \mathrm{E}+01 \\ \mathrm{Tu}^{2+} & .426 \mathrm{E}+02 & .436 \mathrm{E}+02 & .464 \mathrm{E}+02 & .474 \mathrm{E}+02 \\ \mathrm{Fe}^{2+} & .292 \mathrm{E}+00 & .292 \mathrm{E}+00 & .292 \mathrm{E}+00 & .292 \mathrm{E}+00 \\ \mathrm{AgTu}_{2}{ }^{+} & .257 \mathrm{E}-03 & .204 \mathrm{E}-03 & .582 \mathrm{E}-04 & .500 \mathrm{E}-05\end{array}$


Apéndice $D$ - Experimento 12

$\begin{array}{lcccccccc} & \text { AG+ } & \text { AGT+ } & \text { AGT2+ } & \text { AGT3+ } & \text { AGT4+ } & \text { AG2T3+2 } & \text { AG2T6+2 } \\ \text { EN IA SUPER } & .3 \mathrm{E}-15 & .4 \mathrm{E}-08 & .2 \mathrm{E}-05 & .6 \mathrm{E}-04 & .2 \mathrm{E}-04 & .1 \mathrm{E}-05 & .71 \mathrm{E}-06 \\ \text { EN SOLUCION } & .5 \mathrm{E}-17 & .7 \mathrm{E}-10 & .3 \mathrm{E}-07 & .1 \mathrm{E}-05 & .4 \mathrm{E}-06 & .3 \mathrm{E}-09 & .33 \mathrm{E}-09\end{array}$

\begin{tabular}{|c|c|c|c|c|}
\hline $\begin{array}{c}\text { ADIM. TIEMI } \\
.002 \\
.027 \\
.102\end{array}$ & $\begin{array}{r}R C \\
.994 \\
.991 \\
.985\end{array}$ & $\begin{array}{l}.0 \\
.00 \\
.00 \\
.00\end{array}$ & $\begin{array}{l}\text { ГIEMPO, MIN } \\
.606 \mathrm{E}-02 \\
.102 \mathrm{E}+00 \\
.391 \mathrm{E}+00\end{array}$ & $\begin{array}{c}\text { CONVERSION } \\
.178 \mathrm{E}-01 \\
.264 \mathrm{E}-01 \\
.422 \mathrm{E}-01\end{array}$ \\
\hline $\begin{array}{l}\mathrm{Fe}^{3+} \\
\mathrm{SO}_{4}^{2-} \\
\mathrm{H}^{+} \\
\mathrm{Tu}^{2+} \\
\mathrm{Fe}^{2+} \\
\mathrm{AgTu}_{2}^{+}\end{array}$ & $\begin{array}{l}398 E-08 \\
.258 E+01 \\
.909 E+01 \\
474 E+02 \\
291 E+00 \\
163 E-02\end{array}$ & $\begin{array}{l}.403 \mathrm{E}-08 \\
.257 \mathrm{E}+01 \\
.911 \mathrm{E}+01 \\
.474 \mathrm{E}+02 \\
.291 \mathrm{E}+00 \\
.134 \mathrm{E}-02\end{array}$ & $\begin{array}{ll}8 & .415 \mathrm{E}-08 \\
1 & .257 \mathrm{E}+01 \\
1 & .915 \mathrm{E}+01 \\
2 & .474 \mathrm{E}+02 \\
0 & .292 \mathrm{E}+00 \\
2 & .525 \mathrm{E}-03\end{array}$ & $\begin{array}{l}.419 E-08 \\
.256 E+01 \\
.916 E+01 \\
.475 E+02 \\
.292 E+00 \\
.208 E-03\end{array}$ \\
\hline
\end{tabular}

PH NUEVO

1.45

1.45

1.45

$\begin{array}{llcrrrrrr} & \text { AGt } & \text { AGT+ } & \text { AGT2+ } & \text { AGT3+ } & \text { AGT4+ } & \text { AG2T3+2 } & \text { AG2T6+2 } \\ \text { EN IA SUPER } & .2 \mathrm{E}-14 & .2 \mathrm{E}-07 & .1 \mathrm{E}-04 & .4 \mathrm{E}-03 & .1 \mathrm{E}-03 & .3 \mathrm{E}-04 & .35 \mathrm{E}-04 \\ \text { EN SOLUCION } & .2 \mathrm{E}-15 & .3 \mathrm{E}-08 & .1 \mathrm{E}-05 & .5 \mathrm{E}-04 & .2 \mathrm{E}-04 & .6 \mathrm{E}-06 & .58 \mathrm{E}-06\end{array}$

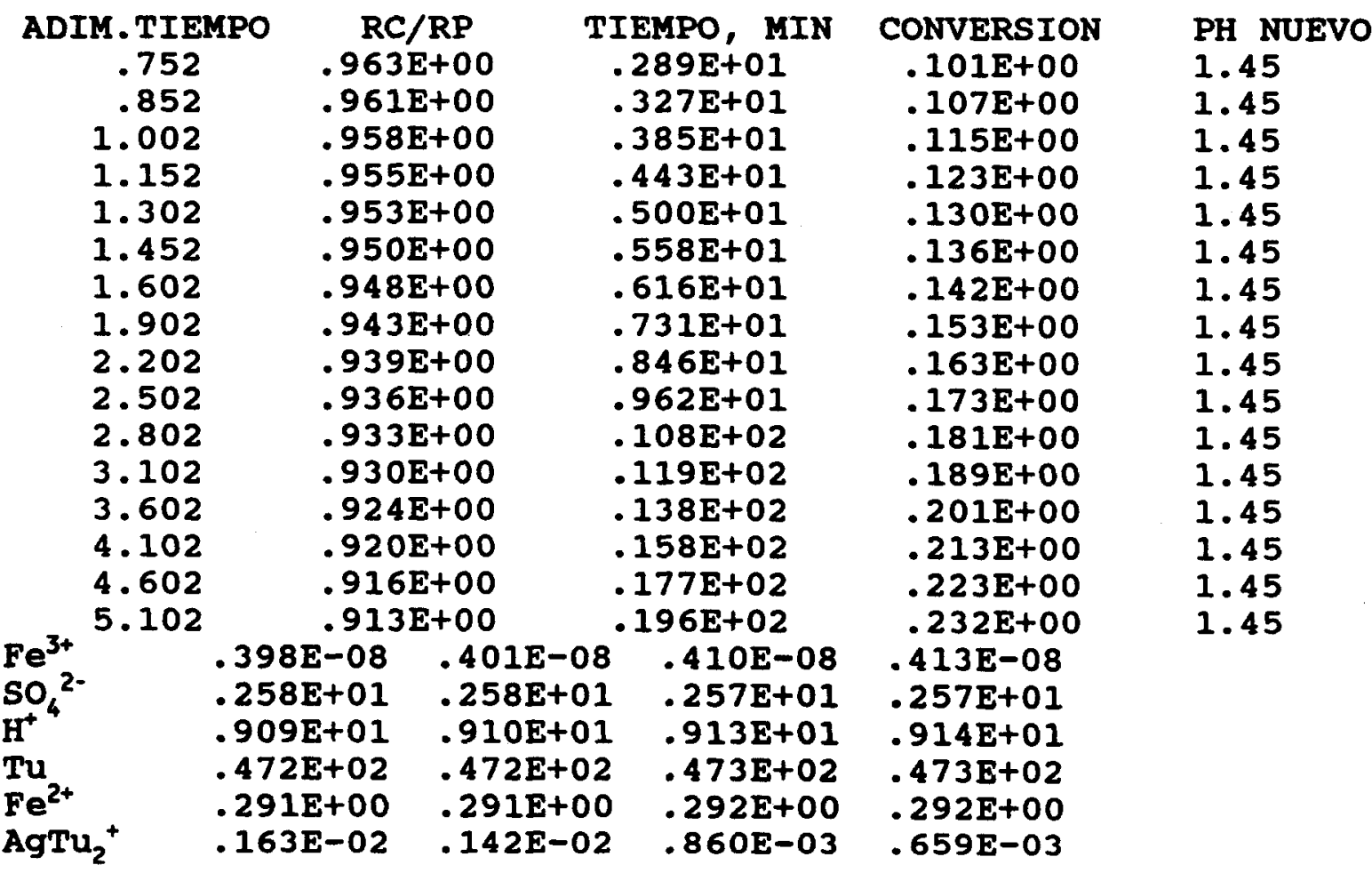


Apéndice D - Experimento 12

$\begin{array}{lccccccc} & \text { AGt } & \text { AGTt } & \text { AGT2+ } & \text { AGT3+ } & \text { AGT4+ } & \text { AG2T3+2 } & \text { AG2T6+2 } \\ \text { EN IA SUPER } & .2 \mathrm{E}-14 & .2 \mathrm{E}-07 & .1 \mathrm{E}-04 & .4 \mathrm{E}-03 & .1 \mathrm{E}-03 & .4 \mathrm{E}-04 & .35 \mathrm{E}-04 \\ \text { EN SOLUCION } & .6 \mathrm{E}-15 & .9 \mathrm{E}-08 & .4 \mathrm{E}-05 & .02 \mathrm{E}-03 & .6 \mathrm{E}-04 & .6 \mathrm{E}-05 & .57 \mathrm{E}-05\end{array}$

\begin{tabular}{|c|c|c|c|c|}
\hline $\begin{array}{c}\text { ADIM.TIEMI } \\
7.102 \\
8.602 \\
10.102 \\
11.602 \\
13.102 \\
14.602 \\
16.102 \\
17.602 \\
19.102 \\
20.602 \\
23.602 \\
26.602 \\
29.602 \\
32.602 \\
35.602 \\
38.602 \\
41.602 \\
44.602 \\
47.602\end{array}$ & IPO & \multicolumn{2}{|c|}{$\begin{array}{c}\mathrm{RC} / \mathrm{RP} \\
.895 \mathrm{E}+00 \\
.888 \mathrm{E}+00 \\
.881 \mathrm{E}+00 \\
.875 \mathrm{E}+00 \\
.869 \mathrm{E}+00 \\
.864 \mathrm{E}+00 \\
.859 \mathrm{E}+00 \\
.854 \mathrm{E}+00 \\
.850 \mathrm{E}+00 \\
.846 \mathrm{E}+00 \\
.835 \mathrm{E}+00 \\
.828 \mathrm{E}+00 \\
.822 \mathrm{E}+00 \\
.816 \mathrm{E}+00 \\
.811 \mathrm{E}+00 \\
.806 \mathrm{E}+00 \\
.801 \mathrm{E}+00 \\
.797 \mathrm{E}+00 \\
.793 \mathrm{E}+00\end{array}$} & 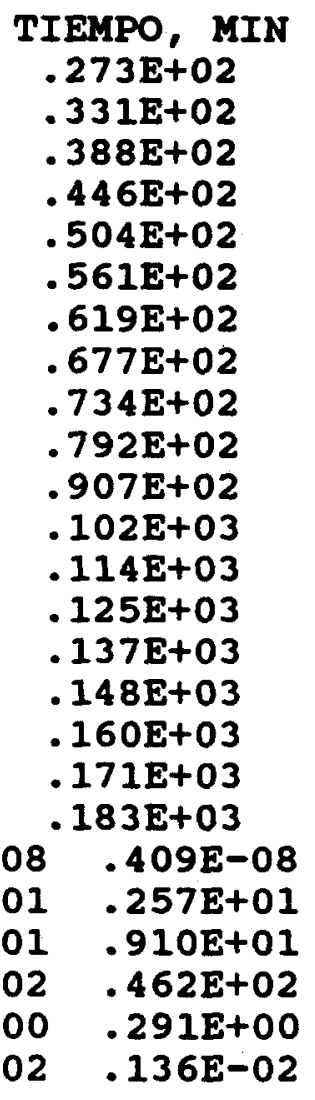 \\
\hline
\end{tabular}

CONVERSION
$.264 E+00$
$.284 E+00$
$.302 E+00$
$.317 E+00$
$.331 E+00$
$.344 E+00$
$.356 E+00$
$.367 E+00$
$.378 E+00$
$.387 E+00$
$.404 E+00$
$.420 E+00$
$.434 E+00$
$.446 E+00$
$.457 E+00$
$.468 E+00$
$.478 E+00$
$.487 E+00$
$.495 E+00$
$.410 E-08$
$.257 E+01$
$.910 E+01$
$.462 E+02$
$.291 E+00$
$.129 E-02$

CONVERSION

$.284 \mathrm{E}+00$

PH NUEVO

1.45

1.45

1.45

1.45

1.45

1.45

1.45

1.45

1.45

1.45

1.45

1.45

1.45

1.45

1.45

1.45

1.45

1.45

1.45

$\begin{array}{llcrrrrrr} & \text { AGt } & \text { AGT+ } & \text { AGT2+ } & \text { AGT3+ } & \text { AGT4+ } & \text { AG2T3+2 } & \text { AG2T6+2 } \\ \text { EN LA SUPER } & .2 \mathrm{E}-14 & .2 \mathrm{E}-07 & .1 \mathrm{E}-04 & .4 \mathrm{E}-03 & .1 \mathrm{E}-03 & .4 \mathrm{E}-04 & .35 \mathrm{E}-04 \\ \text { EN SOLUCION } & .1 \mathrm{E}-14 & .2 \mathrm{E}-07 & .8 \mathrm{E}-05 & .3 \mathrm{E}-03 & .1 \mathrm{E}-03 & .2 \mathrm{E}-04 & .21 \mathrm{E}-04\end{array}$

$\begin{array}{ccccl}\text { ADIM. TIEMPO } & \text { RC/RP } & \text { TIEMPO, MIN } & \text { CONVERSION } & \text { PH NUEVO } \\ 55.602 & .781 \mathrm{E}+00 & .214 \mathrm{E}+03 & .515 \mathrm{E}+00 & 1.45 \\ 60.602 & .775 \mathrm{E}+00 & .233 \mathrm{E}+03 & .526 \mathrm{E}+00 & 1.45 \\ 65.602 & .770 \mathrm{E}+00 & .252 \mathrm{E}+03 & .535 \mathrm{E}+00 & 1.45 \\ 70.602 & .766 \mathrm{E}+00 & .271 \mathrm{E}+03 & .544 \mathrm{E}+00 & 1.45 \\ 75.602 & .761 \mathrm{E}+00 & .291 \mathrm{E}+03 & .552 \mathrm{E}+00 & 1.45 \\ 80.602 & .757 \mathrm{E}+00 & .310 \mathrm{E}+03 & .560 \mathrm{E}+00 & 1.45 \\ 85.602 & .753 \mathrm{E}+00 & .329 \mathrm{E}+03 & .567 \mathrm{E}+00 & 1.45 \\ 90.602 & .750 \mathrm{E}+00 & .348 \mathrm{E}+03 & .573 \mathrm{E}+00 & 1.45 \\ 95.602 & .746 \mathrm{E}+00 & .368 \mathrm{E}+03 & .579 \mathrm{E}+00 & 1.45\end{array}$


Apéndice D - Experimento 12

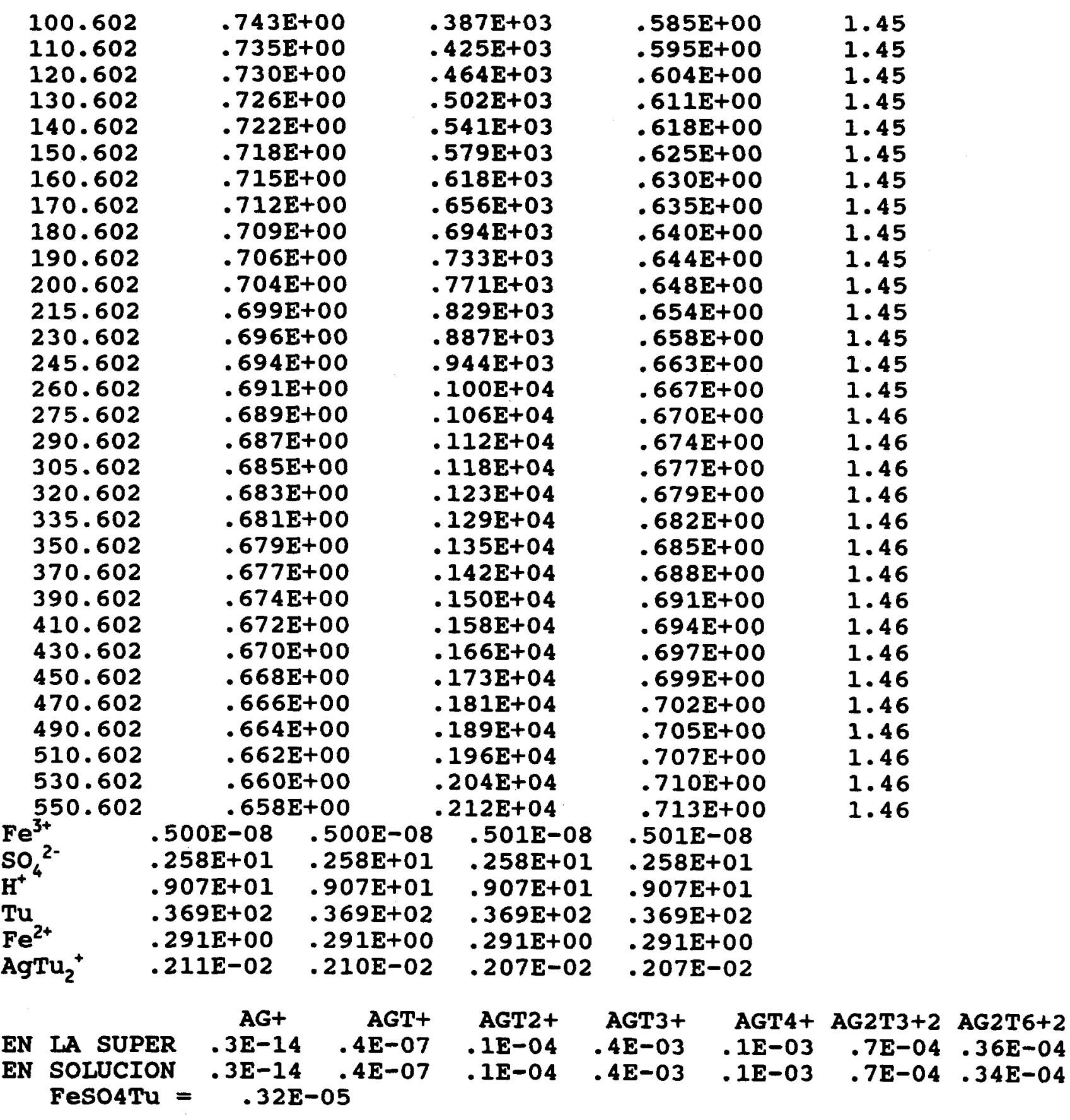

DIFUSIVIDAD EFECTIVA DE FE $+3=1.768725395291 \mathrm{E}-8$ 


\section{Apéndice D - Experimento 12}

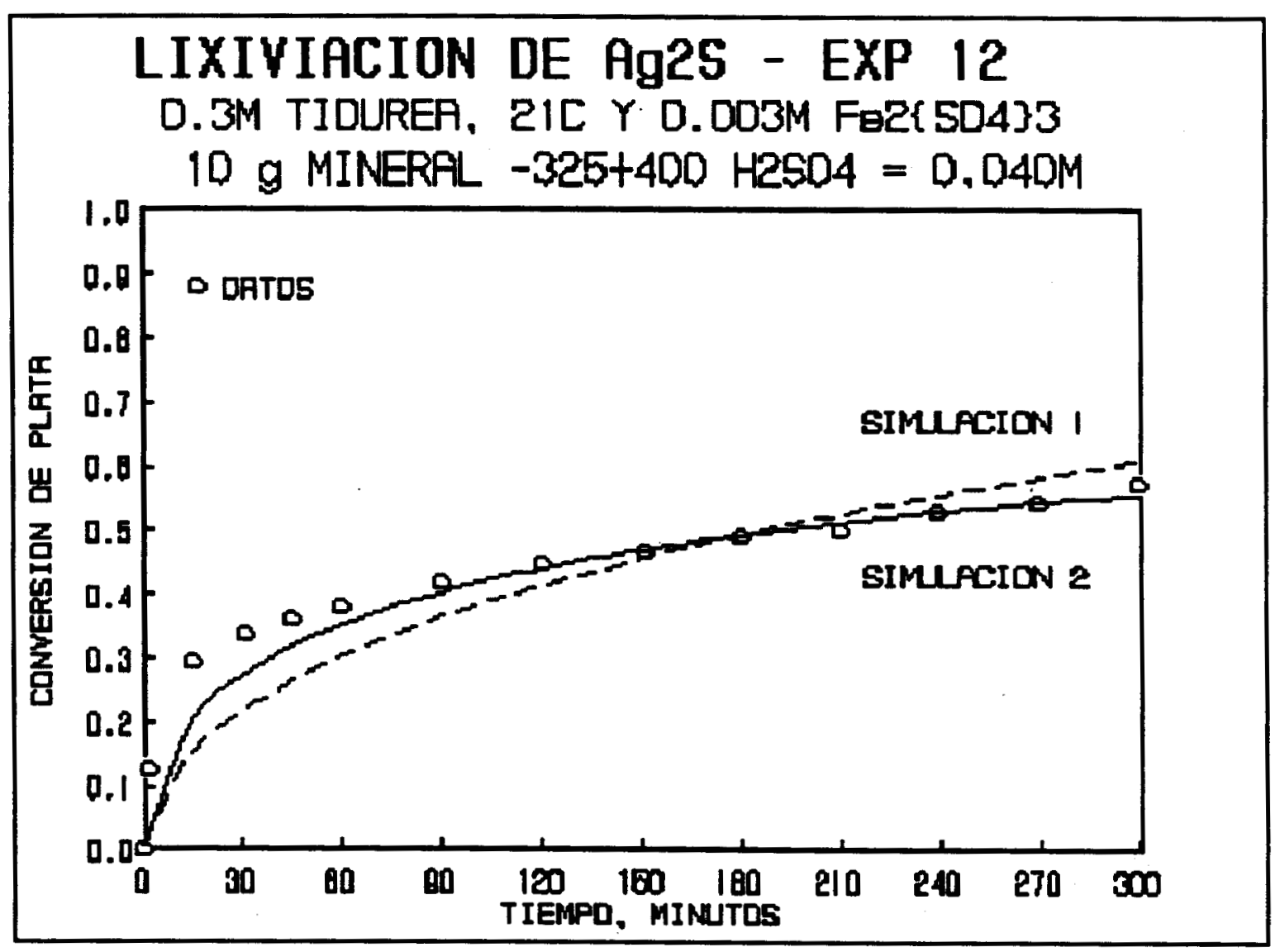




\section{EXPERIMENTO 13}

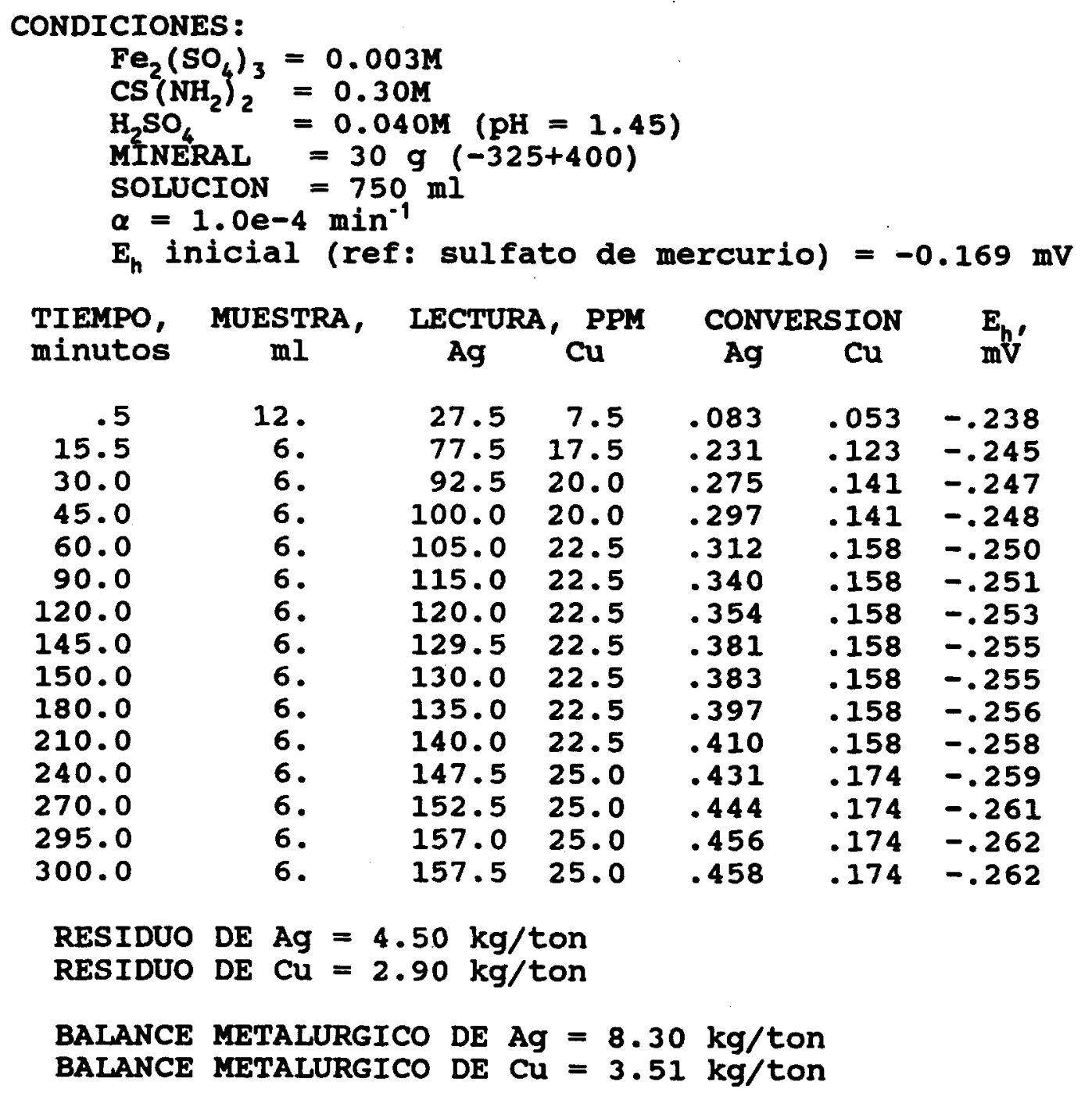


Apéndice D - Experimento 13

LIXIVIACION DE ARGENTITA

CARACTERISTICAS DEL MINERAL

GR POR TON DE AG $=.830 \mathrm{E}+04$

DENSIDAD DEL MINERAL $=\mathbf{5 . 2 0}$

RADIO DE LA PART, CM $=.202 E-02$

POROSIDAD $=0.000 \mathrm{E}+00$

PROPORCION DE SOLIDO A LIQUIDO, KG/LT $=.400 E-01$

CONSTANTES DE EQUILIBRIO A $21.00 \mathrm{C}$

CONCENTRACION INICIAL DE FE2(SO4) $3=.310 E-02$

CONCENTRACION INICIAL DE H2O2 $=0.000 \mathrm{E}+00$

CONCENTRACION INICIAL DE TIOUREA $=.300 \mathrm{E}+00$

GAMA $=.155 E-01$

VELOCIDAD DE DESCOMPOSICION DE TIOUREA = TIME* .100E-03

$\begin{array}{crcrrr}\text { FE+3 } & \text { FESO4+ } & \text { FESO4 }(\mathrm{TH})+ & \text { FEOH+2 } & \text { FE (OH) 2+ } & \text { FE2 (OH) } 2+4 \\ .447 \mathrm{E}-07 & .241 \mathrm{E}-09 & .620 \mathrm{E}-02 & .217 \mathrm{E}-08 & .145 \mathrm{E}-10 & .148 \mathrm{E}-14 \\ .0000 & .0000 & 1.0002 & .0000 & .0000 & .0000\end{array}$

FUERZA IONICA DE LA SOLUTION $=.080$

CANTIDAD DE ION FERRICO UTILIZABLE = .620E-02

DIFUSIVIDAD EFECTIVA DE FE+3 $=1.692437582451 \mathrm{E}-9$

ION FERRICO $=.266 \mathrm{E}-10$

SULFATO IIBRE $=.159 \mathrm{E}-01$

TIOUREA LIBRE $=.294 \mathrm{E}+00$

TOTAL DE ACIDO ANADIDO $=.40 \mathrm{E}-01$

FADS $=.310 \mathrm{E}-02$

OXIDANTE CONSUMIDO POR COBRE $=.384 \mathrm{E}-03$

PERFILES ADIMENSIONALES

TIEMPO DE AJUSTE $=210$.

Ag2T6+2 $=.001513577070818$ LIMITE $=.0001409949832413$

PRECIPITACION DE Ag2SO4.3Tu.H2O

DIFUSUVIDAD EFECTIVA $=4.646997286934 \mathrm{E}-10$

PH REAL $=1.448392264053$

$\begin{array}{lllll}\mathrm{Fe}^{3+} & .853 \mathrm{E}-10 & .166 \mathrm{E}-08 & .356 \mathrm{E}-08 & .413 \mathrm{E}-08 \\ \mathrm{SO}_{4}{ }^{2-} & .284 \mathrm{E}+01 & .277 \mathrm{E}+01 & .261 \mathrm{E}+01 & .257 \mathrm{E}+01 \\ \mathrm{H}^{+} & .807 \mathrm{E}+01 & .833 \mathrm{E}+01 & .901 \mathrm{E}+01 & .922 \mathrm{E}+01 \\ \mathrm{Tu}^{2+} & .467 \mathrm{E}+02 & .469 \mathrm{E}+02 & .474 \mathrm{E}+02 & .477 \mathrm{E}+02 \\ \mathrm{Fe}^{2+} & .272 \mathrm{E}+00 & .277 \mathrm{E}+00 & .288 \mathrm{E}+00 & .292 \mathrm{E}+00 \\ \mathrm{AgTu}_{2}{ }^{+} & .108 \mathrm{E}-01 & .916 \mathrm{E}-02 & .329 \mathrm{E}-02 & .184 \mathrm{E}-04\end{array}$ 
Apéndice D - Experimento 13

$\begin{array}{lcccccccc} & \text { AG+ } & \text { AGT+ } & \text { AGT2+ } & \text { AGT3+ } & \text { AGT4+ } & \text { AG2T3+2 } & \text { AG2T6+2 } \\ \text { EN IA SUPER } & .1 \mathrm{E}-13 & .2 \mathrm{E}-06 & .7 \mathrm{E}-04 & .3 \mathrm{E}-02 & .9 \mathrm{E}-03 & .2 \mathrm{E}-02 & .15 \mathrm{E}-02 \\ \text { EN SOLUCION } & .2 \mathrm{E}-16 & .3 \mathrm{E}-09 & .1 \mathrm{E}-06 & .5 \mathrm{E}-05 & .02 \mathrm{E}-05 & .4 \mathrm{E}-08 & .46 \mathrm{E}-08 \\ \text { FeSO4TU }= & .77 \mathrm{E}-07 & & & & & & \end{array}$

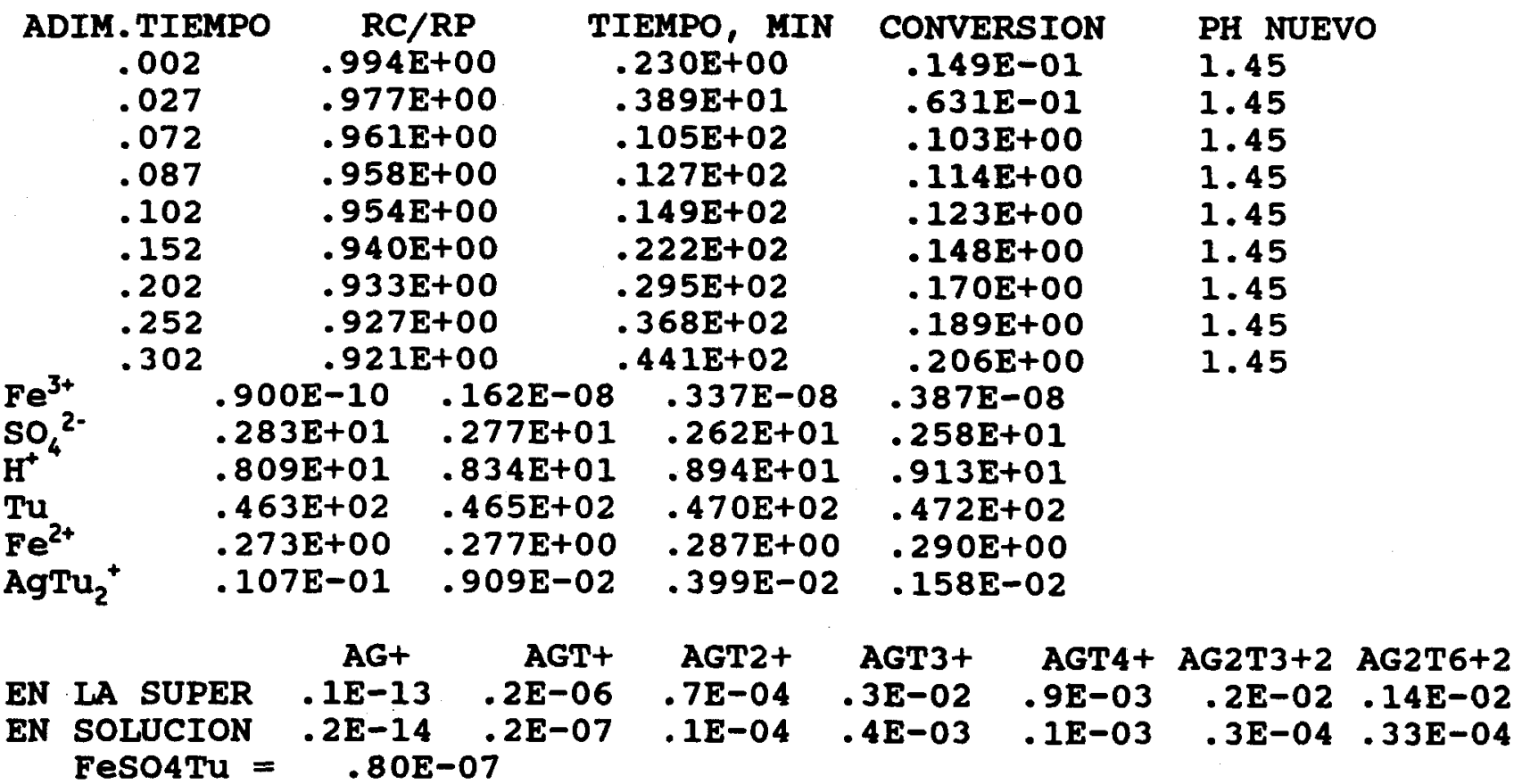

ADIM.TIEMPO
.452
.552
.652
.752
.852
1.002
1.152
1.302
1.452
1.602
1.902
2.202
2.502
2.802
3.102
3.602
4.102
4.602
5.102

RC/RP

$.901 \mathrm{E}+00$

$.892 \mathrm{E}+00$

$.884 \mathrm{E}+00$

$.877 \mathrm{E}+00$

$.870 \mathrm{E}+00$

$.857 \mathrm{E}+00$

$.848 \mathrm{E}+00$

$.839 \mathrm{E}+00$

$.831 \mathrm{E}+00$

$.823 E+00$

$.803 \mathrm{E}+00$

$.789 \mathrm{E}+00$

$.777 E+00$

$.765 \mathrm{E}+00$

$.754 \mathrm{E}+00$

$.731 \mathrm{E}+00$

$.715 \mathrm{E}+00$

$.699 E+00$

$.685 \mathrm{E}+00$

TIEMPO, MIN
$.661 \mathrm{E}+02$
$.807 \mathrm{E}+02$
$.954 \mathrm{E}+02$
$.110 \mathrm{E}+03$
$.125 \mathrm{E}+03$
$.147 \mathrm{E}+03$
$.169 \mathrm{E}+03$
$.190 \mathrm{E}+03$
$.212 \mathrm{E}+03$
$.234 \mathrm{E}+03$
$.278 \mathrm{E}+03$
$.322 \mathrm{E}+03$
$.366 \mathrm{E}+03$
$.410 \mathrm{E}+03$
$.454 \mathrm{E}+03$
$.527 \mathrm{E}+03$
$.600 \mathrm{E}+03$
$.673 \mathrm{E}+03$
$.747 \mathrm{E}+03$

CONVERSION
$.247 E+00$
$.271 E+00$
$.292 E+00$
$.311 E+00$
$.329 E+00$
$.352 E+00$
$.374 E+00$
$.394 E+00$
$.412 E+00$
$.429 E+00$
$.460 E+00$
$.487 E+00$
$.511 E+00$
$.534 E+00$
$.555 E+00$
$.586 E+00$
$.614 E+00$
$.639 E+00$
$.662 E+00$

PH NUEVO

1.45

1.45

1.45

1.45

1.45

1.46

1.46

1.46

1.46

1.46

1.46

1.46

1.46

1.46

1.46

1.46

1.46

1.46

1.46 
Apéndice D - Experimento 13

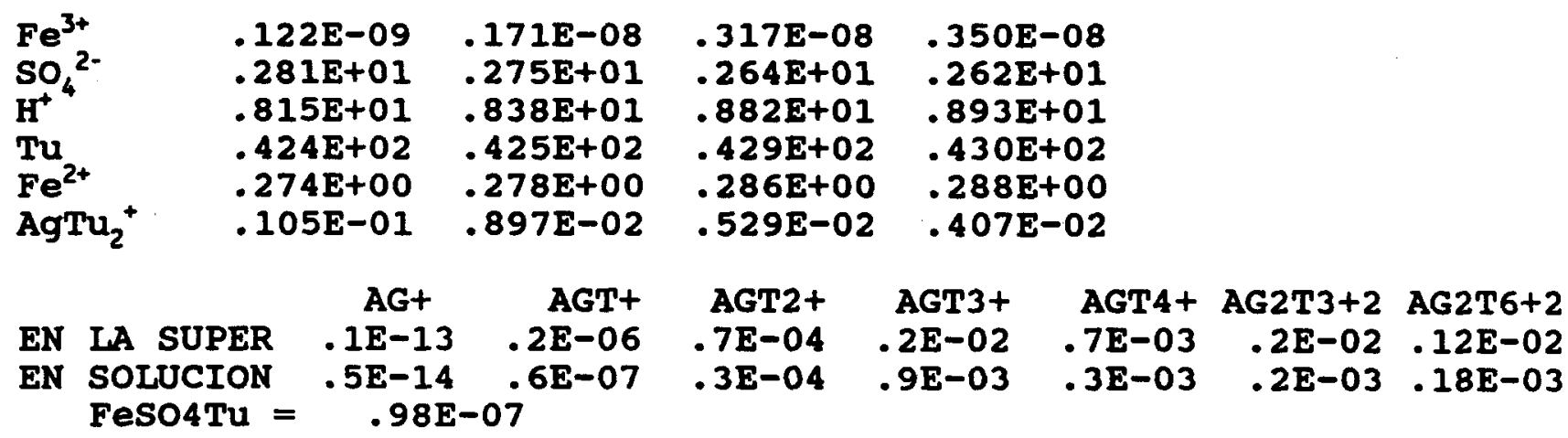

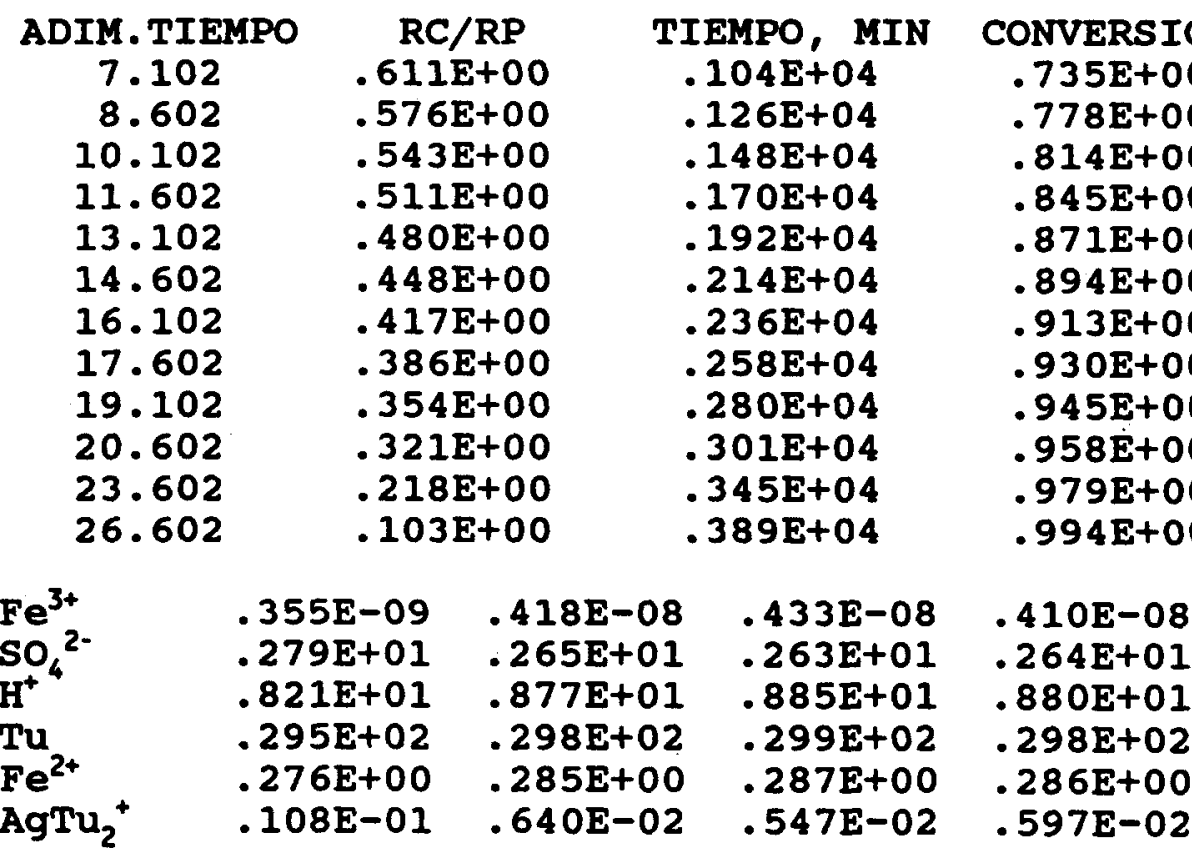

$\begin{array}{lccrrrrrr} & \text { AG+ } & \text { AGT+ } & \text { AGT2+ } & \text { AGT3+ } & \text { AGT4+ } & \text { AG2T3+2 } & \text { AG2T6+2 } \\ \text { EN IA SUPER } & .3 \mathrm{E}-13 & .2 \mathrm{E}-06 & .7 \mathrm{E}-04 & .2 \mathrm{E}-02 & .4 \mathrm{E}-03 & .3 \mathrm{E}-02 & .63 \mathrm{E}-03 \\ \text { EN SOLUCION } & .1 \mathrm{E}-13 & .1 \mathrm{E}-06 & .4 \mathrm{E}-04 & .1 \mathrm{E}-02 & .2 \mathrm{E}-03 & .7 \mathrm{E}-03 & .19 \mathrm{E}-03\end{array}$

DIFUSIVIDAD EFECTIVA DE FE+3 $=4.646997286934 \mathrm{E}-10$ CONV $=.73 \mathrm{E}-02 * t+-.27 \mathrm{E}-04 * t * \star 2+.18 \mathrm{E}-07 * t * * 3$ 
Apéndice D - Experimento 13

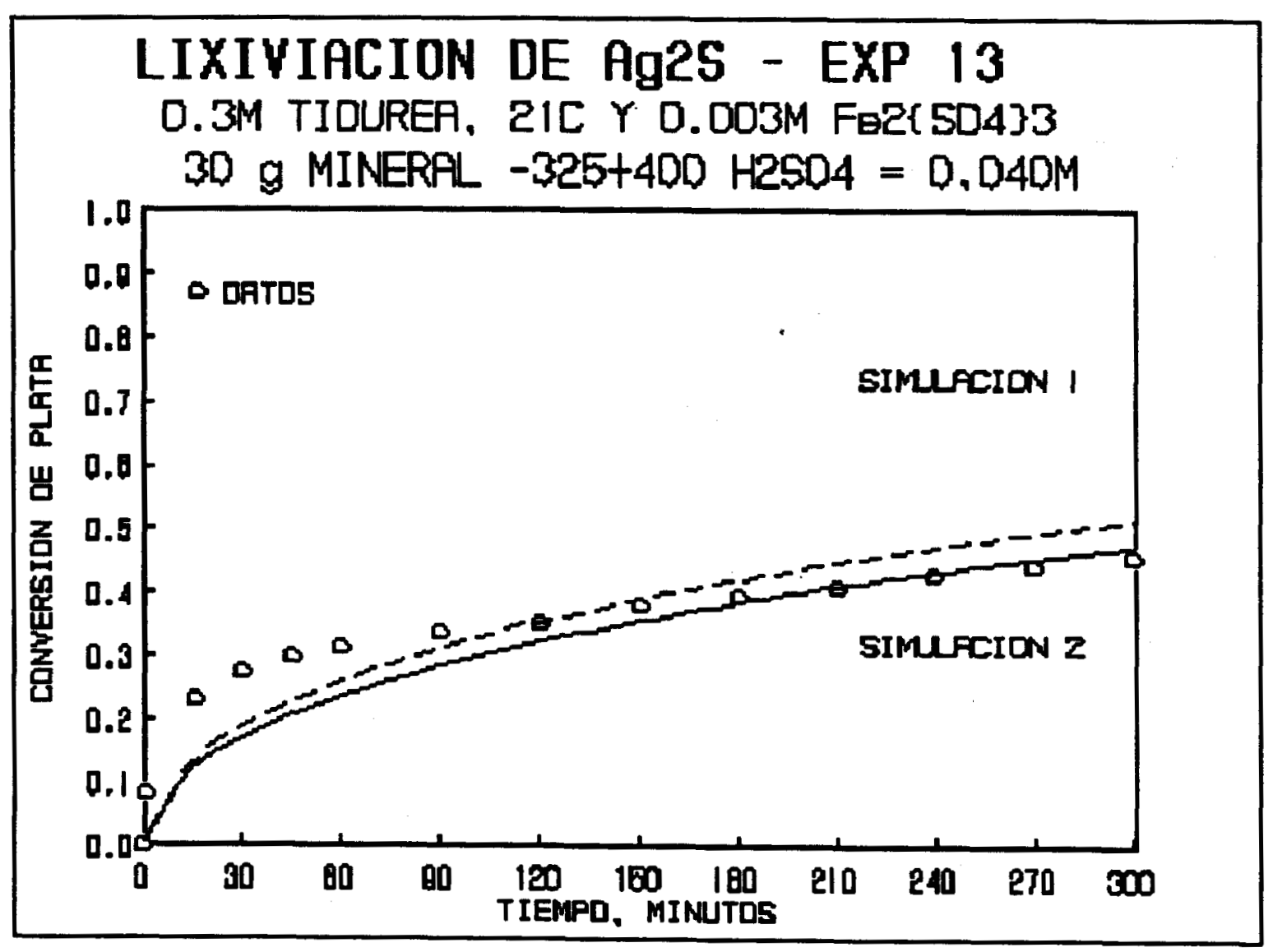




\section{Apéndice D - Experimento 14}

\section{EXPERIYENTO 14}

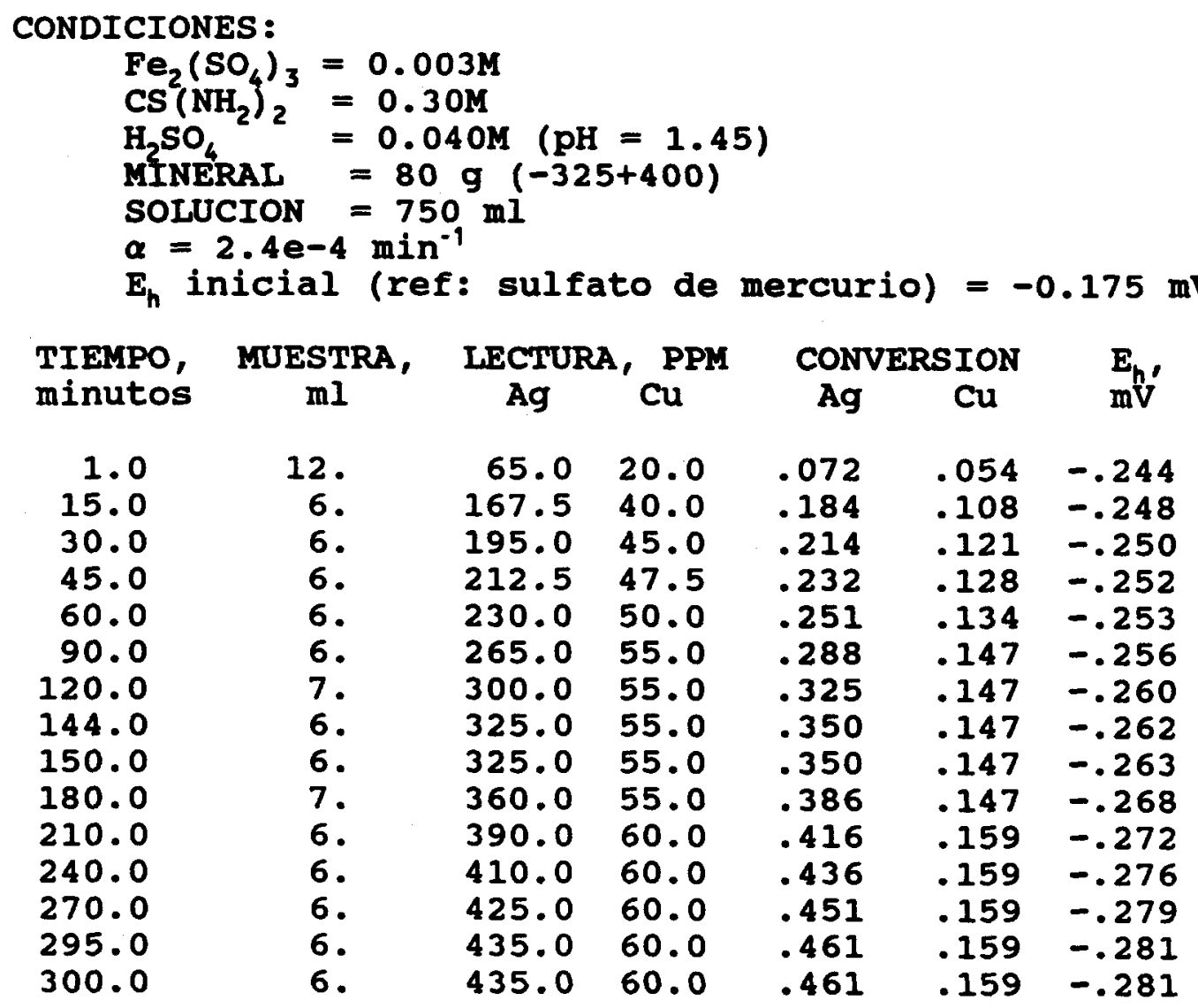

RESIDUO DE Ag $=4.56 \mathrm{~kg} /$ ton

RESIDUO DE $\mathrm{Cu}=2.90 \mathrm{~kg} /$ ton

BALANCE METALURGICO DE Ag $=8.46 \mathrm{~kg} /$ ton BALANCE METALURGICO DE $\mathrm{Cu}=3.45 \mathrm{~kg} /$ ton 


\section{Apéndice D - Experimento 14}

LIXIVIACION DE ARGENTITA

CARACTERISTICAS DEL MINERAL

GR POR TON DE AG $=.846 \mathrm{E}+04$

DENSIDAD DEL MINERAL $=5.20$

RADIO DE IA PART, CM $=.202 \mathrm{E}-02$

POROSIDAD $=0.000 E+00$

PROPORCION DE SOLIDO A LIQUIDO, KG/LT $=.107 \mathrm{E}+00$

CONSTANTES DE EQUILIBRIO A $21.00 \mathrm{C}$

CONCENTRACION INICIAL DE FE2(SO4) $3=.310 E-02$

CONCENTRACION INICIAL DE H2O2 $=0.000 \mathrm{E}+00$

CONCENTRACION INICIAL DE TIOUREA $=.300 \mathrm{E}+00$

GAMA $=.152 \mathrm{E}-01$

VELOCIDAD DE DESCOMPOSICION DE TIOUREA = TIME* .246E-03

$\begin{array}{crcrrr}\text { FE+3 } & \text { FESO4+ } & \text { FESO4 }(\mathrm{TH})+ & \text { FEOH+2 } & \text { FE (OH) 2+ } & \text { FE2 (OH) } 2+4 \\ .447 \mathrm{E}-07 & .241 \mathrm{O}-09 & .620 \mathrm{E}-02 & .217 \mathrm{E}-08 & .145 \mathrm{E}-10 & .148 \mathrm{E}-14 \\ .0000 & .0000 & 1.0002 & .0000 & .0000 & .0000\end{array}$

FUERZA IONICA DE LA SOLUTION $=.080$

CANTIDAD DE ION FERRICO UTILIZABLE $=.620 \mathrm{E}-02$

DIFUSIVIDAD EFECTIVA DE FE+3 $=1.758317109539 \mathrm{E}-9$

ION FERRICO $=.266 \mathrm{E}-10$

SULFATO LIBRE $=.159 \mathrm{E}-01$

TIOUREA LIBRE $=.294 \mathrm{E}+00$

TOTAL DE ACIDO ANADIDO $=.40 E-01$

FADS $=.310 \mathrm{E}-02$

OXIDANTE CONSUMIDO POR COBRE $=.921 \mathrm{E}-03$

PERFILES ADIMENSIONALES

TIEMPO DE AJUSTE $=45$.

Ag2T6 $+2=.001333346307812$ LIMITE $=.0001540019805052$

PRECIPITACION DE Ag2SO4.3Tu.H2O

DIFUSUVIDAD EFECTIVA $=8.256298059046 \mathrm{E}-10$

PH REAL = 1.448392264053

$\begin{array}{lllll}\mathrm{Fe}^{3+} & .968 \mathrm{E}-10 & .159 \mathrm{E}-08 & .337 \mathrm{E}-08 & .389 \mathrm{E}-08 \\ \mathrm{SO}_{4}{ }^{2-} & .281 \mathrm{E}+01 & .275 \mathrm{E}+01 & .261 \mathrm{E}+01 & .257 \mathrm{E}+01 \\ \mathrm{H}^{+} & .818 \mathrm{E}+01 & .842 \mathrm{E}+01 & .903 \mathrm{E}+01 & .922 \mathrm{E}+01 \\ \mathrm{Tu} & .472 \mathrm{E}+02 & .474 \mathrm{E}+02 & .479 \mathrm{E}+02 & .481 \mathrm{E}+02 \\ \mathrm{Fe}^{2+} & .274 \mathrm{E}+00 & .278 \mathrm{E}+00 & .289 \mathrm{E}+00 & .292 \mathrm{E}+00 \\ \mathrm{AgTu}_{2}{ }^{+} & .101 \mathrm{E}-01 & .849 \mathrm{E}-02 & .303 \mathrm{E}-02 & .466 \mathrm{E}-04\end{array}$




\section{Apéndice D - Experimento 14}

$\begin{array}{llcrrrrrr} & & \text { AGt } & \text { AGT+ } & \text { AGT2+ } & \text { AGT3+ } & \text { AGT4+ } & \text { AG2T3+2 } & \text { AG2T6+2 } \\ \text { EN LA SUPER } & .1 \mathrm{E}-13 & .1 \mathrm{E}-06 & .6 \mathrm{E}-04 & .3 \mathrm{E}-02 & .9 \mathrm{E}-03 & .1 \mathrm{E}-02 & .13 \mathrm{E}-02 \\ \text { EN SOLUCION } & .4 \mathrm{E}-16 & .6 \mathrm{E}-09 & .3 \mathrm{E}-06 & .1 \mathrm{E}-04 & .4 \mathrm{E}-05 & .3 \mathrm{E}-07 & .30 \mathrm{E}-07\end{array}$

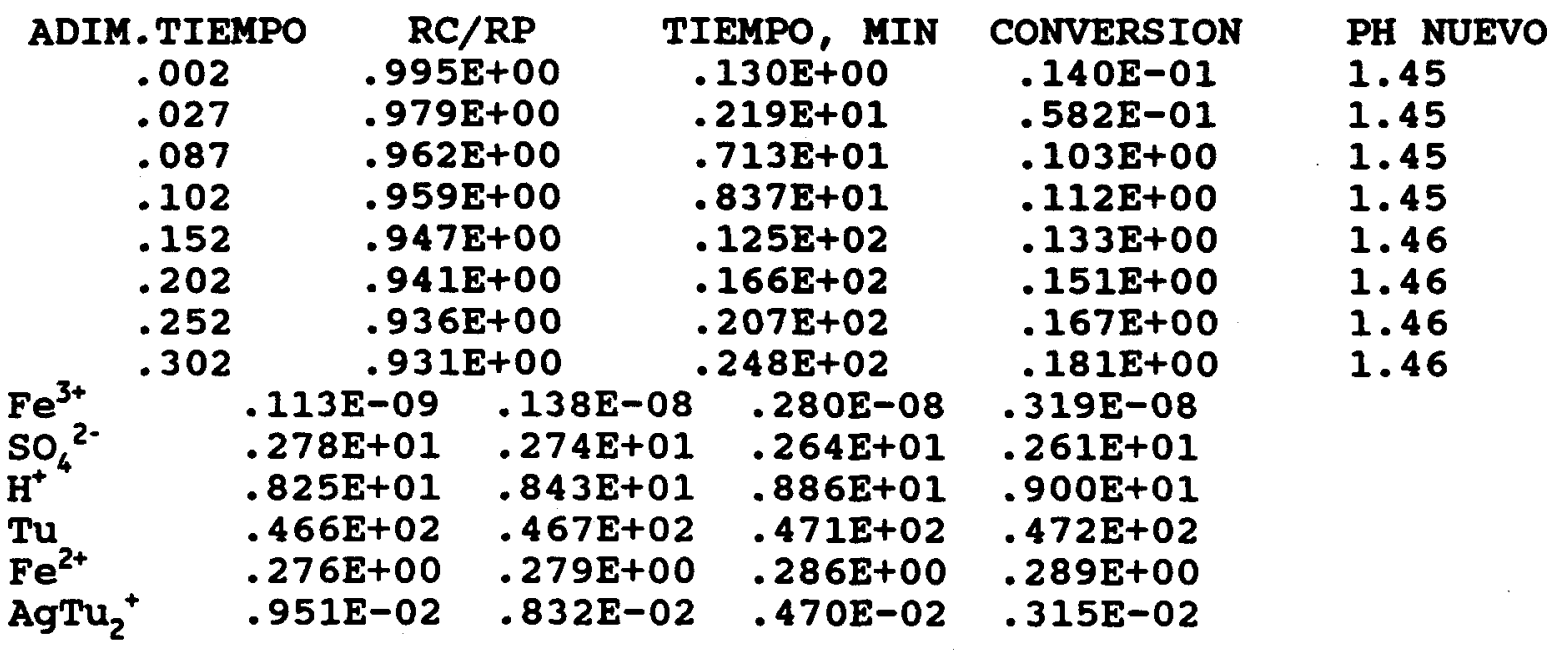

$\begin{array}{llcrrrrrr} & & \text { AGt } & \text { AGTt } & \text { AGT2+ } & \text { AGT3+ } & \text { AGT4+ } & \text { AG2T3+2 } & \text { AG2T6+2 } \\ \text { EN IA SUPER } & .1 \mathrm{E}-13 & .1 \mathrm{E}-06 & .6 \mathrm{E}-04 & .2 \mathrm{E}-02 & .8 \mathrm{E}-03 & .1 \mathrm{E}-02 & .12 \mathrm{E}-02 \\ \text { EN SOLUCION } & .3 \mathrm{E}-14 & .4 \mathrm{E}-07 & .2 \mathrm{E}-04 & .8 \mathrm{E}-03 & .3 \mathrm{E}-03 & .1 \mathrm{E}-03 & .13 \mathrm{E}-03\end{array}$

$\begin{array}{ccccl}\text { ADIM. TIEMPO } & \text { RC/RP } & \text { TIEMPO, MIN } & \text { CONVERSION } & \text { PH NUEVO } \\ .452 & .916 E+00 & .372 E+02 & .215 E+00 & 1.46 \\ .552 & .910 E+00 & .454 E+02 & .233 E+00 & 1.46 \\ .652 & .904 E+00 & .537 E+02 & .249 E+00 & 1.46 \\ .752 & .898 E+00 & .619 E+02 & .264 E+00 & 1.46 \\ .852 & .893 E+00 & .701 E+02 & .277 E+00 & 1.46 \\ 1.002 & .884 E+00 & .825 E+02 & .294 E+00 & 1.46 \\ 1.152 & .878 E+00 & .949 E+02 & .310 E+00 & 1.47 \\ 1.302 & .873 E+00 & .107 E+03 & .324 E+00 & 1.47 \\ 1.452 & .868 E+00 & .120 E+03 & .337 E+00 & 1.47 \\ 1.602 & .863 E+00 & .132 E+03 & .348 E+00 & 1.47 \\ 1.852 & .853 E+00 & .153 E+03 & .366 E+00 & 1.47 \\ 2.102 & .847 E+00 & .173 E+03 & .381 E+00 & 1.47 \\ 2.352 & .841 E+00 & .194 E+03 & .395 E+00 & 1.47 \\ 2.602 & .835 E+00 & .214 E+03 & .407 E+00 & 1.47 \\ 2.852 & .830 E+00 & .235 E+03 & .418 E+00 & 1.47 \\ 3.352 & .818 E+00 & .276 E+03 & .438 E+00 & 1.47 \\ 3.852 & .811 E+00 & .317 E+03 & .454 E+00 & 1.47 \\ 4.352 & .804 E+00 & .358 E+03 & .469 E+00 & 1.47 \\ 4.852 & .798 E+00 & .400 E+03 & .482 E+00 & 1.47\end{array}$


Apéndice D - Experimento 14

$\begin{array}{lllll}\mathrm{Fe}^{3+} & .204 \mathrm{E}-09 & .954 \mathrm{E}-09 & .174 \mathrm{E}-08 & .192 \mathrm{E}-08 \\ \mathrm{SO}_{4}{ }^{2-} & .273 \mathrm{E}+01 & .271 \mathrm{E}+01 & .268 \mathrm{E}+01 & .267 \mathrm{E}+01 \\ \mathrm{H}^{+} & .842 \mathrm{E}+01 & .848 \mathrm{E}+01 & .862 \mathrm{E}+01 & .866 \mathrm{E}+01 \\ \mathrm{Tu}^{2+} & .415 \mathrm{E}+02 & .415 \mathrm{E}+02 & .416 \mathrm{E}+02 & .416 \mathrm{E}+02 \\ \mathrm{Fe}^{2+} & .280 \mathrm{E}+00 & .281 \mathrm{E}+00 & .283 \mathrm{E}+00 & .284 \mathrm{E}+00 \\ \mathrm{AgTu}_{2}{ }^{+} & .854 \mathrm{E}-02 & .807 \mathrm{E}-02 & .693 \mathrm{E}-02 & .657 \mathrm{E}-02\end{array}$

$\begin{array}{lcccccccc} & \text { AGt } & \text { AGT+ } & \text { AGT2+ } & \text { AGT3+ } & \text { AGT4+ } & \text { AG2T3+2 } & \text { AG2T6+2 } \\ \text { EN LA SUPER } & .1 \mathrm{E}-13 & .1 \mathrm{E}-06 & .5 \mathrm{E}-04 & .2 \mathrm{E}-02 & .6 \mathrm{E}-03 & .1 \mathrm{E}-02 & .74 \mathrm{E}-03 \\ \text { EN SOLUCION } & .8 \mathrm{E}-14 & .1 \mathrm{E}-06 & .4 \mathrm{E}-04 & .1 \mathrm{E}-02 & .4 \mathrm{E}-03 & .6 \mathrm{E}-03 & .44 \mathrm{E}-03 \\ \text { FeSO4TU }= & .16 \mathrm{E}-06 & & & & & & \end{array}$

$\begin{array}{ccccl}\text { ADIM.TIEMPO } & \text { RC/RP } & \text { TIEMPO, MIN } & \text { CONVERSION } & \text { PH NUEVO } \\ 6.852 & .773 E+00 & .564 E+03 & .519 E+00 & 1.48 \\ 8.352 & .764 E+00 & .688 E+03 & .538 E+00 & 1.48 \\ 9.852 & .757 E+00 & .811 E+03 & .554 E+00 & 1.48 \\ 11.352 & .752 E+00 & .935 E+03 & .566 E+00 & 1.48 \\ 12.852 & .747 E+00 & .106 E+04 & .576 E+00 & 1.48 \\ 14.352 & .743 E+00 & .118 E+04 & .584 E+00 & 1.48 \\ 15.852 & .740 E+00 & .131 E+04 & .590 E+00 & 1.48 \\ 17.352 & .737 E+00 & .143 E+04 & .596 E+00 & 1.48 \\ 18.852 & .735 E+00 & .155 E+04 & .600 E+00 & 1.48 \\ 20.352 & .733 E+00 & .168 E+04 & .604 E+00 & 1.48 \\ 23.352 & .730 E+00 & .192 E+04 & .608 E+00 & 1.48 \\ 26.352 & .729 E+00 & .217 E+04 & .610 E+00 & 1.48 \\ 29.352 & .730 E+00 & .242 E+04 & .611 E+00 & 1.48 \\ 32.352 & .732 E+00 & .266 E+04 & .609 E+00 & 1.48 \\ 35.352 & .735 E+00 & .291 E+04 & .606 E+00 & 1.48 \\ 38.352 & .742 E+00 & .316 E+04 & .598 E+00 & 1.48 \\ 41.352 & .757 E+00 & .341 E+04 & .581 E+00 & 1.48\end{array}$


Apéndice D - Experimento 14

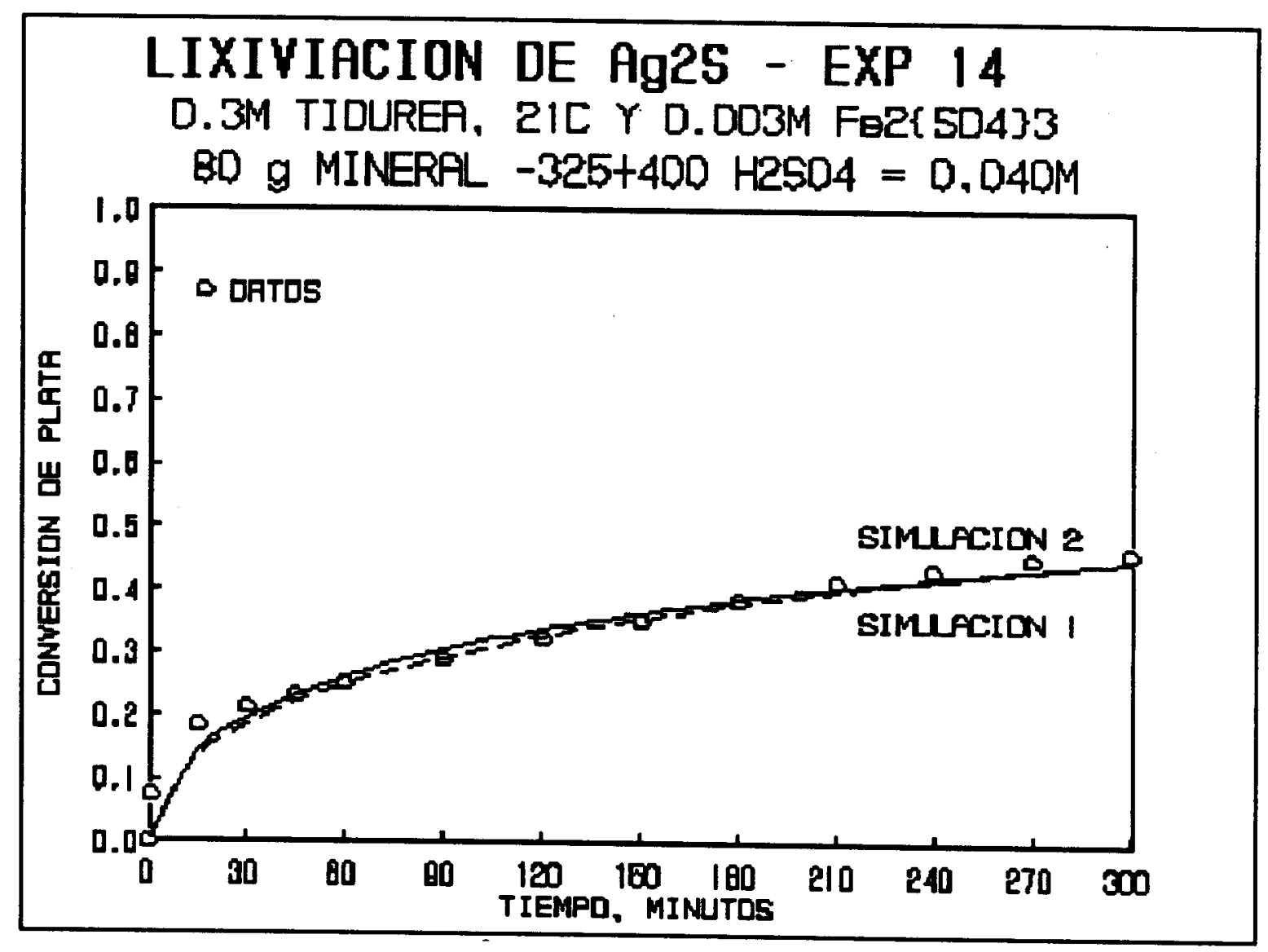




\section{EXPERIMENTO 15}

\section{CONDICIONES :}

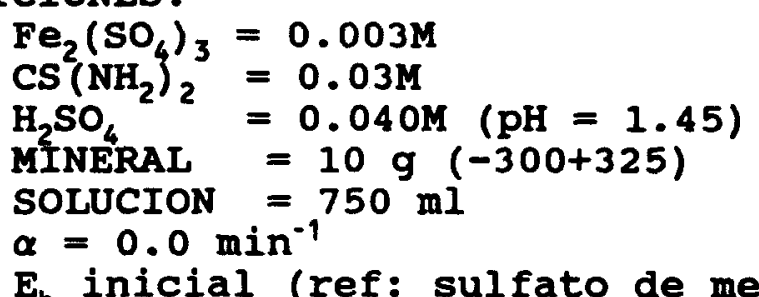

$\mathrm{E}_{\mathrm{h}}$ inicial (ref: sulfato de mercurio) $=-0.098 \mathrm{mV}$

\begin{tabular}{rcccccc} 
TIEMPO, & MUESTRA, & \multicolumn{2}{c}{ LECTURA, PPM } & \multicolumn{2}{c}{ CONVERSION } & $E_{h \prime}$ \\
minutos & ml & Ag & Cu & Ag & Cu & mV \\
1.5 & 12. & 4.0 & 1.6 & .039 & .037 & -.126 \\
15.0 & 6. & 11.0 & 2.6 & .105 & .059 & -.180 \\
31.0 & 6. & 15.0 & 3.2 & .143 & .073 & -.180 \\
45.5 & 6. & 18.5 & 3.6 & .175 & .081 & -.180 \\
60.5 & 6. & 21.5 & 4.0 & .203 & .090 & -.181 \\
90.0 & 6. & 27.0 & 4.6 & .253 & .103 & -.181 \\
120.0 & 6. & 31.8 & 5.1 & .297 & .114 & -.181 \\
146.0 & 6. & 36.6 & 5.5 & .340 & .123 & -.182 \\
151.0 & 6. & 36.6 & 5.5 & .340 & .123 & -.182 \\
180.0 & 6. & 39.6 & 5.8 & .367 & .129 & -.183 \\
210.0 & 6. & 46.2 & 6.0 & .425 & .133 & -.184 \\
240.0 & 6. & 48.0 & 6.2 & .441 & .137 & -.185 \\
270.0 & 6. & 51.6 & 6.4 & .472 & .141 & -.185 \\
296.0 & 6. & 53.4 & 6.6 & .487 & .145 & -.185 \\
301.0 & 6. & 53.4 & 6.6 & .487 & .145 & -.185
\end{tabular}

RESIDUO DE Ag $=3.99 \mathrm{~kg} / \mathrm{ton}$

RESIDUO DE Cu $=2.80 \mathrm{~kg} /$ ton

BALANCE METALURGICO DE Ag $=7.78 \mathrm{~kg} / \mathrm{ton}$

BALANCE METALURGICO DE $\mathrm{Cu}=3.28 \mathrm{~kg} / \mathrm{ton}$ 
Apéndice D - Experimento 15

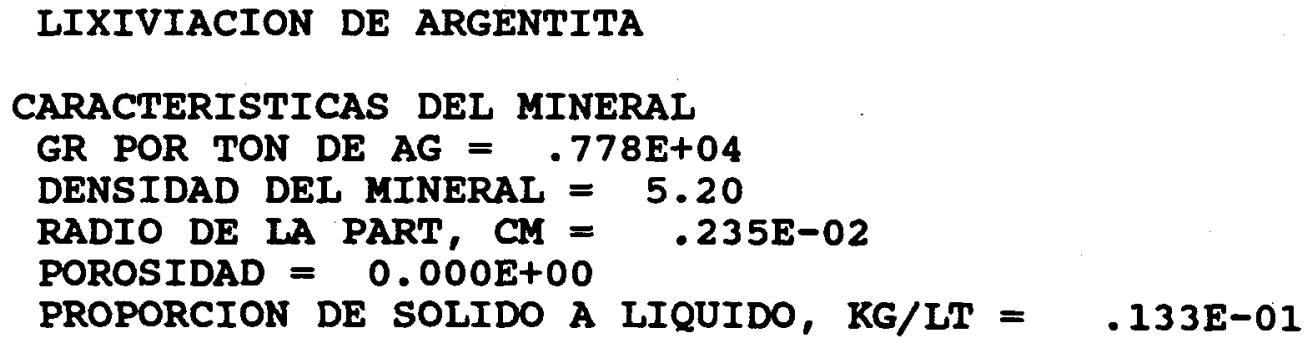

PERFILES ADIMENSIONALES

TIEMPO DE AJUSTE $=151$.

DIFUSUVIDAD EFECTIVA $=4.628986969233 \mathrm{E}-10$

PH REAL $=1.450476158382$

$\begin{array}{lllll}\mathrm{Fe}^{3+} & .856 \mathrm{E}-09 & .198 \mathrm{E}-07 & .437 \mathrm{E}-07 & .513 \mathrm{E}-07 \\ \mathrm{SO}_{4}{ }^{2-} & .296 \mathrm{E}+01 & .287 \mathrm{E}+01 & .264 \mathrm{E}+01 & .256 \mathrm{E}+01 \\ \mathrm{H}^{+} & .755 \mathrm{E}+01 & .789 \mathrm{E}+01 & .883 \mathrm{E}+01 & .917 \mathrm{E}+01 \\ \mathrm{Tu}^{2+} & .365 \mathrm{E}+01 & .370 \mathrm{E}+01 & .386 \mathrm{E}+01 & .392 \mathrm{E}+01 \\ \mathrm{Fe}^{2+} & .264 \mathrm{E}+00 & .270 \mathrm{E}+00 & .286 \mathrm{E}+00 & .292 \mathrm{E}+00 \\ \mathrm{AgTu}_{2}{ }^{+} & .640 \mathrm{E}-02 & .571 \mathrm{E}-02 & .298 \mathrm{E}-02 & .632 \mathrm{E}-04\end{array}$




\section{Apéndice $D$ - Experimento 15}

$\begin{array}{llcrrrrrr} & \text { AG+ } & \text { AGT+ } & \text { AGT2+ } & \text { AGT3+ } & \text { AGT4+ } & \text { AG2T3+2 } & \text { AG2T6+2 } \\ \text { EN LA SUPER } & .1 \mathrm{E}-11 & .1 \mathrm{E}-05 & .4 \mathrm{E}-04 & .1 \mathrm{E}-03 & .3 \mathrm{E}-05 & .7 \mathrm{E}-02 & .32 \mathrm{E}-05 \\ \text { EN SOLUCION } & .9 \mathrm{E}-14 & .1 \mathrm{E}-07 & .4 \mathrm{E}-06 & .1 \mathrm{E}-05 & .4 \mathrm{E}-07 & .6 \mathrm{E}-06 & .36 \mathrm{E}-09\end{array}$

$\begin{array}{cc}\text { ADIM. TIEMPO } & \text { RC/RP } \\ .003 & .991 \mathrm{E}+00 \\ .053 & .965 \mathrm{E}+00 \\ .083 & .952 \mathrm{E}+00 \\ .113 & .946 \mathrm{E}+00 \\ .143 & .940 \mathrm{E}+00 \\ .173 & .935 \mathrm{E}+00 \\ .203 & .930 \mathrm{E}+00 \\ .303 & .908 \mathrm{E}+00 \\ .403 & .897 \mathrm{E}+00 \\ .503 & .887 \mathrm{E}+00 \\ .603 & .877 \mathrm{E}+00\end{array}$

TIEMPO, MIN CONVERSION

$.636 \mathrm{E}+00$

$.106 \mathrm{E}+02$

$.165 \mathrm{E}+02$

$.225 E+02$

$.285 \mathrm{E}+02$

$.344 \mathrm{E}+02$

$.404 \mathrm{E}+02$

$.603 \mathrm{E}+02$

$.802 \mathrm{E}+02$

$.100 \mathrm{E}+03$

$.120 \mathrm{E}+03$
$.227 \mathrm{E}-01$

$.944 \mathrm{E}-01$

$.118 \mathrm{E}+00$

$.137 \mathrm{E}+00$

$.155 \mathrm{E}+00$

$.170 \mathrm{E}+00$

$.184 \mathrm{E}+00$

$.222 \mathrm{E}+00$

$.254 E+00$

$.282 \mathrm{E}+00$

$.306 E+00$

$\begin{array}{lllll}\mathrm{Fe}^{3+} & .852 \mathrm{E}-09 & .206 \mathrm{E}-07 & .434 \mathrm{E}-07 & .500 \mathrm{E}-07 \\ \mathrm{SO}_{4}{ }^{2-} & .295 \mathrm{E}+01 & .285 \mathrm{E}+01 & .263 \mathrm{E}+01 & .257 \mathrm{E}+01 \\ \mathrm{H}^{+} & .759 \mathrm{E}+01 & .794 \mathrm{E}+01 & .883 \mathrm{E}+01 & .913 \mathrm{E}+01 \\ \mathrm{Tu}^{2+} & .364 \mathrm{E}+01 & .369 \mathrm{E}+01 & .384 \mathrm{E}+01 & .389 \mathrm{E}+01 \\ \mathrm{Fe}^{2+} & .265 \mathrm{E}+00 & .271 \mathrm{E}+00 & .287 \mathrm{E}+00 & .292 \mathrm{E}+00 \\ \mathrm{AgTu}_{2}{ }^{+} \cdot & .630 \mathrm{E}-02 & .556 \mathrm{E}-02 & .292 \mathrm{E}-02 & .952 \mathrm{E}-03\end{array}$

$\begin{array}{lllll}\mathrm{Fe}^{3+} & .852 \mathrm{E}-09 & .206 \mathrm{E}-07 & .434 \mathrm{E}-07 & .500 \mathrm{E}-07 \\ \mathrm{SO}_{4}{ }^{2-} & .295 \mathrm{E}+01 & .285 \mathrm{E}+01 & .263 \mathrm{E}+01 & .257 \mathrm{E}+01 \\ \mathrm{H}^{+} & .759 \mathrm{E}+01 & .794 \mathrm{E}+01 & .883 \mathrm{E}+01 & .913 \mathrm{E}+01 \\ \mathrm{Tu}^{2+} & .364 \mathrm{E}+01 & .369 \mathrm{E}+01 & .384 \mathrm{E}+01 & .389 \mathrm{E}+01 \\ \mathrm{Fe}^{2+} & .265 \mathrm{E}+00 & .271 \mathrm{E}+00 & .287 \mathrm{E}+00 & .292 \mathrm{E}+00 \\ \mathrm{AgTu}_{2}{ }^{+} \cdot & .630 \mathrm{E}-02 & .556 \mathrm{E}-02 & .292 \mathrm{E}-02 & .952 \mathrm{E}-03\end{array}$

$\begin{array}{lllll}\mathrm{Fe}^{3+} & .852 \mathrm{E}-09 & .206 \mathrm{E}-07 & .434 \mathrm{E}-07 & .500 \mathrm{E}-07 \\ \mathrm{SO}_{4}{ }^{2-} & .295 \mathrm{E}+01 & .285 \mathrm{E}+01 & .263 \mathrm{E}+01 & .257 \mathrm{E}+01 \\ \mathrm{H}^{+} & .759 \mathrm{E}+01 & .794 \mathrm{E}+01 & .883 \mathrm{E}+01 & .913 \mathrm{E}+01 \\ \mathrm{Tu}^{2+} & .364 \mathrm{E}+01 & .369 \mathrm{E}+01 & .384 \mathrm{E}+01 & .389 \mathrm{E}+01 \\ \mathrm{Fe}^{2+} & .265 \mathrm{E}+00 & .271 \mathrm{E}+00 & .287 \mathrm{E}+00 & .292 \mathrm{E}+00 \\ \mathrm{AgTu}_{2}{ }^{+} \cdot & .630 \mathrm{E}-02 & .556 \mathrm{E}-02 & .292 \mathrm{E}-02 & .952 \mathrm{E}-03\end{array}$

$\begin{array}{lllll}\mathrm{Fe}^{3+} & .852 \mathrm{E}-09 & .206 \mathrm{E}-07 & .434 \mathrm{E}-07 & .500 \mathrm{E}-07 \\ \mathrm{SO}_{4}{ }^{2-} & .295 \mathrm{E}+01 & .285 \mathrm{E}+01 & .263 \mathrm{E}+01 & .257 \mathrm{E}+01 \\ \mathrm{H}^{+} & .759 \mathrm{E}+01 & .794 \mathrm{E}+01 & .883 \mathrm{E}+01 & .913 \mathrm{E}+01 \\ \mathrm{Tu}^{2+} & .364 \mathrm{E}+01 & .369 \mathrm{E}+01 & .384 \mathrm{E}+01 & .389 \mathrm{E}+01 \\ \mathrm{Fe}^{2+} & .265 \mathrm{E}+00 & .271 \mathrm{E}+00 & .287 \mathrm{E}+00 & .292 \mathrm{E}+00 \\ \mathrm{AgTu}_{2}{ }^{+} \cdot & .630 \mathrm{E}-02 & .556 \mathrm{E}-02 & .292 \mathrm{E}-02 & .952 \mathrm{E}-03\end{array}$

$\begin{array}{lllll}\mathrm{Fe}^{3+} & .852 \mathrm{E}-09 & .206 \mathrm{E}-07 & .434 \mathrm{E}-07 & .500 \mathrm{E}-07 \\ \mathrm{SO}_{4}{ }^{2-} & .295 \mathrm{E}+01 & .285 \mathrm{E}+01 & .263 \mathrm{E}+01 & .257 \mathrm{E}+01 \\ \mathrm{H}^{+} & .759 \mathrm{E}+01 & .794 \mathrm{E}+01 & .883 \mathrm{E}+01 & .913 \mathrm{E}+01 \\ \mathrm{Tu}^{2+} & .364 \mathrm{E}+01 & .369 \mathrm{E}+01 & .384 \mathrm{E}+01 & .389 \mathrm{E}+01 \\ \mathrm{Fe}^{2+} & .265 \mathrm{E}+00 & .271 \mathrm{E}+00 & .287 \mathrm{E}+00 & .292 \mathrm{E}+00 \\ \mathrm{AgTu}_{2}{ }^{+} \cdot & .630 \mathrm{E}-02 & .556 \mathrm{E}-02 & .292 \mathrm{E}-02 & .952 \mathrm{E}-03\end{array}$

$\begin{array}{lllll}\mathrm{Fe}^{3+} & .852 \mathrm{E}-09 & .206 \mathrm{E}-07 & .434 \mathrm{E}-07 & .500 \mathrm{E}-07 \\ \mathrm{SO}_{4}{ }^{2-} & .295 \mathrm{E}+01 & .285 \mathrm{E}+01 & .263 \mathrm{E}+01 & .257 \mathrm{E}+01 \\ \mathrm{H}^{+} & .759 \mathrm{E}+01 & .794 \mathrm{E}+01 & .883 \mathrm{E}+01 & .913 \mathrm{E}+01 \\ \mathrm{Tu}^{2+} & .364 \mathrm{E}+01 & .369 \mathrm{E}+01 & .384 \mathrm{E}+01 & .389 \mathrm{E}+01 \\ \mathrm{Fe}^{2+} & .265 \mathrm{E}+00 & .271 \mathrm{E}+00 & .287 \mathrm{E}+00 & .292 \mathrm{E}+00 \\ \mathrm{AgTu}_{2}{ }^{+} \cdot & .630 \mathrm{E}-02 & .556 \mathrm{E}-02 & .292 \mathrm{E}-02 & .952 \mathrm{E}-03\end{array}$

$\begin{array}{llcrrrrrr} & & \text { AG+ } & \text { AGT+ } & \text { AGT2+ } & \text { AGT3+ } & \text { AGT4+ } & \text { AG2T3+2 } & \text { AG2T6+2 } \\ \text { EN LA SUPER } & .1 \mathrm{E}-11 & .1 \mathrm{E}-05 & .4 \mathrm{E}-04 & .1 \mathrm{E}-03 & .3 \mathrm{E}-05 & .7 \mathrm{E}-02 & .31 \mathrm{E}-05 \\ \text { EN SOLUCION } & .1 \mathrm{E}-12 & .2 \mathrm{E}-06 & .6 \mathrm{E}-05 & .2 \mathrm{E}-04 & .6 \mathrm{E}-06 & .1 \mathrm{E}-03 & .81 \mathrm{E}-07\end{array}$

TIEMPO, MIN

$.180 \mathrm{E}+03$

$.219 E+03$

$.259 \mathrm{E}+03$

$.299 \mathrm{E}+03$

$.339 E+03$

$.398 \mathrm{E}+03$

$.458 \mathrm{E}+03$

$.518 \mathrm{E}+03$

$.577 \mathrm{E}+03$

$.637 \mathrm{E}+03$

$.736 \mathrm{E}+03$

$.836 \mathrm{E}+03$

$.935 \mathrm{E}+03$

$.103 E+04$

$.113 E+04$

$.133 E+04$

$.153 \mathrm{E}+04$

\section{CONVERSION}

. 368E+00

$.402 E+00$

$.433 \mathrm{E}+00$

$.461 \mathrm{E}+00$

$.486 \mathrm{E}+00$

$.520 \mathrm{E}+00$

$.551 \mathrm{E}+00$

$.579 E+00$

$.604 E+00$

$.628 \mathrm{E}+00$

$.664 \mathrm{E}+00$

$.695 E+00$

$.724 \mathrm{E}+00$

$.750 \mathrm{E}+00$

$.774 \mathrm{E}+00$

$.815 \mathrm{E}+00$

$.851 \mathrm{E}+00$
PH NUEVO

1.45

1.45

1.45

1.45

1.45

1.45

1.45

1.45

1.45

1.45

1.45

6.703

7.703

$.499 \mathrm{E}+00$

PH NUEVO

1.45

1.45

1.45

1.45

1.45

1.45

1.45

1.45

1.45

1.45

1.45

1.45

1.45

1.46

1.46

1.46

1.46 


\section{Apéndice D - Experimento 15}

$\begin{array}{lccccc} & 8.703 & .461 \mathrm{E}+00 & .173 \mathrm{E}+04 & .881 \mathrm{E}+00 & 1.46 \\ \mathrm{Fe}^{3+} & 9.703 & .422 \mathrm{E}+00 & .193 \mathrm{E}+04 & .907 \mathrm{E}+00 & 1.46 \\ \mathrm{SO}_{4}{ }^{2-} & .841 \mathrm{E}-09 & .275 \mathrm{E}-07 & .448 \mathrm{E}-07 & .475 \mathrm{E}-07 & \\ \mathrm{H}^{+} & .293 \mathrm{E}+01 & .277 \mathrm{E}+01 & .261 \mathrm{E}+01 & .258 \mathrm{E}+01 \\ \mathrm{Tu}^{2+} & .766 \mathrm{E}+01 & .824 \mathrm{E}+01 & .893 \mathrm{E}+01 & .905 \mathrm{E}+01 \\ \mathrm{Fe}^{2+} & .361 \mathrm{E}+01 & .370 \mathrm{E}+01 & .382 \mathrm{E}+01 & .384 \mathrm{E}+01 \\ \mathrm{AgTu}_{2}{ }^{+} & .266 \mathrm{E}+00 & .276 \mathrm{E}+00 & .289 \mathrm{E}+00 & .291 \mathrm{E}+00 \\ \end{array}$

$\begin{array}{llcrrrrrr} & & \text { AGt } & \text { AGT+ } & \text { AGT2+ } & \text { AGT3+ } & \text { AGT4+ } & \text { AG2T3+2 } & \text { AG2T6+2 } \\ \text { EN LA SUPER } & .1 \mathrm{E}-11 & .1 \mathrm{E}-05 & .4 \mathrm{E}-04 & .1 \mathrm{E}-03 & .3 \mathrm{E}-05 & .6 \mathrm{E}-02 & .29 \mathrm{E}-05 \\ \text { EN SOLUCION } & .2 \mathrm{E}-12 & .3 \mathrm{E}-06 & .1 \mathrm{E}-04 & .3 \mathrm{E}-04 & .9 \mathrm{E}-06 & .4 \mathrm{E}-03 & .23 \mathrm{E}-06\end{array}$

$\begin{array}{ccccc}\text { ADIM.TIEMPO } & \text { RC/RP } & \text { TIEMPO, MIN } & \text { CONVERSION } & \text { PH NUEVO } \\ 13.703 & .155 E+00 & .272 E+04 & .980 E+00 & 1.46\end{array}$

$\begin{array}{lllll}\mathrm{Fe}^{3+} & .836 \mathrm{E}-09 & .387 \mathrm{E}-07 & .465 \mathrm{E}-07 & .454 \mathrm{E}-07 \\ \mathrm{SO}_{4}{ }^{2-} & .292 \mathrm{E}+01 & .269 \mathrm{E}+01 & .258 \mathrm{E}+01 & .258 \mathrm{E}+01 \\ \mathrm{H}^{+} & .767 \mathrm{E}+01 & .858 \mathrm{E}+01 & .905 \mathrm{E}+01 & .905 \mathrm{E}+01 \\ \mathrm{Tu}^{2+} & .361 \mathrm{E}+01 & .376 \mathrm{E}+01 & .383 \mathrm{E}+01 & .383 \mathrm{E}+01 \\ \mathrm{Fe}^{2+} & .266 \mathrm{E}+00 & .282 \mathrm{E}+00 & .291 \mathrm{E}+00 & .291 \mathrm{E}+00 \\ \mathrm{AgTu}_{2}{ }^{+} & .608 \mathrm{E}-02 & .384 \mathrm{E}-02 & .166 \mathrm{E}-02 & .167 \mathrm{E}-02\end{array}$

$\begin{array}{llcrrrrrr} & & \text { AGt } & \text { AGT+ } & \text { AGT2+ } & \text { AGT3+ } & \text { AGT4+ } & \text { AG2T3+2 } & \text { AG2T6+2 } \\ \text { EN LA SUPER } & .1 \mathrm{E}-11 & .1 \mathrm{E}-05 & .4 \mathrm{E}-04 & .1 \mathrm{E}-03 & .3 \mathrm{E}-05 & .6 \mathrm{E}-02 & .29 \mathrm{E}-05 \\ \text { EN SOLUCION } & .2 \mathrm{E}-12 & .3 \mathrm{E}-06 & .1 \mathrm{E}-04 & .3 \mathrm{E}-04 & .1 \mathrm{E}-05 & .4 \mathrm{E}-03 & .24 \mathrm{E}-06\end{array}$

DIFUSIVIDAD EFECTIVA DE FE+3 $=4.628986969233 \mathrm{E}-10$ CONV $=.66 \mathrm{E}-02 * t+-.23 \mathrm{E}-04 * t * \star 2+.16 \mathrm{E}-07 * t * \star 3$ 
Apéndice D - Experimento 15

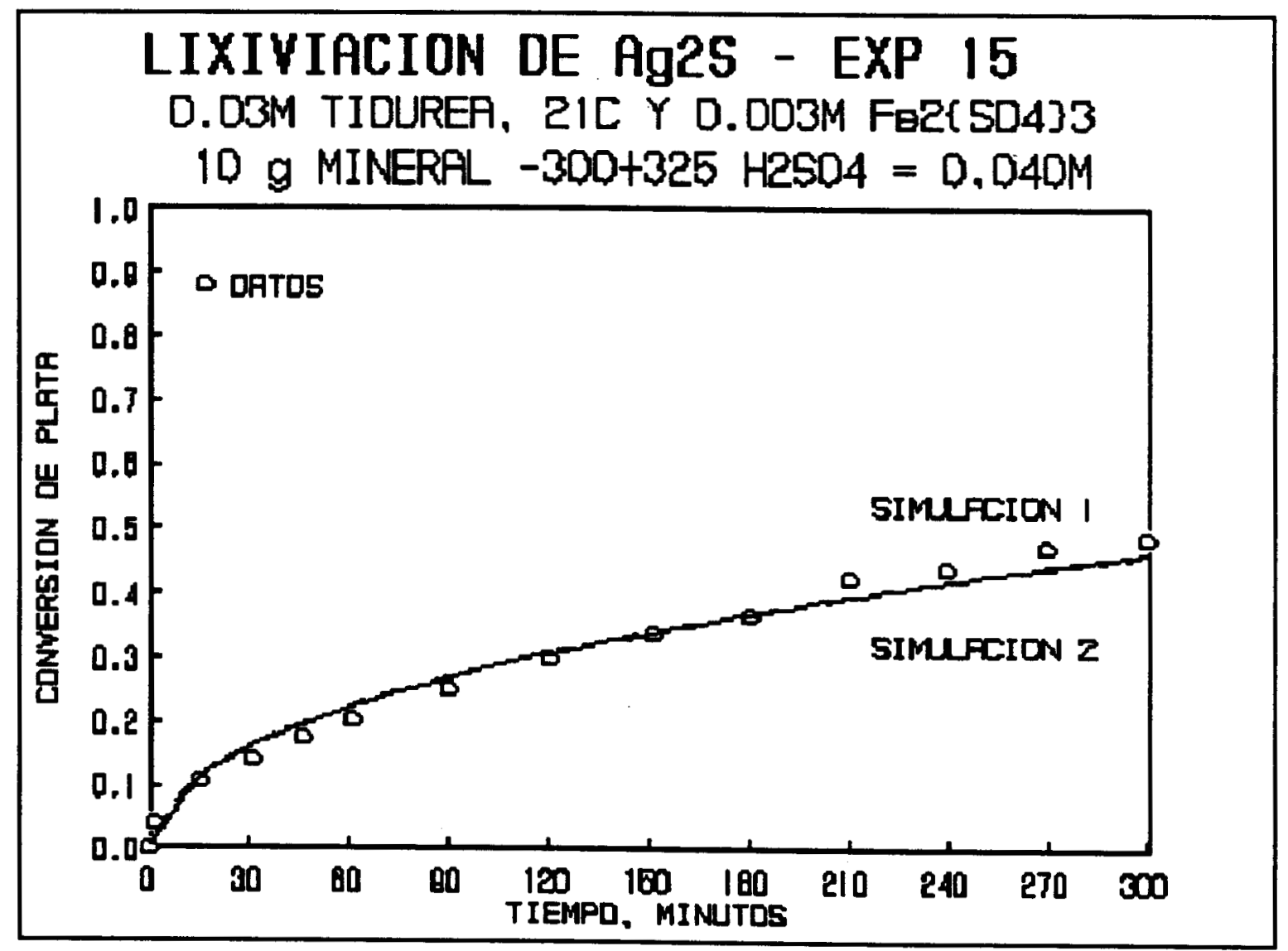




\section{Apéndice D - Experimento 17}

\section{EXPERIMENTO 17}

CONDICIONES :

TEMPERATURA $=35^{\circ} \mathrm{C}$

$$
\begin{aligned}
& \mathrm{Fe}_{2}\left(\mathrm{SO}_{4}\right)_{3}=0.003 \mathrm{M} \\
& \mathrm{CS}^{2}\left(\mathrm{NH}_{2}\right)_{2}=0.10 \mathrm{M} \\
& \mathrm{H}_{2} \mathrm{SO}_{4}=0.040 \mathrm{M}(\mathrm{pH}=1.47) \\
& \text { MINERAL }=10 \mathrm{~g}(-300+325) \\
& \text { SOLUCION }=750 \mathrm{ml}
\end{aligned}
$$

\begin{tabular}{|c|c|c|c|c|c|c|}
\hline $\begin{array}{l}\text { TIEMPO, } \\
\text { minutos }\end{array}$ & $\begin{array}{c}\text { MUESTRA, } \\
\mathrm{ml}\end{array}$ & $\begin{array}{l}\text { LECTURA } \\
\text { Ag }\end{array}$ & $\mathrm{Cu}^{\mathrm{PPM}}$ & $\begin{array}{l}\text { CON } \\
\text { Ag }\end{array}$ & $\begin{array}{l}\text { ION } \\
\mathrm{Cu}\end{array}$ & $\begin{array}{l}E_{h \prime} \\
m V\end{array}$ \\
\hline $\begin{array}{r}1.5 \\
15.0 \\
30.0 \\
45.0 \\
60.0 \\
90.0 \\
120.0 \\
145.0 \\
150.0 \\
180.0 \\
210.0 \\
240.0 \\
270.0 \\
295.0 \\
300.0\end{array}$ & $\begin{array}{l}12 . \\
6 . \\
6 . \\
6 . \\
6 . \\
6 . \\
6 . \\
6 . \\
6 . \\
6 . \\
6 . \\
6 . \\
6 . \\
6 . \\
6 .\end{array}$ & $\begin{array}{l}9.5 \\
32.0 \\
40.0 \\
53.1 \\
61.2 \\
68.2 \\
75.9 \\
80.0 \\
80.3 \\
83.6 \\
85.8 \\
88.1 \\
91.3 \\
91.3 \\
91.3\end{array}$ & $\begin{array}{l}2.4 \\
4.9 \\
5.9 \\
6.3 \\
6.6 \\
6.8 \\
7.1 \\
7.2 \\
7.2 \\
7.3 \\
7.4 \\
7.4 \\
7.5 \\
7.5 \\
7.5\end{array}$ & $\begin{array}{l}.090 \\
.301 \\
.376 \\
.496 \\
.570 \\
.634 \\
.703 \\
.740 \\
.742 \\
.771 \\
.790 \\
.810 \\
.837 \\
.837 \\
.837\end{array}$ & $\begin{array}{l}.057 \\
.116 \\
.139 \\
.148 \\
.155 \\
.159 \\
.166 \\
.168 \\
.168 \\
.171 \\
.173 \\
.173 \\
.175 \\
.175 \\
.175\end{array}$ & $\begin{array}{l}-.190 \\
-.209 \\
-.211 \\
-.214 \\
-.215 \\
-.219 \\
-.220 \\
-.222 \\
-.222 \\
-.224 \\
-.226 \\
-.228 \\
-.229 \\
-.230 \\
-.230\end{array}$ \\
\hline
\end{tabular}

$\alpha=1.0 e^{-4} \mathrm{~min}^{-1}$

$E_{h}$ inicial (ref: sulfato de mercurio) $=-0.134 \mathrm{mV}$

RESIDUO DE Ag $=1.28 \mathrm{~kg} /$ ton

RESIDUO DE Cu $=2.60 \mathrm{~kg} /$ ton

BALANCE METALURGICO DE Ag $=7.88 \mathrm{~kg} / \mathrm{ton}$

BALANCE METALURGICO DE $\mathrm{Cu}=3.15 \mathrm{~kg} / \mathrm{ton}$ 


\title{
Apéndice D - Experimento 17
}

\author{
LIXIVIACION DE ARGENTITA \\ CARACTERISTICAS DEL MINERAL \\ GR POR TON DE AG $=.788 E+04$ \\ DENSIDAD DEL MINERAL $=5.20$ \\ RADIO DE LA PART, $C M=.235 E-02$ \\ POROSIDAD $=0.000 \mathrm{E}+00$ \\ PROPORCION DE SOLIDO A LIQUIDO, KG/IT $=.133 \mathrm{E}-01$
}

CONSTANTES DE EQUILIBRIO A $35.00 \mathrm{C}$

CONCENTRACION INICIAL DE FE2(SO4) $3=.310 E-02$

CONCENTRACION INICIAL DE H2O2 = $0.000 \mathrm{E}+00$

CONCENTRACION INICIAL DE TIOUREA $=.100 \mathrm{E}+00$

$\mathrm{PH}=1.26$

GAMA $=.163 \mathrm{E}-01$

VELOCIDAD DE DESCOMPOSICION DE TIOUREA $=$ TIME* . 100E-03

$\begin{array}{cccrrr}\text { FE+3 } & \text { FESO4+ } & \text { FESO4 }(\mathrm{TH})+ & \text { FEOH+2 } & \text { FE(OH) 2+ } & \text { FE2(OH) } 2+4 \\ .179 E-06 & .885 \mathrm{E}-09 & .620 \mathrm{E}-02 & .206 \mathrm{E}-07 & .336 \mathrm{E}-09 & .602 \mathrm{E}-13 \\ .0000 & .0000 & 1.0000 & .0000 & .0000 & .0000\end{array}$

FUERZA IONICA DE LA SOLUTION $=.074$

CANTIDAD DE ION FERRICO UTILIZABLE $=.620 \mathrm{E}-02$

DIFUSIVIDAD EFECTIVA DE FE+3 = 1.52548840499E-9

ION FERRICO $=-.586 \mathrm{E}-10$

SULFATO LIBRE $=.127 \mathrm{E}-01$

TIOUREA LIBRE $=.938 \mathrm{E}-01$

TOTAL DE ACIDO ANADIDO $=.41 \mathrm{E}-01$

FADS $=.310 E-02$

OXIDANTE CONSUMIDO POR COBRE $=.115 \mathrm{E}-03$

\section{PERFILES ADIMENSIONALES}

TIEMPO DE AJUSTE $=270$.

DIFUSUVIDAD EFECTIVA $=2.53644199083 \mathrm{E}-9$

$\begin{array}{lllll}\mathrm{Fe}^{3+} & .173 \mathrm{E}-09 & .374 \mathrm{E}-08 & .805 \mathrm{E}-08 & .929 \mathrm{E}-08 \\ \mathrm{SO}_{4}{ }^{2-} & .233 \mathrm{E}+01 & .226 \mathrm{E}+01 & .209 \mathrm{E}+01 & .205 \mathrm{E}+01 \\ \mathrm{H}^{+} & .756 \mathrm{E}+01 & .785 \mathrm{E}+01 & .863 \mathrm{E}+01 & .886 \mathrm{E}+01 \\ \mathrm{Tu} & .143 \mathrm{E}+02 & .144 \mathrm{E}+02 & .150 \mathrm{E}+02 & .152 \mathrm{E}+02 \\ \mathrm{Fe}^{2+} & .287 \mathrm{E}+00 & .293 \mathrm{E}+00 & .309 \mathrm{E}+00 & .313 \mathrm{E}+00 \\ \mathrm{AgTu}_{2}{ }^{+} & .393 \mathrm{E}-01 & .334 \mathrm{E}-01 & .126 \mathrm{E}-01 & .346 \mathrm{E}-04\end{array}$


Apéndice D - Experimento 17

$\begin{array}{llcrrrrrr} & & \text { AGt } & \text { AGT } & \text { AGT2+ } & \text { AGT3+ } & \text { AGT4+ } & \text { AG2T3+2 } & \text { AG2T6+2 } \\ \text { EN IA SUPER } & .3 E-11 & .7 \mathrm{E}-06 & .2 \mathrm{E}-03 & .3 \mathrm{E}-02 & .2 \mathrm{E}-03 & .2 \mathrm{E}-02 & .19 \mathrm{E}-02 \\ \text { EN SOLUCION } & .2 \mathrm{E}-14 & .6 \mathrm{E}-09 & .2 \mathrm{E}-06 & .3 \mathrm{E}-05 & .2 \mathrm{E}-06 & .1 \mathrm{E}-08 & .16 \mathrm{E}-08\end{array}$

$\begin{array}{cc}\text { ADIM. TIEMPO } & \text { RC/RP } \\ .003 & .991 \mathrm{E}+00 \\ .053 & .965 \mathrm{E}+00 \\ .083 & .953 \mathrm{E}+00 \\ .113 & .947 \mathrm{E}+00 \\ .143 & .941 \mathrm{E}+00 \\ .173 & .936 \mathrm{E}+00 \\ .203 & .931 \mathrm{E}+00 \\ .303 & .909 \mathrm{E}+00 \\ .403 & .898 \mathrm{E}+00 \\ .503 & .887 \mathrm{E}+00 \\ .603 & .878 \mathrm{E}+00\end{array}$

TIEMPO, MIN

$.116 \mathrm{E}+00$

$.193 \mathrm{E}+01$

$.302 \mathrm{E}+01$

$.411 \mathrm{E}+01$

$.520 E+01$

$.629 \mathrm{E}+01$

$.737 E+01$

$.110 \mathrm{E}+02$

$.146 \mathrm{E}+02$

$.183 \mathrm{E}+02$

$.219 \mathrm{E}+02$
$\mathrm{Fe}^{3+}$

$\mathrm{SO}^{+2-}$

Tu

$\mathrm{Fe}^{2+}$

$\mathrm{AgTu}_{2}^{+}$
$.174 \mathrm{E}-09$

.38

\begin{abstract}
$.387 \mathrm{E}-08$
\end{abstract}
$.797 \mathrm{E}-08$

$.210 E+01$

$.861 \mathrm{E}+01$

$.149 \mathrm{E}+02$

$.309 \mathrm{E}+00$

$.131 \mathrm{E}-01$
CONVERSION

$.225 \mathrm{E}-01$

$.937 \mathrm{E}-01$

$.117 \mathrm{E}+00$

$.136 \mathrm{E}+00$

$.153 E+00$

$.169 \mathrm{E}+00$

$.183 \mathrm{E}+00$

$.220 \mathrm{E}+00$

$.252 \mathrm{E}+00$

$.280 \mathrm{E}+00$

$.304 \mathrm{E}+00$

$.906 \mathrm{E}-08$

$.206 E+01$

$.882 \mathrm{E}+01$

$.151 \mathrm{E}+02$

$.313 \mathrm{E}+00$

.308E-02
PH NUEVO

1.47

1.47

1.47

1.47

1.47

1.47

1.47

1.47

1.47

1.47

1.47

EN IA SUPER .3E-11 .7E-06

AG+ AGT+ AGT2+

AGT3+

AGT4+ AG2T3+2 AG2T6+2

EN SOLUCION .2E-12 .6E-07

. $2 \mathrm{E}-03$

$.3 \mathrm{E}-02$

$.2 \mathrm{E}-03$

$.2 \mathrm{E}-02 \quad .18 \mathrm{E}-02$

FesO4Tu = $.38 \mathrm{E}-07$

$\begin{array}{cc}\text { ADIM. TIEMPO } & \text { RC/RP } \\ .903 & .846 \mathrm{E}+00 \\ 1.103 & .831 \mathrm{E}+00 \\ 1.303 & .818 \mathrm{E}+00 \\ 1.503 & .805 \mathrm{E}+00 \\ 1.703 & .792 \mathrm{E}+00 \\ 2.003 & .770 \mathrm{E}+00 \\ 2.303 & .754 \mathrm{E}+00 \\ 2.603 & .739 \mathrm{E}+00 \\ 2.903 & .724 \mathrm{E}+00 \\ 3.203 & .710 \mathrm{E}+00 \\ 3.803 & .672 \mathrm{E}+00 \\ 4.403 & .646 \mathrm{E}+00 \\ 5.003 & .622 \mathrm{E}+00 \\ 5.603 & .598 \mathrm{E}+00 \\ 6.203 & .574 \mathrm{E}+00 \\ 7.203 & .524 \mathrm{E}+00 \\ 8.203 & .486 \mathrm{E}+00\end{array}$

TIEMPO, MIN

$.328 E+02$

$.400 \mathrm{E}+02$

$.473 \mathrm{E}+02$

$.545 \mathrm{E}+02$

$.618 \mathrm{E}+02$

$.727 \mathrm{E}+02$

$.836 \mathrm{E}+02$

$.945 \mathrm{E}+02$

$.105 \mathrm{E}+03$

$.116 \mathrm{E}+03$

$.138 \mathrm{E}+03$

$.160 E+03$

$.182 E+03$

$.203 E+03$

$.225 \mathrm{E}+03$

$.261 E+03$

$.298 E+03$
CONVERSION

$.366 \mathrm{E}+00$

$.400 E+00$

$.430 \mathrm{E}+00$

$.457 \mathrm{E}+00$

$.483 \mathrm{E}+00$

$.517 \mathrm{E}+00$

$.547 \mathrm{E}+00$

$.575 \mathrm{E}+00$

$.601 E+00$

$.624 \mathrm{E}+00$

$.666 \mathrm{E}+00$

$.703 E+00$

$.735 \mathrm{E}+00$

$.765 \mathrm{E}+00$

$.791 \mathrm{E}+00$

$.829 E+00$

$.862 \mathrm{E}+00$
PH NUEVO

1.47

1.47

1.47

1.47

1.47

1.47

1.47

1.47

1.47

1.47

1.47

1.47

1.47

1.47

1.47

1.47

1.47

177 
Apéndice D - Experimento 17

\begin{tabular}{lccccc}
\multicolumn{2}{c}{9.203} & $.449 \mathrm{E}+00$ & $.334 \mathrm{E}+03$ & $.890 \mathrm{E}+00$ & 1.47 \\
$\mathrm{Fe}^{3+}{ }^{10.203}$ & $.410 \mathrm{E}+00$ & $.370 \mathrm{E}+03$ & $.914 \mathrm{E}+00$ & 1.47 \\
$\mathrm{SO}_{4}{ }^{2-}$ & $.191 \mathrm{E}-09$ & $.537 \mathrm{E}-08$ & $.848 \mathrm{E}-08$ & $.892 \mathrm{E}-08$ & \\
$\mathrm{H}^{+}$ & $.232 \mathrm{E}+01$ & $.220 \mathrm{E}+01$ & $.209 \mathrm{E}+01$ & $.207 \mathrm{E}+01$ & \\
$\mathrm{Tu}^{2+}$ & $.759 \mathrm{E}+01$ & $.810 \mathrm{E}+01$ & $.866 \mathrm{E}+01$ & $.874 \mathrm{E}+01$ \\
$\mathrm{Fe}^{2+}$ & $.136 \mathrm{E}+02$ & $.139 \mathrm{E}+02$ & $.142 \mathrm{E}+02$ & $.143 \mathrm{E}+02$ \\
$\mathrm{AgTu}_{2}{ }^{+}$ & $.288 \mathrm{E}+00$ & $.299 \mathrm{E}+00$ & $.310 \mathrm{E}+00$ & $.311 \mathrm{E}+00$ & \\
& $.390 \mathrm{E}-01$ & $.282 \mathrm{E}-01$ & $.113 \mathrm{E}-01$ & $.752 \mathrm{E}-02$ &
\end{tabular}

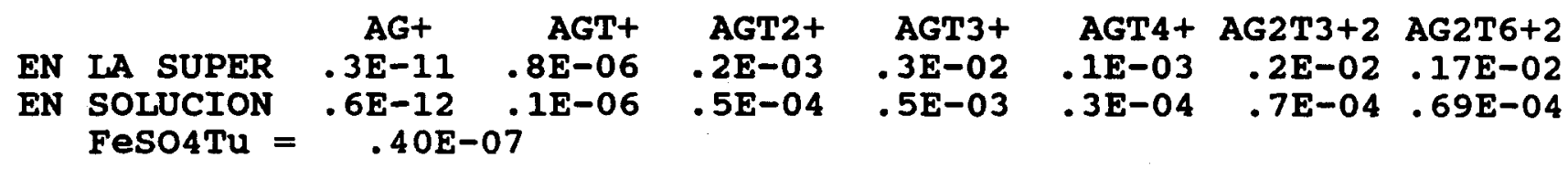

ADIM. TIEMPO

14.203

17.203

$\mathrm{Fe}^{3+}$

$\mathrm{SO}_{4}^{2-}$

$\mathrm{H}^{+}$

$\mathrm{Tu}$

$\mathrm{Fe}^{2+}$

$\mathrm{AgTu}_{2}^{+}$
$\mathrm{RC} / \mathrm{RP}$

$.138 \mathrm{E}+00$

$.619 \mathrm{E}-01$
TIEMPO, MIN

$.515 E+03$

$.624 \mathrm{E}+03$
CONVERSION

$.983 \mathrm{E}+00$

$.997 \mathrm{E}+00$
PH NUEVO

1.47

1.47

$\begin{array}{llcrrrrrr} & & \text { AGt } & \text { AGT+ } & \text { AGT2+ } & \text { AGT3+ } & \text { AGT4+ } & \text { AG2T3+2 } & \text { AG2T6+2 } \\ \text { EN LA SUPER } & .3 \mathrm{E}-11 & .7 \mathrm{E}-06 & .2 \mathrm{E}-03 & .2 \mathrm{E}-02 & .1 \mathrm{E}-03 & .2 \mathrm{E}-02 & .12 \mathrm{E}-02 \\ \text { EN SOLUCION } & .6 \mathrm{E}-12 & .2 \mathrm{E}-06 & .5 \mathrm{E}-04 & .6 \mathrm{E}-03 & .3 \mathrm{E}-04 & .8 \mathrm{E}-04 & .74 \mathrm{E}-04\end{array}$

DIFUSIVIDAD EFECTIVA DE FE+3 $=2.53644199083 \mathrm{E}-9$

CONV $=.36 \mathrm{E}-01 * t+-.68 \mathrm{E}-03 * t * * 2+.24 \mathrm{E}-05 * t * * 3$ 


\section{Apéndice D - Experimento 17}

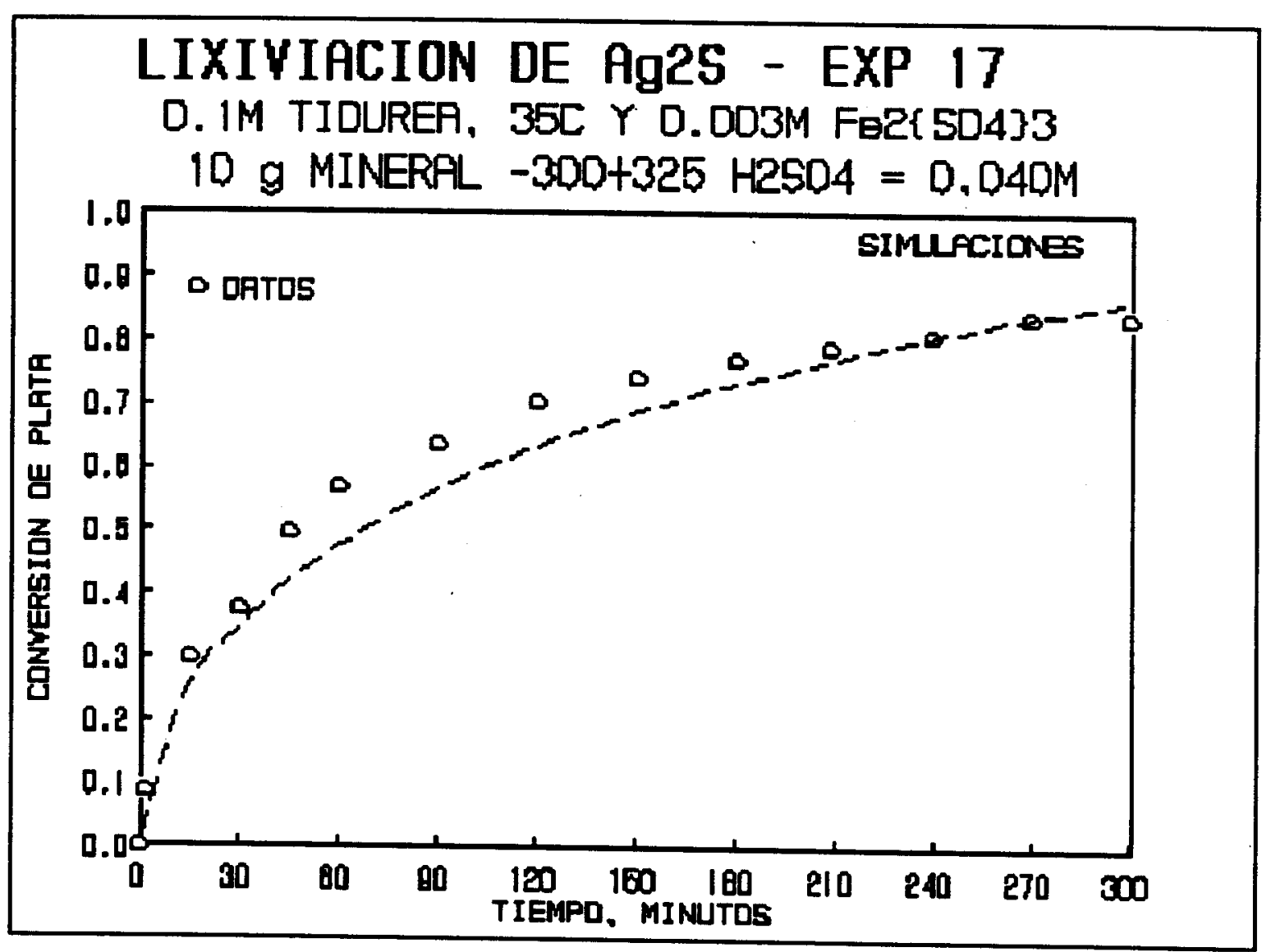


Apéndice D - Experimento 18

\section{EXPERIMENTO 18}

\section{CONDICIONES :}

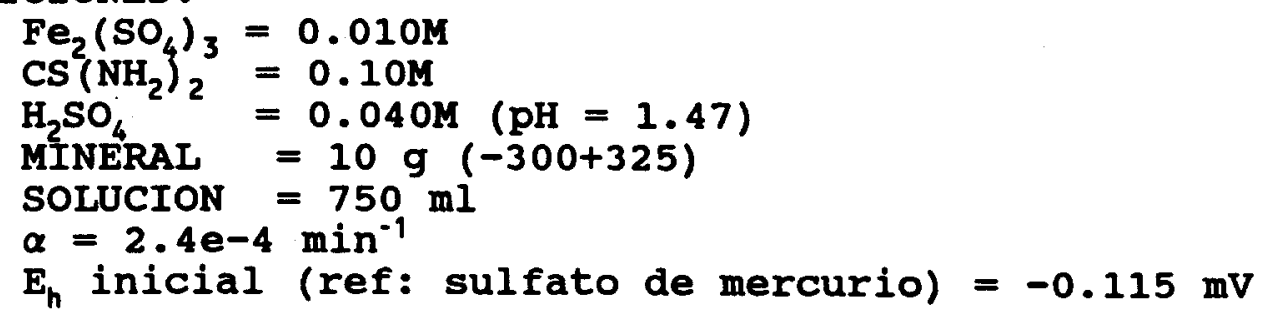

\begin{tabular}{rcccccc} 
TIEMPO, & MUESTRA, & \multicolumn{2}{c}{ LECTURA, PPM } & \multicolumn{2}{c}{ CONVERSION } & E $_{\text {h' }}$ \\
minutos & ml & Ag & Cu & Ag & Cu & mV \\
1.0 & 12. & 9.0 & 2.6 & .092 & .063 & -.143 \\
15.0 & 6. & 26.4 & 5.3 & .267 & .128 & -.199 \\
31.0 & 6. & 37.2 & 6.2 & .375 & .149 & -.200 \\
45.0 & 6. & 44.4 & 6.7 & .446 & .161 & -.201 \\
60.0 & 6. & 49.5 & 7.1 & .496 & .170 & -.201 \\
90.0 & 6. & 55.0 & 7.4 & .550 & .177 & -.201 \\
120.0 & 6. & 62.7 & 7.6 & .624 & .182 & -.202 \\
145.0 & 6. & 66.5 & 7.7 & .661 & .184 & -.203 \\
150.0 & 6. & 67.1 & 7.7 & .666 & .184 & -.203 \\
181.0 & 6. & 69.3 & 7.8 & .687 & .186 & -.204 \\
211.0 & 6. & 70.4 & 7.8 & .697 & .186 & -.205 \\
240.0 & 6. & 72.6 & 7.9 & .718 & .188 & -.206 \\
271.0 & 6. & 74.8 & 7.9 & .738 & .188 & -.206 \\
295.0 & 6. & 75.7 & 8.0 & .746 & .190 & -.207 \\
305.0 & 6. & 75.9 & 8.0 & .748 & .190 & -.207
\end{tabular}

RESIDUO DE Ag $=1.85 \mathrm{~kg} /$ ton

RESIDUO DE $\mathrm{Cu}=2.50 \mathrm{~kg} /$ ton

BALANCE METALURGICO DE Ag $=7.33 \mathrm{~kg} /$ ton

BALANCE METALURGICO DE $\mathrm{Cu}=3.09 \mathrm{~kg} /$ ton 
Apéndice D - Experimento 18

LIXIVIACION DE ARGENTITA

CARACTERISTICAS DEL MINERAL

GR POR TON DE AG $=.733 E+04$

DENSIDAD DEL MINERAL $=5.20$

RADIO DE LA PART, CM $=.235 \mathrm{E}-02$

POROSIDAD $=0.000 \mathrm{E}+00$

PROPORCION DE SOLIDO A LIQUIDO, KG/LT $=.133 E-01$

CONSTANTES DE EQUILIBRIO A $21.00 \mathrm{C}$

CONCENTRACION INICIAL DE FE2(SO4) $3=.103 E-01$

CONCENTRACION INICIAL DE H2O2 $=0.000 \mathrm{E}+00$

CONCENTRACION INICIAL DE TIOUREA $=.100 \mathrm{E}+00$

GAMA $=.584 \mathrm{E}-01$

VELOCIDAD DE DESCOMPOSICION DE TIOUREA = TIME* .240E-03

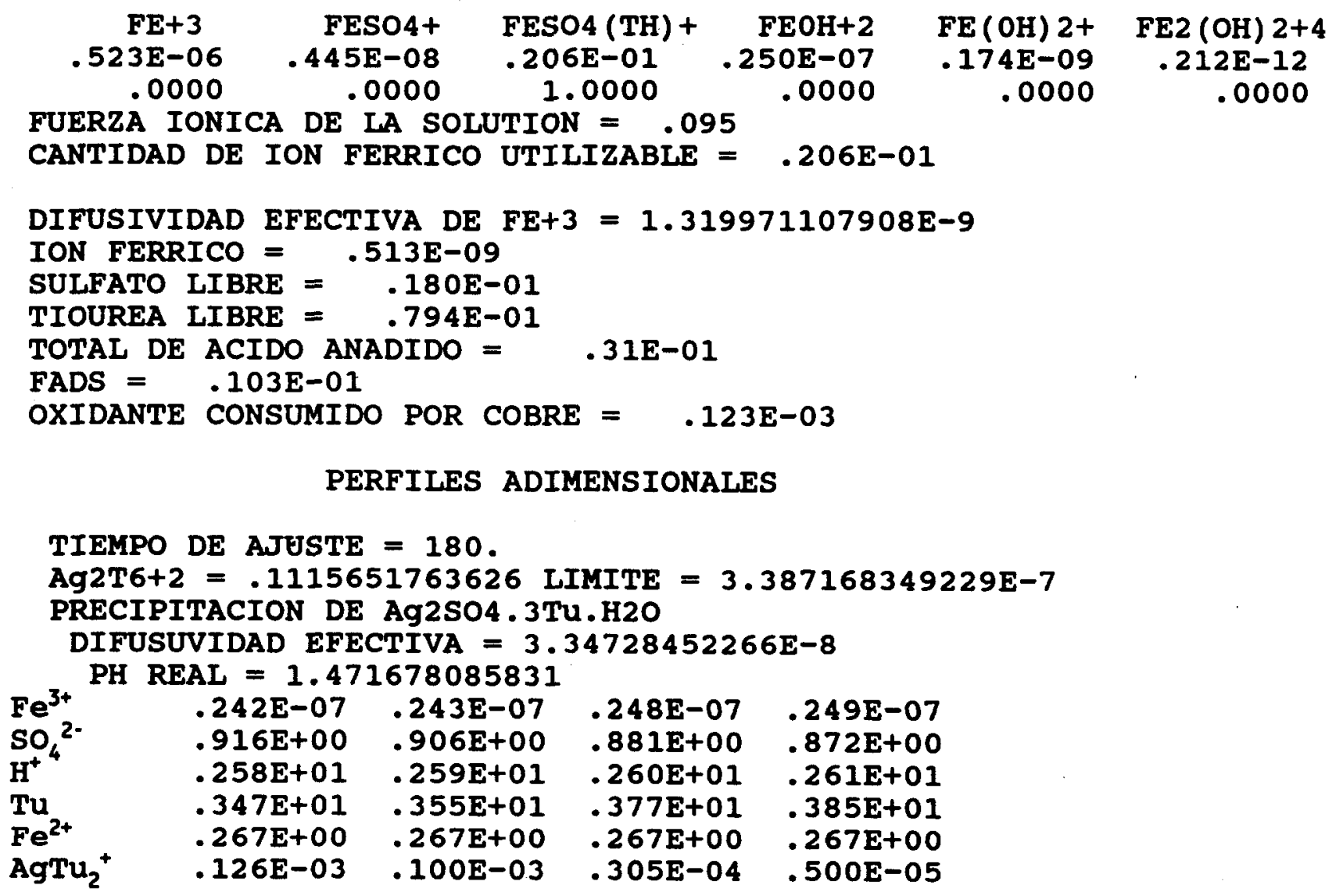


Apéndice D - Experimento 18

$\begin{array}{llcrrrrrr} & & \text { AGt } & \text { AGT+ } & \text { AGT2+ } & \text { AGT3+ } & \text { AGT4+ } & \text { AG2T3+2 } & \text { AG2T6+2 } \\ \text { EN IA SUPER } & .7 \mathrm{E}-14 & .2 \mathrm{E}-07 & .3 \mathrm{E}-05 & .3 \mathrm{E}-04 & .2 \mathrm{E}-05 & .9 \mathrm{E}-05 & .14 \mathrm{E}-06 \\ \text { EN SOLUCION } & .2 \mathrm{E}-15 & .9 \mathrm{E}-09 & .1 \mathrm{E}-06 & .1 \mathrm{E}-05 & .1 \mathrm{E}-06 & .1 \mathrm{E}-07 & .27 \mathrm{E}-09\end{array}$

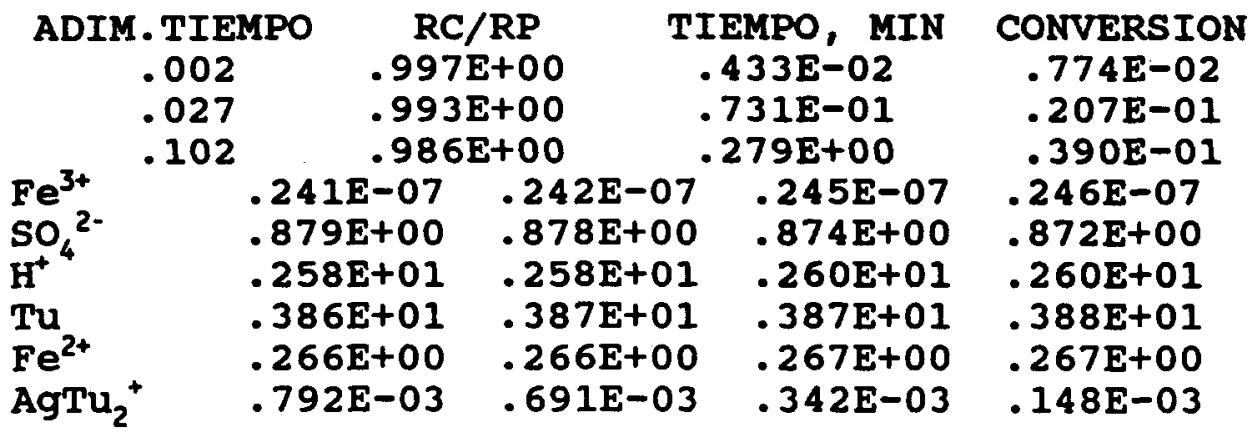

PH NUEVO

1.47

1.47

1.47

$\begin{array}{llcrrrrrr} & & \text { AGt } & \text { AGTt } & \text { AGT2+ } & \text { AGT3+ } & \text { AGT4+ } & \text { AG2T3+2 } & \text { AG2T6+2 } \\ \text { EN IA SUPER } & .3 \mathrm{E}-13 & .1 \mathrm{E}-06 & .2 \mathrm{E}-04 & .2 \mathrm{E}-03 & .2 \mathrm{E}-04 & .3 \mathrm{E}-03 & .67 \mathrm{E}-05 \\ \text { EN SOLUCION } & .6 \mathrm{E}-14 & .3 \mathrm{E}-07 & .3 \mathrm{E}-05 & .3 \mathrm{E}-04 & .3 \mathrm{E}-05 & .1 \mathrm{E}-04 & .24 \mathrm{E}-06 \\ \text { FeSO4Tu }= & .17 \mathrm{E}-04 & & & & & & \end{array}$

ADIM. TIEMPO

.752

.852

1.002

1.152

1.302

1.452

1.602

1.902

2.202

2.502

2.802

3.102

3.602

4.102

4.602

5.102
$\mathrm{Fe}^{3+}$

Tu

$\mathrm{Fe}^{2+}$

$\mathrm{AgTu}_{2}^{+}$
RC/RP

$.963 E+00$

$.957 \mathrm{E}+00$

$.954 \mathrm{E}+00$

$.952 \mathrm{E}+00$

$.950 \mathrm{E}+00$

$.947 \mathrm{E}+00$

$.941 \mathrm{E}+00$

$.938 \mathrm{E}+00$

$.934 \mathrm{E}+00$

$.931 E+00$

$.928 \mathrm{E}+00$

$.921 \mathrm{E}+00$

$.917 \mathrm{E}+00$

$.912 \mathrm{E}+00$

$.908 \mathrm{E}+00$
$.961 \mathrm{E}+00$

TIEMPO, MIN

$.207 \mathrm{E}+01$

$.234 \mathrm{E}+01$

$.275 \mathrm{E}+01$

$.317 \mathrm{E}+01$

$.358 \mathrm{E}+01$

$.399 \mathrm{E}+01$

$.440 \mathrm{E}+01$

$.523 E+01$

$.605 \mathrm{E}+01$

$.688 \mathrm{E}+01$

$.770 \mathrm{E}+01$

$.853 \mathrm{E}+01$

$.990 \mathrm{E}+01$

. 113E+02

.127E+02

. $140 \mathrm{E}+02$

$.241 \mathrm{E}-07 \quad .242 \mathrm{E}-07$

$.879 \mathrm{E}+00 \quad .878 \mathrm{E}+00$

$.258 \mathrm{E}+01 \quad .258 \mathrm{E}+01$

$.384 \mathrm{E}+01$. $384 \mathrm{E}+01$

$.266 \mathrm{E}+00$

$.797 \mathrm{E}-03$
$.266 \mathrm{E}+00$

$.714 \mathrm{E}-03$
$.245 \mathrm{E}-07$

$.875 \mathrm{E}+00$

$.259 \mathrm{E}+01$

$.385 \mathrm{E}+01$

$.267 E+00$

$.460 \mathrm{E}-03$
CONVERSION

.102E+00

$.108 \mathrm{E}+00$

$.116 \mathrm{E}+00$

$.124 \mathrm{E}+00$

$.131 E+00$

$.138 \mathrm{E}+00$

$.145 \mathrm{E}+00$

$.156 \mathrm{E}+00$

$.167 \mathrm{E}+00$

$.177 \mathrm{E}+00$

$.186 \mathrm{E}+00$

$.195 \mathrm{E}+00$

$.208 \mathrm{E}+00$

$.220 \mathrm{E}+00$

$.232 \mathrm{E}+00$

$.242 E+00$

$.246 \mathrm{E}-07$

$.874 \mathrm{E}+00$

$.260 \mathrm{E}+01$

$.385 \mathrm{E}+01$

$.267 \mathrm{E}+00$

$.349 \mathrm{E}-03$
PH NUEVO

1.47

1.47

1.47

1.47

1.47

1.47

1.47

1.47

1.47

1.47

1.47

1.47

1.47

1.47

1.47

1.47 
Apéndice D - Experimento 18

$\begin{array}{llcrrrrrr} & & \text { AGt } & \text { AGT+ } & \text { AGT2+ } & \text { AGT3+ } & \text { AGT4+ } & \text { AG2T3+2 } & \text { AG2T6+2 } \\ \text { EN IA SUPER } & .4 \mathrm{E}-13 & .1 \mathrm{E}-06 & .2 \mathrm{E}-04 & .2 \mathrm{E}-03 & .2 \mathrm{E}-04 & .3 \mathrm{E}-03 & .68 \mathrm{E}-05 \\ \text { EN SOLUCION } & .2 \mathrm{E}-13 & .6 \mathrm{E}-07 & .7 \mathrm{E}-05 & .05 \mathrm{E}-04 & .7 \mathrm{~F}-05 & .7 \mathrm{E}-04 & .13 \mathrm{E}-05\end{array}$

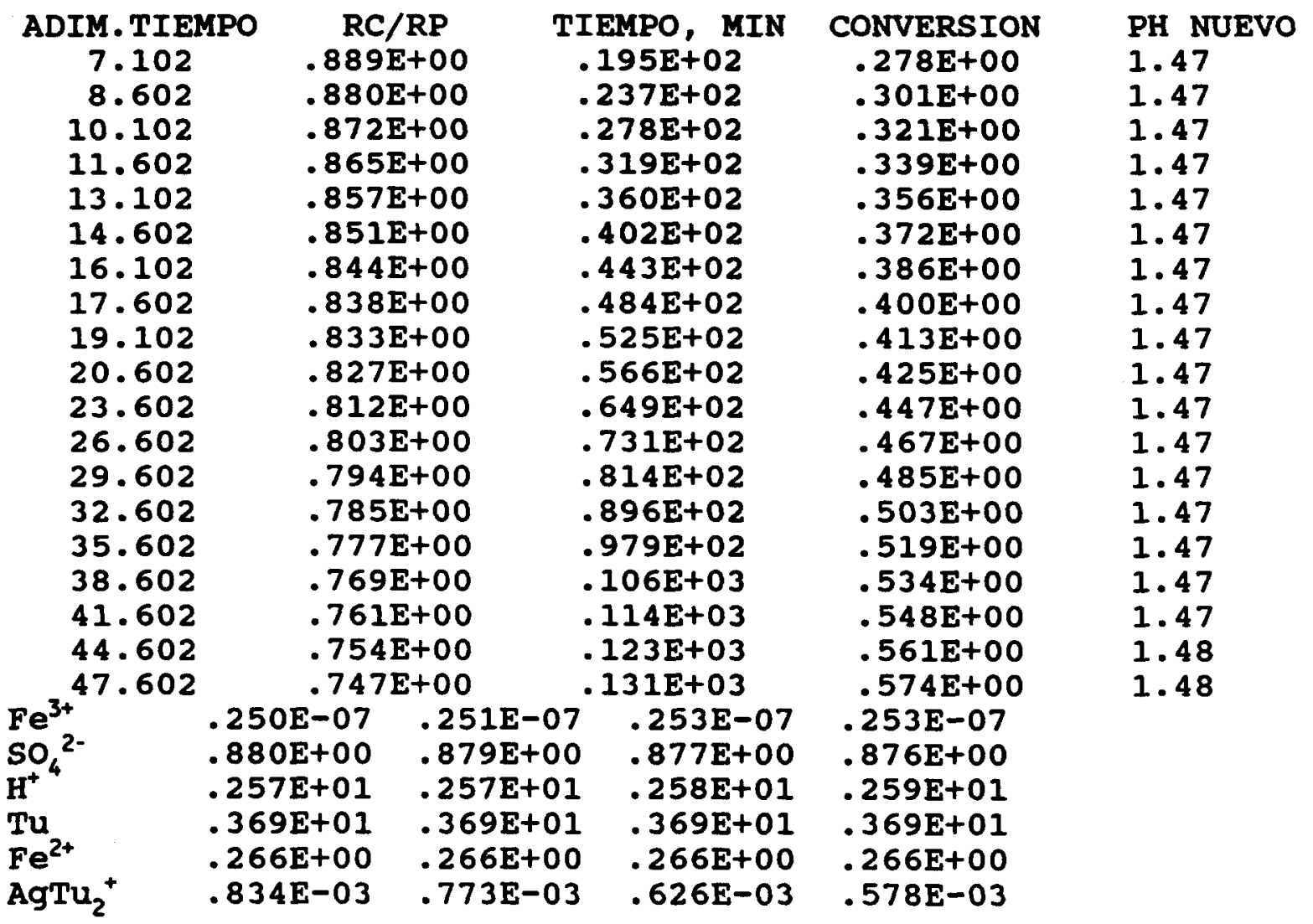

$\begin{array}{llcrrrrrr} & \text { AGt } & \text { AGT+ } & \text { AGT2+ } & \text { AGT3+ } & \text { AGT4+ } & \text { AG2T3+2 } & \text { AG2T6+2 } \\ \text { EN IA SUPER } & .4 \mathrm{E}-13 & .2 \mathrm{E}-06 & .2 \mathrm{E}-04 & .2 \mathrm{E}-03 & .2 \mathrm{E}-04 & .4 \mathrm{E}-03 & .68 \mathrm{E}-05 \\ \text { EN SOLUCION } & .3 \mathrm{E}-13 & .1 \mathrm{E}-06 & .1 \mathrm{E}-04 & .1 \mathrm{E}-03 & .1 \mathrm{E}-04 & .2 \mathrm{E}-03 & .33 \mathrm{E}-05 \\ \text { FeSO4TU }= & .17 \mathrm{E}-04 & & & & & \end{array}$

$\begin{array}{cc}\text { ADIM.TIEMPO } & \text { RC/RP } \\ 55.602 & .725 \mathrm{E}+00 \\ 60.602 & .714 \mathrm{E}+00 \\ 65.602 & .704 \mathrm{E}+00 \\ 70.602 & .693 \mathrm{E}+00 \\ 75.602 & .683 \mathrm{E}+00 \\ 80.602 & .674 \mathrm{E}+00 \\ 85.602 & .664 \mathrm{E}+00 \\ 90.602 & .655 \mathrm{E}+00 \\ 95.602 & .645 \mathrm{E}+00\end{array}$

TIEMPO, MIN
$.153 E+03$
$.167 E+03$
$.180 E+03$
$.194 E+03$
$.208 E+03$
$.222 E+03$
$.235 E+03$
$.249 E+03$
$.263 E+03$

PH NUEVO

1.48

1.48

1.48

1.48

1.48

1.48

1.48

1.48

1.48

183 


\section{Apéndice D - Experimento 18}

$$
\begin{aligned}
& 100.602 \\
& 110.602 \\
& 120.602 \\
& 130.602 \\
& 140.602 \\
& 150.602 \\
& 160.602 \\
& 170.602 \\
& 180.602 \\
& 190.602 \\
& 200.602 \\
& 215.602 \\
& 230.602 \\
& 245.602 \\
& 260.602 \\
& 275.602
\end{aligned}
$$

$$
\begin{aligned}
& .636 \mathrm{E}+00 \\
& .609 \mathrm{E}+00 \\
& .591 \mathrm{E}+00 \\
& .572 \mathrm{E}+00 \\
& .553 \mathrm{E}+00 \\
& .534 \mathrm{E}+00 \\
& .514 \mathrm{E}+00 \\
& .494 \mathrm{E}+00 \\
& .473 \mathrm{E}+00 \\
& .452 \mathrm{E}+00 \\
& .429 \mathrm{E}+00 \\
& .381 \mathrm{E}+00 \\
& .339 \mathrm{E}+00 \\
& .291 \mathrm{E}+00 \\
& .233 \mathrm{E}+00 \\
& .154 \mathrm{E}+00
\end{aligned}
$$

$\begin{array}{lll}.277 \mathrm{E}+03 & .734 \mathrm{E}+00 & 1.48 \\ .304 \mathrm{E}+03 & .756 \mathrm{E}+00 & 1.48 \\ .332 \mathrm{E}+03 & .778 \mathrm{E}+00 & 1.48 \\ .359 \mathrm{E}+03 & .798 \mathrm{E}+00 & 1.48 \\ .387 \mathrm{E}+03 & .817 \mathrm{E}+00 & 1.48 \\ .414 \mathrm{E}+03 & .834 \mathrm{E}+00 & 1.48 \\ .442 \mathrm{E}+03 & .851 \mathrm{E}+00 & 1.48 \\ .469 \mathrm{E}+03 & .867 \mathrm{E}+00 & 1.48 \\ .497 \mathrm{E}+03 & .883 \mathrm{E}+00 & 1.48 \\ .524 \mathrm{E}+03 & .897 \mathrm{E}+00 & 1.48 \\ .552 \mathrm{E}+03 & .911 \mathrm{E}+00 & 1.48 \\ .593 \mathrm{E}+03 & .931 \mathrm{E}+00 & 1.48 \\ .634 \mathrm{E}+03 & .949 \mathrm{E}+00 & 1.48 \\ .675 \mathrm{E}+03 & .965 \mathrm{E}+00 & 1.48 \\ .717 \mathrm{E}+03 & .979 \mathrm{E}+00 & 1.48 \\ .758 \mathrm{E}+03 & .991 \mathrm{E}+00 & 1.48\end{array}$

$\begin{array}{lllll}\mathrm{Fe}^{3+} & .302 \mathrm{E}-07 & .312 \mathrm{E}-07 & .311 \mathrm{E}-07 & .310 \mathrm{E}-07 \\ \mathrm{SO}_{4}{ }^{2-} & .886 \mathrm{E}+00 & .877 \mathrm{E}+00 & .878 \mathrm{E}+00 & .879 \mathrm{E}+00 \\ \mathrm{H}^{+} & .254 \mathrm{E}+01 & .258 \mathrm{E}+01 & .258 \mathrm{E}+01 & .257 \mathrm{E}+01 \\ \mathrm{Tu}^{2+} & .296 \mathrm{E}+01 & .297 \mathrm{E}+01 & .297 \mathrm{E}+01 & .296 \mathrm{E}+01 \\ \mathrm{Fe}^{2+} & .264 \mathrm{E}+00 & .266 \mathrm{E}+00 & .266 \mathrm{E}+00 & .266 \mathrm{E}+00 \\ \mathrm{AgTu}_{2}{ }^{+} & .105 \mathrm{E}-02 & .659 \mathrm{E}-03 & .667 \mathrm{E}-03 & .732 \mathrm{E}-03\end{array}$

$\begin{array}{llcrrrrrr} & & \text { AG+ } & \text { AGT+ } & \text { AGT2+ } & \text { AGT3+ } & \text { AGT4+ } & \text { AG2T3+2 } & \text { AG2T6+2 } \\ \text { EN LA SUPER } & .8 \mathrm{E}-13 & .2 \mathrm{E}-06 & .2 \mathrm{E}-04 & .2 \mathrm{E}-03 & .1 \mathrm{E}-04 & .7 \mathrm{E}-03 & .70 \mathrm{E}-05 \\ \text { EN SOLUCION } & .5 \mathrm{E}-13 & .2 \mathrm{E}-06 & .02 \mathrm{E}-04 & .1 \mathrm{E}-03 & .9 \mathrm{E}-05 & .4 \mathrm{E}-03 & .35 \mathrm{E}-05\end{array}$

DIFUSIVIDAD EFECTIVA DE FE+3 $=3.34728452266 E-8$ CONV $=.35 \mathrm{E}-01 * t+-.51 \mathrm{E}-03 * t * * 2+.12 \mathrm{E}-05 * t * * 3$ 


\section{Apéndice D - Experimento 18}

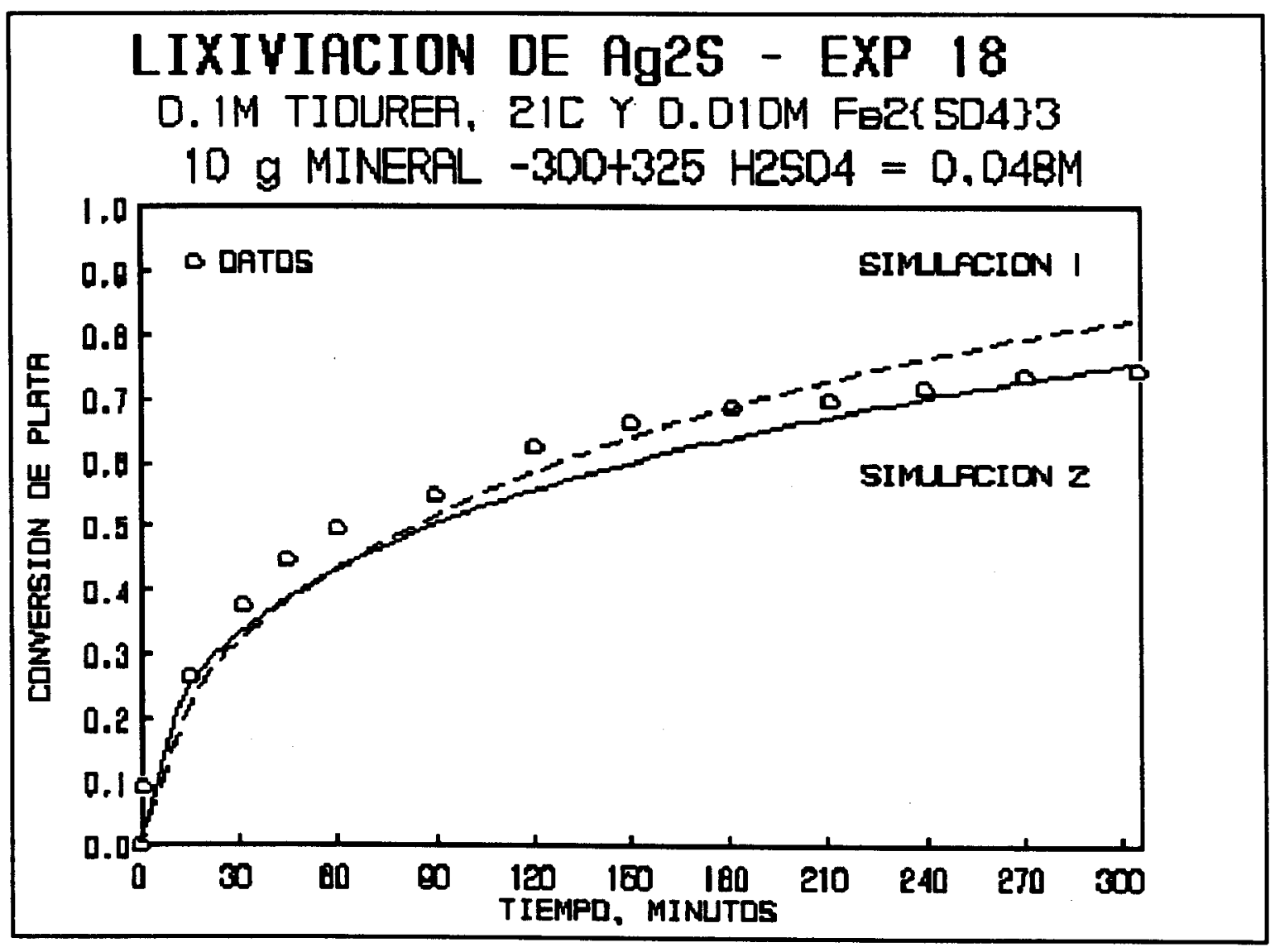




\section{Apéndice D - Experimento 19}

\section{EXPERIMENTO 19}

CONDICIONES :

TEMPERATURA $=3^{\circ} \mathrm{C}$

$$
\begin{aligned}
& \mathrm{Fe}_{2}\left(\mathrm{SO}_{4}\right)_{3}=0.003 \mathrm{M} \\
& \mathrm{CS}\left(\mathrm{NH}_{2}\right)_{2}=0.10 \mathrm{M} \\
& \mathrm{H}_{2} \mathrm{SO}_{4}=0.048 \mathrm{M}(\mathrm{pH}=1.48) \\
& \mathrm{MINERAL}=10 \mathrm{~g}(-300+325)
\end{aligned}
$$$$
\text { SOLUCION }=750 \mathrm{ml}
$$

$\alpha=1.3 \mathrm{e}^{-4} \mathrm{~min}^{-1}$

$E_{h}$ inicial (ref: sulfato de mercurio) $=-0.120 \mathrm{mV} i$ ?

\begin{tabular}{rcccccc} 
TIEMPO, & MUESTRA, & \multicolumn{2}{c}{ LECTURA, PPM } & \multicolumn{2}{c}{ CONVERSION } & E $_{h \prime}$ \\
minutos & $\mathrm{ml}$ & $\mathrm{Ag}$ & $\mathrm{Cu}$ & $\mathrm{Ag}$ & $\mathrm{Cu}$ & $\mathrm{mV}$ \\
1.0 & 12. & 3.3 & 1.2 & .035 & .030 & -.179 \\
15.0 & 6. & 12.5 & 3.0 & .132 & .074 & -.232 \\
30.0 & 6. & 16.0 & 3.8 & .168 & .093 & -.232 \\
45.0 & 6. & 17.5 & 4.3 & .184 & .105 & -.232 \\
60.0 & 6. & 20.5 & 4.7 & .215 & .115 & -.232 \\
90.0 & 6. & 24.5 & 5.4 & .255 & .131 & -.232 \\
120.0 & 6. & 27.5 & 5.8 & .286 & .141 & -.232 \\
145.0 & 6. & 28.5 & 6.2 & .296 & .150 & -.233 \\
150.0 & 6. & 28.5 & 6.2 & .296 & .150 & -.233 \\
180.0 & 6. & 31.2 & 6.5 & .322 & .157 & -.233 \\
210.0 & 6. & 31.8 & 6.7 & .328 & .161 & -.233 \\
244.0 & 6. & 32.0 & 6.9 & .330 & .166 & -.233 \\
274.0 & 6. & 32.0 & 7.0 & .330 & .168 & -.233 \\
304.0 & 6. & 32.6 & 7.2 & .336 & .172 & -.234 \\
310.0 & 6. & 32.8 & 7.2 & .338 & .172 & -.234
\end{tabular}

RESIDUO DE Ag $=4.65 \mathrm{~kg} /$ ton

RESIDUO DE $\mathrm{Cu}=2.50 \mathrm{~kg} /$ ton

BALANCE METALURGICO DE Ag $=7.02 \mathrm{~kg} / \mathrm{ton}$

BALANCE METALURGICO DE $\mathrm{Cu}=3.02 \mathrm{~kg} /$ ton 


\section{Apéndice D - Experimento 19}

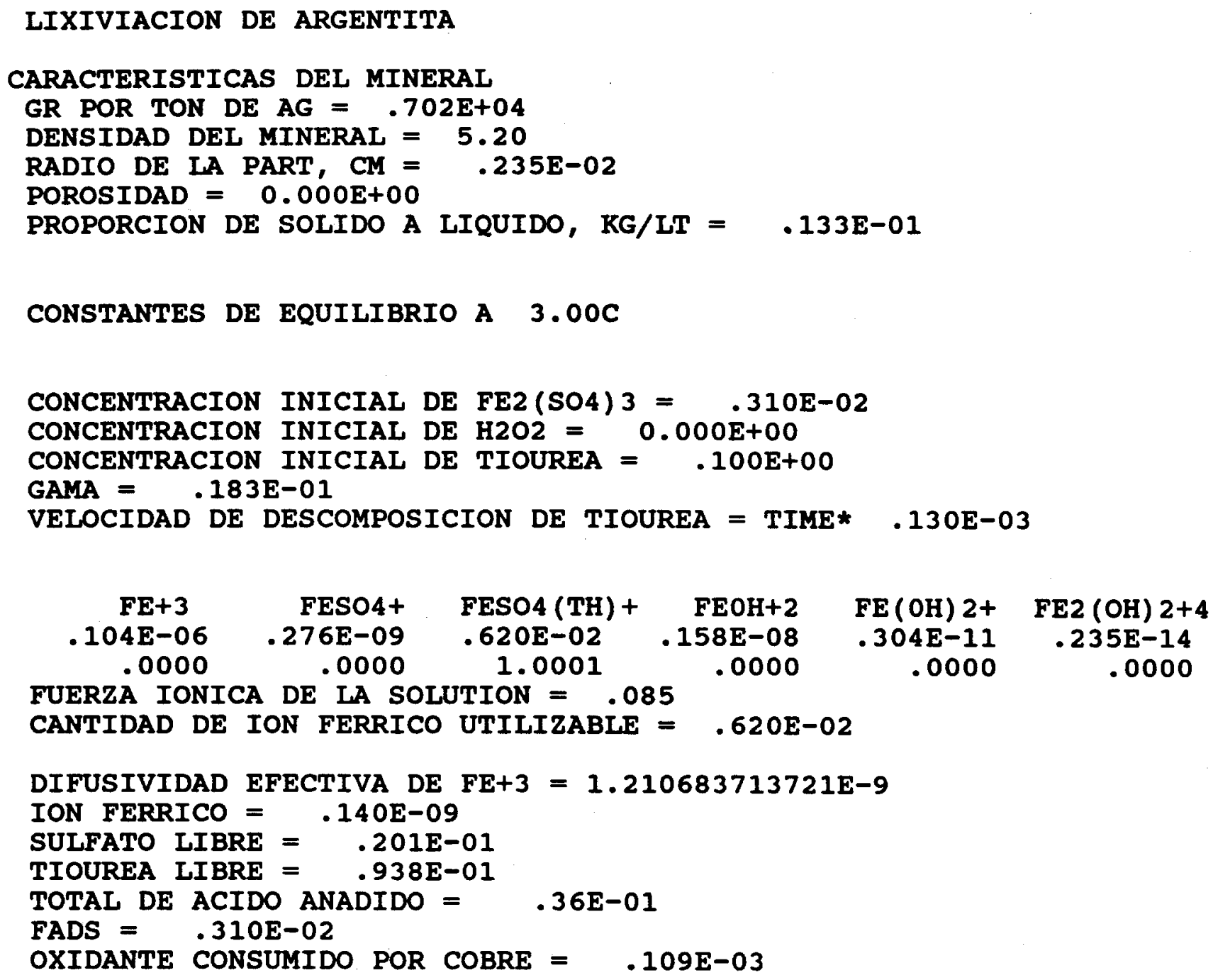

\section{PERFILES ADIMENSIONALES}

TIEMPO DE AJUSTE $=120$.

Ag2T6+2 $=.0001710371231741$ LIMITE $=.000003340169962571$

PRECIPITACION DE Ag2SO4.3Tu.H2O

DIFUSUVIDAD EFECTIVA $=1.026057551928 \mathrm{E}-8$

$\begin{array}{lcccc} & \text { PH } & \mathrm{REAL}=1.443739601588 & & \\ \mathrm{Fe}^{3+} & .519 \mathrm{E}-08 & .887 \mathrm{E}-08 & .189 \mathrm{E}-07 & .226 \mathrm{E}-07 \\ \mathrm{SO}_{4}{ }^{2-} & .341 \mathrm{E}+01 & .337 \mathrm{E}+01 & .328 \mathrm{E}+01 & .325 \mathrm{E}+01 \\ \mathrm{H}^{+} & .919 \mathrm{E}+01 & .921 \mathrm{E}+01 & .926 \mathrm{E}+01 & .928 \mathrm{E}+01 \\ \mathrm{Tu}^{2+} & .136 \mathrm{E}+02 & .139 \mathrm{E}+02 & .148 \mathrm{E}+02 & .151 \mathrm{E}+02 \\ \mathrm{Fe}^{2+} & .280 \mathrm{E}+00 & .280 \mathrm{E}+00 & .280 \mathrm{E}+00 & .280 \mathrm{E}+00 \\ \mathrm{AgTu}_{2}{ }^{+} & .209 \mathrm{E}-02 & .165 \mathrm{E}-02 & .445 \mathrm{E}-03 & .500 \mathrm{E}-05\end{array}$


Apéndice D - Experimento 19

$\begin{array}{llcrrrrrr} & & \text { AGt } & \text { AGT+ } & \text { AGT2+ } & \text { AGT3+ } & \text { AGT4+ } & \text { AG2T3+2 } & \text { AG2T6+2 } \\ \text { EN LA SUPER } & .1 \mathrm{E}-14 & .4 \mathrm{E}-06 & .1 \mathrm{E}-04 & .2 \mathrm{E}-03 & .4 \mathrm{E}-04 & .2 \mathrm{E}-03 & .48 \mathrm{E}-05 \\ \text { EN SOLUCION } & .3 \mathrm{E}-17 & .8 \mathrm{E}-09 & .3 \mathrm{E}-07 & .4 \mathrm{E}-06 & .1 \mathrm{E}-06 & .1 \mathrm{E}-08 & .34 \mathrm{E}-10\end{array}$

$\begin{array}{lccccc}\text { ADIM.TIEMPO } & \text { RC/RP } & \text { TIEMPO, MIN } & \text { CONVERSION } & \text { PH NUEVO } \\ & .002 & .993 \mathrm{E}+00 & .141 \mathrm{E}-01 & .200 \mathrm{E}-01 & 1.44 \\ & .027 & .991 \mathrm{E}+00 & .238 \mathrm{E}+00 & .263 \mathrm{E}-01 & 1.44 \\ \mathrm{Fe}^{3+} & .102 & .987 \mathrm{E}+00 & .911 \mathrm{E}+00 & .381 \mathrm{E}-01 & 1.44 \\ \mathrm{SO}_{4}{ }^{2-} & .215 \mathrm{E}-07 & .217 \mathrm{E}-07 & .220 \mathrm{E}-07 & .222 \mathrm{E}-07 & \\ \mathrm{H}^{+} & .326 \mathrm{E}+01 & .326 \mathrm{E}+01 & .325 \mathrm{E}+01 & .325 \mathrm{E}+01 & \\ \mathrm{Tu}^{2+} & .922 \mathrm{E}+01 & .923 \mathrm{E}+01 & .926 \mathrm{E}+01 & .927 \mathrm{E}+01 & \\ \mathrm{Fe}^{2+} & .152 \mathrm{E}+02 & .152 \mathrm{E}+02 & .152 \mathrm{E}+02 & .152 \mathrm{E}+02 & \\ \mathrm{AgTu}_{2}{ }^{+} & .279 \mathrm{E}+00 & .279 \mathrm{E}+00 & .280 \mathrm{E}+00 & .280 \mathrm{E}+00 & \\ & .187 \mathrm{E}-02 & .161 \mathrm{E}-02 & .758 \mathrm{E}-03 & .357 \mathrm{E}-03 & \end{array}$

$\begin{array}{llcrrrrrr} & & \text { AGt } & \text { AGT+ } & \text { AGT2+ } & \text { AGT3+ } & \text { AGT4+ } & \text { AG2T3+2 } & \text { AG2T6+2 } \\ \text { EN LA SUPER } & .1 \mathrm{E}-14 & .3 \mathrm{E}-06 & .1 \mathrm{E}-04 & .2 \mathrm{E}-03 & .4 \mathrm{E}-04 & .1 \mathrm{E}-03 & .47 \mathrm{E}-05 \\ \text { EN SOLUCION } & .2 \mathrm{E}-15 & .6 \mathrm{E}-07 & .2 \mathrm{E}-05 & .3 \mathrm{E}-04 & .9 \mathrm{E}-05 & .5 \mathrm{E}-05 & .17 \mathrm{E}-06\end{array}$

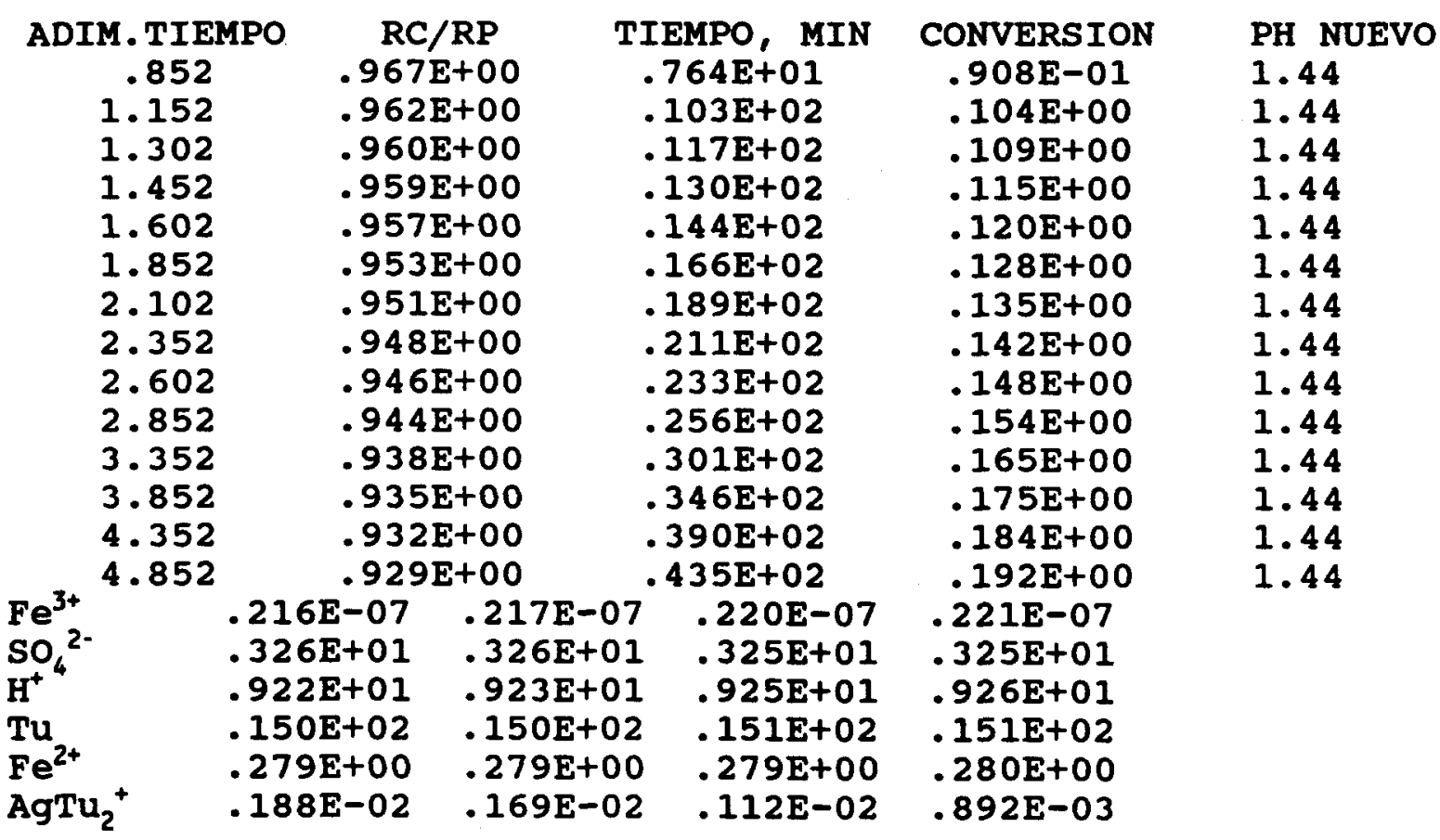

$\begin{array}{llcrrrrrr} & & \text { AGt } & \text { AGT+ } & \text { AGT2+ } & \text { AGT3+ } & \text { AGT4+ } & \text { AG2T3+2 } & \text { AG2T6+2 } \\ \text { EN LA SUPER } & .1 \mathrm{E}-14 & .3 \mathrm{E}-06 & .1 \mathrm{E}-04 & .2 \mathrm{E}-03 & .4 \mathrm{E}-04 & .1 \mathrm{E}-03 & .47 \mathrm{E}-05 \\ \text { EN SOLUCION } & .5 \mathrm{E}-15 & .1 \mathrm{E}-06 & .6 \mathrm{E}-05 & .8 \mathrm{E}-04 & .2 \mathrm{E}-04 & .3 \mathrm{E}-04 & .11 \mathrm{E}-05\end{array}$ 
Apéndice D - Experimento 19

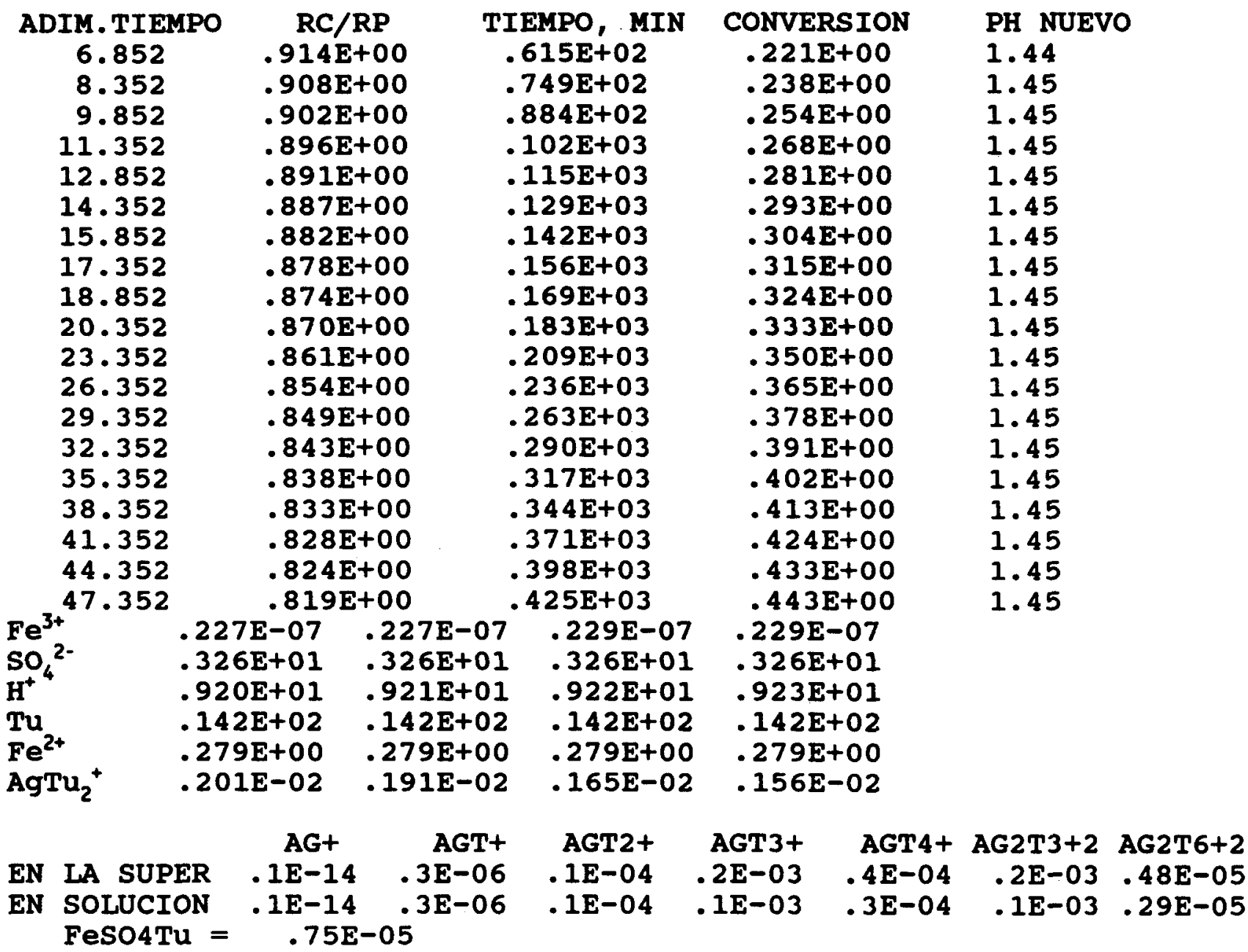

$\begin{array}{ccccl}\text { ADIM.TIEMPO } & \text { RC/RP } & \text { TIEMPO, MIN } & \text { CONVERSION } & \text { PH NUEVO } \\ 55.352 & .806 \mathrm{E}+00 & .497 \mathrm{E}+03 & .465 \mathrm{E}+00 & 1.45 \\ 60.352 & .800 \mathrm{E}+00 & .541 \mathrm{E}+03 & .478 \mathrm{E}+00 & 1.45 \\ 65.352 & .794 \mathrm{E}+00 & .586 \mathrm{E}+03 & .490 \mathrm{E}+00 & 1.45 \\ 70.352 & .788 \mathrm{E}+00 & .631 \mathrm{E}+03 & .502 \mathrm{E}+00 & 1.45 \\ 75.352 & .782 \mathrm{E}+00 & .676 \mathrm{E}+03 & .513 \mathrm{E}+00 & 1.45 \\ 80.352 & .777 \mathrm{E}+00 & .721 \mathrm{E}+03 & .523 \mathrm{E}+00 & 1.45 \\ 85.352 & .771 \mathrm{E}+00 & .766 \mathrm{E}+03 & .533 \mathrm{E}+00 & 1.45 \\ 90.352 & .766 \mathrm{E}+00 & .810 \mathrm{E}+03 & .543 \mathrm{E}+00 & 1.45 \\ 95.352 & .761 \mathrm{E}+00 & .855 \mathrm{E}+03 & .553 \mathrm{E}+00 & 1.45 \\ 100.352 & .755 \mathrm{E}+00 & .900 \mathrm{E}+03 & .562 \mathrm{E}+00 & 1.45 \\ 110.352 & .740 \mathrm{E}+00 & .990 \mathrm{E}+03 & .580 \mathrm{E}+00 & 1.45 \\ 120.352 & .730 \mathrm{E}+00 & .108 \mathrm{E}+04 & .597 \mathrm{E}+00 & 1.45 \\ 130.352 & .719 \mathrm{E}+00 & .117 \mathrm{E}+04 & .614 \mathrm{E}+00 & 1.45\end{array}$

189 


\section{Apéndice D - Experimento 19}

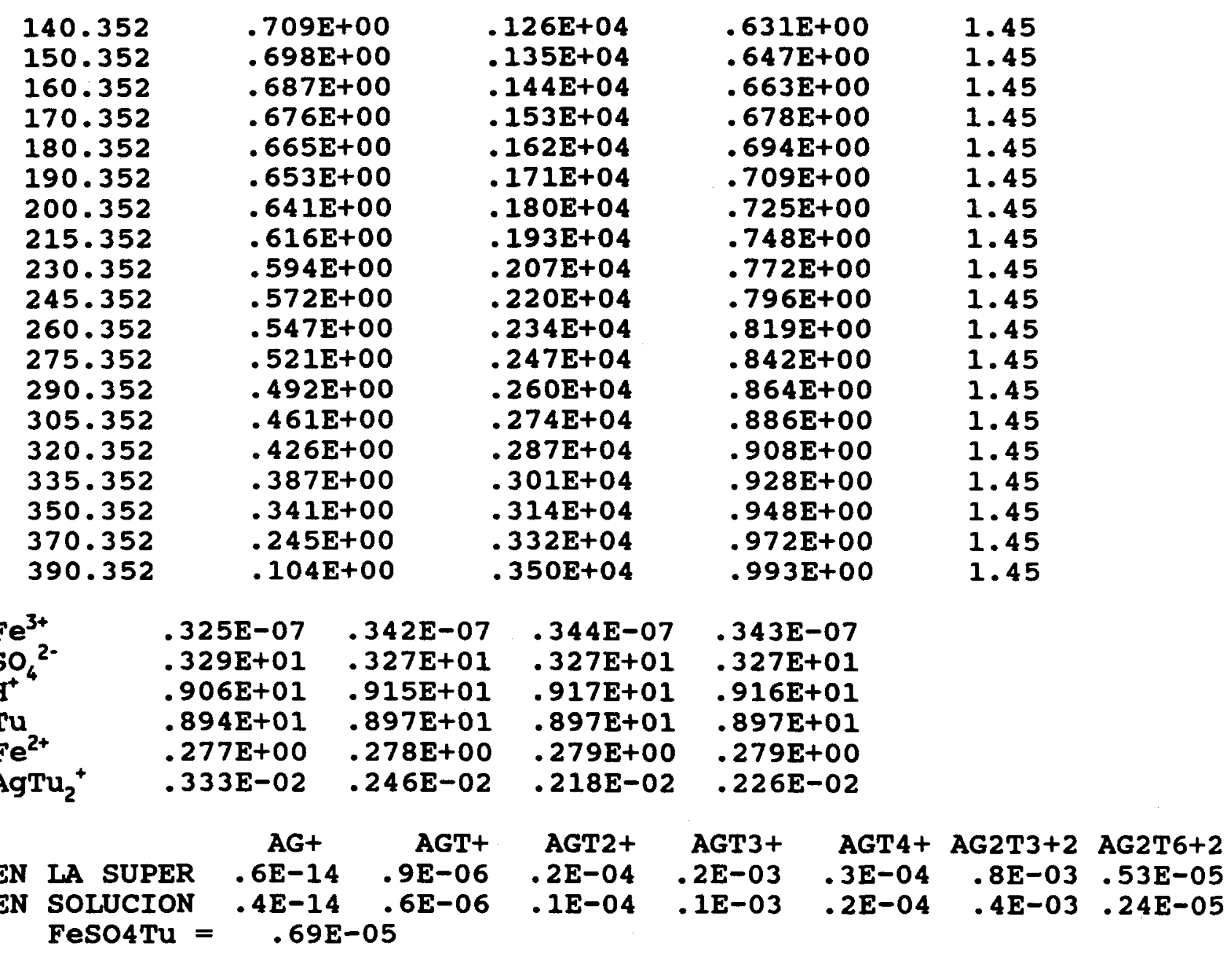

DIFUSIVIDAD EFECTIVA DE FE+3 $=1.026057551928 \mathrm{E}-8$

CONV $=.67 \mathrm{E}-02 * t+-.16 \mathrm{E}-04 * t * * 2+.59 \mathrm{E}-08 * t * * 3$ 
Apéndice D - Experimento 19

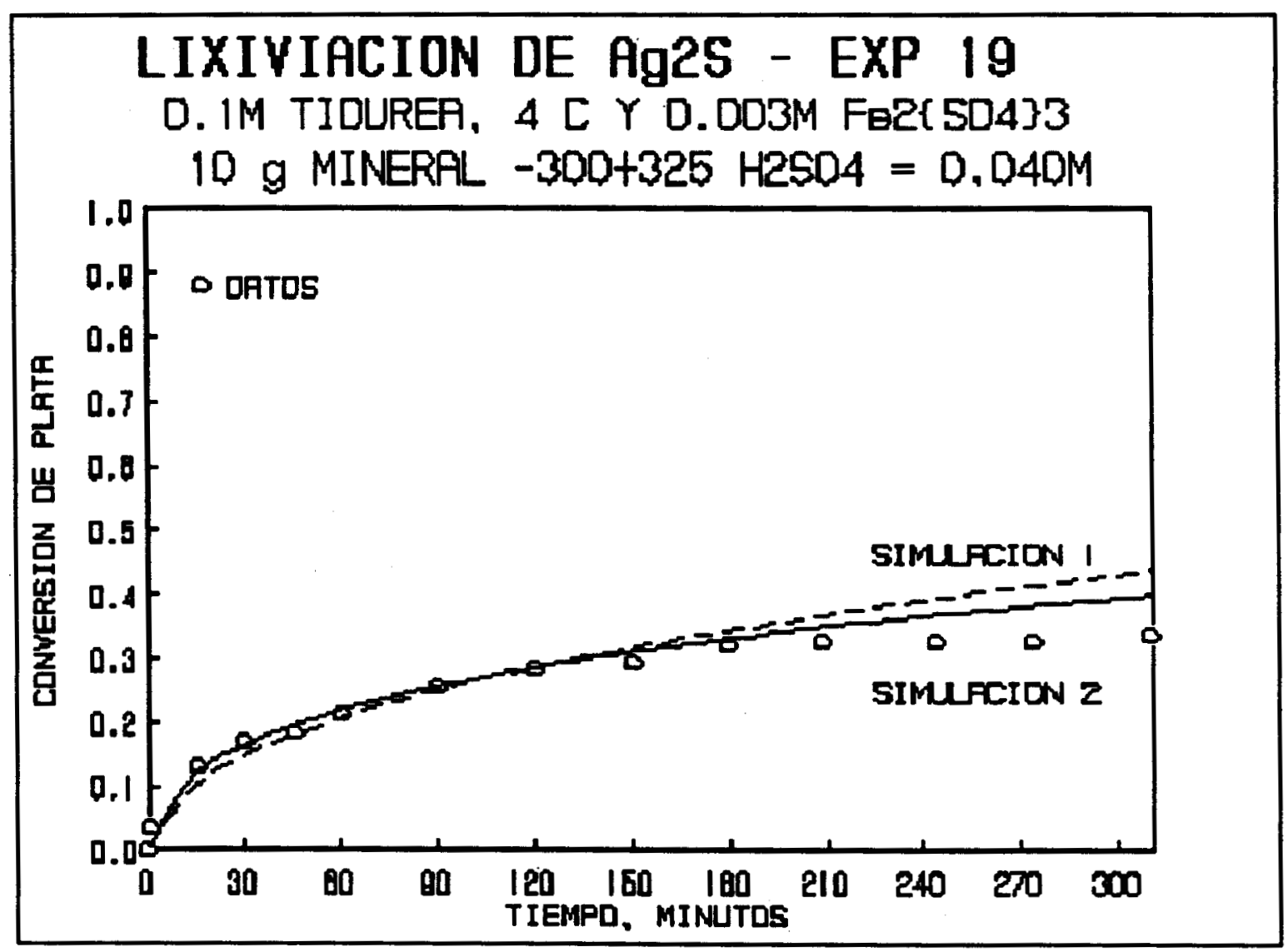

